\title{
Reactor-Specific Spent Fuel Discharge Projections: 1986 to 2020
}

\author{
C. M. Heeb \\ R. C. Walling \\ W. L. Purcell
}

March 1987

Prepared for the U.S. Department of Energy under Contract DE-AC06-76RLO 1830

Pacific Northwest Laboratory Operated for the U.S. Department of Energy by Battelle Memorial Institute 


\title{
DISCLAIMER
}

This report was prepared as an account of work sponsored by an agency of the United States Government. Neither the United States Government nor any agency thereof, nor Battelle Memorial Institute, nor any of their employees, makes any warranty, expressed or implied, or assumes any legal liability or responsibility for the accuracy, completeness, or usefulness of any information, apparatus, product, or process disclosed, or represents that its use would not infringe privately owned rights. Reference herein to any specific commercial product, process, or service by trade name, trademark, manufacturer, or otherwise, does not necessarily constitute or imply its endorsement, recommendation, or favoring by the United States Government of any agency thereof, or Battelle Memorial Institute. The views and opinions of authors expressed herein do not necessarly state or reflect those of the United States Government or any agency thereof, or Battelle Memorial Institute.

\author{
PACIFIC NORTHWEST LABORATORY \\ operated by \\ BATTELLE MEMORIAL INSTITUTE \\ for the \\ UNITED STATES DEPARTMENT OF ENERGY \\ under Contract DE-AC06-76RLO 1830
}

\begin{tabular}{|c|c|}
\hline \multirow{3}{*}{\multicolumn{2}{|c|}{$\begin{array}{l}\text { Printed in the United States of America } \\
\text { Available from } \\
\text { National Technical Information Service }\end{array}$}} \\
\hline & \\
\hline & \\
\hline \multirow{3}{*}{\multicolumn{2}{|c|}{$\begin{array}{c}\text { United States Department of Commerce } \\
5285 \text { Port Royal Road } \\
\text { Springfield, Virginia } 22161\end{array}$}} \\
\hline & \\
\hline & \\
\hline \multicolumn{2}{|c|}{$\begin{array}{l}\text { NTIS Price Codes } \\
\text { Microfiche A01 }\end{array}$} \\
\hline \multicolumn{2}{|c|}{ Printed Copy } \\
\hline & Price \\
\hline Pages & Codes \\
\hline $001-025$ & $\mathrm{~A} 02$ \\
\hline $026-050$ & $\mathrm{~A} 03$ \\
\hline 051-075 & A04 \\
\hline $076-100$ & A05 \\
\hline $101-125$ & A06 \\
\hline $126-150$ & A07 \\
\hline $151-175$ & A08 \\
\hline $176-200$ & A09 \\
\hline $201-225$ & A010 \\
\hline $226-250$ & A011 \\
\hline $251-275$ & A012 \\
\hline $276-300$ & $\mathrm{~A} 013$ \\
\hline
\end{tabular}


REACTOR-SPECIFIC SPENT FUEL

DISCHARGE PROJECTIONS:

1986 TO 2020

C. M. Heeb

R. C. Walling

W. L. Purcell

March 1987

Prepared for

the Office of Civilian Radioactive Waste Management

U.S. Department of Energy

under Contract DE-ACO6-76RLO 1830

Pacific Northwest Laboratory

Richland, Washingt on 99352 

. 


\section{ABSTRACT}

The creation of five reactor-specific spent fuel data bases that contain information on the projected amounts of spent fuel to be discharged from U.S. commercial nuclear reactors through the year 2020 is described. The data bases contain detailed spent-fuel information from existing, planned, and projected pressurized water reactors (PWR) and boiling water reactors (BWR). The projections are based on individual reactor information supplied by the U.S. reactor owners. The basic information is adjusted to conform to Energy Information Agency (EIA) forecasts for nuclear installed capacity, generation, and spent fuel discharged. The EIA cases considered are: 1) No New Orders with Extended Burnup, 2) No New Orders with Constant Burnup, 3) Upper Reference (which assumes extended burnup), 4) Upper Reference with Constant Burnup, and 5) Lower Reference (which assumes extended burnup). Detailed, by-reactor tables are provided for annual discharged amounts of spent fuel, for storage requirements assuming maximum-at-reactor storage, and for storage requirements assuming maximum-at-reactor plus intra-utility transshipment of spent fuel. 

ACKNOWLEDGMENTS

The authors thank K. A. Ekblaw, and R. G. Rau for their assistance in performing the work described in this report. 



\section{CONTENTS}

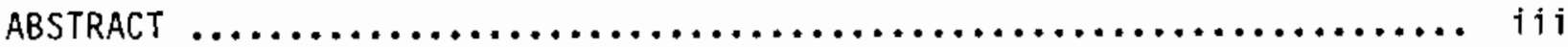

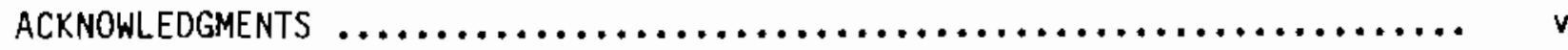

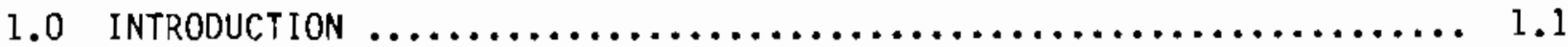

$2.0 \quad$ SUMMARY $\ldots \ldots \ldots \ldots \ldots \ldots \ldots \ldots \ldots \ldots \ldots \ldots \ldots \ldots \ldots \ldots \ldots \ldots, 2.1$

3.0 DISCUSSION $\ldots \ldots \ldots \ldots \ldots \ldots \ldots \ldots \ldots \ldots \ldots \ldots \ldots \ldots \ldots \ldots \ldots \ldots \ldots \ldots \ldots, 3.1$

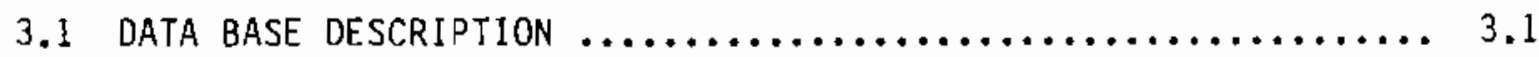

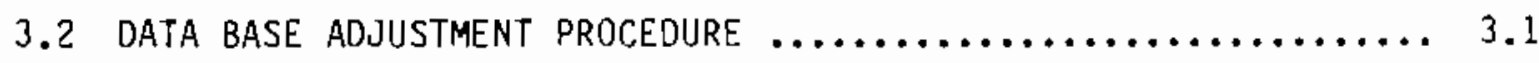

3.2.1 Step 1--Shift Utility Data Base Startup and Shutdown Dates ............................ 3.3

3.2.2 Step 2--Adjustment of Utility Discharges to Match EIA Energy Projections .................. 3.3

3.2.3 Step 3--Adjustment of Burnups to Match EIA Projections $\ldots \ldots \ldots \ldots \ldots \ldots \ldots \ldots \ldots \ldots \ldots \ldots \ldots \ldots . . .3$

3.2.4 Step 4--Calculation of Electrical Energy

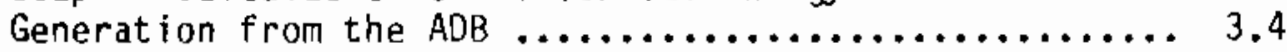

3.3 GENERIC REACTDR ADDITIONS $\ldots \ldots \ldots \ldots \ldots \ldots \ldots \ldots \ldots \ldots \ldots \ldots \ldots \ldots \ldots \ldots$

3.4 ADJUSTED OATA BASE ANNUAL ELECTRIC ENERGY GENERATION $\ldots \ldots \ldots \ldots .3 .9$

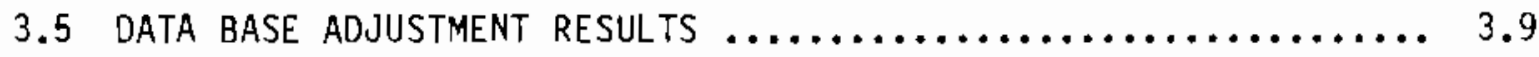

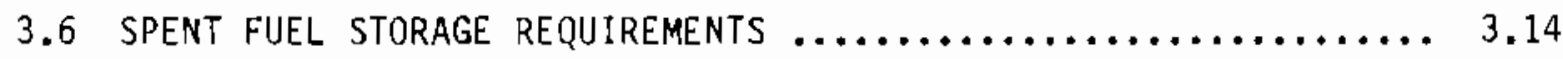

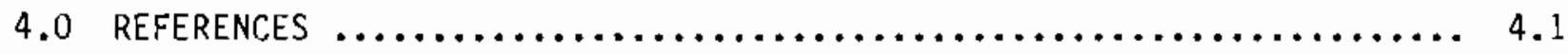

APPENDIX A - DETAILED DATA BASE RESULTS $\ldots \ldots \ldots \ldots \ldots \ldots \ldots \ldots \ldots \ldots \ldots \ldots \ldots \ldots \ldots \ldots \ldots$

APPENDIX B - MAP OF FEDERAL ENERGY REGIONS $\ldots \ldots \ldots \ldots \ldots \ldots \ldots \ldots \ldots \ldots \ldots \ldots \ldots \ldots \ldots \ldots$ 



\section{FIGURES}

3.1 Flow Diagram of the Data Base Adjustment Process for the No New Orders and Reference Cases ....................... 3.2

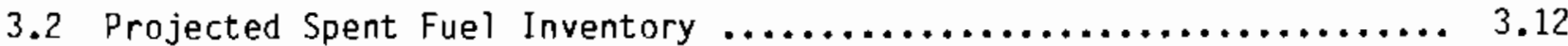

3.3 ElA Installed Nuclear Capacity Projections .................. 3.13

3.4 EIA Nuclear Energy Generation Projection ................... 3.15

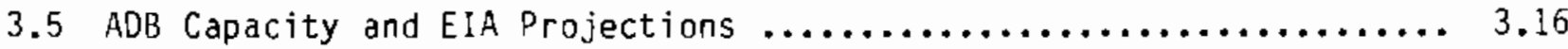

3.6 ADB Energy and EIA Energy Projections $\ldots \ldots \ldots \ldots \ldots \ldots \ldots \ldots \ldots \ldots \ldots \ldots \ldots \ldots$

3.7 Cumulative Additional Storage Requirement--Maximum
At-Reactor Storage $\ldots \ldots \ldots \ldots \ldots \ldots \ldots \ldots \ldots \ldots \ldots \ldots \ldots \ldots \ldots \ldots \ldots \ldots \ldots$

3.8 Cumulative Additional Storage Requirement--Maximum At-Reactor Storage Plus Transshipment $\ldots \ldots \ldots \ldots \ldots \ldots \ldots \ldots \ldots \ldots \ldots .22$

\section{TABLES}

1.1 List of Acronyms for Data Base Names $\ldots \ldots \ldots \ldots \ldots \ldots \ldots \ldots \ldots \ldots \ldots .4$

3.1 Cycle to Batch Energy Ratio by Cycle $\ldots \ldots \ldots \ldots \ldots \ldots \ldots \ldots \ldots \ldots$

3.2 Generic Reactor Properties $\ldots \ldots \ldots \ldots \ldots \ldots \ldots \ldots \ldots \ldots \ldots \ldots \ldots \ldots \ldots \ldots$

3.3 Federal Region Location $\ldots \ldots \ldots \ldots \ldots \ldots \ldots \ldots \ldots \ldots \ldots \ldots \ldots \ldots \ldots$

3.4 Allocation of Generic Reactors to Federal Region $\ldots \ldots \ldots \ldots \ldots . . . . .3$

3.5 Annual Discharges from the Five Adjusted Data Bases (MTIHM) ...... 3.10

3.6 Cumulative Discharges from the Five Adjusted Data Bases (MTIHM) .... 3.11

3.7 Cumulative Storage Requirements--Maximum AR Storage (MTIHM) ...... 3.19

3.8 Cumulative Storage Requirements--Maximum AR Storage Plus

Transshipment (MTIHM) ............................... 3.21 



\subsection{INTRODUCTION}

Under the provisions of the Nuclear Waste Policy Act of 1982 (NWPA), the Department of Energy (DOE) is responsible for the management and ultimate permanent disposal of the civilian radioactive waste generated as a result of commercial nuclear power plant operations in the U.S. The Office of Civilian Radioactive Waste Management (OCRWM) has been established within DOE to plan for and carry out this responsibility.

The greatest portion of the radioactive waste covered under this government responsibility will be spent nuclear fuel discharged from commercial nuclear power plants. Because most of the spent fuel that will ultimately require disposal has not yet been generated, planning for the management and disposal of this spent fuel must be largely based on projections of future spent fuel discharges from commercial nuclear power plants.

The DOE Energy Information Administration (EIA) annually publishes projections of nuclear energy generation on an overall, industry composite basis (DOE/EIA 1986a). In addition, the EIA also publishes projections of spent fuel discharges from commercial nuclear plants which are keyed to each nuclear energy generation projection (DOE/EIA 1986b). These EIA energy projections provide several scenarios representing different assumptions about the future growth of nuclear energy capacity in the U.S. Multiple scenarios allow analysis of the sensitivity of results and decisions to varying assumptions.

The OCRWM plans for management and disposal of spent fuel are based on the EIA nuclear energy projections. However, the EIA projections do not contain the specific reactor-by-reactor information that is needed to perform detailed analyses of relevant issues such as waste system transportation logistics, specific requirements for interim storage of spent fuel, or anticipated variations in the physical characteristics of the spent fuel to be received for disposal. Therefore, to provide a more detailed basis for such analysis, the DOEsponsored work to create adjusted data base sets (ADBS) based on utilitysupplied data, and adjusted to meet EIA projections of annual spent fuel discharges, nuclear capacity, and energy generation. The Pacific Northwest Laboratory (PNL), operated for the DOE by Battelle Memorial Institute, produced 
these ADBs for three time periods. The first ADB set was based on historical data complete through 1983 and projected data from 1984 to 2020 (Heeb, Libby, and Holter 1985). The second set of ADBs included historical information complete through 1984 and projected information from 1985 to 2020 (Heeb et al. 1986). This document describes the ADB set that includes historical data through 1985 collected by EIA in 1986 and projected data for the 1986 to 2020 time period.

In the first study, the basic source of data used to develop the reactorspecific information was the utility supplied Spent Fuel Data Base (SFDB) collected by PNL for the DOE Commercial Spent Fuel Management (CSFM) Program. The second set of ADBs consists of utility data (SFDB) collected by EIA via the RW-859 form and processed by PNL. This set contains historical data through 1984 and projections from 1985 to 2020. The third ADB set described in this report is based on utility information (SFDB) collected by EIA during 1986, and projects for the period from 1986 through 2020. Pacific Northwest Laboratory produced the new ADB set hy adjusting the ElA data from the RW-859 form to meet EIA projections of nuclear capacity, energy generation, and spent-fuel discharges.

The Reactor Data Analysis (RDA) Program uses the adjusted data bases for a number of planning purposes. Roy $F$. Weston Inc. uses adjusted data base information to calculate total system life cycle costs (TSLCC) for OCRWM. The data base also provides the basic information on spent fuel for inclusion in the DOE Integrated Data Base (IDB) maintained by Oak Ridge National Laboratory. It is used in the DOE Nuclear Waste Systems Integration (NWSI) Program as well as the Monitored Retrievable Storage (MRS) Program.

Three 1986 EIA nuclear growth scenarios were chosen as bases for deve1oping the 1986 reactor-specific spent fuel discharge projections: the Upper Reference Case, the Lower Reference Case, and the No New Orders Case. The background assumptions for these cases are described in documents published by DOE (DOE/EIA 1986a and DOE/EIA 1986b). The Upper Reference Case has been previously selected by the OCRWM as the base case for waste management planning purposes. The Lower Reference and the No New Orders Cases were included to provide additional data sets that could be used in analysis of the sensitivity 
of analytical results and management decisions to possible reductions in nuclear growth in the U.S. The projected installed nuclear generating capacity projections for the three growth scenarios are shown in figure 3.2. The projected nuclear electric energy generation projections are shown in Figure 3.3.

The No New Orders Case includes only nuclear power plants that are currently operating and a subset of the plants currently under construction. EIA assumes that some of the plants currently under construction will not be completed, and that the startup of others will be delayed beyond official utility startup estimates. The Upper and Lower Reference Cases include additional power plants, not currently on order, coming on line starting in 2001 and 2006 respectively to provide sufficient power generation capacity to correspond to the overall nuclear energy production rates in the EIA projections. In the No New Orders Case it is assumed that some plants in the construction pipeline will be canceled and the startup dates of pipeline plants will be delayed when compared with the Upper Reference Case. The Lower Reference Case contains the same set of plants under construction (pipeline plants) as the No New Orders Case, however a modest addition of generic plants is assumed after 2005.

In making projections of the amount of spent fuel discharged, EIA considers cases of constant and extended burnup. In the Upper and Lower Reference Cases it is assumed by 1998 that the reactors will extend their current burnups by 30 percent. The discharge burnups for these cases are then assumed constant beyond 1998.

An Upper Reference Case with constant burnup was also considered. In this case, the burnup level was held at the 1979 to 1983 historical levels through 2020. Thus, at projected annual energy generations forecasted by the Upper Reference Case, two spent-fuel discharge projections are made corresponding to the constant and extended burnup assumptions (DOE/EIA 1986b, Table E.2). A similar set of assumptions for burnup are made for the energy generation forecasted in the No New Orders Case.

In order to meet the installed capacity and energy-generation requirements beyond the year 2000 for the Reference Cases, it was necessary to add generic power plants to the population of operating and pipeline reactors. Reactor types consistent with currently existing power plants were chosen to represent 
these generic power plants. This permitted the detafled information for the generic power plants to be obtained from the ADB in the same manner as for the currently existing or planned reactors. Each of the generic reactors was assigned to a specific federal energy region. A mixture of two thirds pressur.ized water reactors (PWRs) and one third bofling water reactors (BWRs) was assumed to conform with EIA Reference Case Assumptions.

The data-base names are abbreviated as shown in Table 1.1.

TABLE 1.1. List of Acronyms for Data Base Names

\begin{tabular}{ll} 
Acronym & \multicolumn{1}{c}{ Data Base } \\
\cline { 3 - 3 } SFDB & $\begin{array}{l}\text { The Unmodified Spent Fuel Data Base Containing Only Utility } \\
\text { Supplied Data. This data is used for the spent Fuel Requir } \\
\text { ments Report (DOE/RL 1986) and for many other purposes. }\end{array}$ \\
ADB & Any Adjusted Data Base \\
NNE & No New Orders Case, Assumes Extended Burnup \\
NNC & No New Orders Case Sensitivity Case with Constant Burnup \\
URE & Upper Reference Case, Assumes Extended Burnup \\
URC & Upper Reference Case Sensitivity Case with Constant Burnup \\
LRE & Lower Reference Case, Assumes Extended Burnup
\end{tabular}

The adjusted data base names are keyed to case names published in World Nuclear Fuel Cycle Requirements, 1986 (DOE/EIA 1986b, Tables E.1 and E.2). 


\subsection{SUMMARY}

The utility-supplied spent-fuel discharge forecasts contained in the 1986 SFDB were adjusted to produce agreement with EIA discharge forecasts (DOE/EIA 1986b). Adjustments were made also to utility-supplied projected discharge burnups in the 1986 SFDB to produce agreement with EIA burnup and energygeneration assumptions (DOE/EIA 1986a). Five reactor-specific ADBs were produced to match five EIA cases. The ADBs are designated as follows:

1. No New Orders, Extended Burnup-NNE

2. No New Orders, Constant Burnup-NNC

3. Upper Reference, Extended Burnup-URE

4. Upper Reference, Constant Burnup-URC

5. Lower Reference, Extended Burnup-LRE.

The adjustment procedure changes the amount of fuel discharged annually so that it agrees with the EIA forecast. The method allows the discharged fuel batch identity of the utility data base to remain intact, while exact agreement with EIA projected spent fuel discharges is obtained by adjusting batch size. Burnup is then adjusted to produce agreement with EIA burnup and energy generation forecasts.

The method used in developing the reactor-specific spent fuel discharge projections, as well as the resulting data bases themselves, are described in detail in this report. Discussions of the method cover the following topics:

- description of the data base

- data base adjustment procedures

- addition of generic power reactors

- calculated data base annual electric energy generation.

The accumulated spent fuel inventory for each of the five cases analyzed, and the original utility inventory projection are presented in Chapter 3.0 .

Reactor-specific discharge and storage requirements for four scenarios are presented in Appendix A. Table A.1 gives startup and shutdown dates for reactors in the four scenarios: the Utility case, the No New Orders Case, the Upper Reference Case, and the Lower Reference Case. Table A.2 gives the names and startup dates for the Upper and Lower Reference Case generic reactors. 
Tables A.3 and A.4 show the Upper Reference Case (URE) annual and cumulative discharges respectively. Tables A.5 through A.8 display the annual and cumulative storage requirements for the maximum at-reactor (AR) storage assumption, and for the maximum transshipment assumption for the URE data base. These compare directly to the storage requirements from the utility-supplied data, as reported by the DOE Richland Operations Office (DOE/RL 1986).

The reactor-specific spent fuel discharge projections contained in this report cover the period from 1986 through 2020. This covers a total cumulative spent fuel inventory of 123,667 MTU for the Upper Reference Case, Constant Burnup to 79,550 MTU for the No New Orders, Extended Burnup Case. A description of the methods and the results is included in the next section of this report. Detailed information that supports discussions in the main body of the report, including descriptions of the capacity and fuel discharge projections, are included in Appendix A. 


\section{- $\quad 3.0$ DISCUSSION}

The 1986 SFDB is a compendium of information on U.S. cormercial power reactor spent fuel and other reactor-specific information collected by EIA via the RW-B59 form. It is based on data provided by the operating utility, and represents their estimate of the amounts and characteristics of the spent-fuei discharges. Electrical energy generation is not contained explicitly in the data base, but it may be derived from the spent-fuel quantities and spent-fue 1 burnup contained in the data base.

\subsection{DATA BASE DESCRIPTION}

The SFDB contains a file for each reactor. The first portion of the file consists of time-independent information such as the location, various power ratings, dates of start-up and final shutdown, and detailed information on fuel stored in the reactor's pool(s). The second portion consists of the historical record of fuel discharges by batch or sub-batch with discharge exposure range, number of assemblies, and the uranium mass for each batch. The succeeding portions of the file are organized by operating cycle, that is, by the period between successive discharges. The third portion of the SFDB contains projected discharge information for 1986 and beyond.

The electrical energy generation implicit in the SFDB is entirely independent of the nuclear energy generation forecasts made by EIA. The SFDB was modified to produce an $A D B$ that is consistent with these nuclear-energy generation and discharged-fuel amount forecasts. The technical steps involved in modifying the SFDB to produce an ADB that conforms to the EIA projections are described in this section. The principal requirement of the modification was to retain as much of the detailed utility estimates of exposure, plant capacity factor, and discharge schedules as possible, while matching the EIA spent-fuel discharged amount, and energy-generation forecasts.

\subsection{DATA BASE ADJUSTMENT PROCEDURE}

The steps in the adjustment procedure are shown in Figure 3.1. The adjustment process required the construction of four major processors. Each of 
1

Legend: $\square$ Processing Step $\square$ Data Base

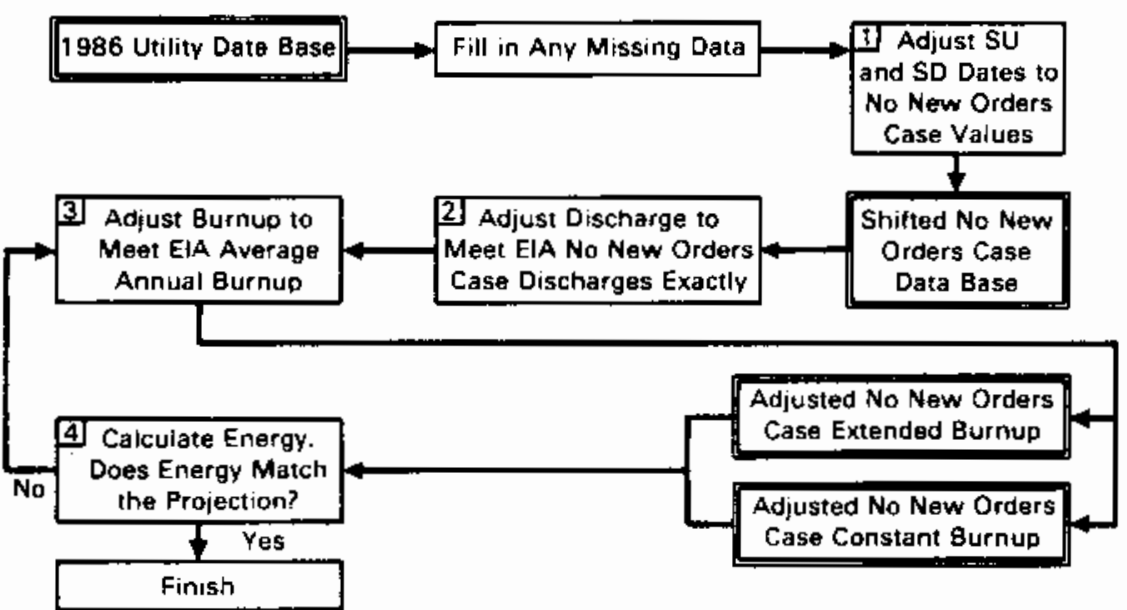

Data Base Adjustment Process for the No New Orders Case

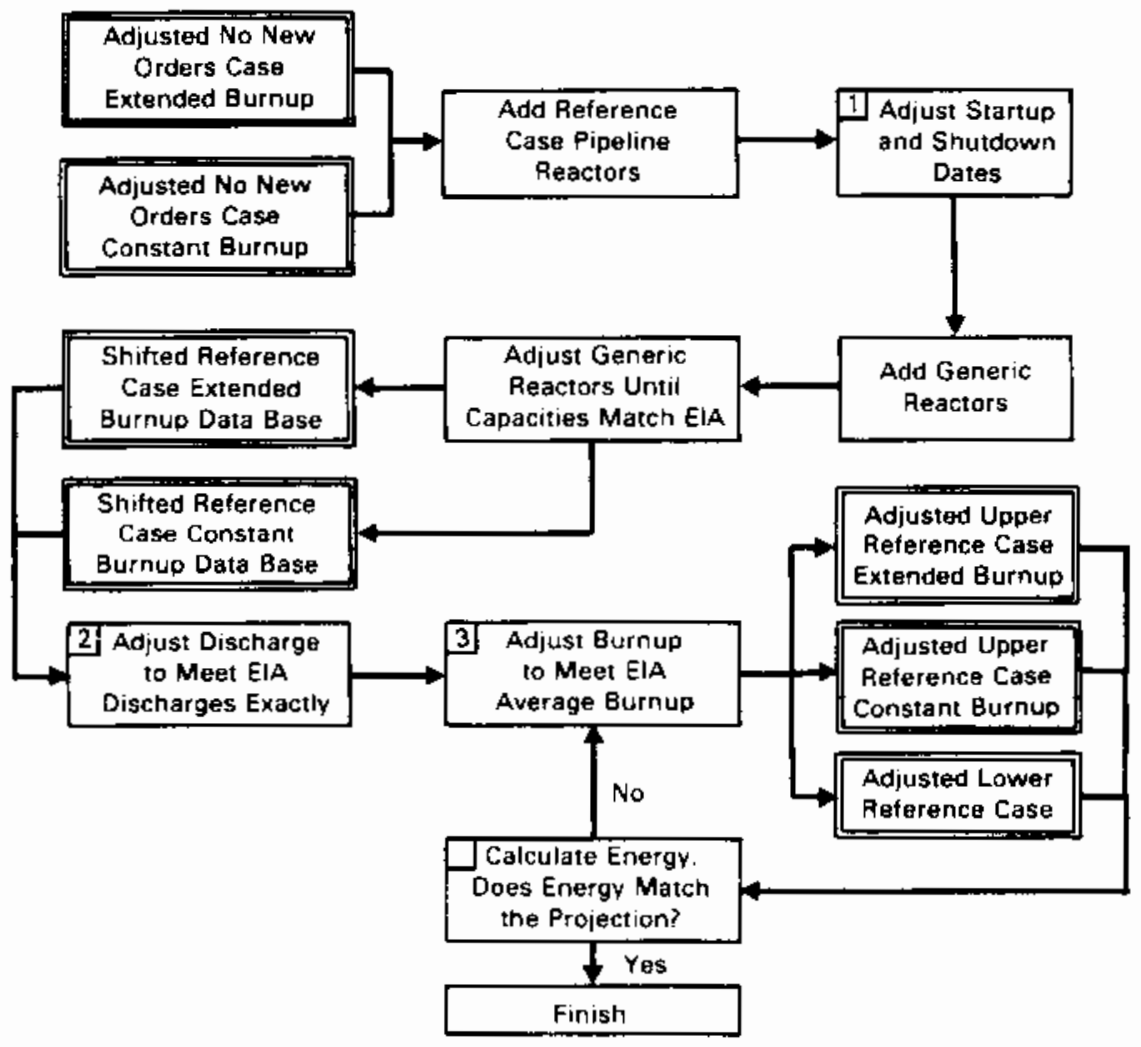

Data Base Adjustment Process for the Reference Cases

FIGURE 3.1. Flow Diagram of the Data Base Adjustment Process for the No New Orders and Reference Cases 
these is described in the sections that follow. The adjustment process is entirely concerned with the time beyond 1985, since historical information is left unchanged by the adjustment process. The adjustment steps are numbered in the Figure 3.1 flow chart.

\subsubsection{Step 1--Shift Utility Data Base Startup and Shutdown Dates}

EIA operable dates published by DOE/EIA (1986a) for pipeline reactors starting up from 1986 to 2020 were shifted six months later to represent the startup date for commercial operation, which is the starting date of energy generation used by the $A D B$ energy calculation algorithim. The start-up date for each reactor is shown in Appendix A, Table A.1. Pipeline reactors that are assumed by EIA to be canceled are removed.

The utility-suppiied pattern of discharges was also shifted six months later than the EIA operable date. The shutdown dates were adjusted to 40 years after the adjusted startup date. Generic reactors were added to the Reference Cases so that installed nuclear generation capacity could be matched. The installed capacity match was accomplished at the end of Step 1.

\subsubsection{Step 2--Adjustment of Utility Discharges to Match ElA Energy Projections}

The projected discharge amounts in the SFOB are modified to agree with EIA data published by OOE/EIA (1986b). An adjustment ratio, which is equal to the ratio of the EIA target amount to the unadjusted amount from the SFDB, was calculated for each year. In producing the No New Orders ADBs, all discharges were adjusted by this ratio. In producing the Reference Case ADBs, the adjustment was applied only to reactors starting up after 1985 . In these cases, a larger adjustment factor was required to match the EIA target discharge because not all reactors were eligible for adjustment. The No New Orders ADB discharges from reactors operating before 1986 were therefore preserved as a subset of the Reference Case data bases.

\subsubsection{Step 3--Adjustment of Burnups to Match EIA Projections}

In Step 3 the average annual burnup was adjusted to meet EIA average burnup forecasts. A limit of 60,000 MWD/MTIHM was placed on individual PWR burnups, and a limit of 50,000 MWD/MTIHM was used as the maximum BWR burnup. 
EIA does not publish actual average annual burnups of spent-fuel discharge projections, hence utility-supplied burnups were increased according to EIA's stated increased burnup assumptions ( $30 \%$ by 1998) in the extended burnup cases. Some slight changes were made to the average annual burnups of all adjusted data bases in order to match EIA annual energy generation forecasts.

\subsubsection{Step 4--Calculation of Electrical Energy Generation from the ADB}

The information available on the data base permits an estimation of the electrical energy generated by the fuel during its residence in the core. Energy generation in a batch of discharged fuel is equal to the product of the burnup (MWD/MTIHM) and the amount discharged (MTIHM). This is the therma? energy generated in the fuel. The electrical energy is the product of the thermal energy and the thermal efficiency of the reactor. In order to caiculate the annual electrical generation by the reactor it is necessary to relate the thermal energy generated by the fuel to the electrical energy generated by the reactor. The algorithm employed to do this is based on the relative energy generated by the fuel batches during equilibrium operation. The algorithm is then modified to include non-equilibrium initial core batches.

During equilibrium operation, each position in the core is designated as one that holds fresh fuel, once-burned fuel, twice burned fuel, etc. The batch of fresh fuel going in will generate $E_{1}$ kWh during its first cycle of residence. During the next cycle, the batch of fuel will be moved into the designated once-burned fuel positions in-core and generate $E_{2} \mathrm{kWh}$. This progression continues until the discharge cycle is reached. During this cycle let $E_{N}$ be the final energy generated by the batch during the last cycle of residence. The energy generated by the discharged batch, $E_{D}$, will be the sum of the energy generated in each cycle of residence, $E_{D}=E_{1}+E_{2}+\ldots \ldots E_{N}$.

During any give cycle of operation, however, there will be a batch of fresh fuel in-core that witl generate $E_{1} \mathrm{kWh}$ and a once-burned batch in-core that will generate $E_{2} \mathrm{kWh}$. This progression will continue until the highestburned batch is reached, which will generate $E_{N} k W h$. The energy generated by the reactor during any cycle of equilibrium operation, $E_{R}$, will be the sum of the energy generated by all fuel batches resident in-core during the cycle, $E_{R}=E_{1}+E_{2}+\ldots . E_{N}$. Hence, $E_{D}$ equals $E_{R}$. This key relationship is used to 
calculate the cycle energy from the amount discharged and the burnup, parameters that are readily available on the data base.

For reactors just starting up, the ratio of first-cycle energy generation to first-discharge batch energy generation will not be unity, but will approximate the reciprocal of the core fraction discharged if power sharing by in-core batches is proportional to batch size. Thus for one-third core replacement, the ratio of first cycle energy to the energy generated by the fuel in the first discharge would approximate 3.0. The second discharge ratio would approximate half of this, or 1.5. The third discharge ratio would approximate unity, and remain unity for all subsequent discharges.

In actual practice power sharing between in-core fuel batches is not exactly equitable, and fuel management plans do not specify that the same fraction of the core will be replaced for every refueling outage. However, when large numbers of reactors are involved and interest is primarily in over-all energy generation over several years during which only a small fraction of the total energy generation is from plants not at equilibrium, average nonequilibrium ratios of cycle energy to batch energy may be approximated with sufficient accuracy. Detailed fuel management plans for several reactors were andyzed to obtain a more realistic ratio of cycle to batch energy. The ratios of cycle energy to batch energy $\left(E_{R} / E_{D}\right)$ were calculated and the results are summarized in Table 3.1 .

TABLE 3.1. Cycle to Batch Energy Ratio by Cycle

\begin{tabular}{|c|c|c|c|c|c|}
\hline & First & Second & Third & Fourth & Equilibrium \\
\hline PWR & 2.857 & 1.266 & 1.095 & 1.073 & 1.000 \\
\hline$B W R$ & 3.663 & 1.404 & 1.111 & 1.058 & 1.000 \\
\hline
\end{tabular}

The energy of a given cycle is approximated by adding the product of total burnup, weight of the batch, and the thermal efficiency of the reactor over all batches discharged at the end of the cycle. This energy is $E_{D}$, the energy generated by the discharged fuel. If the reactor is at equilibrium, 
then $E_{D}$ equals $E_{R}$, as has been demonstrated; the energy generated in the discharged fuel is the energy generated by the reactor during the operating cycle. If the reactor is not at equilibrium, then the appropriate factor from Table 3.1 is multiplied by $E_{D}$ to obtain $E_{R}$, the cycle energy. For example, if the reactor were a PWR, and was its second cycle of operation, then

ER equals $1.266 \times E_{D}$.

The annual energy generation is calculated from $E_{R}$ by allocation to each year according to the number of months in the year for that cycle. Thus if a 17 month cycle had one month in year $x, 12$ months in year $(x+1)$, and the remaining four months in year $(x+2)$, then the reactor would contribute to each of the three year's energy generation as follows: year $x$ would contribute $1 / 17 \times E_{R}$; year $(X+1)$ would contribute $12 / 17 \times E_{R}$; and year $(X+2)$ would contribute $4 / 17 \times E_{R}$. The annual contributions from each reactor cycle calculated in this manner are added together to compute the annual energy generation schedule for comparison to EIA energy generation forecasts.

\subsection{GENERIC REACTOR ADDITIONS}

The SFDB contains information only on those reactors that were operating, under construction, or in the planning stage by some U.S. utility in 1985 . In order to meet EIA annual spent-fuel discharged amounts, installed nuclear capacity, and energy-generation forecasts after 2000 for the Reference Cases, it is necessary to include generic reactors in the shifted data base after Step 1 in the adjustment process (see previous discussion and Figure 3.1 ).

Two actual reactors were selected to represent the generic PWR and BWR. Both were nominal 1100 Mwe plants and both were on an average 18 month refueling schedule. The PWR design exposure was 37,800 MWD/MTIHM, and the BWR burnup was 36,000 MWD/MTIHM. Relevant details for the two generic plant types are shown in Table 3.2 .

Increases in capacity do not in general represent the addition of an integral number of plants, each with a fixed plant capacity. Fractional additions to capacity were represented by delayed startup of one of the plants of each plant type that are otherwise started up in January each year to maintain the correct cumulative capacity. Thus if the accumulated new capacity was 
TABLE 3.2. Generic Reactor Properties

เ

\begin{tabular}{|c|c|c|}
\hline & PWR & $B W R$ \\
\hline Rated Power Level & $1,100 \mathrm{MWe}$ & $1,100 \mathrm{MWe}$ \\
\hline Thermal Power Level & $3,411 \mathrm{Mwth}$ & 3,320 Mwth \\
\hline Average Fuel Cycle Length & 18 Months & 18 Months \\
\hline Equilibrium Enrichment & $3.70 w t \%$ & $3.0 \mathrm{wt} \%$ \\
\hline Discharge Exposure & $37,800 \mathrm{MWD} / \mathrm{MTIHM}$ & $36,000 \mathrm{MWD} / \mathrm{MTIHM}$ \\
\hline
\end{tabular}

equivalent to 15.65 generic plants by a given year, one of the plants would be started up in the fourth month of that year: $(1.0-0.65) \times 12.0=4.2$. The start-up of integral additions to capacity were done in accordance with the EIA convention of commercial operation additions in July of each year, with a six month interval between first eiectrical generation and commercial operation.

It is important for logistics modeling to maintain reasonable geographic accuracy in the projected spent-fuel discharges. This requires that the generic reactors be added with site diversity. The first step in providing site diversity is to assign each generic reactor to a federal energy region. The location of each federal energy region is shown in Table 3.3, and a map of the regions is reproduced in Appendix $B$.

TABLE 3.3. Federal Region Location

\begin{tabular}{rll} 
Federal Region & & \multicolumn{1}{c}{ Location } \\
II & & New England \\
III & & Middle Atlantic \\
IV & South Atlantic \\
$V$ & Midwest \\
VI & Southwest \\
VII & Central \\
VIII & North Central \\
IX & West \\
$X$ & Northwest
\end{tabular}


Site diversity is assured by making the assumption that the regional capacity distribution exhibited by the No New Orders Case in 1995 will continue until 2020. This projects the regional installed capacity distribution for the succeeding 25 year period without change. This assumption was implemented by apportioning the addition of generic reactors to each region according to the 1995 No New Orders distribution. Table 3.4 shows the 1995 No New Orders Case installed capacities and

TABLE 3.4. Allocation of Generic Reactors to Federal Region

\begin{tabular}{|c|c|c|c|c|c|c|}
\hline \multirow{3}{*}{$\begin{array}{l}\text { Federal } \\
\text { Energy } \\
\text { Region } \\
\end{array}$} & \multirow{2}{*}{\multicolumn{2}{|c|}{$\begin{array}{l}\text { NNO } 1995 \\
\text { Capacity }\end{array}$}} & \multicolumn{4}{|c|}{ Generic Reactors } \\
\hline & & & \multicolumn{2}{|c|}{$\begin{array}{c}\text { Upper } \\
\text { Reference }\end{array}$} & \multicolumn{2}{|c|}{$\begin{array}{l}\text { Lower } \\
\text { Reference }\end{array}$} \\
\hline & (MWe) & $(\%)$ & No. & $(\%)$ & No. & (\%) \\
\hline I & 6,509 & 6.2 & 9 & 6.4 & 4 & 6.5 \\
\hline II & 8,707 & 8.3 & 12 & 8.6 & 5 & 8.1 \\
\hline III & 12,676 & 12.0 & 17 & 12.1 & 8 & 12.9 \\
\hline IV & 32,296 & 30.7 & 42 & 30.0 & 19 & 30.6 \\
\hline V & 21,789 & 20.7 & 29 & 20.7 & 13 & 21.0 \\
\hline$V I$ & 7,284 & 6.9 & 10 & 7.1 & 4 & 6.5 \\
\hline VII & 3,234 & 3.1 & 4 & 2.9 & 2 & 3.2 \\
\hline VIII & 330 & 0.3 & 0 & 0.0 & 0 & 0.0 \\
\hline IX & 10,199 & 9.7 & 14 & 10.0 & 6 & 9.7 \\
\hline$x$ & 2,175 & 2.1 & 3 & 2.1 & 1 & 1.6 \\
\hline Tota?s & 105,199 & 100.0 & 140 & 100.0 & 62 & 100.0 \\
\hline
\end{tabular}

the number of Reference Case generic reactors in each region. Since each generic reactor is rated at 1100 MWe, the capacities are directly proportional to number of generic reactors. Hence the comparison of No New Orders Case percentage capacity and the percentage of the number of generic reactors in Table 3.4 is reasonable. Table A.2 in Appendix A shows the actual federal energy region placement and date of commercial operation for each generic reactor. The siting of generic plants within a given federal region was accomplished using the method described in Holter et. al. (1986). The method develops a numerical rating for each postulated generic site in each of the 10 federat energy regions. The numerical rating defines the sequence in which 
each potential site is chosen within a specific region. The generic reactors are then assigned chronologically in order of their startup date using this priority.

To match the EIA projections of installed nuclear capacity for the Upper Reference Case, 93 generic PWRs and 47 generic BWRs were required (a total of 140 generic reactors or a 154 GWe generic reactor capacity). For the Lower Reference Case, 41 generic PWRs and 21 generic BWRs were necessary. The 62 Lower Reference Case generic reactors represent a 68.2 GWe generic reactor capacity.

\subsection{ADJUSTED DATA BASE ANNUAL ELECTRIC ENERGY GENERATION}

The data base annual energy generation calculational procedure is not an exact process. Approximations are required because the data base contains no information on electrical generation. The calculation of annual energies from operating cycle energies as described in Section 3.2.4 reflects the altered discharge amounts and the burnup assumptions imposed on the utility data. The method of calculation: the estimation of annual generation from the product of amount discharged and burnup, will necessarily cause year-to-year fluctuations in the apparent annual energy generation. The level of approximate agreement is a measure of the consistency of EIA projections of spent-fuel discharges, burnup, annual electric energy generation, and detailed utility data.

\subsection{DATA BASE ADJUSTMENT RESULTS}

The projected annul spent fuel discharged amounts for all five ADBs are shown in Table 3.5. These amounts agree, within the number of significant figures given in the EIA projections, with the estimates published by DOE/EIA (1986b, Table E1).

The cumulative discharged amounts are shown in Table 3.6 for all five adjusted data bases. These data should be compared with the data published by DOE (DOE/EIA 1986b, Table E2). In Table E2 of the DOE/EIA Report, the inventory of spent fuel in 1985 was based on a preliminary estimate of 12,400 MTIHM. The final inventory is 12,651 MTIHM, which is the amount used in the Spent Fuel Storage Requirements report (DOE/RL 1986). When this starting difference of 
251 MTIHM is taken into account the inventory schedule in Table 3.6 agrees with EIA Table E2 within the number of significant figures shown in the EIA projection. The spent fuel inventory projections are shown in Figure 3.2.

TABLE 3.5. Annual Discharges from the Five Adjusted Data Bases (MTIHM)

\begin{tabular}{|c|c|c|c|c|c|}
\hline \multirow[b]{2}{*}{ Year } & \multicolumn{2}{|c|}{ No New Drders } & \multicolumn{2}{|c|}{ Upper Reference } & \multirow{2}{*}{$\begin{array}{c}\text { Lower } \\
\text { Reference }\end{array}$} \\
\hline & Ext. Burnup & Const. Burnup & Ext. Burnup & Const. Burnup & \\
\hline 1985 & 1200 & 1200 & 1200 & 1200 & 1200 \\
\hline 1986 & 1400 & 1400 & 1400 & 1400 & 1400 \\
\hline 1987 & 1500 & 1500 & 1548 & 1592 & 1500 \\
\hline 1988 & 1700 & 1700 & 1840 & 1800 & 1700 \\
\hline 1989 & 1900 & 1900 & 2100 & 2100 & 1900 \\
\hline 1990 & 2000 & 2000 & 2000 & 2200 & 2000 \\
\hline 1991 & 2100 & 2100 & 2000 & 2200 & 2100 \\
\hline 1992 & 2100 & 2200 & 2193 & 2299 & 2100 \\
\hline 1993 & 2100 & 2100 & 2000 & 2200 & 2100 \\
\hline 1994 & 2000 & 2100 & 2000 & 2207 & 2000 \\
\hline 1995 & 1999 & 2300 & 2100 & 2400 & 1999 \\
\hline 1996 & 2000 & 2199 & 2000 & 2500 & 2000 \\
\hline 1997 & 1800 & 2100 & 2000 & 2400 & 1800 \\
\hline 1998 & 1900 & 2300 & 1991 & 2499 & 1900 \\
\hline 1999 & 2000 & 2300 & 2000 & 2500 & 2000 \\
\hline 2000 & 1900 & 2200 & 2100 & 2500 & 1900 \\
\hline 2001 & 1900 & 2300 & 2000 & 2500 & 1900 \\
\hline 2002 & 1899 & 2300 & 2000 & 2509 & 1899 \\
\hline 2003 & 2001 & 2300 & 2200 & 2700 & 2001 \\
\hline 2004 & 2019 & 2323 & 2319 & 2824 & 2000 \\
\hline 2005 & 1880 & 2277 & 2280 & 2776 & 1899 \\
\hline 2006 & 2000 & 2299 & 2600 & 3100 & 2000 \\
\hline 2007 & 2100 & 2400 & 2700 & 3399 & 2100 \\
\hline 2008 & 1900 & 2400 & 2599 & 3400 & 1900 \\
\hline 2009 & 2100 & 2500 & 3100 & 3600 & 2199 \\
\hline 2010 & 2300 & 2600 & 3199 & 3900 & 2401 \\
\hline 2011 & 2201 & 2600 & 3300 & 4099 & 2299 \\
\hline 2012 & 2199 & 2600 & 3600 & 4400 & 2500 \\
\hline 2013 & 2300 & 2700 & 3699 & 4516 & 2600 \\
\hline 2014 & 2600 & 2900 & 4200 & 4799 & 3000 \\
\hline 2015 & 2100 & 2400 & 4200 & 4900 & 2800 \\
\hline 2016 & 1700 & 2000 & 3999 & 4899 & 2499 \\
\hline 2017 & 1700 & 1900 & 4099 & 4799 & 2699 \\
\hline 2018 & 1300 & 1500 & 4100 & 4700 & 2300 \\
\hline 2019 & 1100 & 1399 & 3999 & 5099 & 2299 \\
\hline 2020 & 1201 & 1300 & 4199 & 5300 & 2700 \\
\hline
\end{tabular}


The EIA installed'nuclear capacity projections are shown in Figure 3.3 for the No New Orders Case, the Upper Reference Case and Lower Reference Case. The nuclear electric energy generation forecasts from EIA that correspond to the

TABLE 3.6. Cumulative Discharges from the Five Adjusted Data Bases (MTIHM)

\begin{tabular}{|c|c|c|c|c|c|}
\hline \multirow[b]{2}{*}{ Year } & \multicolumn{2}{|c|}{ No New Orders } & \multicolumn{2}{|c|}{ Upper Reference } & \multirow{2}{*}{$\begin{array}{c}\text { Lower } \\
\text { Reference }\end{array}$} \\
\hline & Ext. Burnup & Const. Burnup & Ext. Burnup & Const. Burnup & \\
\hline 1985 & 12651 & 12651 & 12651 & 12651 & 12651 \\
\hline 1986 & 14051 & 14051 & 14051 & 14051 & 14051 \\
\hline 1987 & 15551 & 15551 & 15600 & 15643 & 15551 \\
\hline 1988 & 17251 & 17251 & 17439 & 17443 & 17251 \\
\hline 1989 & 19151 & 19151 & 19539 & 19543 & 19151 \\
\hline 1990 & 21151 & 21151 & 21539 & 21743 & 21151 \\
\hline 1991 & 23251 & 23251 & 23538 & 23943 & 23251 \\
\hline 1992 & 25351 & 25451 & 25731 & 26242 & 25351 \\
\hline 1993 & 27451 & 27551 & 27731 & 28442 & 27451 \\
\hline 1994 & 29451 & 29651 & 29730 & 30649 & 29451 \\
\hline 1995 & 31450 & 31951 & 31830 & 33049 & 31450 \\
\hline 1996 & 33450 & 34150 & 33830 & 35549 & 33450 \\
\hline 1997 & 35250 & 36250 & 35830 & 37949 & 35250 \\
\hline 1998 & 37150 & 38550 & 37821 & 40448 & 37150 \\
\hline 1999 & 39150 & 40850 & 39821 & 42948 & 39150 \\
\hline 2000 & 41050 & 43050 & 41920 & 45448 & 41050 \\
\hline 2001 & 42950 & 45350 & 43920 & 47948 & 42950 \\
\hline 2002 & 44849 & 47650 & 45920 & 50457 & 44849 \\
\hline 2003 & 46850 & 49950 & 48120 & 53157 & 46850 \\
\hline 2004 & 48869 & 52273 & 50439 & 55981 & 48850 \\
\hline 2005 & 50749 & 54550 & 52720 & 58757 & 50749 \\
\hline 2006 & 52749 & 56849 & 55320 & 61857 & 52749 \\
\hline 2007 & 54849 & 59249 & 58020 & 65256 & 54849 \\
\hline 2008 & 56749 & 61649 & 60619 & 68656 & 56749 \\
\hline 2009 & 58849 & 64149 & 63719 & 72256 & 58948 \\
\hline 2010 & 61149 & 66749 & 66918 & 76156 & 61349 \\
\hline 2011 & 63350 & 69349 & 70218 & 80255 & 63648 \\
\hline 2012 & 65549 & 71949 & 73817 & 84655 & 66148 \\
\hline 2013 & 67849 & 74649 & 77517 & 89171 & 68748 \\
\hline 2014 & 70449 & 77549 & 81716 & 93970 & 71748 \\
\hline 2015 & 72549 & 79949 & 85916 & 98870 & 74548 \\
\hline 201 & 74249 & 81949 & 89915 & 103769 & 77047 \\
\hline 201 & 75949 & 83849 & 94014 & 108568 & 79746 \\
\hline 201 & 77249 & 85349 & 98114 & 113268 & 82046 \\
\hline 201 & 78349 & 86748 & 102113 & 118367 & 84345 \\
\hline 2020 & 79550 & 88048 & 106313 & 123667 & 87045 \\
\hline
\end{tabular}




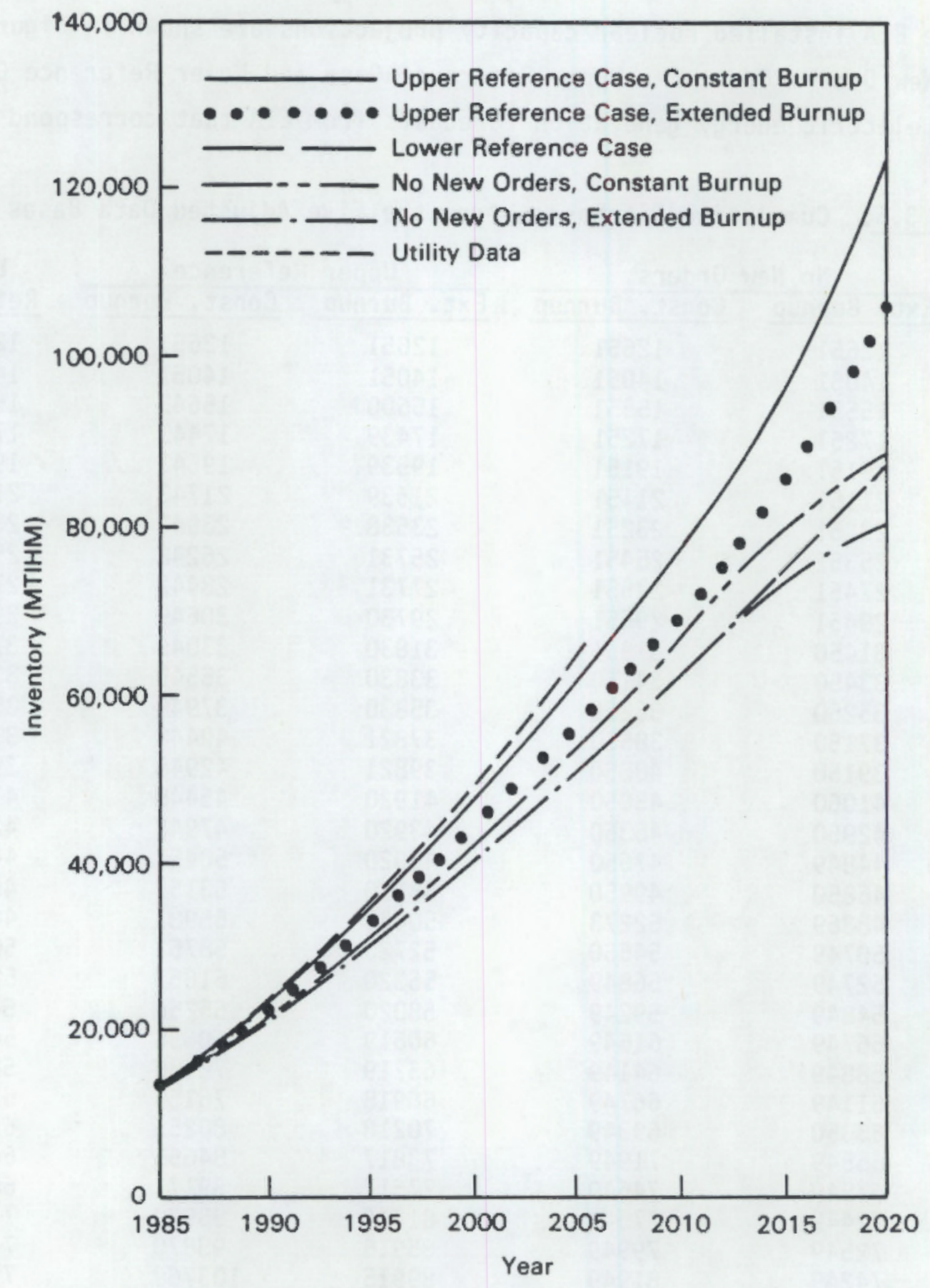

FIGURE 3.2. Projected Spent Fuel Inventory 


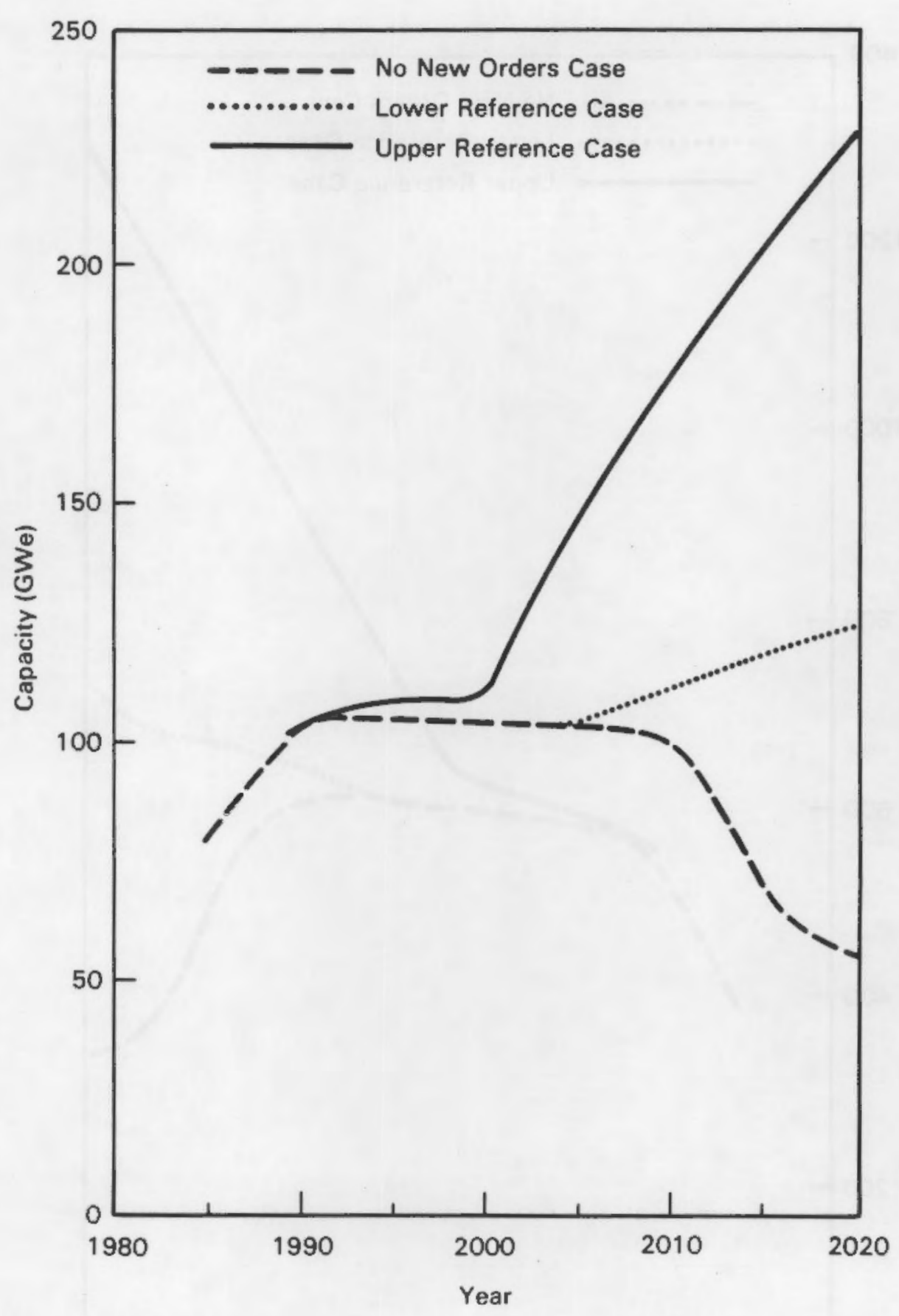

FIGURE 3.3. EIA Installed Nuclear Capacity Projections

capacity projections are shown in Figure 3.4. The forecasts of capacity and energy are given in five-year intervals from 1985 to 2020. The capacity and energy forecasts, unlike the spent-fuel discharge forecast, are independent of 


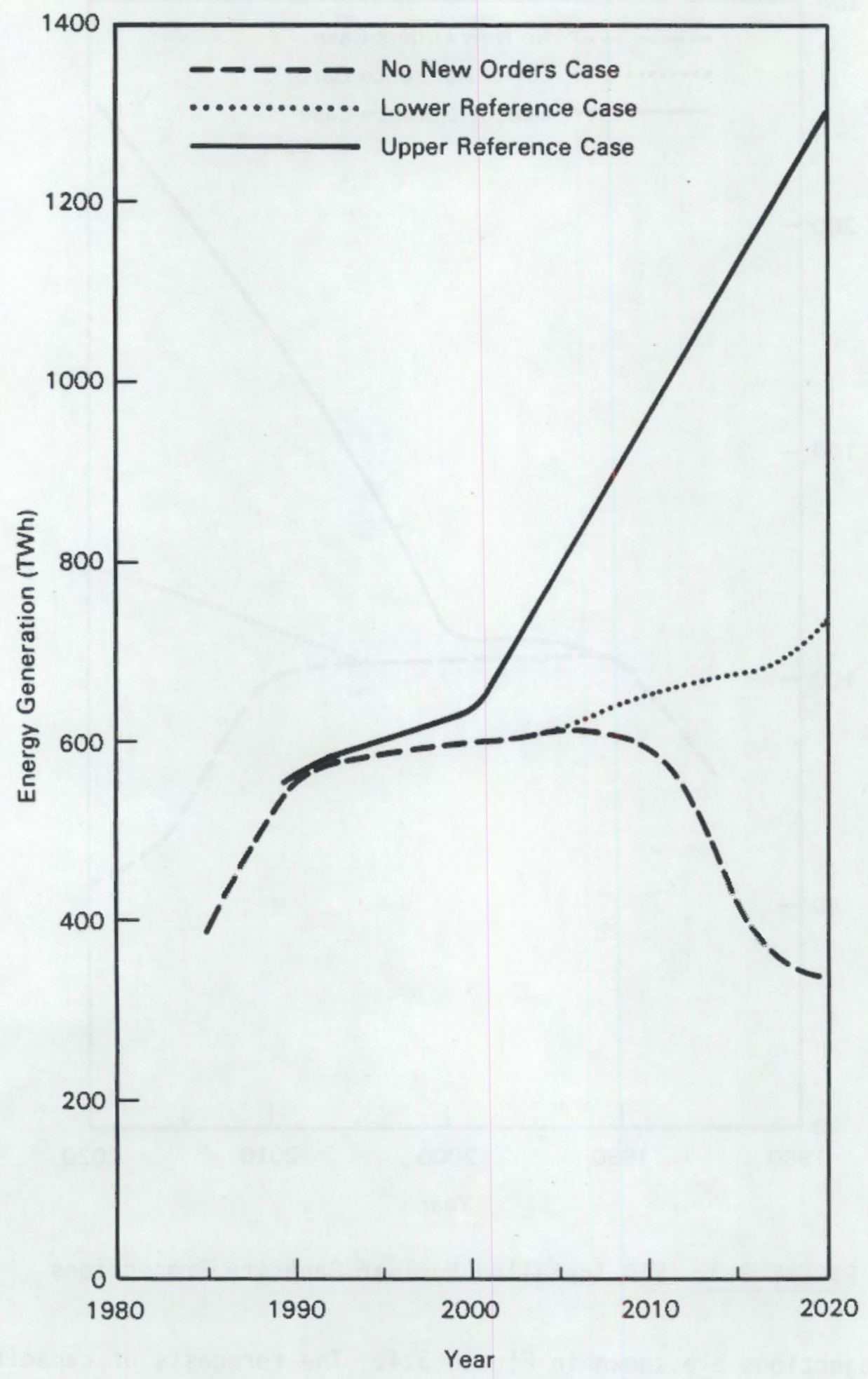

FIGURE 3.4. EIA Nuclear Energy Generation Projection 
the assumptions of extended or constant burnup. Therefore, only the three cases shown in Figures 3.3 and 3.4 are necessary.

Figure 3.5 shows the adjusted data base nuclear generation capacity compared to the EIA values shown in Figure 3.3. The EIA values are shown as points at the five year intervals which define the EIA projection. The EIA target capacities and data base capacities are nearly identical.

The energy generation from each ADB is compared to EIA five-year interval projections in Figure 3.6. The approximate nature of the energy calculation from data-hase discharges and hurnups shows up as a year-to-year variation in energy. Figure 3.6 shows that EIA projected values are well within variations in apparent annual energy generation and that the pattern corresponds well with EIA projections. Since the primary use of the adjusted data bases is to provide detailed projections of spent-fuel discharges, and not to forecast nuclear energy generation, the calculation shows that the amounts discharged and the burnup of the discharged fuel is sufficiently consistent with EIA energygeneration forecasts.

\subsection{SPENT FUEL STORAGE REQUIREMENTS}

The final result to be reported is the analysis of additional fuel storage requirements. The two storage-scenario cases covered in this analysis are based on the maximum at-reactor storage capacities of the individual reactor pools, as determined by the utilities. Both cases include allowances for maintaining full core discharge capability, also referred to as full core reserve (FCR), for each separate reactor. A single FCR is assumed to be maintained for all units at multiple reactor stations employing either a single common spent fuel storage pool, or separate pools with interconnections allowing spent fuel transfer between them. The only difference between the two cases is in the consideration of transshipment to other reactor sites. The first case assumes that transshipments occur only as currently planned by the utilities. The second case includes the additional assumption that there are no constraints on 


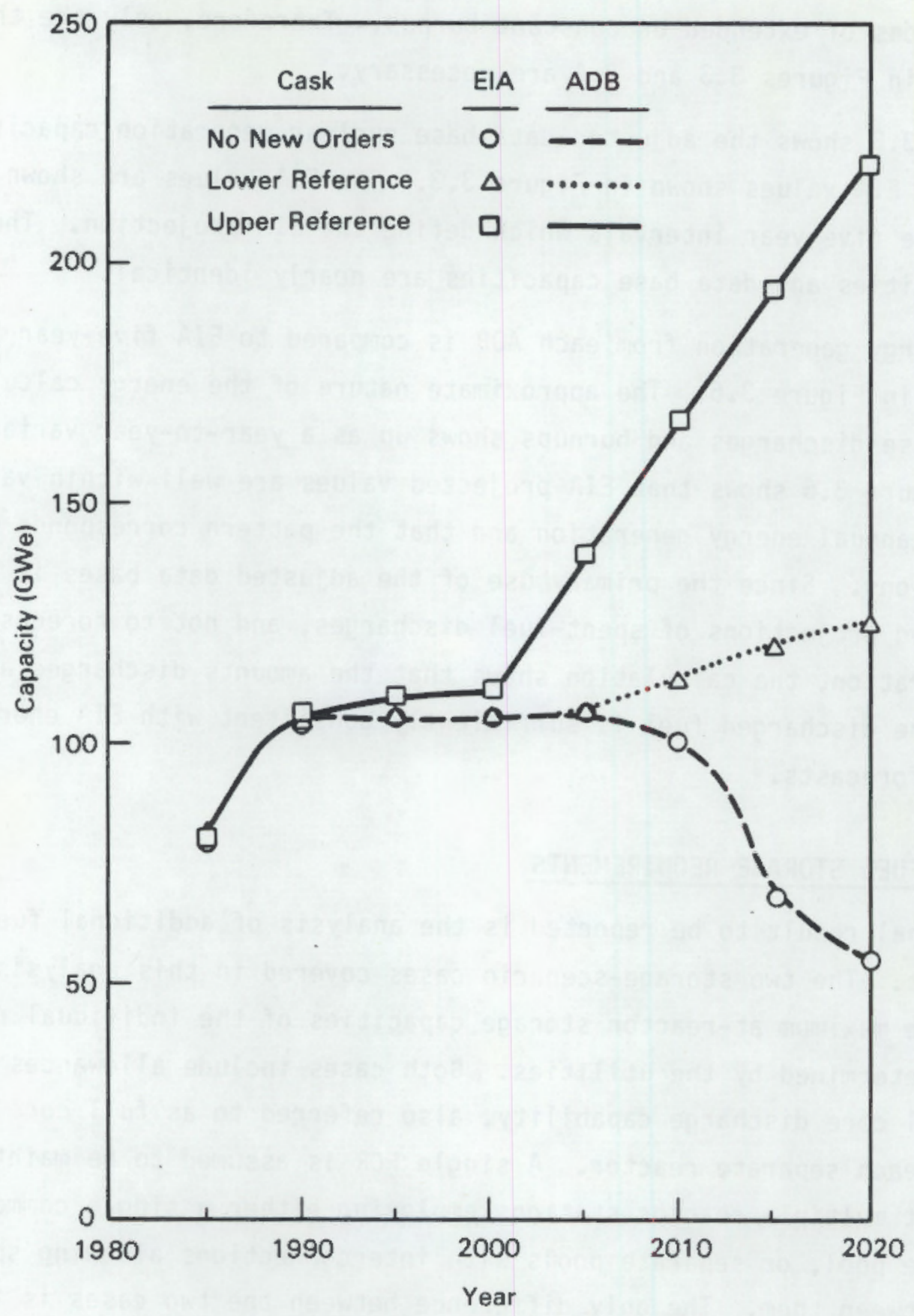

FIGURE 3.5. ADB Capacity and EIA Projections

transshipments of spent fuel among reactors of like type (i.e. among BWRs or among PWRs) within a given utility system. This allows unused spent fuel storage capacity at one reactor to offset needs for additional capacity at another 


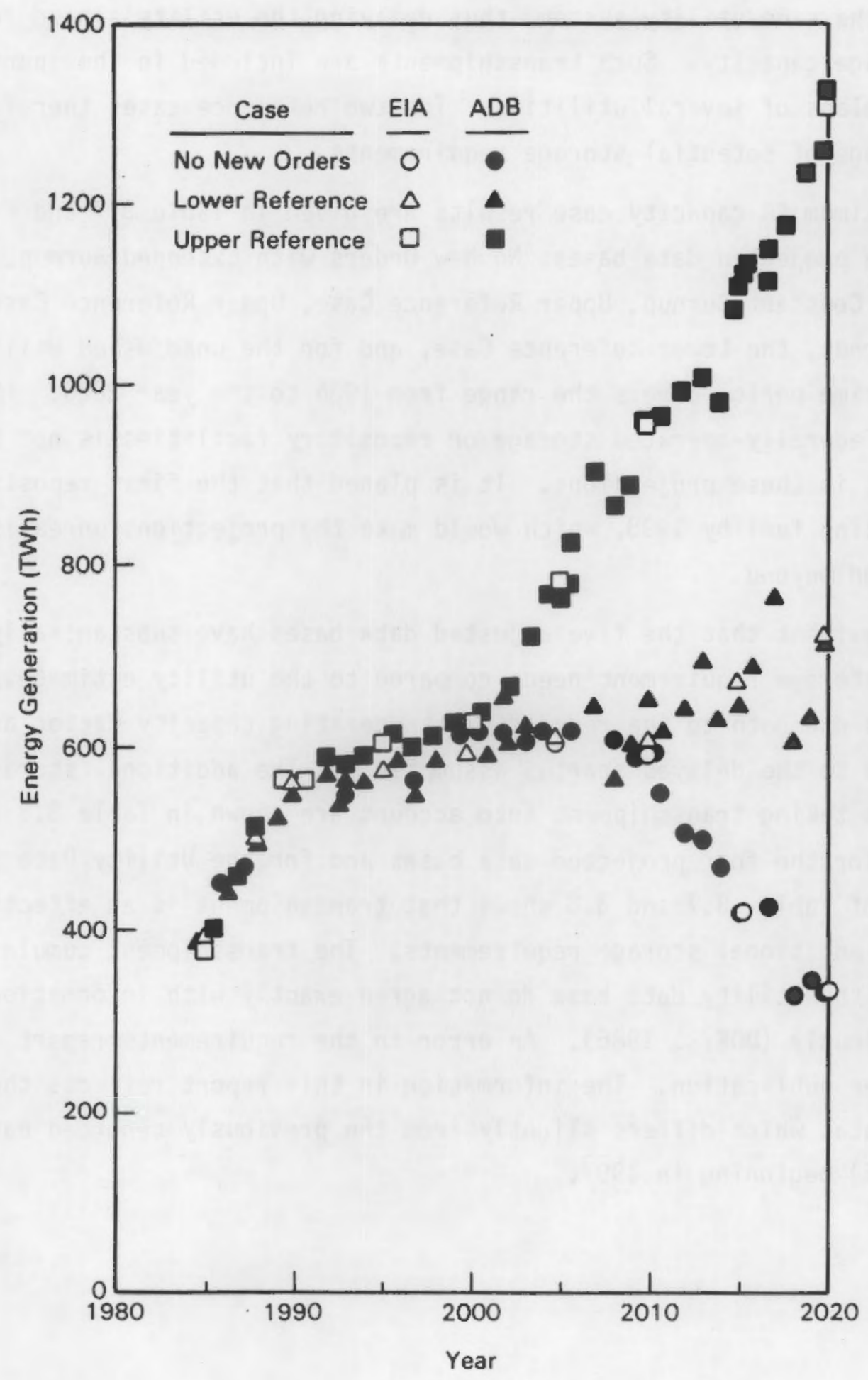

FIGURE 3.6. ADB Energy and EIA Energy Projections 
reactor in the same utility system, thus delaying the utility's need for additional storage capacity. Such transshipments are included in the spent-fuel management plans of several utilities. The two reference cases therefore define a range of potential storage requirements.

The maximum AR capacity case results are given in Table 3.7 and Figure 3.7 for the five projected data bases: No New Orders with Extended Burnup, No New Orders with Constant Burnup, Upper Reference Case, Upper Reference Case with Constant Burnup, the Lower Reference Case, and for the unadjusted Utility Data Base. The time period covers the range from 1986 to the year 2020. The availability of Federally-operated storage or repository facilities is not taken into account in these projections. It is planed that the first repository will begin accepting fuel by 1998 , which would make the projections unrealistic for that year and beyond.

It is evident that the five adjusted data bases have substantially reduced additional storage requirement needs compared to the utility estimate. This reduction is due both to the reduced plant operating capacity factor and (in later years) to the delayed startup assumptions. The additional storage requirements taking transshipment into account are shown in Table 3.8 and in Figure 3.8 for the four projected data bases and for the Utility Data Base. A comparison of Tables 3.7 and 3.8 shows that transshipment is an effective means of reducing additional storage requirements. The transshipment cumulative amounts for the utility data base do not agree exactly with information published previously (DOE/RL 1986). An error in the requirements report was discovered after publication. The information in this report reflects the corrected data, which differs slightly from the previously reported data (DOE/RL 1986) beginning in 1997. 
TABLE 3.7. Cumulative Storage Requirements--Maximum AR Storage (MTIHM)

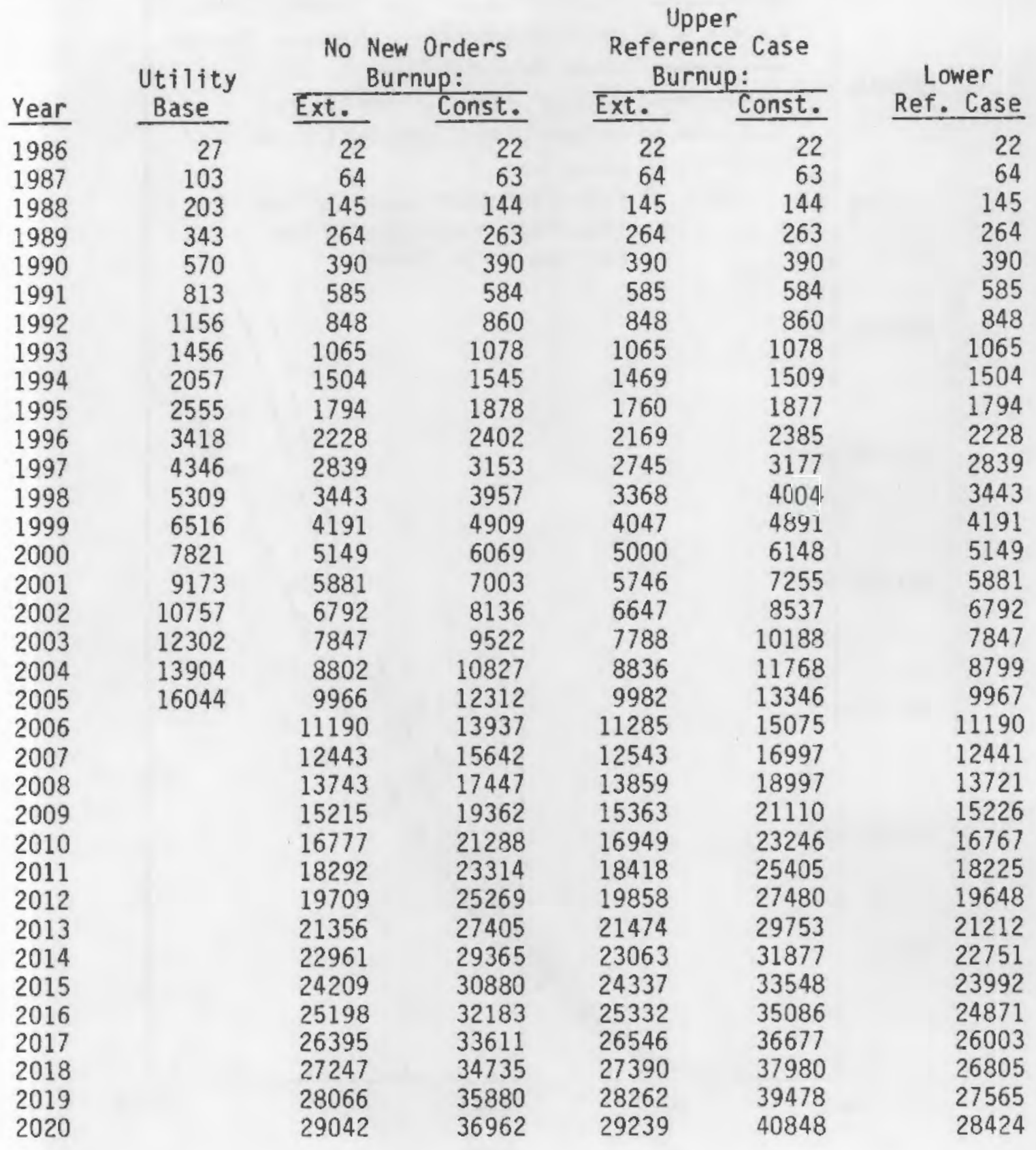




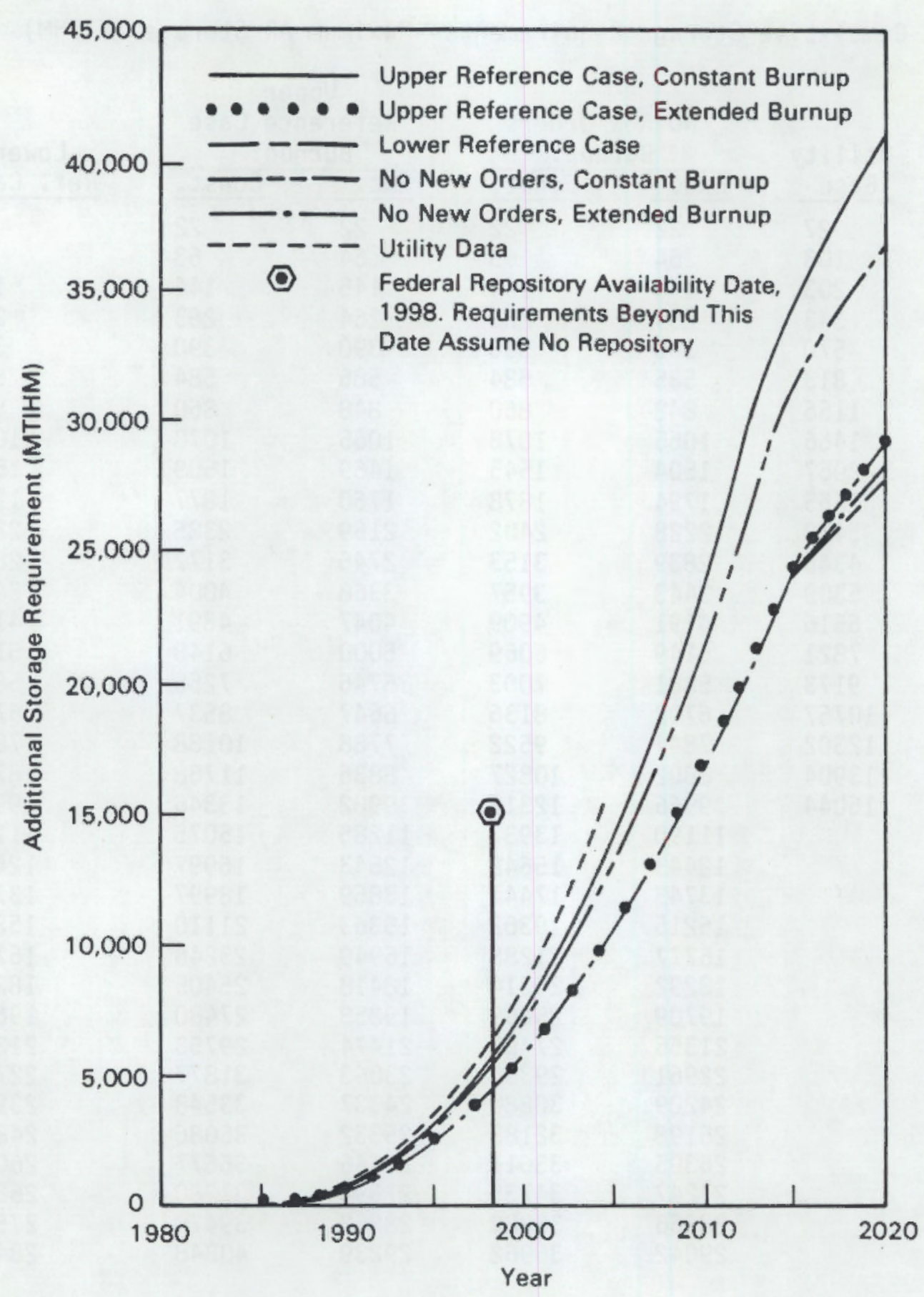

FIGURE 3.7. Cumulative Additional Storage Requirement--Maximum At-Reactor Storage 
TABLE 3.8. Cumulative Storage Requirements--Maximum AR Storage Plus Transshipment (MTIHM)

\begin{tabular}{|c|c|c|c|c|c|c|}
\hline \multirow[b]{2}{*}{ Year } & \multirow{2}{*}{$\begin{array}{l}\text { Utility } \\
\text { Base } \\
\end{array}$} & \multicolumn{2}{|c|}{$\begin{array}{c}\text { No New Orders } \\
\text { Burnup: }\end{array}$} & \multicolumn{2}{|c|}{$\begin{array}{c}\text { Upper } \\
\text { Reference Case } \\
\text { Burnup: }\end{array}$} & \multirow{2}{*}{$\begin{array}{l}\text { Lower } \\
\text { Ref. Case }\end{array}$} \\
\hline & & Ext. & Const. & Ext. & Const. & \\
\hline 1986 & 0 & 0 & 0 & 0 & 0 & 0 \\
\hline 1987 & 31 & 17 & 16 & 17 & 16 & 17 \\
\hline 1988 & 31 & 17 & 16 & 17 & 16 & 17 \\
\hline 1989 & 93 & 70 & 69 & 70 & 69 & 70 \\
\hline 1990 & 121 & 92 & 92 & 92 & 92 & 92 \\
\hline 1991 & 191 & 126 & 126 & 126 & 126 & 126 \\
\hline 1992 & 292 & 162 & 163 & 162 & 163 & 162 \\
\hline 1993 & 515 & 253 & 258 & 199 & 202 & 253 \\
\hline 1994 & 926 & 495 & 520 & 342 & 359 & 495 \\
\hline 1995 & 1333 & 696 & 748 & 523 & 588 & 696 \\
\hline 1996 & 2086 & 987 & 1131 & 760 & 986 & 987 \\
\hline 1997 & 2765 (a) & 1434 & 1670 & 1252 & 1609 & 1434 \\
\hline 1998 & 3746 (a) & 1919 & 2328 & 1716 & 2265 & 1919 \\
\hline 1999 & 4871 (a) & 2551 & 3224 & 2289 & 3114 & 2551 \\
\hline 2000 & $6029^{(a)}$ & 3371 & 4206 & 3145 & 4212 & 3371 \\
\hline 2001 & 7310 & 4112 & 5188 & 3880 & 5280 & 4112 \\
\hline 2002 & 8777 & 4951 & 6225 & 4725 & 6436 & 4951 \\
\hline 2003 & 10219 & 5945 & 7479 & 5782 & 7964 & 5945 \\
\hline 2004 & 11847 & 6824 & 8646 & 6733 & 9465 & 6821 \\
\hline 2005 & 13997 & 7887 & 10027 & 7752 & 11052 & 7888 \\
\hline 2006 & & 9001 & 11514 & 8920 & 12717 & 9004 \\
\hline 2007 & & 10094 & 13244 & 10060 & 14769 & 10097 \\
\hline 2008 & & 11393 & 15101 & 11335 & 16833 & 11363 \\
\hline 2009 & & 12859 & 17070 & 12766 & 18837 & 12870 \\
\hline 2010 & & 14482 & 19168 & 14358 & 21155 & 14450 \\
\hline 2011 & & 16036 & 21349 & 15854 & 23401 & 15926 \\
\hline 2012 & & 17518 & 23459 & 17296 & 25591 & 17415 \\
\hline 2013 & & 19248 & 25730 & 18949 & 27897 & 19035 \\
\hline 2014 & & 21026 & 27864 & 20651 & 30025 & 20737 \\
\hline 2015 & & 22376 & 29502 & 22014 & 31873 & 22078 \\
\hline 2016 & & 23516 & 30946 & 23175 & 33539 & 23079 \\
\hline 2017 & & 24801 & 32422 & 24414 & 35166 & 24285 \\
\hline 2018 & & 25081 & 33612 & 25411 & 36519 & 25221 \\
\hline 2019 & & 26724 & 34823 & 26381 & 38056 & 26069 \\
\hline 2020 & & 27775 & 35969 & 27425 & 39512 & 27074 \\
\hline
\end{tabular}

(a) Requirements differ from those reported by DOE/RL (1986) for the four year period 1997-2000 due to a processor error which was discovered and corrected after that report was issued. By 2000 the difference is 80 MTIHM out of 6029 , or $1.3 \%$. 


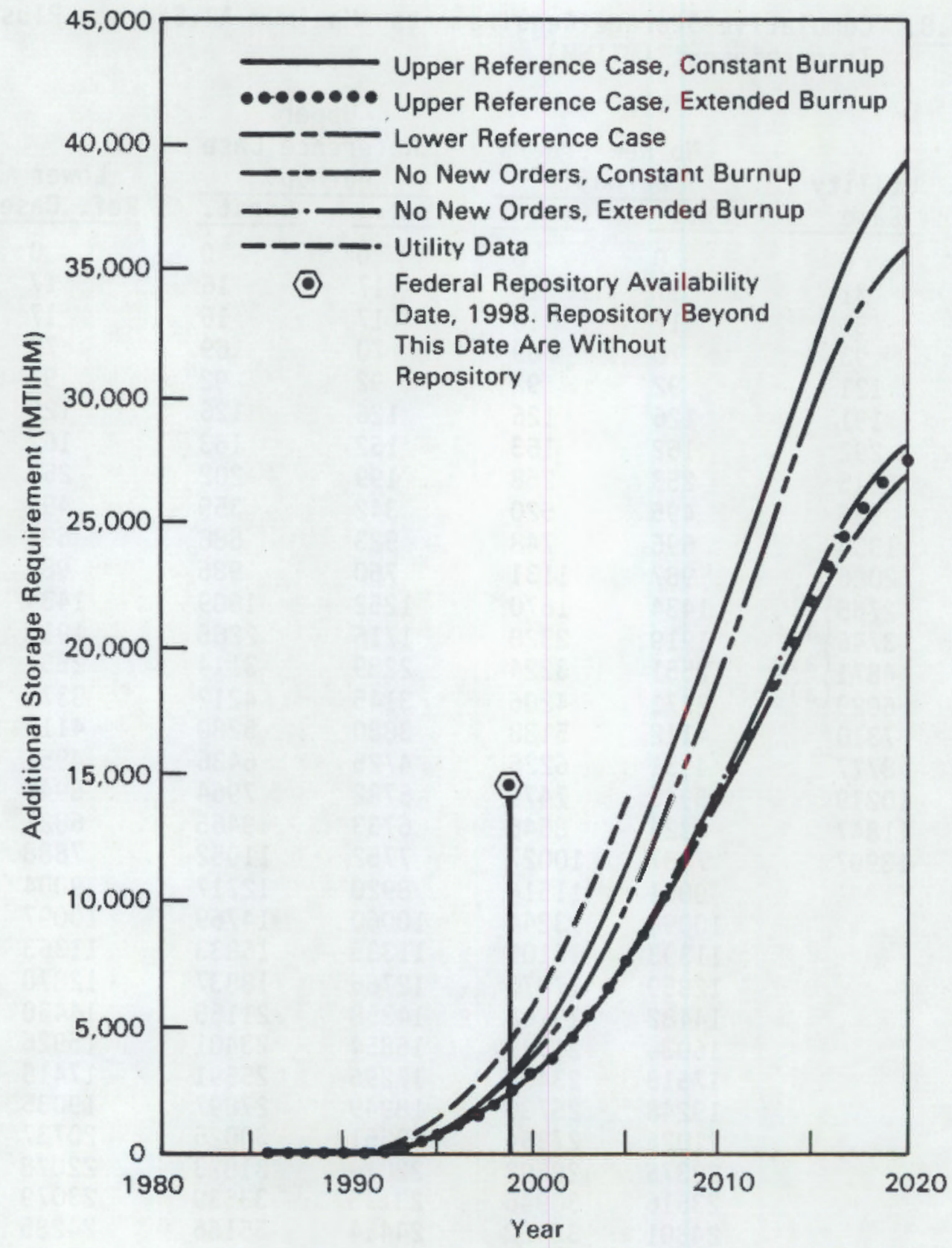

FIGURE 3.8. Cumulative Additional Storage Requirement--Maximum At-Reactor Storage Plus Transshipment 


\subsection{REFERENCES}

DOE/EIA. 1986a. Commercial Nuclear Power: Prospects for the United States and the World. DOE/EIA-0438(86), U.S. Department of Energy, Washington, D.C.

DOE/EIA. 1986b. World Nuclear Fuel Cycle Requirements 1986. DOE/EIA0436(86), U.S. Department of Energy, Washington, DC.

DOE/RL. 1986. Spent Fuel Storage Requirements: An Update of DOE/RL-85-2. DOE/RL-86-5, U.S. Department of Energy, Richland Operations office, Rich? and, Washington.

Heeb, C. M., R. A. Libby and G. M. Holter. 1985. Reactor-Specific Spent Fue 1 Discharge Projections: 1984 to 2020. PNL-5396, Pacific Northwest Laboratory, Richland, Washington.

Heeb, C. M., R. A. Libby, R. C. Walling, W. L. Purcell. 1986. ReactorSpecific Spent Fuel Discharge Projections, 1985 to 2020 . PNL-5833, Pacific Northwest Laboratory, Richland, Washington.

Holter, G. M., W. L. Purce11, M. E. Schutz, J. R. Young. 1986. A Method for Assigning Sites to Projected Generic Nuclear Power Plants. PNL-5870, Pacific Northwest Laboratory, Richland, Washington. 


\section{-}


APPENDIX A

DETAILED DATA BASE RESULTS 
APPENDIX A

DETAILED DATA BASE RESULTS

The following tables provide detailed results for four scenarios: Utility, No New Orders, Upper Reference Case, and Lower Reference Case. Table A.1 gives startup and shutdown dates for Utility, No New Orders, Upper Reference, and Lower Reference Cases. Table A.2 gives names and startup dates for the Upper and Lower Reference Case generic reactors. Tables A.3 to A.8 give Upper Reference Case by-reactor discharge projections similar to information supplied by the Spent Fuel Storage Requirements Report for the Utility Data Base (DOE 1986c). The Upper Reference Case has been selected by OCRWM as the planing base case. Therefore, Tables A.3 through A.8 are provided for this case.

Tahle

Number

A.1

A.2 Startup and Shutdown Dates with Federal Energy Region shown for Upper and Lower Reference Case Generic Reactors

A.3 Upper Reference Case, 1985 Inventory and Projected Annual Reactor Discharges

A.4 Upper Reference Case, 1985 and Projected Inventories

A.5 Upper Reference Case, Maximum At-Reactor Capacity - Projected Annual Storage Requirements

A.6 Upper Reference Case, Maximum At-Reactor Capacity - Projected Cumulative Storage Requirements

A.7 Upper Reference Case, Maximum At-Reactor Capacity - Plus Transshipment - Projected Annual Storage Requirements

A.8 Upper Reference Case, Maximum At-Reactor Capacity - Plus Transshipment - Projected Cumulative Storage Requi rements 
TABLE A.1. Startup and Shutdown Dates with Federa1 Energy Region Shown for Utility, No New Orders, Upper Reference and Lower Reference Cases

\begin{tabular}{|c|c|c|c|c|c|c|c|}
\hline \multirow[b]{2}{*}{ Reactor } & \multirow{2}{*}{$\begin{array}{l}\text { Fed. En. } \\
\text { Region }\end{array}$} & \multirow{2}{*}{$\frac{\text { Utility }}{\text { SU }}$} & \multirow{2}{*}{$\frac{\text { Case }}{\text { SD }}$} & \multicolumn{2}{|c|}{$\begin{array}{c}\text { No New } \\
\text { Orders and } \\
\text { Lower } \\
\text { Ref. Cases }\end{array}$} & \multicolumn{2}{|c|}{$\begin{array}{c}\text { Upper } \\
\text { Ref. Case }\end{array}$} \\
\hline & & & & SU & SD & & $\mathrm{SD}$ \\
\hline DRESDEN 1 & 5 & $1960 / 07$ & 1984 & $1960 / 07$ & 1978 & $1960 / 07$ & 1978 \\
\hline YANKEE-RDWE & 1 & $1961 / 07$ & 2001 & $1961 / 07$ & 1998 & $1961 / 07$ & 1998 \\
\hline INDIAN PT 1 & 2 & $1962 / 10$ & 1980 & $1962 / 10$ & 1974 & $1962 / 10$ & 1974 \\
\hline HUMBOLDT BAY & 9 & $1963 / 09$ & 1976 & $1963 / 09$ & 1976 & $1963 / 09$ & 1976 \\
\hline BIG ROCK POINT & 5 & $1965 / 11$ & 2001 & $1965 / 11$ & 2005 & $1965 / 11$ & 2005 \\
\hline HADDAM NECK & 1 & $1968 / 01$ & 2004 & $1968 / 01$ & 2008 & $1968 / 01$ & 2008 \\
\hline SAN ONOFRE 1 & 9 & $1968 / 01$ & 1999 & $1968 / 01$ & 2008 & $1968 / 01$ & 2008 \\
\hline LACROSSE & 5 & $1969 / 11$ & 2002 & $1969 / 11$ & 2009 & $1969 / 11$ & 2009 \\
\hline OYSTER CREEK & 2 & $1969 / 12$ & 2004 & $1969 / 12$ & 2009 & $1969 / 12$ & 2009 \\
\hline NINE MILE PT 1 & 2 & $1969 / 12$ & 2005 & $1970 / 04$ & 2010 & $1970 / 04$ & 2010 \\
\hline DRESDEN 2 & 5 & $1970 / 06$ & 2008 & $1970 / 06$ & 2010 & $1970 / 06$ & 2010 \\
\hline GINNA & 2 & $1970 / 07$ & 2006 & $1970 / 07$ & 2010 & $1970 /$ & 2010 \\
\hline MILLSTONE 1 & 1 & $1970 / 12$ & 2010 & $1970 / 12$ & 2010 & $1970 / 12$ & 2010 \\
\hline POINT BEACH 1 & 5 & $1970 / 12$ & 2007 & $1970 / 12$ & 2010 & $1970 / 12$ & 2010 \\
\hline ROB INSON & 4 & $1971 / 03$ & 2007 & $1971 / 03$ & 2011 & $1971 /$ & 2011 \\
\hline MONT ICELLO & 5 & 197 & 2007 & $1971 / 06$ & 2011 & 1971 & 2011 \\
\hline DRE SDEN 3 & 5 & 197 & 2006 & $1971 / 11$ & 2011 & 19 & 2011 \\
\hline PAL I SADES & 5 & $1971 / 12$ & 2011 & $1971 / 12$ & 2011 & $1971 /$ & 2011 \\
\hline POINT BEACH 2 & 5 & $1972 / 10$ & 2008 & $1972 / 10$ & 2012 & $1972 / 10$ & 2012 \\
\hline VT YANKEE & 1 & $1972 / 11$ & 2012 & $1972 / 11$ & 2012 & $1972 / 11$ & 2012 \\
\hline PILGRIM 1 & 1 & $1972 / 12$ & 2008 & $1972 / 12$ & 2012 & $1972 / 12$ & 2012 \\
\hline TURKEY PT 3 & 4 & $1972 / 12$ & 2007 & $1972 / 12$ & 2012 & $1972 / 12$ & 2012 \\
\hline MAINE YANKEE & 1 & $1972 / 12$ & 2008 & $1972 / 12$ & 2012 & $1972 / 12$ & 2012 \\
\hline SURRY 1 & 3 & $1972 / 12$ & 2008 & $1972 / 12$ & 2012 & $1972 / 12$ & 2012 \\
\hline QUAD CITIES I & 5 & $1973 / 02$ & 2007 & $1973 / 02$ & 2013 & $1973 / 02$ & 2013 \\
\hline QUAD CITIES 2 & 5 & $1973 / 03$ & 2007 & $1973 / 03$ & 2013 & $1973 / 03$ & 2013 \\
\hline SURRY 2 & 3 & $1973 / 05$ & 2008 & $1973 / 05$ & 2013 & $1973 / 05$ & 2013 \\
\hline OCONEE 1 & 4 & $1973 / 07$ & 2013 & $1973 / 07$ & 2013 & $1973 / 07$ & 2013 \\
\hline TURKEY PT 4 & 4 & $1973 / 09$ & 2007 & $1973 / 09$ & 2013 & $1973 / 09$ & 2013 \\
\hline FORT CALHOON & 7 & $1973 / 09$ & 2008 & $1973 / 09$ & 2013 & $1973 / 09$ & 2013 \\
\hline ZION 1 & 5 & $1973 / 12$ & 2007 & $1973 / 12$ & 2013 & $1973 / 12$ & 2013 \\
\hline PRAIRIE IS 1 & 5 & $1973 / 12$ & 2008 & $1973 / 12$ & 2013 & $1973 / 12$ & 2013 \\
\hline 3 MILE IS 1 & 3 & $1974 / 06$ & 2008 & $1974 / 06$ & 2014 & $1974 / 06$ & 2014 \\
\hline COOPER ST & 9 & $1974 / 06$ & 2004 & $1974 / 06$ & 2014 & $1974 / 06$ & 2014 \\
\hline KEWAUNEE & 5 & $1974 / 06$ & 2014 & $1974 / 06$ & 2014 & $1974 / 06$ & 2014 \\
\hline PEACHBOTTOM 2 & 3 & $1974 / 07$ & 2008 & $1974 / 07$ & 2014 & $1974 / 07$ & 2014 \\
\hline INDIAN PT 2 & 2 & $1974 / 08$ & 2006 & $1974 / 08$ & 2014 & $1974 / 08$ & 2014 \\
\hline BROWNS FERRY 1 & 4 & $1974 / 08$ & 2014 & $1974 / 08$ & 2014 & $1974 / 08$ & 2014 \\
\hline ZION 2 & 5 & $1974 / 09$ & 2008 & $1974 / 09$ & 2014 & $1974 / 09$ & 2014 \\
\hline OCONEE 2 & 4 & $1974 / 09$ & 2013 & $1974 / 09$ & 2014 & $1974 / 09$ & 2014 \\
\hline ARK NUCLEAR 1 & 6 & $1974 / 12$ & 2008 & $1974 / 12$ & 2014 & $1974 / 12$ & 2014 \\
\hline OCONEE 3 & 4 & $1974 / 12$ & 2014 & $1974 / 12$ & 2014 & $1974 / 12$ & 2014 \\
\hline HATCH 1 & 4 & $1974 / 12$ & 2009 & $1974 / 12$ & 2014 & $1974 / 12$ & 2014 \\
\hline
\end{tabular}


TABLE A.1. Startup and Shutdown Oates with Federal Energy Region Shown for Utility, No New Brders, Upper Reference and Lower Reference Cases (cont'd)

\begin{tabular}{|c|c|c|c|c|c|c|c|}
\hline Reactor & $\begin{array}{l}\text { Fed. En. } \\
\text { Region } \\
\end{array}$ & $\frac{\text { Utility }}{\text { SU }}$ & $\frac{\text { Case }}{\text { SO }}$ & $\begin{array}{r}\text { No N } \\
\text { Orders } \\
\text { Low } \\
\text { Ref. C } \\
\text { SU }\end{array}$ & $\begin{array}{l}\text { ind } \\
\text { SD } \\
\end{array}$ & $\begin{array}{l}\text { Ref } \\
\overline{S U} \\
\end{array}$ & $\begin{array}{l}\text { se } \\
50\end{array}$ \\
\hline PRAIRIE IS 2 & 5 & $1974 / 12$ & 2008 & $1974 / 12$ & 2014 & $1974 / 12$ & 2014 \\
\hline HBOTTOM 3 & 3 & $1974 / 12$ & 2008 & $1974 / 12$ & 2014 & $1974 / 12$ & 2014 \\
\hline DUANE ARNOLD & 7 & $1975 / 02$ & 2010 & $1975 / 02$ & 2015 & $1975 / 02$ & 2015 \\
\hline BROWNS FERRY 2 & 4 & 197 & 2014 & $1975 / 03$ & 2015 & $1975 / 03$ & 2015 \\
\hline RANCHO SECO 1 & 9 & 197 & 2008 & $1975 / 04$ & 2015 & $1975 / 04$ & 2015 \\
\hline CALVERT CLIFFS 1 & 3 & $1975 / 05$ & 2014 & $1975 / 05$ & 2015 & $1975 / 05$ & 2015 \\
\hline FIPZPATRICK & 2 & $1975 / 07$ & 2015 & $1975 / 07$ & 2015 & $1975 / 07$ & 2015 \\
\hline COOK 1 & 5 & 108 & 2009 & j/08 & 2015 & $1975 / 08$ & 2015 \\
\hline BRUNSWICX 1 & 4 & $1975 / 11$ & 2010 & $1975 / 11$ & 2015 & $1975 / 11$ & 2015 \\
\hline MILLSTONE 2 & 1 & $1975 / 12$ & 2015 & $1975 / 12$ & 2015 & $1975 / 12$ & 2015 \\
\hline TROJAN & 10 & $1976 / 05$ & 2011 & $1976 / 05$ & 2016 & $1976 / 05$ & 2016 \\
\hline INDIAN PT 3 & 2 & $1976 / 08$ & 2015 & $1976 / 08$ & 2016 & $1976 / 08$ & 2016 \\
\hline B VALLEY 1 & 3 & $1976 / 10$ & 2010 & $1976 / 10$ & 2016 & $1976 / 10$ & 2016 \\
\hline ST LUCIE 1 & 4 & $1976 / 12$ & 2010 & $1976 / 12$ & 2016 & $1976 / 12$ & 2016 \\
\hline BRUNSWICK 2 & 4 & $1977 / 03$ & 2010 & $1977 / 03$ & 2009 & $1977 / 03$ & 2009 \\
\hline CRYSTAL R 3 & 4 & $1977 / 03$ & 2016 & $1977 / 03$ & 2017 & $1977 / 03$ & 2017 \\
\hline BROWNS FERRY 3 & 4 & $1977 / 03$ & 2017 & $1977 / 03$ & 2017 & $1977 / 03$ & 2017 \\
\hline CALVERT CLIFFS 2 & 3 & $1977 / 04$ & 2016 & $1977 / 04$ & 2017 & $1977 / 04$ & 2017 \\
\hline SALEM 1 & 2 & $1977 / 06$ & 2016 & $1977 / 06$ & 2017 & $1977 / 06$ & 2017 \\
\hline FARLEY 1 & 4 & $1977 / 12$ & 2012 & $1977 / 12$ & 2017 & $1977 / 12$ & 2017 \\
\hline NORTH ANNA 1 & 3 & $1978 / 06$ & 2011 & $1978 / 06$ & 2018 & $1978 / 06$ & 2018 \\
\hline $\operatorname{cook} 2$ & 5 & 197 & 2009 & $1978 / 07$ & 2018 & $1978 / 07$ & 2018 \\
\hline DAVIS-BESSE 1 & 5 & & 2017 & $1978 / 07$ & 2018 & $1978 / 07$ & 2018 \\
\hline FT ST VRAIN & 8 & 197 & 2007 & $1979 / 07$ & 2007 & $1979 / 07$ & 2007 \\
\hline HATCH 2 & 4 & & 2012 & $1979 / 09$ & 2019 & $1979 / 09$ & 2019 \\
\hline ARX NUCLEAR 2 & 6 & & 20 & $/ 03$ & 2020 & $0 / 03$ & 2020 \\
\hline ANNA 2 & 3 & & & $/ 12$ & 2020 & $0 / 12$ & 2020 \\
\hline FAR & 4 & & & & 2021 & $1 / 07$ & 2021 \\
\hline $\mathrm{AH} 1$ & 4 & & & & 2021 & & 2021 \\
\hline RE 1 & 4 & $1981 / 09$ & 2021 & & 2021 & & 021 \\
\hline SAl. & 2 & & & $1 / 10$ & 2021 & $/ 10$ & 2021 \\
\hline SEQUOYAH 2 & 4 & 106 & 2022 & & 2022 & 106 & 22 \\
\hline LE 1 & 5 & & 20 & 110 & 2022 & $/ 10$ & 2022 \\
\hline SUSQUEHANNA 1 & 3 & & 20 & $/ 06$ & 2023 & $3 / 06$ & 2023 \\
\hline ST LUCIE 2 & 4 & & 20 & $/ 08$ & 2027 & $3 / 08$ & 2023 \\
\hline SAN ONOFRE 2 & 9 & & & 108 & 2023 & $83 / 08$ & 2023 \\
\hline SUMMER 1 & 4 & $/ 01$ & 2024 & $/ 01$ & 2024 & $84 / 01$ & 2024 \\
\hline MCGUIRE 2 & 4 & & 2023 & $1984 / 02$ & 2024 & $1984 / 02$ & 2024 \\
\hline SAN ONOFR 3 & 9 & $1984 / 04$ & 2013 & $1984 / 04$ & 2024 & $1984 / 04$ & 2024 \\
\hline LASALLE 2 & 5 & $1984 / 09$ & 2023 & $1984 / 09$ & 2024 & $1984 / 09$ & 2024 \\
\hline & 7 & & 2024 & $1984 / 12$ & 2024 & $1984 / 12$ & 2024 \\
\hline WASH NUCLEAR 2 & 10 & $1984 / 12$ & 2023 & $1984 / 12$ & 2024 & $1984 / 12$ & 2024 \\
\hline
\end{tabular}


JABLE A.1. Startup and Shutdown Dates with Federal Energy Region Shown for Utility, No New Orders, Upper Reference and Lower Reference Cases (cont 'd)

\begin{tabular}{|c|c|c|c|c|c|c|c|}
\hline Reactor & $\begin{array}{l}\text { Fed. En. } \\
\text { Region }\end{array}$ & $\frac{\text { Utility }}{\text { SU }}$ & $\frac{\text { Case }}{\mathrm{SO}}$ & \multicolumn{2}{|c|}{$\begin{array}{c}\text { No New } \\
\text { Orders and } \\
\text { Lower } \\
\text { Ref. Cases }\end{array}$} & \multicolumn{2}{|c|}{$\begin{array}{c}\text { Upper } \\
\text { Ref. Case }\end{array}$} \\
\hline SUSQUEHANNA 2 & 3 & $1985 / 02$ & 2024 & $1985 / 02$ & 2025 & $1985 / 02$ & 2025 \\
\hline DIABLO CANYON 1 & 9 & $1985 / 05$ & 2025 & $1985 / 05$ & 2025 & $1985 / 05$ & 2025 \\
\hline ENRICO FERMI 2 & 5 & $1985 / 06$ & 2025 & $1985 / 06$ & 2025 & $1985 / 06$ & 2025 \\
\hline CATAWBA 1 & 4 & $1985 / 06$ & 2025 & $1985 / 06$ & 2025 & $1985 / 06$ & 2025 \\
\hline GRAND GULF 1 & 4 & $1985 / 07$ & 2022 & $1985 / 07$ & 2025 & $1985 / 07$ & 2025 \\
\hline BYRON 1 & 5 & $1985 / 09$ & 2024 & $1985 / 09$ & 2025 & $1985 / 09$ & 2025 \\
\hline WOLF CREEK & 7 & $1985 / 09$ & 2025 & $1985 / 09$ & 2025 & $1985 / 09$ & 2025 \\
\hline WATERFORD & 6 & $1985 / 09$ & 2024 & $1985 / 09$ & 2025 & $1985 / 09$ & 2025 \\
\hline PALO VERDE I & 9 & $1986 / 01$ & 2024 & $1986 / 01$ & 2026 & $1986 / 01$ & 2026 \\
\hline LIMER ICK 1 & 3 & $1986 / 02$ & 2024 & $1986 / 02$ & 2026 & $1986 / 02$ & 2026 \\
\hline RIVER BEND & 6 & $1986 / 03$ & 2025 & $1986 / 03$ & 2026 & $1986 / 03$ & 2026 \\
\hline DIABLO CANYON-2 & 9 & $1985 / 06$ & 2025 & $1986 / 04$ & 2026 & $1986 / 04$ & 2026 \\
\hline SHOREHAM & 2 & $1983 / 06$ & 2025 & $1988 / 08$ & 2028 & 1986 & 2026 \\
\hline PERRY 1 & 5 & $1986 / 04$ & 2026 & $1987 / 03$ & 2027 & 1986 & 2026 \\
\hline MILLSTONE 3 & 1 & $1986 / 05$ & 2025 & $1987 / 03$ & 2027 & 1986 & 2026 \\
\hline PALO VERDE 2 & 9 & $1986 / 08$ & 2025 & $1987 / 06$ & 2027 & 1987 & 2027 \\
\hline HOPE CREEK & 2 & $1986 / 04$ & 2026 & $1987 / 05$ & 2027 & 198 & 2027 \\
\hline CATAWBA 2 & 4 & 104 & 2026 & $37 / 06$ & 2027 & & 2027 \\
\hline NCHE 1 & 6 & 109 & 2028 & $3 / 03$ & 2028 & & 2027 \\
\hline DWOOD 1 & 5 & 102 & 2026 & $7 / 08$ & 2027 & & 2027 \\
\hline HARR IS 1 & 4 & 112 & 2017 & $7 / 09$ & 2027 & & 2027 \\
\hline 2 & 5 & 102 & 2026 & $7 / 10$ & 2027 & 19 & 2027 \\
\hline CLINTON 1 & 5 & $5 / 07$ & 2026 & $1987 / 11$ & 2027 & $1987 / 07$ & 2027 \\
\hline SEABROOK & 1 & $6 / 06$ & 2026 & $1988 / 02$ & 2028 & $1987 / 10$ & 2028 \\
\hline NINE MILE 2 & 2 & $6 / 08$ & 2026 & $1988 / 06$ & 2028 & $1987 / 12$ & 2027 \\
\hline VOGTLE 1 & 4 & $1987 / 02$ & 2027 & $8 / 06$ & 2028 & $1988 / 01$ & 2028 \\
\hline WATTS BAR 1 & 4 & $5 / 06$ & 2025 & $6 / 07$ & 2026 & $8 / 01$ & 2028 \\
\hline PALO VERDE 3 & 9 & $7 / 05$ & 2026 & $8 / 07$ & 2028 & $8 / 03$ & 2028 \\
\hline B VALLEY 2 & 3 & $7 / 06$ & 2026 & $8 / 06$ & 2028 & $38 / 04$ & 2028 \\
\hline SOUTH TEX UNIT 1 & 6 & $7 / 10$ & 2027 & $8 / 10$ & 2028 & $1988 / 04$ & 2028 \\
\hline BRAIDWO00 2 & 5 & $7 / 06$ & 2027 & $38 / 10$ & 2028 & $1988 / 06$ & 2028 \\
\hline COMANCHE 2 & 6 & 709 & 2028 & $89 / 03$ & 2029 & $1988 / 07$ & 2028 \\
\hline VOGTLE 2 & 4 & $8 / 04$ & 2028 & $89 / 11$ & 2029 & $1989 / 05$ & 2029 \\
\hline WATTS BAR 2 & 4 & $1987 / 06$ & 2027 & $89 / 05$ & 2029 & $1989 / 05$ & 2028 \\
\hline SOUTH TEX UNIT 2 & 6 & $1989 / 04$ & 2028 & $1990 / 10$ & 2030 & $1990 / 04$ & 2030 \\
\hline LIMERICK 2 & 3 & $1990 / 01$ & 2029 & & & $1992 / 08$ & 2032 \\
\hline PERRY 2 & 5 & $1990 / 04$ & 2030 & & & $1992 / 12$ & 2032 \\
\hline WNP - 3 & 10 & & & & & $1994 / 12$ & 2034 \\
\hline BELLEFONTE 1 & 4 & $1989 / 06$ & 2028 & $1996 / 06$ & 2036 & $1995 / 09$ & 2035 \\
\hline MIDLANO-2 & 5 & & & & & $1996 / 06$ & 2036 \\
\hline
\end{tabular}


TABLE A.1. Startup and Shutdown Dates with Federal Energy Region Shown for Utility, No New Orders, Upper Reference and Lower Reference Cases (cont'd)

\begin{tabular}{|c|c|c|c|c|c|c|c|}
\hline \multirow[b]{2}{*}{ Reactor } & \multirow{2}{*}{$\begin{array}{l}\text { Fed. En. } \\
\text { Region }\end{array}$} & \multirow{2}{*}{$\frac{\text { Utility }}{\text { SU }}$} & \multirow{2}{*}{$\begin{array}{c}\text { Case } \\
\text { SD }\end{array}$} & $\begin{array}{r}\text { No N } \\
\text { Orders } \\
\text { Low } \\
\text { Ref. C }\end{array}$ & $\begin{array}{l}\text { w } \\
\text { and } \\
r \\
\text { ses }\end{array}$ & \multicolumn{2}{|c|}{$\begin{array}{c}\text { Upper } \\
\text { Ref. Case } \\
\end{array}$} \\
\hline & & & & SU & SD & SU & $\mathrm{SD}$ \\
\hline BELLEFONTE 2 & 4 & $1990 / 06$ & 2030 & $2001 / 06$ & 2041 & $1998 / 06$ & 2038 \\
\hline GRAND GULF 2 & 4 & & & & & $2001 / 06$ & 2041 \\
\hline SEABROOK 2 & 1 & & & & & $2001 / 06$ & 2041 \\
\hline WNP - 1 & 10 & & & & & $2001 / 06$ & 2042 \\
\hline MIDLAND-1 & 5 & & & & & $2003 / 06$ & 2043 \\
\hline
\end{tabular}


TABLE A.2. Startup and Shutdown Dates of Upper and Lower Reference Case Generic Reactors with Federal Energy Region Assignments

\begin{tabular}{|c|c|c|c|}
\hline \multirow{2}{*}{$\begin{array}{l}\text { Reactor } \\
\text { Name } \\
\end{array}$} & \multirow{2}{*}{$\begin{array}{l}\text { Federal } \\
\text { Energy } \\
\text { Region }\end{array}$} & \multicolumn{2}{|c|}{$\begin{array}{c}\text { Lower Reference } \\
\text { Case BWRs } \\
\end{array}$} \\
\hline & & Startup & Shutdown \\
\hline B060111 & 5 & $2006 / 11$ & 2046 \\
\hline B080107 & 5 & $2008 / 07$ & 2048 \\
\hline B10010i & 2 & $2010 / 01$ & 2050 \\
\hline B100207 & 1 & $2010 / 07$ & 2050 \\
\hline B110105 & 4 & $2011 / 05$ & 2051 \\
\hline B120101 & 7 & $2012 / 01$ & 2052 \\
\hline B120211 & 2 & $2012 / 11$ & 2052 \\
\hline B130101 & 5 & $2013 / 01$ & 2053 \\
\hline B130207 & 5 & $2013 / 07$ & 2053 \\
\hline B140101 & 2 & $2014 / 01$ & 2054 \\
\hline B140201 & 4 & $2014 / 01$ & 2054 \\
\hline 8140301 & 4 & $2014 / 01$ & 2054 \\
\hline 8140411 & 6 & $2014 / 11$ & 2054 \\
\hline B150101 & 5 & $2015 / 01$ & 2055 \\
\hline 8150205 & 7 & $2015 / 05$ & 2055 \\
\hline B160101 & 5 & $2016 / 01$ & 2056 \\
\hline B160212 & 3 & $2016 / 12$ & 2056 \\
\hline B170101 & 1 & $2017 / 01$ & 2057 \\
\hline R180101 & 5 & $2018 / 01$ & 2058 \\
\hline B180211 & 3 & $2018 / 11$ & 2058 \\
\hline B200106 & 4 & $2020 / 06$ & 2060 \\
\hline
\end{tabular}

\begin{tabular}{|c|c|c|c|}
\hline \multirow{2}{*}{$\begin{array}{l}\text { Reactor } \\
\text { Name }\end{array}$} & \multirow{2}{*}{$\begin{array}{l}\text { Federal } \\
\text { Energy } \\
\text { Region }\end{array}$} & \multicolumn{2}{|c|}{$\begin{array}{c}\text { Lower Reference } \\
\text { Case PWRs }\end{array}$} \\
\hline & & Startup & Shutdown \\
\hline P060108 & 4 & $2006 / 08$ & 2046 \\
\hline P070107 & 4 & $2007 / 07$ & 2047 \\
\hline P080103 & 3 & $2008 / 03$ & 2048 \\
\hline P090101 & 9 & $2009 / 01$ & 2049 \\
\hline P090212 & 4 & $2009 / 12$ & 2049 \\
\hline P100101 & 4 & $2010 / 01$ & 2050 \\
\hline P100203 & 6 & $2010 / 03$ & 2050 \\
\hline P110101 & 5 & $2011 / 01$ & 2051 \\
\hline P110201 & 3 & $2011 / 01$ & 2051 \\
\hline P110308 & 5 & $2011 / 08$ & 2051 \\
\hline P120101 & 9 & $2012 / 01$ & 2052 \\
\hline P120201 & 4 & $2012 / 01$ & 2052 \\
\hline P120306 & 3 & $2012 / 06$ & 2052 \\
\hline P130101 & 4 & $2013 / 01$ & 2053 \\
\hline P130201 & 6 & $2013 / 01$ & 2053 \\
\hline P130301 & 4 & $2013 / 01$ & 2053 \\
\hline
\end{tabular}


IABLE A.2. Startup and Shutdown Dates of Upper and Lower Reference Case Generic Reactors with Federal Energy Region Assignments (contd)

\begin{tabular}{|c|c|c|c|}
\hline \multirow{2}{*}{$\begin{array}{l}\text { React or } \\
\text { Name }\end{array}$} & \multirow{2}{*}{$\begin{array}{l}\text { Federal } \\
\text { Energy } \\
\text { Region }\end{array}$} & \multicolumn{2}{|c|}{$\begin{array}{c}\text { Lower Reference } \\
\text { Case PWRs }\end{array}$} \\
\hline & & Startup & Shutdown \\
\hline P130402 & 1 & $2013 / 02$ & 2053 \\
\hline P140101 & 3 & $2014 / 01$ & 2054 \\
\hline P140201 & 9 & $2014 / 01$ & 2054 \\
\hline P140301 & 5 & $2014 / 01$ & 2054 \\
\hline P140401 & 10 & $2014 / 01$ & 2054 \\
\hline P140501 & 5 & $2014 / 01$ & 2054 \\
\hline P140601 & 4 & $2014 / 01$ & 2054 \\
\hline P140701 & 3 & $2014 / 01$ & 2054 \\
\hline P140807 & 9 & $2014 / 07$ & 2054 \\
\hline P150101 & 1 & $2015 / 01$ & 2055 \\
\hline P150201 & 4 & $2015 / 01$ & 2055 \\
\hline P150301 & 3 & $2015 / 01$ & 2055 \\
\hline P150401 & 4 & $2015 / 01$ & 2055 \\
\hline P150508 & 2 & $2015 / 08$ & 2055 \\
\hline P160101 & 4 & $2016 / 01$ & 2056 \\
\hline P160201 & 9 & $2016 / 01$ & 2056 \\
\hline P160312 & 4 & $2016 / 12$ & 2056 \\
\hline P170101 & 6 & $2017 / 01$ & 2057 \\
\hline$P 170201$ & 5 & $2017 / 01$ & 2057 \\
\hline P170301 & 4 & $2017 / 01$ & 2057 \\
\hline P180101 & 2 & $2018 / 01$ & 2058 \\
\hline P180201 & 4 & $2018 / 01$ & 2058 \\
\hline P180308 & 9 & $2018 / 08$ & 2058 \\
\hline P190107 & 4 & $2019 / 07$ & 2059 \\
\hline P200101 & 5 & $2020 / 01$ & 2060 \\
\hline
\end{tabular}


TABLE A.2. Startup and Shutdown Dates of Upper and Lower Reference Case Generic Reactors with Federal Energy Region Assignments (contd)

\begin{tabular}{|c|c|c|c|}
\hline \multirow{2}{*}{$\begin{array}{c}\text { Reactor } \\
\text { Name }\end{array}$} & \multirow{2}{*}{$\begin{array}{l}\text { Federal } \\
\text { Energy } \\
\text { Region }\end{array}$} & \multicolumn{2}{|c|}{$\begin{array}{c}\text { Upper Reference } \\
\text { Case BWRs }\end{array}$} \\
\hline & & Startup & Shutdown \\
\hline 8010104 & 5 & $2001 / 10$ & 2041 \\
\hline $\mathrm{B} 020101$ & 5 & $2002 / 07$ & 2042 \\
\hline B020207 & 2 & $2003 / 01$ & 2043 \\
\hline B030101 & 1 & $2003 / 07$ & 2043 \\
\hline B040101 & 9 & $2004 / 07$ & 2044 \\
\hline B040204 & 4 & $2004 / 10$ & 2044 \\
\hline B050101 & 6 & $2005 / 07$ & 2045 \\
\hline B050206 & 4 & $2005 / 12$ & 2045 \\
\hline B060101 & 9 & $2006 / 07$ & 2046 \\
\hline B060212 & 4 & $2007 / 05$ & 2047 \\
\hline B070102 & 5 & $2007 / 08$ & 2047 \\
\hline B080101 & 5 & $2008 / 07$ & 2048 \\
\hline B080203 & 3 & $2008 / 09$ & 2048 \\
\hline B090101 & 4 & $2009 / 07$ & 2049 \\
\hline 8090204 & 4 & $2009 / 10$ & 2049 \\
\hline B 100101 & 5 & $2010 / 07$ & 2050 \\
\hline B100201 & 3 & $2010 / 07$ & 2050 \\
\hline 8100311 & 3 & $2011 / 05$ & 2051 \\
\hline Bl 10101 & 9 & $2011 / 07$ & 2051 \\
\hline B 110204 & 4 & $2011 / 10$ & 2051 \\
\hline B120101 & 4 & $2012 / 07$ & 2052 \\
\hline B 120201 & 5 & $2012 / 07$ & 2052 \\
\hline B120306 & 4 & $2012 / 12$ & 2052 \\
\hline B130101 & 3 & $2013 / 07$ & 2053 \\
\hline B130201 & 4 & $2013 / 07$ & 2053 \\
\hline B130302 & 2 & $2013 / 08$ & 2053 \\
\hline B140101 & 1 & $2014 / 07$ & 2054 \\
\hline B 140201 & 3 & $2014 / 07$ & 2054 \\
\hline B140301 & 4 & $2014 / 07$ & 2054 \\
\hline B140401 & 5 & $2014 / 07$ & 2054 \\
\hline B140503 & 6 & $2014 / 09$ & 2054 \\
\hline B150101 & 4 & $2015 / 07$ & 2055 \\
\hline B150201 & 4 & $2015 / 07$ & 2055 \\
\hline B150301 & 2 & $2015 / 07$ & 2055 \\
\hline B150406 & 6 & $2015 / 12$ & 2055 \\
\hline$B 160101$ & 10 & $2016 / 07$ & 2056 \\
\hline B 160201 & 4 & $2016 / 07$ & 2056 \\
\hline B160311 & 4 & $2017 / 05$ & 2057 \\
\hline B170101 & 5 & $2017 / 07$ & 2057 \\
\hline B 170201 & 4 & $2017 / 07$ & 2057 \\
\hline B170308 & 4 & $2018 / 02$ & 2058 \\
\hline B180101 & 5 & $2018 / 07$ & 2058 \\
\hline
\end{tabular}


TABLE A.2. Startup and Shutdown Dates of Upper and Lower Reference Case Generic Reactors with Federal Energy Region Assignments (contd)

\begin{tabular}{|c|c|c|c|}
\hline Reactor Name & $\begin{array}{l}\text { Federal Energy } \\
\text { Region } \\
\end{array}$ & $\begin{array}{l}\text { Upper Reference } \\
\text { Startup }\end{array}$ & $\begin{array}{l}\text { Case BWRs } \\
\text { Shutdown }\end{array}$ \\
\hline $\begin{array}{l}\text { B180204 } \\
\text { B190101 } \\
\text { B190207 } \\
\text { B200101 } \\
8200206\end{array}$ & $\begin{array}{l}3 \\
6 \\
4 \\
4 \\
4\end{array}$ & $\begin{array}{l}2018 / 10 \\
2019 / 07 \\
2020 / 01 \\
2020 / 07 \\
2020 / 12\end{array}$ & $\begin{array}{l}2058 \\
2059 \\
2060 \\
2060 \\
2060\end{array}$ \\
\hline
\end{tabular}

\begin{tabular}{|c|c|}
\hline Reactor Name & Region \\
\hline P010101 & 4 \\
\hline P010208 & 4 \\
\hline P020101 & 9 \\
\hline P020201 & 3 \\
\hline P020303 & 4 \\
\hline P030101 & 4 \\
\hline P030201 & 5 \\
\hline P030301 & 6 \\
\hline P040101 & 3 \\
\hline P040201 & 4 \\
\hline P040301 & 5 \\
\hline P040406 & 2 \\
\hline P050101 & 5 \\
\hline P050201 & 3 \\
\hline P050301 & 4 \\
\hline P060101 & 1 \\
\hline P060201 & 5 \\
\hline P060301 & 7 \\
\hline P060408 & 3 \\
\hline P070101 & 4 \\
\hline P070201 & 2 \\
\hline P070304 & 10 \\
\hline P080101 & 6 \\
\hline P080201 & 4 \\
\hline P080301 & 9 \\
\hline P080405 & 4 \\
\hline P090101 & 2 \\
\hline P090201 & 5 \\
\hline P090301 & 1 \\
\hline P090408 & 5 \\
\hline P100101 & 4 \\
\hline P100201 & 6 \\
\hline
\end{tabular}

Upper Reference Case PWRs Startup Shutdown

$2001 / 07$

$2002 / 02 \quad 2042$

$2002 / 07 \quad 2042$

$2002 / 07 \quad 2042$

$2002 / 09 \quad 2042$

$2003 / 07 \quad 2043$

$2003 / 07 \quad 2043$

$2003 / 07 \quad 2043$

$2004 / 07 \quad 2044$

$2004 / 07 \quad 2044$

$2004 / 07 \quad 2044$

$2004 / 12 \quad 2044$

$2005 / 07 \quad 2045$

$2005 / 07 \quad 2045$

$2005 / 07 \quad 2045$

$2006 / 07 \quad 2046$

$2006 / 07 \quad 2046$

$2006 / 07 \quad 2046$

$2007 / 02 \quad 2047$

$2007 / 07 \quad 2047$

$2007 / 07 \quad 2047$

$2007 / 10 \quad 2047$

$2008 / 07 \quad 2048$

$2008 / 07 \quad 2048$

$2008 / 07 \quad 2048$

$2008 / 11 \quad 2048$

$2009 / 07 \quad 2049$

$2009 / 07 \quad 2049$

$2009 / 07 \quad 2049$

$2010 / 02 \quad 2050$

$2010 / 07 \quad 2050$

$2010 / 07 \quad 2050$ 
TABLE A.2. Startup and Shutdown Dates of Upper and Lower Reference Case Generic Reactors with Federal Energy Region Assignments (contd)

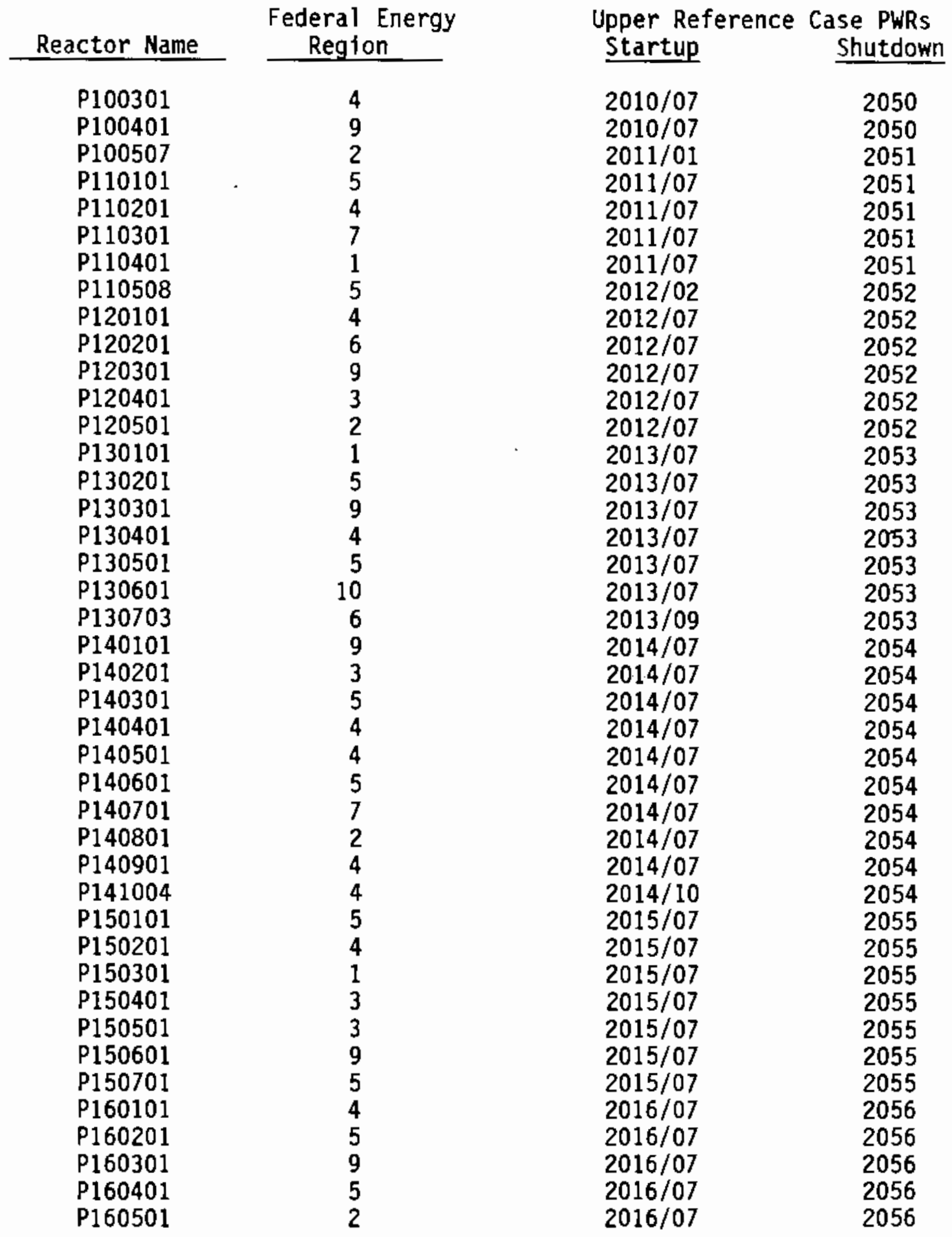


TABLE A.2. Startup and Shutdown Dates of Upper and Lower Reference Case Generic Reactors with Federal Energy Region Assignments (contd)

\begin{tabular}{ccc} 
Reactor Name & & $\begin{array}{c}\text { Federal Energy } \\
\text { Region }\end{array}$ \\
\cline { 1 - 1 } P160608 & & 3 \\
P170101 & & 5 \\
P170201 & & 7 \\
P170301 & & 1 \\
P170401 & & 6 \\
P170501 & & 9 \\
P170605 & 3 \\
P180101 & 4 \\
P180201 & 9 \\
P180301 & & 2 \\
P180401 & & 4 \\
P180508 & 5 \\
P190101 & 4 \\
P190201 & 1 \\
P190303 & 2 \\
P200101 & 5 \\
P200201 & 9 \\
P200301 & 3 \\
P200402 & 5
\end{tabular}

\begin{tabular}{lc}
$\begin{array}{c}\text { Upper Reference } \\
\text { Startup }\end{array}$ & $\begin{array}{c}\text { Case PWRs } \\
\text { Shutdown }\end{array}$ \\
\cline { 2 - 2 } $2017 / 02$ & 2057 \\
$2017 / 07$ & 2057 \\
$2017 / 07$ & 2057 \\
$2017 / 07$ & 2057 \\
$2017 / 07$ & 2057 \\
$2017 / 07$ & 2057 \\
$2017 / 11$ & 2057 \\
$2018 / 07$ & 2058 \\
$2018 / 07$ & 2058 \\
$2018 / 07$ & 2058 \\
$2018 / 07$ & 2058 \\
$2019 / 02$ & 2059 \\
$2019 / 07$ & 2059 \\
$2019 / 07$ & 2059 \\
$2019 / 09$ & 2059 \\
$2020 / 07$ & 2060 \\
$2020 / 07$ & 2060 \\
$2020 / 07$ & 2060 \\
$2020 / 08$ & 2060
\end{tabular}


TABLE A.3. Upper Reference Case, 1985 Inventory and Projected Annual Reactor Discharges

\begin{tabular}{|c|c|c|c|c|c|c|c|c|c|c|c|c|c|c|c|c|c|c|c|c|c|c|}
\hline & Iny & & & & & ASSENB & DIES & & & & & Inv & & & & & NTIHN & & & & & \\
\hline REACTQR & $\underline{1985}$ & 1986 & 1987 & 1988 & $\underline{1989}$ & 1998 & $\underline{1891}$ & 1992 & $\underline{1993}$ & 1994 & 1995 & $\underline{1985}$ & 1980 & 1987 & $\underline{1988}$ & $\underline{1989}$ & 19901 & 1991 & 1992 & $\underline{1993}$ & 1994 & $\underline{1995}$ \\
\hline ARK NUCLEAR 1 & 378 & 59 & 6 & 52 & $\theta$ & 52 & 54 & 0 & 47 & $B$ & 49 & 175 & 27 & $\theta$ & 24 & a & 24 & 25 & 6 & 22 & 0 & 23 \\
\hline ARK NUCLEAR 2 & 228 & 54 & 0 & 55 & 58 & 6 & 65 & 9 & 56 & $\theta$ & 69 & 95 & 23 & છ & 23 & 24 & $\theta$ & 27 & 0 & 23 & $\theta$ & 25 \\
\hline 日 VALEY 1 & 218 & 63 & 56 & - & 62 & 81 & 0 & 65 & 81 & 61 & 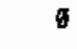 & 100 & 29 & 28 & 9 & 29 & 28 & $\theta$ & 36 & 28 & 28 & $\theta$ \\
\hline B YAUEY 2 & $\bullet$ & o & 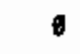 & $\theta$ & $\theta$ & 25 & 54 & 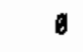 & 58 & 54 & 0 & a & 6 & 6 & ฮ & 0 & 12 & 25 & 6 & 26 & 25 & 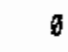 \\
\hline B日—EONTE I & 5 & 6 & 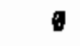 & $\theta$ & 0 & 0 & 6 & a & 0 & 6 & 6 & 0 & 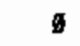 & 0 & 0 & $\theta$ & 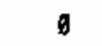 & $\theta$ & $a$ & 0 & $\theta$ & b \\
\hline BELEONTE 2 & 6 & 6 & 6 & $\theta$ & $\theta$ & - & 6 & $\theta$ & 6 & 6 & 0 & 5 & 0 & $\theta$ & $\theta$ & 0 & 8 & 6 & $\theta$ & $\theta$ & 6 & 8 \\
\hline BIG ROCK 1 & 192 & 18 & 6 & 14 & 17 & 17 & 19 & 17 & 16 & 6 & 18 & 25 & 2 & $\theta$ & 2 & 2 & 2 & 2 & 2 & 2 & 0 & 2 \\
\hline BRAIDWOOD 1 & - & $\theta$ & 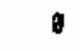 & $\theta$ & 114 & 0 & 63 & 78 & 0 & 61 & 83 & 6 & 6 & 6 & 8 & 48 & 0 & 27 & 33 & $\theta$ & 26 & 35 \\
\hline BRAIDHOCD 2 & 6 & $\theta$ & 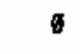 & 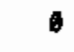 & $\theta$ & - & 73 & 73 & 6 & 61 & $8 B$ & 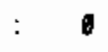 & 6 & $\theta$ & 9 & $\theta$ & 8 & 31 & 31 & 6 & 26 & 36 \\
\hline GROTNS FERRY1 & 1328 & 9 & 0 & $\theta$ & $\theta$ & 0 & 0 & 293 & 6 & 189 & 6 & 246 & 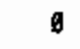 & 0 & $\theta$ & 6 & 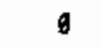 & $\theta$ & 37 & 8 & 34 & $\theta$ \\
\hline BROWNS FERRY2 & 1192 & 0 & 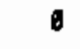 & 245 & 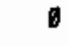 & t & 211 & 199 & 9 & 190 & $\theta$ & 221 & 6 & $a$ & 45 & 0 & 0 & $3 a$ & 36 & $b$ & 35 & $\theta$ \\
\hline BROWNS FERRY3 & 1064 & 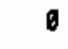 & 0 & • & 229 & 0 & 218 & 8 & 190 & 192 & 6 & 185 & 6 & 6 & 8 & 42 & $\theta$ & 40 & 9 & 35 & 35 & $b$ \\
\hline BRUNSWICK 1 & 840 & 0 & 149 & 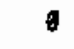 & 153 & 156 & - & 183 & - & 155 & 183 & 156 & 8 & 27 & 0 & 29 & 29 & 0 & 30 & 8 & 29 & 38 \\
\hline BRUNSWICK 2 & 758 & b & 6 & 168 & 157 & 0 & 176 & ๑ & 154 & 155 & $\theta$ & 141 & 9 & $\theta$ & 30 & 29 & 8 & 33 & $\theta$ & 29 & 29 & $g$ \\
\hline QYRRON 1 & 6 & 8 & 88 & 75 & 6 & 75 & $B 4$ & 0 & 76 & 70 & 6 & 6 & $\theta$ & 29 & 32 & $\theta$ & 32 & 36 & 0 & 30 & 30 & 0 \\
\hline GYRON 2 & $\theta$ & $\theta$ & $\theta$ & 0 & 114 & 59 & $\theta$ & 78 & 88 & $\theta$ & 82 & 6 & 0 & $\theta$ & 0 & 48 & 25 & $\theta$ & 33 & 29 & 0 & 35 \\
\hline CALLAIIAY 1 & छ & 77 & 74 & 6 & 82 & 74 & - & 85 & 73 & $\theta$ & 85 & 6 & 35 & 34 & $\theta$ & 35 & 31 & 6 & 36 & 31 & 0 & 36 \\
\hline CALYERT CLF 1 & 542 & 71 & 0 & 78 & a & 78 & $\theta$ & 82 & 0 & 77 & 6 & 208 & 27 & $\theta$ & 29 & $\theta$ & 37 & 0 & 31 & $\theta$ & 29 & $\theta$ \\
\hline CALYERT CLF 2 & 468 & 0 & 68 & - & 75 & 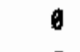 & 98 & 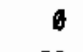 & 77 & b & 81 & 179 & 6 & 25 & 8 & 28 & $\theta$ & 36 & 6 & 29 & $\theta$ & 38 \\
\hline CATANEA 1 & 6 & 59 & 53 & 56 & 54 & $\theta$ & 61 & 58 & 53 & 6 & 56 & $\theta$ & 25 & 22 & 24 & 23 & 8 & 28 & 24 & 22 & $\theta$ & 24 \\
\hline CATAMBA 2 & $\bullet$ & v & $\theta$ & 90 & 86 & 50 & $\theta$ & 57 & 49 & 48 & $\theta$ & $\theta$ & $\theta$ & 0 & 38 & 34 & 21 & $\theta$ & 24 & 21 & 20 & $\theta$ \\
\hline CLINTON 1 & 6 & 6 & 6 & 273 & 272 & 0 & 151 & 193 & 0 & 137 & 188 & 6 & 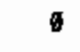 & 0 & 56 & 56 & 6 & 28 & 35 & 0 & 25 & 34 \\
\hline COMANCHE PK 1 & $\theta$ & 6 & 6 & 88 & 75 & 49 & 51 & 54 & 49 & 56 & 52 & $\theta$ & 8 & 0 & 41 & 35 & 23 & 23 & 22 & 20 & 20 & 21 \\
\hline COMANCHE PK 2 & 6 & 6 & 6 & 6 & $\theta$ & 48 & 52 & 57 & 51 & 45 & 50 & - & 6 & $\theta$ & 0 & 6 & 19 & 22 & 24 & 22 & 18 & 23 \\
\hline $\operatorname{cook} 1$ & 548 & 6 & 59 & 67 & $\theta$ & 68 & 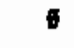 & 71 & 87 & 8 & 70 & 238 & $\theta$ & 26 & 31 & 0 & 31 & $\theta$ & 33 & 31 & 6 & 32 \\
\hline $\cos 2$ & 335 & 86 & 88 & 6 & 75 & - & 84 & 78 & b & 73 & 78 & 154 & 34 & 27 & 0 & 30 & 0 & 34 & 32 & $\theta$ & 29 & 32 \\
\hline CDOPER STN & 874 & 137 & 6 & 115 & 95 & 98 & 112 & 162 & 100 & 94 & 161 & 164 & 25 & 6 & 21 & 17 & 18 & $2 \theta$ & 19 & 18 & 17 & 18 \\
\hline CRYSTAL RVR 3 & 386 & 6 & 69 & 6 & 85 & 0 & 69 & ๑ & 60 & 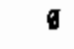 & 83 & 142 & $\theta$ & 32 & 0 & 38 & 0 & 32 & 0 & 28 & 0 & 29 \\
\hline DAYIS-BESSE 1 & 197 & 8 & 49 & 52 & $\theta$ & 52 & 59 & - & 51 & 51 & 0 & 93 & 0 & 23 & 24 & $\theta$ & 24 & 28 & $\theta$ & 24 & 24 & $\theta$ \\
\hline DIAQLD CANYON-1 & 6 & 63 & 6 & 76 & 88 & $\theta$ & 104 & 0 & 87 & 9 & 95 & 10 & 29 & 0 & 32 & 46 & 0 & 48 & $\theta$ & 40 & $\theta$ & 44 \\
\hline DIAQLD CANYON-2 & 6 & 6 & 53 & 0 & 68 & 88 & 6 & 95 & b & 87 & - & $\theta$ & 6 & 24 & $\theta$ & 31 & 40 & $\theta$ & 44 & 0 & 40 & $\theta$ \\
\hline DRESOEN I & 889 & $\theta$ & 0 & 6 & - & $\theta$ & 6 & $\theta$ & 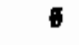 & 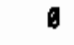 & 6 & 78 & 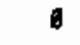 & 6 & 0 & 6 & $\theta$ & 0 & 0 & $\theta$ & 0 & $\theta$ \\
\hline DRESDEY 2 & 1414 & 177 & 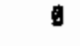 & 144 & 135 & $\theta$ & 151 & 140 & 6 & 132 & 139 & 257 & 31 & 0 & 24 & 23 & 0 & 25 & 23 & 6 & 22 & 23 \\
\hline DRESDEY 3 & 1458 & $\theta$ & 116 & 9 & 136 & 134 & g & 140 & 132 & 0 & 139 & 286 & 6 & 19 & $\theta$ & 23 & 22 & $\theta$ & 23 & 22 & 6 & 23 \\
\hline DUANE ARNOLD & 898 & $\boldsymbol{b}$ & 99 & 164 & 6 & 102 & 122 & 0 & 99 & 160 & 6 & 129 & 0 & 18 & 19 & 0 & 19 & 22 & $\theta$ & 18 & 18 & $\theta$ \\
\hline ENRICO FERHI-2 & 6 & 6 & 9 & 281 & 249 & $\theta$ & 287 & - & 247 & 6 & 257 & 6 & 0 & 0 & 37 & 48 & 6 & 49 & 0 & 45 & 0 & 47 \\
\hline FARLEY 1 & 349 & 59 & 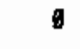 & 80 & 58 & 0 & 69 & 81 & 6 & 80 & 81 & 160 & 27 & 6 & 28 & 27 & 6 & 32 & 28 & $\theta$ & 28 & 28 \\
\hline FARLEY 2 & 188 & 83 & 53 & 0 & 81 & 58 & $\theta$ & 83 & 57 & 6 & 83 & 88 & 29 & 24 & $\theta$ & 28 & $27 \mathrm{~J}$ & 8 & 29 & 26 & $\theta$ & 29 \\
\hline FITZPATRICK & 1012 & $b$ & 144 & 141 & $\theta$ & 151 & 173 & 0 & 147 & 147 & 6 & 189 & a & 28 & $2 B$ & 6 & 28 & 31 & $\sigma$ & 26 & 26 & $\theta$ \\
\hline FORT CALHOUN & 347 & $B$ & 34 & 38 & $\theta$ & 37 & 39 & 0 & 38 & 31 & B & 128 & $\theta$ & 12 & 13 & 8 & 13 & 14 & $\theta$ & 13 & 11 & $\theta$ \\
\hline GINNA & 386 & 36 & 27 & 27 & 27 & 27 & 30 & 28 & 27 & 27 & $2 B$ & 139 & 11 & 10 & 10 & 9 & 9 & 16 & 9 & 9 & 9 & 9 \\
\hline GRAND GUF 1 & 8 & 244 & 203 & 0 & 211 & 224 & $\theta$ & 235 & 221 & $\theta$ & 234 & g & 45 & 37 & 6 & 38 & 48 & $\sigma$ & 41 & 39 & $\theta$ & 41 \\
\hline GRAND GUF 2 & 0 & 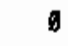 & 0 & 0 & $\theta$ & 6 & 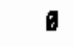 & 6 & 0 & 8 & g & 6 & 0 & 0 & 6 & g & 0 & g & 6 & $\theta$ & $\theta$ & $\theta$ \\
\hline HADDAN NECK & 544 & 48 & 41 & 41 & $\theta$ & 41 & 51 & 47 & 6 & 44 & 50 & 224 & 28 & $\$ 7$ & 17 & 0 & 17 & 21 & 19 & $\theta$ & 18 & 21 \\
\hline HARRIS 1 & 6 & 6 & 0 & 79 & B7 & 0 & 43 & 44 & 8 & 36 & 43 & 6 & 0 & $\theta$ & 37 & 31 & $\theta$ & 26 & 20 & $\theta$ & 17 & 20 \\
\hline HATCH 1 & 1188 & $\sigma$ & 141 & 157 & $\theta$ & 154 & 177 & 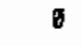 & 154 & 155 & $\theta$ & 218 & 0 & 28 & 29 & 8 & 28 & 33 & $b$ & 29 & 29 & $b$ \\
\hline HATCH 2 & 548 & 175 & 139 & 1 & 155 & 155 & $\theta$ & 163 & 153 & $\theta$ & 162 & 100 & 32 & 25 & $\theta$ & 29 & 29 & $\theta$ & 30 & 28 & $\theta$ & 30 \\
\hline HOPE CREEK & 0 & 0 & 0 & $\theta$ & 324 & 186 & 5 & 237 & 199 & $\theta$ & 232 & $\theta$ & 0 & $\theta$ & $\theta$ & 60 & 33 & $\theta$ & 44 & 36 & $\theta$ & 42 \\
\hline MEOLDT BAY & 390 & $\theta$ & 0 & 6 & 0 & 0 & 0 & $\theta$ & $\theta$ & $\theta$ & 0 & 29 & 0 & 0 & 6 & 6 & 6 & 8 & $\theta$ & 0 & 0 & \\
\hline
\end{tabular}


TABLE A.3. Upper Reference Case, 1985 Inventory and Projected Annual Reactor Discharges (contd)

REACTOR

INDIAN PT 1

INDIAN PT 2

INDIAN PT 3

KEXNUNEE

LACROSSE

LASALE CTY 1

LSNAL CTY 2

LIMERICK 1

LIMERICK 2

MAINE YANKEE

MCQUIRE 1

MCOJIRE 2

NIDLAND-1

VIDLAND-2

WIUSTONE 1

MILLSTONE 2

MILLSTONE 3

NONTICEIS

NINE NILE PT1

WIME UILE PT2

NORTH ANNA 1

NORTH ANNA 2

DCONEE 1

OCONEE 2

OCONEE 3

QYSTER CRK 1

PALISADES

PALO VEROE 1

PALO VERDE 2

PALO YERDE 3

PEACHBOTTOY 2

PEACHBOTTOM 3

PERRY 1

PERRY 2

PILGRIM 1

PQINT BEACH 1

POINT BEACH 2

PRUIRIE ISL 1

PRAIRIE ISL 2

QUAD CITIES 1

QUAD CITIES 2

RANCHO SECO-1

ROBINSON 2

RVR BEND 1

SALEN UNIT 2

SALE 1

SAN DNOFRE 1

SAN ONOFRE 2

SAN DNOFRE 3
InY _ASSEIRLIES

UTIHU

$\underline{1985} 198819871989199919901991$

Iny

$1992199319941995 \quad 1985198619871988198919961991199219931994 \underline{1995}$

$\begin{array}{lllllllllllllllllllllll}398 & 62 & 52 & 0 & 57 & 57 & 6 & 61 & 0 & 57 & 80 & 179 & 28 & 24 & 6 & 28 & 26 & 0 & 28 & 6 & 26 & 27\end{array}$

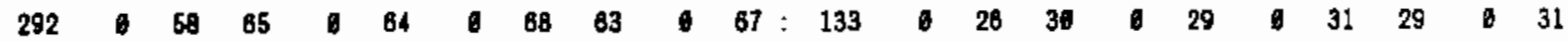

$\begin{array}{lllllllllllllllllllllll}352 & 25 & 37 & 41 & 33 & 31 & 36 & 33 & 31 & 31 & 33 & 138 & 9 & 14 & 18 & 13 & 12 & 14 & 13 & 12 & 12 & 13\end{array}$

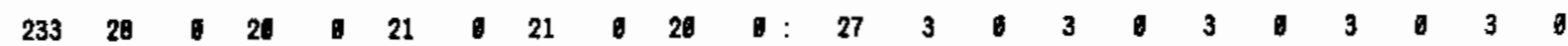

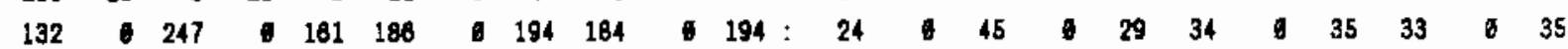

$\begin{array}{llllllllllllllllllllllllll}6 & 297 & 199 & 187 & 212 & 195 & 193 & 194: & 0 & 38 & 0 & 38 & 34 & 0 & 39 & 35 & 6 & 33 & 35\end{array}$

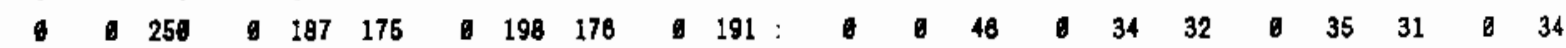

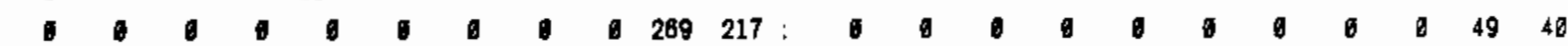

$\begin{array}{llllllllllllllllllllllll}787 & 0 & 56 & 63 & 62 & 0 & 79 & 0 & 61 & 61 & 0 & 297 & 0 & 19 & 23 & 23 & 0 & 27 & 0 & 23 & 23 & 0\end{array}$

$\begin{array}{lllllllllllllllllllllll}135 & 67 & 52 & 57 & 0 & 55 & 61 & 57 & 6 & 53 & 58 & : & 82 & 31 & 22 & 24 & 6 & 23 & 26 & 24 & 0 & 22 & 24\end{array}$

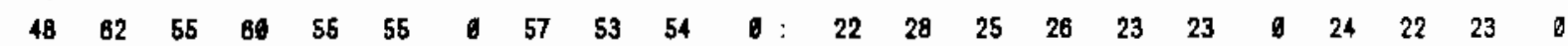

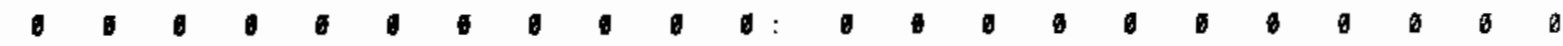

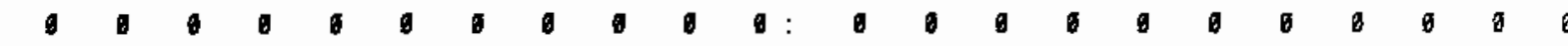

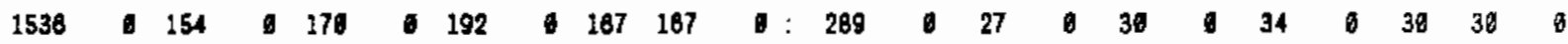

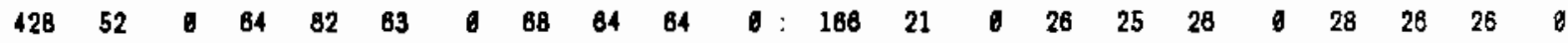

$\begin{array}{llllllllllllllllllllll}6 & 58 & 121 & 85 & 5 & 75 & 64 & 0 & 79 & : & 0 & 6 & 27 & 6 & 58 & 36 & 0 & 35 & 36 & 0 & 36\end{array}$

$\begin{array}{lllllllllllllllllllllll}438 & 124 & 81 & 9 & 92 & 92 & 184 & 98 & 0 & 90 & 95 & 9 & 82 & 22 & 15 & 0 & 18 & 16 & 18 & 17 & 0 & 15 & 16\end{array}$

$\begin{array}{lllllllllllllllllllllll}1244 & 185 & 173 & 162 & 173 & 0 & 147 & 0 & 235 & 33 & 6 & 31 & 6 & 29 & 6 & 36 & 0 & 26 & 9\end{array}$

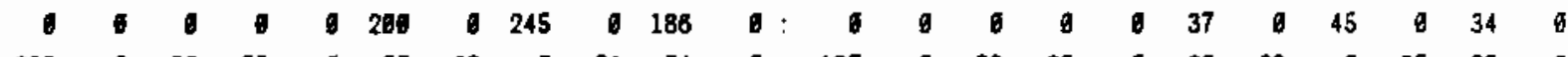

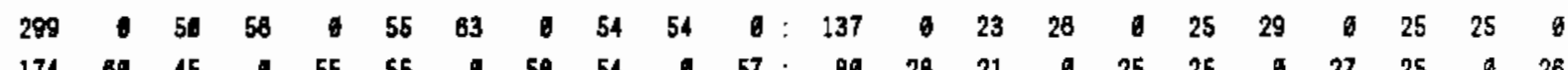

$$
\begin{array}{llllllllllllllllllllllll}
174 & 69 & 45 & 65 & 55 & 5 & 58 & 54 & 0 & 57 & 0 & 80 & 28 & 21 & 0 & 25 & 25 & 0 & 27 & 25 & 0 & 26
\end{array}
$$

$\begin{array}{lllllllllllllllllllllll}578 & 55 & 45 & 50 & 5 & 51 & 58 & 53 & 0 & 56 & 53 & 288 & 25 & 21 & 23 & 0 & 24 & 27 & 25 & 0 & 23 & 25\end{array}$

$\begin{array}{lllllllllllllllllllllll}412 & 55 & 45 & 0 & 51 & 51 & 5 & 53 & 56 & 58 & 0 & : & 191 & 25 & 21 & 0 & 24 & 24 & 0 & 25 & 23 & 23 & 0\end{array}$

$\begin{array}{llllllllllllllllllllllll}388 & 45 & 50 & 51 & 6 & 57 & 53 & 6 & 50 & 53 & 186 & 0 & 21 & 23 & 24 & 0 & 28 & 25 & 6 & 23 & 25\end{array}$

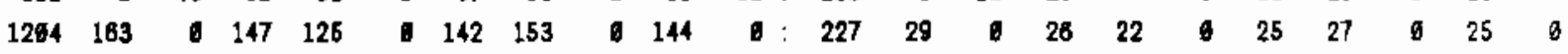

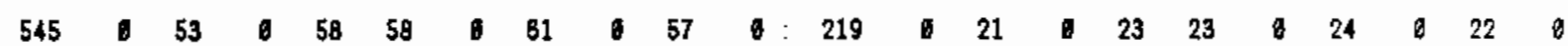

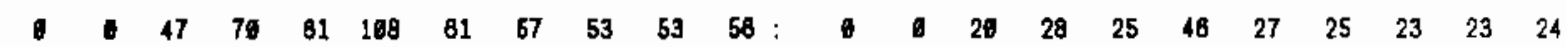

$\begin{array}{llllllllllllllllllllll} & 51 & 128 & 98 & 58 & 48 & 54 & 56 & 48 & 57 & 0 & 0 & 0 & 22 & 51 & 39 & 21 & 21 & 24 & 22 & 21 & 25\end{array}$

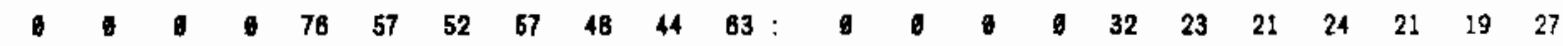

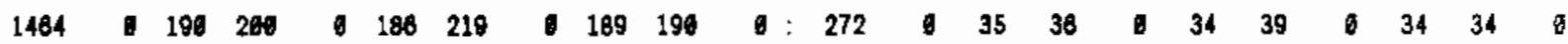

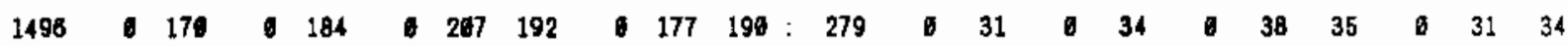

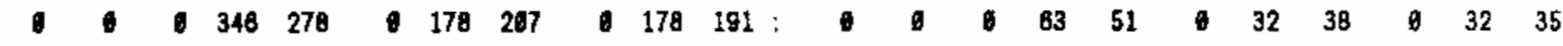

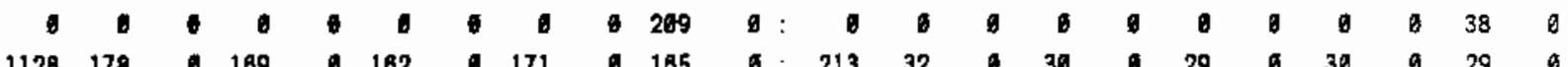

$\begin{array}{lllllllllllllllllllllll}483 & 33 & 32 & 32 & 39 & 28 & 36 & 28 & 27 & 27 & 28 & 161 & 13 & 13 & 13 & 11 & 10 & 11 & 10 & 10 & 10 & 10\end{array}$

$\begin{array}{lllllllllllllllllllllll}384 & 32 & 32 & 32 & 36 & 27 & 31 & 28 & 27 & 27 & 28 & 153 & 13 & 13 & 11 & 11 & 10 & 11 & 10 & 10 & 10 & 10\end{array}$

$\begin{array}{llllllllllllllllllllll}328 & 57 & 31 & 34 & 34 & 34 & 39 & 3 & 33 & 33 & 35 & 128 & 21 & 11 & 12 & 12 & 12 & 14 & 6 & 12 & 12 & 12\end{array}$

$\begin{array}{lllllllllllllllllllllll}374 & 37 & 33 & 33 & 34 & 38 & 36 & 33 & 33 & 35 & 146 & 14 & 9 & 11 & 12 & 12 & 13 & 12 & 12 & 12 & 12\end{array}$

$\begin{array}{llllllllllllllllllllllllll}1269 & 171 & 131 & 0 & 137 & 136 & 142 & 144 & 144 & 8 & 141 & 228 & 36 & 23 & 0 & 24 & 24 & 9 & 25 & 25 & 9 & 25\end{array}$

$\begin{array}{llllllllllllllllllllllllllll}1276 & 141 & 144 & 137 & 155 & 141 & 8 & 134 & 149 & 241 & 25 & 0 & 25 & 24 & 9 & 27 & 25 & 0 & 24 & 25\end{array}$

$\begin{array}{llllllllllllllllllllllll}285 & 53 & 5 & 55 & 59 & 68 & 68 & 54 & 0 & 51 & 54 & 129 & 25 & 6 & 26 & 27 & 6 & 31 & 25 & 0 & 24 & 25\end{array}$

$\begin{array}{lllllllllllllllllllllll}222 & 44 & 38 & 52 & 0 & 48 & 46 & 5 & 51 & 46 & 0 & : & 96 & 19 & 16 & 21 & 6 & 17 & 28 & 0 & 21 & 17 & 9\end{array}$

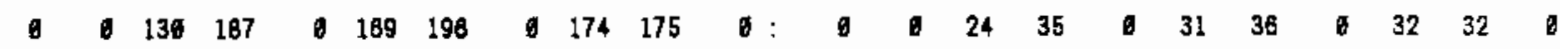

$\begin{array}{llllllllllllllllllllllll}115 & 48 & 0 & 86 & 74 & 5 & 82 & 72 & 9 & 64 & 71 & 53 & 22 & 0 & 37 & 34 & 9 & 38 & 33 & 9 & 29 & 33\end{array}$

$\begin{array}{lllllllllllllllllllllll}270 & 71 & 61 & 6 & 72 & 82 & 9 & 85 & 71 & 6 & 86 & : & 124 & 33 & 28 & 0 & 33 & 28 & 6 & 39 & 33 & 0 & 39\end{array}$

$\begin{array}{lllllllllllllllllllllll}94 & 47 & 6 & 46 & 45 & 0 & 6 & 48 & 17 & 43 & 6 & 35 & 17 & 0 & 17 & 17 & 0 & 9 & 17 & 0 & 18 & 0\end{array}$

$\begin{array}{lllllllllllllllllllllll}68 & 83 & 68 & 0 & 76 & 0 & 86 & 0 & 74 & 6 & 78 & 28 & 33 & 29 & 0 & 32 & 9 & 37 & 9 & 32 & 8 & 33\end{array}$

$\begin{array}{lllllllllllllllllllllll}68 & 81 & 78 & 75 & 75 & 79 & 79 & 5 & 75 & 78 & 28 & 32 & 8 & 32 & 0 & 32 & 0 & 34 & 0 & 32 & 33\end{array}$ 
TABLE A.3. Upper Reference Case, 1985 Inventory and Projected Annual Reactor Discharges (contd)

\begin{tabular}{|c|c|c|c|c|c|c|c|c|c|c|c|c|c|c|c|c|c|c|c|c|c|c|}
\hline \multirow[b]{2}{*}{ REACTOR } & \multirow{2}{*}{$\frac{\text { Iny }}{\underline{1085}}$} & \multicolumn{10}{|c|}{ ASSEURIES } & \multirow{2}{*}{$\frac{\text { Inv }}{1985}$} & \multicolumn{10}{|c|}{ MIIM } \\
\hline & & 1986 & $\underline{1987}$ & 1988 & 1989 & 1990 & 1991 & 1992 & $\underline{1993}$ & 1994 & 1995 & & 1988 & 1907 & 1988 & 1999 & 1998 & 1991 & 1992 & 1993 & 1994 & $\underline{1995}$ \\
\hline SEXEROOK 1 & 1 & 9 & 0 & I & 78 & 44 & 53 & $\theta$ & 49 & 45 & 58 & 6 & 1 & 0 & 0 & 35 & 20 & 24 & t & 23 & 21 & 27 \\
\hline SEABROOK 2 & 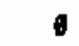 & ] & - & - & 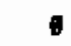 & 6 & $\boldsymbol{t}$ & 6 & 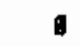 & 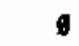 & 6 & 1 & 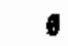 & 0 & 6 & 0 & $\theta$ & 6 & 6 & $\theta$ & $\theta$ & 0 \\
\hline SEqUOYAH 1 & 212 & g & $\boldsymbol{0}$ & 88 & 0 & 88 & D & 71 & 87 & $\theta$ & 71 & 97 & - & 6 & 31 & $\theta$ & 31 & 6 & 33 & 31 & $\theta$ & 33 \\
\hline SEQUOYAH 2 & 138 & 73 & 6 & 69 & 6 & 68 & 6 & 71 & - & B7 & 70 & 62 & 34 & 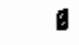 & 32 & a & 31 & 0 & 33 & 8 & 31 & 32 \\
\hline HOREHAN & 6 & 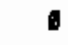 & 0 & 6 & 0 & $\theta$ & 145 & $\theta$ & 142 & 99 & 6 & $\theta$ & 0 & 0 & 0 & 1 & 0 & 27 & $B$ & 28 & 18 & $\theta$ \\
\hline EOUTH TEXAS, UNIT 1 & 6 & - & $\theta$ & 6 & 70 & 42 & 40 & 43 & 46 & 39 & 49 & 5 & 6 & - & - & 38 & 23 & 22 & 23 & 22 & 21 & 27 \\
\hline SOUTH TEUS, UNIT 2 & 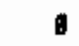 & $\theta$ & 0 & 0 & 0 & 6 & 48 & 48 & 40 & 37 & 55 & 9 & 6 & 6 & $\theta$ & 6 & 0 & 25 & 28 & 22 & 20 & 30 \\
\hline ST LUCIE 1 & 444 & 0 & 55 & 65 & b & 60 & 73 & 6 & 63 & 63 & 6 & 189 & 0 & 21 & 24 & 6 & 23 & 28 & $\varnothing$ & 24 & 24 & $\theta$ \\
\hline ST LUCIE 2 & 80 & 76 & 53 & 6 & 63 & 81 & 6 & 84 & 66 & 6 & 54 & 31 & 28 & 20 & 0 & 24 & 24 & 0 & 25 & 23 & 0 & 25 \\
\hline SLPER 1 & 112 & 日 & 52 & 59 & $\theta$ & 57 & 65 & $\theta$ & 57 & 57 & a & 51 & $\theta$ & 24 & 27 & $\theta$ & 28 & 36 & 0 & 26 & 26 & $\theta$ \\
\hline URRY 1 & 474 & 47 & $\theta$ & 45 & 45 & 44 & 0 & 47 & 43 & 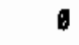 & 47 & 215 & 21 & - & 21 & 21 & 20 & 0 & 22 & 29 & $\theta$ & 22 \\
\hline SURRY 2 & 362 & 52 & 0 & 37 & 48 & $\theta$ & 51 & 47 & 0 & 44 & 47 & 184 & 24 & 6 & 17 & 22 & 0 & 23 & 22 & 9 & 29 & 22 \\
\hline SLSQUEHANKA 1 & 192 & 272 & 163 & $\theta$ & 204 & 187 & - & 199 & 188 & 6 & 198 & 35 & 50 & 34 & 0 & 36 & 33 & 0 & 35 & 32 & 0 & 34 \\
\hline JUSQUEHANNA 2 & $\theta$ & 6 & 248 & 181 & 0 & 188 & 216 & 0 & 188 & 187 & - & $\forall$ & $\theta$ & 46 & 33 & a & 34 & 37 & $B$ & 32 & 32 & $\theta$ \\
\hline 3 UILE ISL 1 & 209 & $\theta$ & 58 & 67 & - & 61 & 86 & 6 & 81 & 81 & 6 & 97 & - & 27 & 31 & 0 & 28 & 31 & 0 & 28 & $2 B$ & 8 \\
\hline TROJAN & 319 & 32 & 49 & 55 & 42 & 46 & 46 & 42 & 40 & 40 & 42 & 147 & 15 & 22 & 25 & 19 & 18 & 21 & 19 & 18 & 18 & 19 \\
\hline TURKEY PT 3 & 424 & $\theta$ & 43 & 41 & 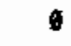 & 44 & 46 & $\theta$ & 40 & 40 & - & 192 & ต & 26 & 19 & 6 & 20 & 21 & 8 & 18 & 18 & 0 \\
\hline TURKEY PT 4 & 378 & 63 & 39 & 6 & 44 & 41 & $\theta$ & 42 & 46 & ש & 43 & 171 & 28 & 18 & 6 & 20 & 19 & $\theta$ & 19 & 18 & $g$ & 28 \\
\hline VOGTLE 1 & 6 & $\theta$ & 0 & 0 & $\$ 11$ & 65 & g & 74 & B4 & 0 & 78 & $\sigma$ & $\theta$ & - & 1 & 51 & 30 & $\theta$ & 34 & 30 & 0 & 38 \\
\hline VOCTLE 2 & 0 & 0 & - & 0 & - & 84 & 0 & 75 & 64 & 0 & 77 & 6 & $\theta$ & 0 & $\theta$ & 8 & 30 & 6 & 35 & 30 & 0 & 36 \\
\hline VT YANKEE 1 & 322 & G & 98 & 110 & t & 168 & 122 & 9 & 108 & 107 & 0 & 246 & 6 & 16 & 20 & 0 & 26 & 22 & 0 & 19 & 20 & $\theta$ \\
\hline WASH NUCLEAR 2 & $\theta$ & 122 & 151 & 145 & 126 & 135 & 153 & 145 & 134 & 134 & 142 & 6 & 22 & 28 & 27 & 23 & 24 & 27 & 26 & 24 & 24 & 25 \\
\hline WATEFFORD 3 & 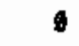 & 68 & 10 & 81 & 6 & 85 & 97 & $\theta$ & 84 & 6 & 81 & $\theta$ & 29 & 0 & 33 & $\theta$ & 35 & 40 & $\emptyset$ & 35 & $\theta$ & 34 \\
\hline WATTS RAR 1 & 6 & 6 & 6 & 6 & $g$ & 0 & 46 & 89 & 6 & 55 & 0 & $\theta$ & $\theta$ & 0 & 6 & 6 & $\theta$ & 21 & 32 & 6 & 25 & $\theta$ \\
\hline WTTSS BAR 2 & 6 & 6 & $\theta$ & 6 & $\theta$ & 0 & 48 & $\theta$ & 68 & 6 & 66 & B & $\theta$ & 6 & 6 & 0 & $\theta$ & 22 & 0 & 26 & 0 & 36 \\
\hline NMP-1 & 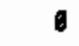 & 6 & 6 & 6 & 0 & 1 & 0 & $\theta$ & 8 & 0 & $\theta$ & 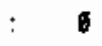 & $\theta$ & - & 0 & 0 & 6 & 0 & $B$ & $\emptyset$ & $\theta$ & $\theta$ \\
\hline$N N P-3$ & $\theta$ & 0 & 6 & $\theta$ & $\theta$ & $\theta$ & 8 & 6 & 6 & 0 & $\theta$ & $\theta$ & a & $\theta$ & 0 & 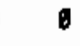 & 6 & $\theta$ & 1 & 8 & 6 & $\theta$ \\
\hline WOLF CREEX 1 & 6 & 47 & 39 & 41 & 41 & 41 & 46 & 42 & 46 & 40 & 42 & 6 & 22 & 18 & 19 & 19 & 19 & 21 & 19 & 19 & 19 & 19 \\
\hline YANKEE-ROIE 1 & 341 & 0 & 36 & 34 & 0 & 31 & 39 & B & 38 & - & 35 & 83 & 0 & 8 & 8 & $\theta$ & 7 & 9 & 0 & 7 & 8 & 8 \\
\hline ZION 1 & 510 & 58 & 57 & 6 & 61 & 81 & 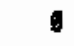 & 63 & Bo & 0 & 63 & 233 & 28 & 26 & 0 & $2 B$ & 28 & 0 & 29 & 27 & 0 & 29 \\
\hline ZION 2 & 563 & $\theta$ & 81 & 82 & $\theta$ & 84 & 89 & 1 & 60 & 60 & a & 229 & $\theta$ & $2 B$ & 28 & - & 29 & 32 & 6 & 27 & 27 & $\theta$ \\
\hline BRUNSTICX 1 PUR & 180 & $\theta$ & 0 & 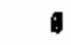 & 8 & • & 0 & 0 & 8 & $\theta$ & - & 71 & 1 & 1 & $B$ & 6 & - & 6 & 6 & 6 & 6 & $\theta$ \\
\hline BRUASTICK 2 PUR & 144 & - & - & 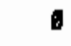 & - & 6 & 6 & ! & $\theta$ & $\theta$ & - & 66 & 0 & - & $\theta$ & 6 & - & 0 & 6 & 8 & 6 & $\theta$ \\
\hline HARRIS 1 GIR POCL & - & 6 & - & - & 日 & 6 & 6 & 6 & $\theta$ & 0 & $\sigma$ & 분 & 0 & - & 6 & 8 & 10 & 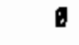 & 6 & $\theta$ & $\theta$ & $\theta$ \\
\hline MORRIS-BWR & 1527 & $\theta$ & 0 & - & 0 & - & 0 & 6 & 6 & 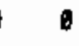 & o & 290 & B & 0 & g & 6 & 6 & 6 & 6 & $\theta$ & $\sigma$ & $\theta$ \\
\hline MORRIS-PWR & 350 & 6 & 0 & 0 & 0 & 6 & 1 & $\delta$ & 0 & 6 & a & 132 & $\theta$ & 6 & 6 & 6 & 6 & 6 & 6 & $\theta$ & $B$ & $B$ \\
\hline MEST VALLEY-B & 85 & 0 & 0 & $\theta$ & $\theta$ & 6 & 8 & 6 & 0 & 6 & 6 & 11 & 0 & 6 & 8 & 6 & 0 & 6 & 6 & 0 & $\theta$ & $\theta$ \\
\hline VEST УАЕY-P & 120 & 6 & 6 & $\theta$ & 0 & 6 & $\theta$ & 6 & 0 & b & 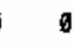 & 47 & $\theta$ & $\theta$ & 8 & 6 & 8 & 8 & $\theta$ & $\theta$ & 0 & $b$ \\
\hline GWR GENERIC & $\theta$ & $\theta$ & 0 & $\theta$ & $\theta$ & 6 & $\theta$ & 8 & $\theta$ & 6 & $\theta$ & 0 & 0 & 0 & 1 & 0 & $\emptyset$ & 6 & $\emptyset$ & $\emptyset$ & $\theta$ & 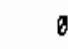 \\
\hline PWR GENERIC & - & 0 & 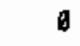 & $\theta$ & 6 & 6 & 6 & 0 & 0 & 6 & 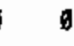 & $\theta$ & D & 6 & 6 & 0 & g & 0 & $\emptyset$ & 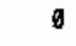 & 0 & $\emptyset$ \\
\hline TOTAL GENERIC & 0 & 0 & 0 & 0 & $\theta$ & $\theta$ & 0 & 6 & 0 & 0 & g & 1 & 0 & 6 & $\Delta$ & 0 & $\theta$ & $\theta$ & 8 & 0 & 0 & . \\
\hline
\end{tabular}


TABLE A.3. Upper Reference Case, 1985 Inventory and Projected Annual Reactor Discharges (contd)

\begin{tabular}{|c|c|c|c|c|c|c|c|c|c|c|c|c|c|c|c|c|c|c|c|c|}
\hline REACTOR & 1998 & 1997 & 1998 & $\underline{1998}$ & 2008 & 2901 & $\underline{2002}$ & 2863 & 2004 & 2985 & $\underline{1996}$ & $\underline{1997}$ & $\underline{1998}$ & 1999 & 2000 & 2001 & 2602 & 2063 & $\underline{2864}$ & 2005 \\
\hline K NUCLEAR 1 & 42 & 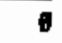 & 45 & 0 & 48 & 8 & 39 & 0 & 48 & 40 & 19 & 0 & 21 & 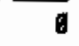 & 22 & 6 & 18 & 5 & 21 & 19 \\
\hline RK WCLEAR 2 & 50 & $\theta$ & 58 & 6 & 58 & 55 & 1 & 62 & $\theta$ & 47 & 21 & 0 & 23 & $\theta$ & 24 & 23 & $\theta$ & 28 & 6 & 29 \\
\hline V슽 1 & 54 & 59 & 6 & 58 & 63 & - & 51 & 87 & $\sigma$ & 51 & 25 & 27 & 6 & 27 & 29 & $\theta$ & 24 & 31 & 0 & 2 \\
\hline VALEY 2 & 46 & 73 & $\theta$ & 45 & 62 & 6 & 44 & $\theta$ & 68 & 41 & 21 & 34 & - & 21 & 29 & 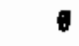 & 20 & 日 & 27 & 15 \\
\hline 3日—EONTE 1 & 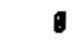 & 0 & 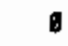 & $\theta$ & $\theta$ & a & $\$ 1$ & $\theta$ & 67 & 68 & 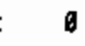 & $\theta$ & 6 & 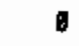 & 6 & 1 & 29 & 0 & 31 & 38 \\
\hline 昍上EDNTE 2 & 6 & $\theta$ & 6 & 39 & B & 74 & 56 & 0 & 79 & 52 & $\theta$ & 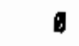 & 0 & 17 & $\theta$ & 34 & 28 & 6 & 36 & 2 \\
\hline BIG ROCK 1 & 16 & 10 & 16 & 15 & 18 & 18 & 0 & 18 & 16 & 14 & 2 & 2 & 2 & 2 & 2 & 2 & 0 & 2 & 2 & \\
\hline QRAIDWOOD 1 & - & 83 & 88 & $\theta$ & 73 & 72 & 6 & 53 & 87 & - & 0 & 35 & 29 & 0 & 31 & 30 & 0 & 22 & 28 & \\
\hline AIOHOOD 2 & 6 & 87 & 68 & 6 & 78 & 73 & 0 & 69 & 87 & $a$ & $\theta$ & 37 & 29 & $\theta$ & 33 & 31 & 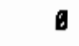 & 29 & 28 & \\
\hline GROWNS FERRY1 & 189 & 184 & 0 & 179 & 0 & 181 & 161 & g & 189 & 6 & 31 & 33 & 0 & 32 & $\theta$ & 33 & 29 & 1 & 34 & \\
\hline AROWNS FERRY2 & 170 & 183 & 6 & 179 & 0 & 182 & 162 & - & 198 & g & 31 & 33 & $\theta$ & 32 & 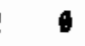 & 33 & 29 & $\theta$ & 34 & \\
\hline BROTNS FERRY3 & 176 & 0 & 185 & 178 & 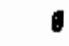 & 182 & $\theta$ & 208 & $\theta$ & 180 & 31 & $y$ & 34 & 32 & a & 33 & $\theta$ & 37 & 8 & 29 \\
\hline BRUNSWICK 1 & $\theta$ & 148 & 149 & 0 & 158 & 0 & 131 & 167 & $\theta$ & 129 & 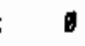 & 28 & 28 & 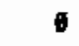 & 30 & 0 & 24 & 31 & b & 24 \\
\hline BRUNSTICK 2 & 137 & 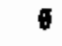 & 150 & 145 & 6 & 147 & 131 & $\theta$ & 154 & 129 & 26 & $\theta$ & 28 & 27 & 0 & 27 & 24 & 9 & 29 & 24 \\
\hline YRON 1 & 62 & 67 & - & 86 & 72 & 1 & 60 & 76 & (1) & 59 & 28 & 28 & 6 & 28 & 36 & 0 & 25 & 32 & $\theta$ & 25 \\
\hline BYROK 2 & 55 & - & 88 & 51 & $\theta$ & 72 & 49 & 6 & 67 & 48 & 23 & 0 & 29 & 22 & 0 & 36 & 21 & $a$ & 28 & 15 \\
\hline CNLAMAY 1 & 65 & 6 & 78 & 69 & 0 & 78 & 62 & 6 & 79 & 62 & 27 & 6 & 33 & 29 & $\theta$ & 32 & 26 & 6 & 33 & 26 \\
\hline XLVERT CLF 1 & 68 & & 74 & 0 & .78 & 0 & 65 & 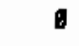 & 70 & D & 26 & 0 & 28 & 0 & 29 & $\theta$ & 24 & 9 & 29 & \\
\hline FLVERT CLF 2 & 6 & 73 & 6 & 72 & - & 72 & 6 & B4 & 0 & 84 & 6 & 27 & 6 & 27 & 6 & 27 & 0 & 32 & 0 & 2 \\
\hline SATATEA 1 & 47 & 51 & 62 & 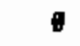 & 55 & 51 & 45 & 58 & . & 45 & 20 & 22 & 22 & $\theta$ & 23 & 22 & 19 & 25 & $\theta$ & 19 \\
\hline EATAMBA 2 & 41 & 88 & 51 & 1 & 59 & 58 & 39 & 49 & 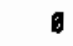 & 35 & 17 & $2 B$ & 22 & a & 25 & 24 & 16 & 21 & 0 & 15 \\
\hline CLINTON 1 & 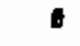 & 211 & 162 & 6 & 187 & 180 & 6 & 172 & 141 & $\theta$ & $\vartheta$ & 39 & 30 & 6 & 34 & 29 & 6 & 31 & 28 & \\
\hline COWAMCHE PK 1 & 44 & 64 & 69 & 43 & 55 & 48 & 48 & 51 & 43 & 45 & 18 & 28 & 20 & 17 & 22 & 18 & 19 & 21 & 17 & 18 \\
\hline COLANCHE PK 2 & 43 & 61 & 51 & 43 & 54 & 51 & 41 & 51 & 52 & 37 & 17 & 25 & 21 & 17 & 22 & 21 & 17 & 21 & 21 & 15 \\
\hline :OOK 1 & 59 & D & 84 & 6 & 68 & 64 & : & 73 & 0 & 56 & 27 & 0 & 29 & 6 & 31 & 29 & 0 & 34 & 0 & 26 \\
\hline ook 2 & $\theta$ & 76 & 0 & 69 & 75 & 0 & 62 & 6 & 73 & 62 & 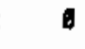 & 28 & g & 28 & 36 & $\theta$ & 25 & 0 & 29 & 25 \\
\hline COOPER STN & 86 & 90 & 94 & B7 & 96 & 68 & 79 & 181 & 92 & 76 & 18 & 16 & 17 & 16 & 17 & 16 & 14 & $1 B$ & 17 & 14 \\
\hline CRYSTAL RYR 3 & $\theta$ & 58 & 0 & 57 & 9 & 57 & $\theta$ & 85 & 0 & 51 & 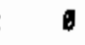 & 27 & $\theta$ & 26 & $\theta$ & 26 & 6 & 30 & 9 & 24 \\
\hline AVIS-8ESSE I & 45 & 49 & 6 & 47 & 52 & 0 & 42 & 55 & 6 & 42 & 21 & 23 & 6 & 22 & 24 & 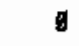 & 20 & 26 & 6 & 20 \\
\hline DIAELO CANYON-1 & 6 & 83 & 0 & 85 & 曰 & 82 & $\theta$ & 98 & 0 & 72 & 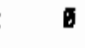 & 38 & 0 & 39 & 0 & 38 & $\theta$ & 45 & 0 & 33 \\
\hline DIABR CANYON-2 & 68 & $\theta$ & 84 & - & 92 & G & 73 & 0 & 96 & 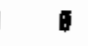 & 37 & 0 & 39 & 6 & 42 & 6 & 34 & 0 & 41 & \\
\hline ORESDEN 1 & 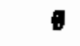 & - & D & 0 & - & $\theta$ & D & $a$ & 6 & 0 & 6 & g & 0 & 6 & - & 0 & $\theta$ & 6 & 9 & \\
\hline RESDEN 2 & $\theta$ & 128 & 128 & o & 136 & 125 & 0 & 142 & 132 & 0 & 9 & 21 & 21 & D & 23 & 21 & 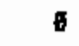 & 24 & 22 & \\
\hline DRESDEN 3 & 117 & 1 & 128 & 124 & 0 & 125 & 112 & - & 131 & 169 & 28 & 6 & 21 & 21 & 1 & 21 & 19 & 6 & 22 & 18 \\
\hline DUANE ARNOLD & 94 & 98 & a & 95 & 189 & 10 & 86 & 168 & 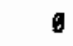 & 89 & 17 & 17 & 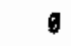 & 17 & 19 & 6 & 15 & 18 & 6 & 16 \\
\hline ENRICO FERMI-2 & 214 & 0 & 238 & 6 & 247 & 9 & 290 & 259 & 6 & 204 & 39 & 6 & 43 & 0 & 45 & 6 & 37 & 47 & 9 & 37 \\
\hline ARLEY 1 & $\sigma$ & 58 & 58 & 6 & 62 & 54 & $\theta$ & 85 & 58 & $\theta$ & 9 & 27 & 26 & \% & 29 & 25 & 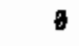 & 30 & 27 & \\
\hline FARLEY 2 & 51 & 日 & $5 B$ & 54 & - & 58 & 48 & $a$ & 60 & 48 & 24 & $\theta$ & 27 & 25 & $\theta$ & 27 & 22 & 0 & 28 & 22 \\
\hline FITZPATRICK & 134 & 140 & 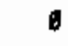 & 137 & 158 & 6 & 124 & 159 & 6 & 123 & 24 & 25 & $\theta$ & 24 & 27 & $B$ & 22 & 28 & $\theta$ & 22 \\
\hline FORT CALHOUN & 32 & 38 & 6 & 33 & 38 & 0 & 31 & 41 & 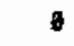 & 30 & 12 & 13 & $a$ & 12 & 14 & 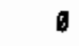 & 11 & 15 & 6 & 11 \\
\hline GIKNA & 23 & 25 & 28 & 25 & 27 & 25 & 23 & 29 & 27 & 22 & $B$ & 8 & 9 & B & 9 & $B$ & B & 16 & 9 & \\
\hline GRAND GUF 1 & 197 & D & 215 & 289 & $\theta$ & 211 & 188 & a & 226 & 184 & 35 & 6 & 38 & 37 & 6 & 37 & 33 & 9 & 39 & 32 \\
\hline GRAND GLLF 2 & $\theta$ & - & B & g & 6 & 8 & 209 & 386 & 6 & 128 & ஏ & 9 & 0 & 8 & 0 & b & 38 & 58 & 9 & 23 \\
\hline HADOAK NECK & 0 & 42 & 40 & 42 & $\theta$ & 42 & 38 & 48 & 9 & 37 & 0 & 17 & 16 & 17 & $\theta$ & 17 & 16 & 29 & 8 & 15 \\
\hline |ARRIS 1 & 0 & 53 & 8 & 33 & 45 & 9 & 32 & 39 & $\theta$ & 29 & 8 & 25 & b & 15 & 21 & b & 15 & 18 & 9 & 13 \\
\hline IATCH 1 & 138 & 148 & 0 & 145 & 158 & 8 & 130 & 186 & 0 & 129 & 26 & 27 & $\theta$ & 27 & 29 & 6 & 24 & 31 & a & 24 \\
\hline HATCH 2 & 138 & $b$ & 148 & 144 & $a$ & 146 & 136 & 6 & 153 & 128 & 25 & - & 27 & 27 & - & 27 & 24 & $b$ & $2 B$ & 24 \\
\hline HOPE CREEX & 189 & 0 & 215 & 162 & 6 & 216 & 158 & 0 & 210 & 144 & 31 & 0 & 39 & 29 & 9 & 39 & 28 & 6 & 38 & \\
\hline
\end{tabular}


TABLE A.3. Upper Reference Case, 1985 Inventory and Projected Annual Reactor Discharges (contd)

\begin{tabular}{|c|c|c|c|c|c|c|c|c|c|c|c|c|c|c|c|c|c|c|c|c|c|}
\hline \multirow[b]{2}{*}{ REACTOR } & \multicolumn{10}{|c|}{ ASSBIRIES } & & \multicolumn{10}{|c|}{ UTIHU } \\
\hline & $\underline{1998}$ & $\underline{1997}$ & $\underline{1998}$ & $\underline{1999}$ & 2869 & 2001 & $\underline{2002}$ & 2903 & $\underline{2064}$ & 2895 & & 1996 & 1997 & 1998 & 1999 & 2000 & 2901 & $\underline{2602}$ & 20963 & $\underline{2604}$ & $\underline{2065}$ \\
\hline HYBOLDT BAY & 7 & 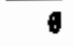 & 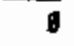 & 9 & 6 & 0 & 9 & 0 & 0 & 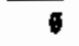 & & 0 & 1 & 0 & 6 & 6 & 6 & 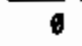 & 0 & 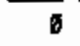 & 0 \\
\hline INDIAN PT 1 & 6 & - & - & - & 6 & ] & 6 & 0 & 1 & 6 & & - & 6 & 0 & 6 & 6 & 6 & 0 & B & 6 & $\theta$ \\
\hline INDIN PT 2 & 6 & 54 & 6 & 54 & 58 & ] & 48 & 6 & 57 & 48 & & $\theta$ & 24 & 6 & 24 & 28 & 6 & 22 & $\theta$ & 28 & 22 \\
\hline INDIM PT 3 & 5 & 81 & 81 & 6 & 85 & - & 54 & 89 & - & 53 & & B & 28 & 28 & 0 & 38 & 6 & 26 & 31 & 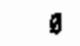 & 24 \\
\hline KEUALNEE & 27 & 30 & 36 & 29 & 32 & 29 & 26 & 34 & 31 & 28 & & 10 & 11 & 11 & 11 & 12 & 11 & 10 & 13 & 12 & 16 \\
\hline LACROSSE & 18 & 6 & 10 & $\theta$ & 21 & 6 & 16 & 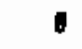 & 20 & - & & 3 & $\sigma$ & 3 & 1 & 3 & 0 & 3 & 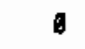 & 3 & 6 \\
\hline LASA $\amalg E$ CTY 1 & 183 & 6 & 177 & 173 & 0 & 175 & 158 & $\theta$ & 183 & 153 & & 38 & 6 & 32 & 31 & 0 & 32 & 28 & $\theta$ & 33 & $2 B$ \\
\hline LSA $\perp E ~ C T Y 2$ & 1 & 176 & 177 & 1 & 188 & 175 & 0 & 199 & 183 & 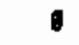 & : & $\forall$ & 32 & 32 & 0 & 34 & 32 & 0 & 36 & 93 & 0 \\
\hline LIUERICK 1 & 101 & $\theta$ & 175 & 170 & 0 & 171 & 153 & 6 & 180 & 150 & & 29 & 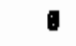 & 31 & 30 & $\theta$ & 36 & 27 & - & 32 & 27 \\
\hline LIMERICK 2 & $\theta$ & 284 & 0 & 174 & 233 & 0 & IAI & 6 & 238 & 110 & & 6 & 52 & $\theta$ & 31 & 41 & 0 & 32 & $\theta$ & 42 & 20 \\
\hline WAINE YANKEE & 54 & 59 & 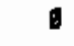 & 57 & 63 & $\theta$ & 52 & 67 & 0 & 51 & & 21 & 22 & $\theta$ & 22 & 24 & 8 & 20 & 25 & 0 & 19 \\
\hline NCQUIRE 1 & 47 & 51 & B & 50 & 55 & 51 & ( & 58 & 53 & 45 & : & 29 & 22 & 0 & 21 & 23 & 22 & $\theta$ & 25 & 22 & 19 \\
\hline KCOUIRE 2 & 47 & 51 & 52 & 50 & $\theta$ & 58 & 45 & 58 & $\theta$ & 45 & : & 20 & 22 & 22 & 21 & 8 & 21 & 19 & 25 & 8 & 19 \\
\hline NIDLAND-1 & $\theta$ & $\theta$ & 0 & 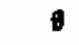 & 6 & $\theta$ & B & 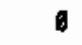 & 0 & 25 & : & 0 & $\theta$ & 0 & $B$ & b & $b$ & 6 & 6 & 8 & a \\
\hline NIDLAND-2 & $\theta$ & $\theta$ & 68 & - & 59 & 58 & 6 & 54 & 57 & 6 & : & 6 & 9 & 28 & 6 & 27 & 27 & 0 & 25 & 26 & 0 \\
\hline NILSTONE 1 & 149 & - & 183 & - & 171 & 160 & 6 & 189 & $\theta$ & 146 & : & 26 & $\theta$ & 29 & $\theta$ & 30 & 28 & 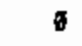 & 32 & $\pi$ & 25 \\
\hline MIUSTONE 2 & 57 & 59 & 58 & 9 & 82 & 58 & 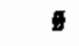 & 88 & 69 & 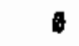 & : & 23 & 24 & 24 & 0 & 25 & 24 & 0 & 27 & 24 & $\theta$ \\
\hline NIЦSTONE 3 & 58 & 0 & 68 & 57 & 6 & 76 & B4 & - & 87 & 58 & $:$ & 27 & 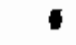 & 31 & 28 & 曰 & 32 & 38 & 0 & 31 & 27 \\
\hline MONTICELO & $B 6$ & 0 & 67 & 83 & 92 & $\bullet$ & 77 & 98 & 96 & 6 & : & 14 & $\theta$ & 15 & 14 & 18 & 0 & 13 & 17 & 15 & $\theta$ \\
\hline NINE NILE PT1 & 141 & 6 & 145 & 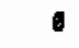 & 159 & 6 & 131 & $\theta$ & 153 & $\theta$ & : & 24 & 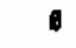 & 25 & : & 27 & - & 22 & $B$ & 28 & 0 \\
\hline NINE NILE PT2 & 180 & 0 & 218 & - & 222 & 6 & 165 & - & 221 & - & : & 32 & 1 & $\mathbf{2 3}$ & - & 38 & 6 & $2 B$ & 1 & 38 & 0 \\
\hline NORTH ANKA 1 & 48 & 52 & $\theta$ & 50 & 55 & 0 & 45 & 58 & 6 & 45 & $:$ & 22 & 24 & - & 23 & 25 & 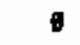 & 21 & 27 & 6 & 21 \\
\hline NORTH ANNA 2 & 49 & $\theta$ & 52 & 50 & $\boldsymbol{\theta}$ & 51 & 45 & g & 53 & 45 & : & 23 & g & 24 & 23 & 6 & 24 & 21 & $\bullet$ & 24 & 21 \\
\hline OCONEE 1 & 45 & 48 & 6 & 47 & 52 & $4 B$ & • & 54 & 60 & 42 & : & 21 & 22 & 6 & 22 & 24 & 22 & 0 & 25 & 23 & 19 \\
\hline OCONEE 2 & 45 & 48 & 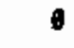 & 47 & 51 & 48 & $\theta$ & 55 & 42 & 42 & : & 21 & 22 & - & 22 & 24 & 22 & 0 & 25 & 19 & 19 \\
\hline OCONEE 3 & $\theta$ & 48 & 49 & 47 & $\theta$ & 47 & 42 & $\theta$ & 50 & 42 & $:$ & $\theta$ & 22 & 23 & 22 & - & 22 & 19 & 6 & 23 & 19 \\
\hline OYSTER CRK 1 & 130 & 120 & 6 & 128 & - & 139 & 121 & 6 & 147 & 118 & : & 23 & 21 & $\theta$ & 23 & v & 25 & 21 & 6 & 28 & 21 \\
\hline PALISADES & 50 & 0 & 55 & 53 & $\theta$ & 54 & $\theta$ & 61 & 58 & o & : & 18 & 0 & 21 & 21 & E & 21 & 6 & 24 & 22 & 6 \\
\hline PALO VERDE I & 47 & 51 & 52 & 50 & 55 & 51 & 45 & 58 & 63 & 45 & : & 21 & 22 & 23 & 22 & 24 & 22 & 20 & 25 & 23 & 26 \\
\hline PALO VERDE 2 & 44 & 68 & 58 & 43 & 57 & 51 & 48 & 48 & 43 & 44 & $:$ & 19 & 29 & 22 & 19 & 25 & 22 & 21 & 21 & 19 & 19 \\
\hline PALO VERDE 3 & 40 & 87 & 52 & 39 & 59 & 55 & 39 & 52 & 52 & 35 & : & 17 & 29 & 23 & 17 & 28 & 24 & 17 & 23 & 23 & 15 \\
\hline PEACHBOTTOU 2 & 169 & 183 & - & 179 & 195 & 6 & 161 & 205 & $\theta$ & 158 & : & 30 & 32 & $\theta$ & 32 & 35 & 6 & 29 & 38 & 0 & 28 \\
\hline PEACHBOTTON 3 & - & 172 & 177 & $\bullet$ & 182 & $\$ 72$ & 6 & 194 & 179 & - & : & $\theta$ & 36 & 31 & 0 & 32 & 30 & 0 & 34 & 32 & 0 \\
\hline PERRY I & $\theta$ & 231 & 196 & - & 289 & 169 & 6 & 203 & 183 & - & : & 6 & 42 & 36 & - & 38 & 31 & 0 & 37 & 39 & $\theta$ \\
\hline PERRY 2 & 223 & 244 & - & 185 & 280 & 6 & 260 & 333 & - & 127 & : & 41 & 45 & - & 36 & 47 & 6 & 38 & 61 & 6 & 23 \\
\hline PILGRIU 1 & 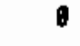 & 154 & 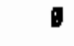 & 152 & $\theta$ & 156 & 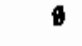 & 173 & 6 & 136 & & - & 27 & ( & 27 & 0 & 28 & 9 & 36 & 0 & 24 \\
\hline POINT GEACH 1 & 23 & 25 & 26 & 26 & 28 & 25 & 23 & 29 & 26 & 23 & & 8 & 9 & 9 & 9 & 10 & 9 & 8 & 10 & 9 & 8 \\
\hline POINT BEACH 2 & 24 & 26 & 25 & 25 & 27 & 25 & 22 & 28 & 27 & 22 & & 8 & 9 & 9 & 9 & 10 & $\theta$ & 8 & 10 & 10 & 8 \\
\hline PRAIRIE ISL 1 & 29 & 32 & 32 & 32 & 34 & 0 & 28 & 36 & 33 & 28 & $:$ & 10 & 11 & 11 & 11 & 12 & 0 & 18 & 13 & 12 & 10 \\
\hline PRAIRIE ISL 2 & $\theta$ & 32 & 32 & 31 & 34 & 32 & 28 & 37 & 33 & 28 & & D & 11 & 11 & 11 & 12 & 11 & 10 & 13 & 12 & 10 \\
\hline QUAD CITIES 1 & 118 & 6 & 129 & 128 & 0 & 127 & 113 & g & 134 & 112 & & 21 & 0 & 23 & 22 & 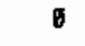 & 22 & 20 & 0 & 24 & 26 \\
\hline QUAD CITIES 2 & 6 & 128 & 128 & 0 & 138 & 128 & 0 & 145 & 133 & a & & $\theta$ & 23 & 23 & $\theta$ & 24 & 23 & 0 & 26 & 23 & $\theta$ \\
\hline RANCHO SECD-1 & 0 & 46 & 49 & a & 52 & 45 & $\emptyset$ & 55 & 50 & 8 & & $a$ & 21 & 23 & 6 & 24 & 21 & 6 & 25 & 23 & 0 \\
\hline ROBIHSDN 2 & 36 & 48 & 6 & 37 & 41 & 6 & 43 & 44 & 40 & v & & 16 & 20 & $\theta$ & 16 & 18 & 0 & 18 & 19 & 17 & 0 \\
\hline RVR BEND 1 & 155 & 167 & a & 164 & 186 & 0 & 148 & 169 & 0 & 145 & & 29 & 31 & $\theta$ & 30 & 33 & 0 & 27 & 35 & 6 & 27 \\
\hline SALEM UNIT 2 & 0 & 77 & 72 & 6 & 76 & 67 & $\emptyset$ & 77 & 76 & 0 & & 0 & 35 & 33 & 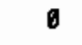 & 35 & 31 & 0 & 35 & 32 & $\theta$ \\
\hline SALEN 1 & 60 & D & 72 & 76 & 1 & 67 & 59 & 0 & 76 & 59 & & 28 & 6 & 33 & 32 & 0 & 31 & 27 & 0 & 32 & 27 \\
\hline SAN ONOFRE 1 & 39 & $\theta$ & 42 & $\theta$ & 44 & 6 & 37 & 0 & 44 & $\theta$ & & 14 & 6 & 16 & $\theta$ & 18 & 0 & 14 & 6 & 16 & 0 \\
\hline
\end{tabular}



TABLE A.3. Upper Reference Case, 1985 Inventory and Projected Annual Reactor
Discharges (contd)

ASSEUARE

VTIHN

\begin{tabular}{|c|c|c|c|c|c|c|c|c|c|c|c|c|c|c|c|c|c|c|c|c|}
\hline REACTOR & 1998 & 1897 & 1998 & 1989 & 2090 & 2091 & $\underline{2092}$ & 2003 & 2004 & $\underline{2005}$ & 1998 & $\underline{1997}$ & 1898 & 1989 & $\underline{2008}$ & 2001 & 2042 & 2803 & 2084 & $\underline{2005}$ \\
\hline SAK ONOFRE 2 & 68 & 5 & 66 & 6 & 78 & 6 & 83 & 6 & 74 & 6 & 28 & 6 & 28 & 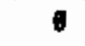 & 32 & 0 & 27 & 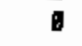 & 32 & 6 \\
\hline SAN ONBFRE 3 & 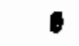 & 71 & 0 & 64 & 6 & 71 & 6 & 81 & 6 & 63 & 6 & 38 & $\theta$ & 27 & $\theta$ & 30 & g & 35 & 8 & 27 \\
\hline SEABRCOK 1 & 42 & 6 & 52 & 39 & 54 & 51 & 38 & 6 & 50 & 35 & 18 & 0 & 24 & 18 & 25 & 24 & 18 & 0 & 23 & 16 \\
\hline SEABROEX 2 & 6 & 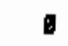 & 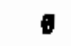 & 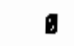 & 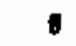 & 0 & $\theta$ & 61 & 58 & 80 & $\theta$ & : & 6 & 0 & 6 & $\theta$ & 0 & 28 & 27 & $2 B$ \\
\hline SERUOYAH 1 & 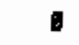 & 84 & 65 & 6 & 89 & g & 57 & $7 \mathrm{~s}$ & a & 56 & a & 29 & 30 & 6 & 31 & $B$ & 26 & 34 & 6 & 26 \\
\hline SERUOYAH 2 & 6 & 64 & $\theta$ & 63 & 69 & 6 & 58 & 6 & 87 & 56 & 6 & 30 & 8 & 29 & 32 & a & 28 & 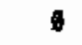 & 31 & 26 \\
\hline SHOREHAN & 122 & 176 & 0 & 115 & 136 & 日 & 136 & 115 & 6 & 128 & 22 & 31 & 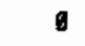 & 21 & 25 & $\theta$ & 24 & 21 & छ & 23 \\
\hline SOUTH TEXAS, UNIT 1 & 35 & 58 & 41 & 35 & 58 & 46 & 39 & 39 & 35 & 38 & 19 & 31 & 22 & 19 & 27 & 24 & 21 & 21 & 19 & 19 \\
\hline SOUTH TEXAS, UNIT 2 & 33 & 58 & 42 & 32 & 51 & 48 & 30 & 43 & 42 & 28 & 18 & 32 & 23 & 17 & 28 & 28 & 18 & 23 & 23 & 15 \\
\hline ST LUCIE 1 & 58 & 81 & a & 59 & 86 & 6 & 54 & 69 & 0 & 53 & 21 & 23 & - & 23 & 25 & 9 & 21 & 26 & $\theta$ & 29 \\
\hline ST LUXIE 2 & 53 & - & 58 & 57 & 6 & 58 & 51 & $\theta$ & 60 & 50 & 21 & - & 23 & 22 & 6 & 23 & 26 & 8 & 23 & 19 \\
\hline SIMAER 1 & 50 & 54 & 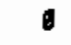 & 53 & 59 & $\theta$ & 46 & 62 & - & 47 & 23 & 25 & 6 & 25 & 27 & $\theta$ & 22 & 29 & $\theta$ & 22 \\
\hline SURRY 1 & 39 & 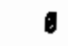 & 43 & 41 & 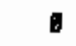 & 41 & 37 & 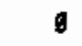 & 43 & 38 & 18 & 6 & 20 & 19 & 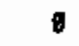 & 19 & 17 & 8 & 29 & 17 \\
\hline SURRY 2 & - & 43 & 43 & a & 44 & 42 & 6 & 48 & 44 & 5 & 6 & 20 & 29 & 6 & 29 & 19 & 6 & 22 & 20 & $\theta$ \\
\hline SUSQUEYAKNA 1 & 168 & 6 & 182 & 175 & - & 177 & 158 & 6 & 107 & 158 & 29 & 6 & $3 !$ & 36 & 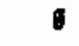 & 31 & 27 & 6 & 32 & 27 \\
\hline SUSQUEHANKA 2 & 186 & 280 & 6 & 175 & 192 & 0 & 150 & 291 & 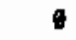 & 158 & 29 & 31 & 6 & 39 & 39 & $\theta$ & 27 & 35 & 0 & 27 \\
\hline 3 MILE ISL 1 & 53 & 6 & 58 & 50 & 6 & 58 & 51 & g & 89 & 51 & 25 & 6 & 27 & 26 & 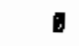 & 27 & 24 & g & 28 & 24 \\
\hline TROJAN & 36 & 39 & 39 & 38 & 41 & 38 & 34 & 44 & 46 & 34 & 16 & 16 & 18 & 18 & 19 & 18 & 18 & 20 & 18 & 16 \\
\hline TURKEY PT 3 & 35 & 38 & 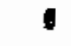 & 38 & 41 & a & 34 & 44 & $\theta$ & 33 & 16 & 17 & 0 & 17 & 19 & b & 16 & 20 & $\theta$ & 15 \\
\hline TURKEY PT 4 & 38 & 6 & 39 & 38 & - & 38 & 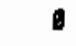 & 44 & 49 & 6 & 17 & 0 & 18 & 17 & 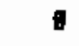 & 17 & 0 & 29 & 18 & 6 \\
\hline YOGTLE 1 & 58 & 日 & 88 & 57 & $\theta$ & 87 & 63 & 1 & 67 & 58 & 27 & : & 31 & 28 & 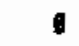 & 31 & 29 & $\theta$ & 31 & 27 \\
\hline VOGLE 2 & 59 & 6 & 20 & 58 & 4 & 67 & 85 & $\theta$ & 67 & 58 & 27 & 6 & 32 & 26 & - & 31 & 30 & b & 31 & 27 \\
\hline VT YANKEE 1 & 95 & 162 & 6 & 89 & 110 & 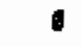 & 80 & 115 & 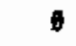 & 88 & 17 & 18 & $\theta$ & 18 & 26 & $\theta$ & 17 & 21 & 6 & 16 \\
\hline WASH NUCLEAR 2 & 128 & 128 & 130 & 125 & 138 & 128 & 113 & 145 & 134 & 111 & 21 & 23 & 23 & 22 & 24 & 23 & 20 & $2 B$ & 24 & 28 \\
\hline WATERFORD 3 & 74 & 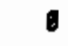 & 74 & 79 & 0 & 73 & 71 & - & 77 & 71 & 31 & 0 & 31 & 33 & 0 & 30 & 36 & $\theta$ & 32 & 30 \\
\hline WATTS BAR 1 & 58 & 83 & 0 & 50 & 6 & 72 & 54 & 8 & 55 & 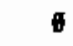 & 28 & 38 & 0 & 23 & 6 & 33 & 25 & 6 & 25 & $\theta$ \\
\hline WATTS BAR 2 & 55 & $\theta$ & 62 & 6 & 74 & 68 & - & 80 & 6 & 55 & 25 & $\theta$ & 29 & 9 & 34 & 31 & 6 & 28 & 6 & 25 \\
\hline$N N P-1$ & 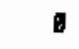 & 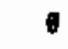 & 0 & 0 & $\theta$ & 0 & 6 & $\theta$ & 71 & 44 & 0 & 6 & 9 & 0 & 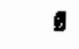 & 6 & 6 & 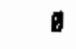 & 32 & 26 \\
\hline INP-3 & 57 & 111 & 62 & 51 & 76 & 88 & 53 & 108 & 73 & 32 & 24 & 45 & 25 & 22 & 33 & 30 & 23 & 47 & 32 & 14 \\
\hline MOLF CREEK 1 & 36 & 38 & 38 & 38 & 41 & $3 B$ & 34 & 44 & 40 & 33 & 17 & 18 & 18 & 18 & 19 & 18 & 16 & 20 & 19 & 15 \\
\hline YANKEE-ROWE 1 & 27 & a & 78 & g & 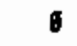 & - & a & 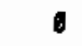 & 6 & a & 6 & 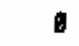 & 17 & 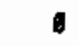 & 6 & 0 & 5 & 0 & 0 & $\theta$ \\
\hline ZION 1 & 63 & 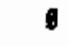 & 58 & 57 & - & 57 & 51 & $\bullet$ & 80 & 51 & 24 & $\theta$ & 27 & 28 & $\theta$ & 28 & 23 & b & 27 & 23 \\
\hline ZICN 2 & 53 & 58 & 6 & 68 & 61 & $\boldsymbol{\sigma}$ & 51 & 86 & 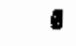 & 66 & 24 & 27 & 6 & 28 & 28 & - & 23 & 30 & $\theta$ & 23 \\
\hline BRUNSTICK I PTR & $\theta$ & 0 & 6 & $\bullet$ & 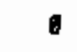 & 6 & 0 & 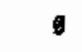 & 6 & $\theta$ & $\theta$ & 0 & 6 & 0 & - & פ & 0 & 。 & $\theta$ & 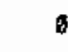 \\
\hline BRUNSTICK 2 PWR & b & 0 & - & 6 & 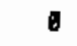 & 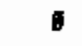 & 6 & - & 8 & 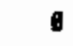 & - & 6 & 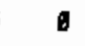 & 6 & 0 & $\theta$ & 9 & 0 & 8 & $\theta$ \\
\hline HARRIS 1 BIR PODL & 0 & 6 & 6 & - & $\sqrt{6}$ & - & 6 & $\theta$ & 0 & 6 & 6 & $\theta$ & 9 & 1 & 6 & $\theta$ & b & 9 & $\emptyset$ & $\theta$ \\
\hline MORRIS-BWR & 9 & 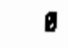 & 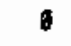 & 0 & 1 & 6 & 6 & a & 0 & 0 & $\theta$ & $g$ & b & 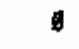 & 6 & 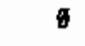 & $\theta$ & 6 & $\theta$ & 8 \\
\hline NORRIS-PWR & 6 & 9 & 0 & 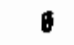 & 0 & 6 & $\theta$ & 0 & $\theta$ & 6 & 6 & 6 & 0 & 6 & 9 & 6 & 6 & 0 & $B$ & $\theta$ \\
\hline WEST VАШЕY - В & - & 6 & 9 & 0 & 6 & 8 & 9 & - & 9 & 8 & 0 & 6 & a & $\theta$ & 6 & 0 & 6 & a & 9 & 10 \\
\hline WEST VAUEY-P & $\theta$ & - & ( & 0 & $\theta$ & פ & 6 & 6 & 6 & 0 & 6 & 0 & 0 & 8 & 9 & 6 & 0 & $\theta$ & $\theta$ & $\theta$ \\
\hline BIR GENERIC & 6 & 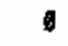 & 0 & 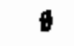 & 6 & 0 & $\theta$ & 262 & 213 & 537 & 0 & 8 & $\theta$ & 6 & $\theta$ & 4 & $\theta$ & 37 & 39 & 97 \\
\hline PWR GENERIC & 0 & 0 & 6 & 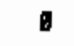 & 0 & 0 & 0 & 76 & 322 & 351 & 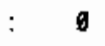 & v & $a$ & 9 & $\theta$ & 8 & 0 & 35 & 148 & 161 \\
\hline TOTAL GENERIC & $\theta$ & a & 8 & 6 & 日 & 9 & D & 278 & 535 & 888 & $B$ & 6 & 0 & $B$ & $g$ & 0 & 8 & 72 & 187 & 258 \\
\hline
\end{tabular}


IABLE A.3. Upper Reference Case, 1985 Inventory and Projected Annual Reactor Discharges (contd)

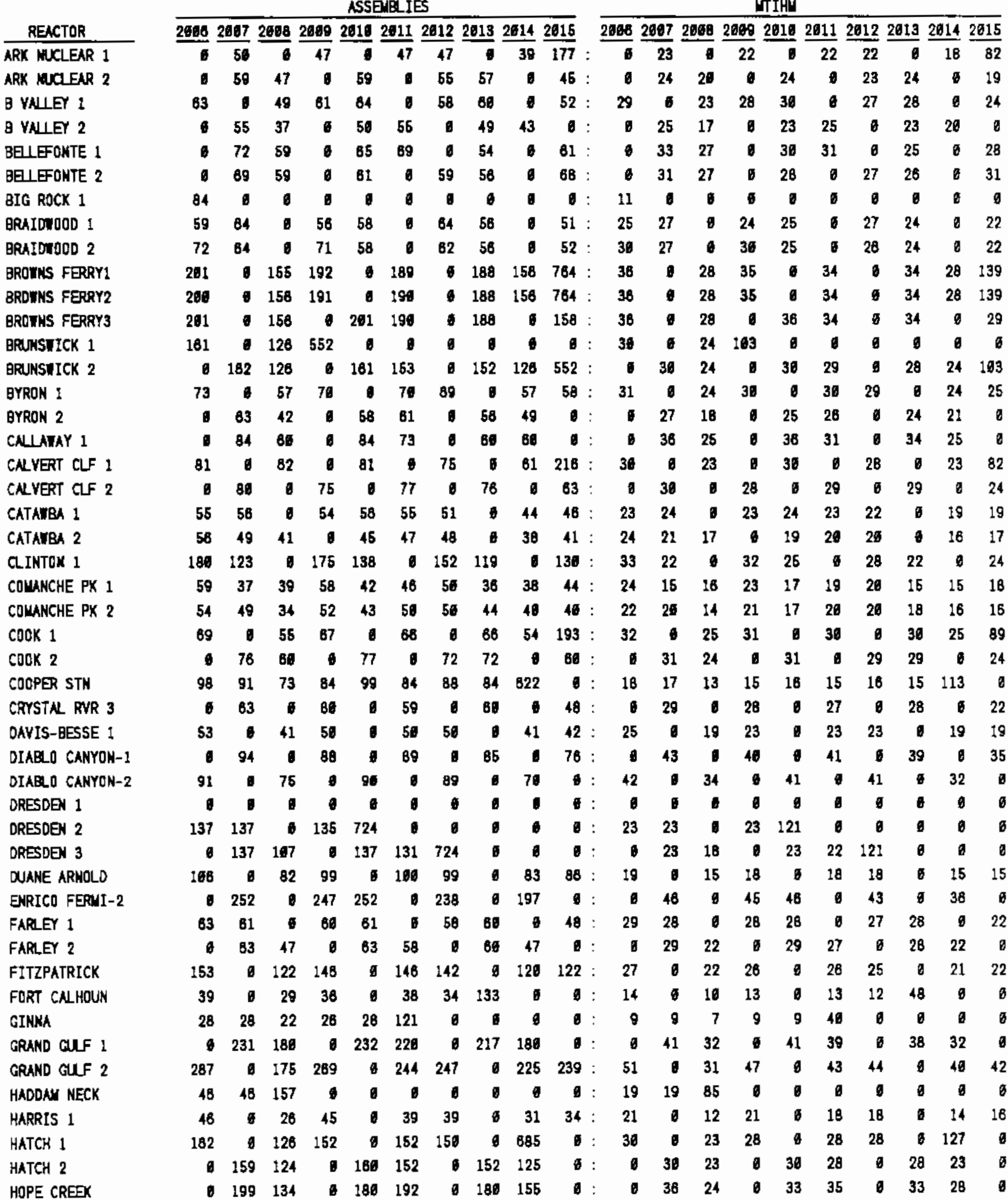


IABLE A.3. Upper Reference Case, 1985 Inventory and Projected Annual Reactor Discharges (contd)

ASSEMALIES

REACTOR

HULBQLDT BAY INDIAN PT 1 INDIAN PT 2 INDIAN PT 3 KEVALNEE

LACROSSE

LASALIE CTY 1

LASALLE CTY 2

LIUERICK 1

LIUERICK 2

WAINE YANKEE

WCAJIRE 1

WCOIRE 2

UIQAND-1

IIDAND-2

UIUSTONE 1

MILSTONE 2

MIUSTONE 3

MONTICELC

NINE WILE PT1

NINE NILE PT?

NORTH ANNA 1

NORTH ANNA 2

OCGNEE 1

OCONEE 2

OCONEE 3

OYSTER CRK 1

PALISADES

PND VERDE 1

PHLO VERDE 2

PALO VERDE 3

PEACHOCTTOH 2

PEXCHEOTTON 3

PERRY 1

PERRY 2

PILGRIN 1

POINT BEACH 1

POINT REACH 2

PRAIRIE ISL 1

PRAIRIE ISL 2

QUAO CITIES 1

QUAD CITIES 2

RANCHO SECO-1

ROAINSON 2

RVR BENO 1

SALEN UNIT 2

SALEN 1

SAK ONOFRE 1

\begin{tabular}{|c|c|c|c|c|c|c|c|c|c|c|c|c|c|c|c|c|c|c|c|}
\hline \multicolumn{10}{|c|}{ ASSEMALIES } & \multicolumn{10}{|c|}{ vTIHN } \\
\hline 196 & 2007 & $\underline{2068}$ & 2069 & 2010 & 2611 & $\underline{2612}$ & $\underline{2613}$ & $\underline{2014}$ & 2015 & 2600 & 2607 & $\underline{2608}$ & 20E9 & 2010 & $\underline{2011}$ & 2012 & 2013 & $\underline{2014}$ & 2025 \\
\hline 7 & 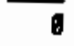 & 0 & 0 & 7 & 0 & 1 & 8 & 0 & 8 & 0 & 6 & 1 & 7 & 0 & 0 & 6 & 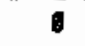 & 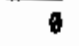 & \\
\hline E & 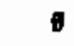 & 6 & B & 6 & 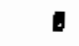 & 6 & D & b & ต : & 6 & 0 & 6 & $\theta$ & $\theta$ & 0 & 1 & 6 & 0 & \\
\hline 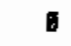 & 60 & - & 55 & 80 & t & 54 & 9 & 238 & $6:$ & 0 & 27 & a & 25 & 27 & - & 24 & 0 & 197 & \\
\hline 0 & 67 & 52 & 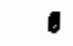 & 97 & - & 01 & 82 & 6 & 52 & 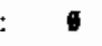 & 31 & 24 & 6 & 31 & 0 & 28 & 28 & a & 24 \\
\hline 33 & 33 & 25 & 30 & 32 & 31 & 31 & 29 & 25 & 121 & 13 & 13 & & 11 & 12 & 12 & 12 & 11 & $g$ & 46 \\
\hline 21 & 10 & 18 & 72 & 0 & 6 & 0 & 6 & 0 & 0: & 3 & 0 & 3 & 8 & $\theta$ & $\theta$ & 0 & $\theta$ & b & \\
\hline 0 & 191 & 147 & 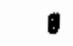 & 198 & 182 & 6 & 183 & 149 & 0 & 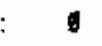 & 35 & 27 & $g$ & 35 & 33 & 9 & 33 & 27 & \\
\hline 190 & 191 & ต & 185 & 192 & • & 178 & 181 & 1 & 153 & 35 & 35 & 8 & 34 & 35 & $\boldsymbol{v}$ & 32 & 33 & & 28 \\
\hline a & 187 & 146 & D & 188 & 179 & 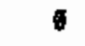 & 177 & 147 & 0 & 6 & 33 & 28 & $\theta$ & 33 & 32 & - & 31 & 26 & \\
\hline 0 & 255 & 0 & 227 & 158 & 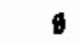 & 218 & 6 & 183 & 204 & 8 & 46 & & 40 & 28 & $\pi$ & 38 & 0 & 32 & 36 \\
\hline 83 & 1 & 50 & 59 & 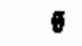 & 80 & 217 & 0 & 6 & $\boldsymbol{\theta}:$ & 24 & 0 & 19 & 22 & 6 & 23 & 2 & B & $\theta$ & \\
\hline 58 & 0 & 43 & 54 & 55 & $\theta$ & 51 & 53 & 44 & 44 & 24 & 8 & 18 & 23 & 23 & 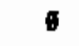 & 2 & 22 & 19 & 19 \\
\hline 57 & 58 & 43 & 0 & 58 & 54 & 51 & $\theta$ & 44 & 44: & 24 & 24 & 8 & 0 & 24 & 23 & 22 & 0 & 19 & 19 \\
\hline 31 & 28 & 24 & 31 & 25 & 28 & 29 & 28 & 27 & $29:$ & : & 9 & & 10 & 8 & & & 9 & & \\
\hline 89 & 52 & 44 & $\theta$ & 47 & 53 & 0 & 50 & 52 & 54: & 28 & 24 & 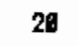 & $\theta$ & 22 & 5 & . & 23 & 4 & 25 \\
\hline$\theta$ & 175 & 136 & 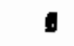 & i78 & $58 \%$ & 0 & 0 & $\theta$ & : : & : & 31 & 4 & 0 & 31 & 183 & g & 6 & 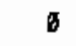 & \\
\hline 64 & 63 & 0 & 59 & 84 & 0 & 58 & 59 & 0 & $50:$ & 28. & 28 & 6 & 24 & 28 & 6 & 24 & 24 & $\emptyset$ & 20 \\
\hline 6 & 64 & 52 & 0 & 58 & 59 & 0 & 58 & 50 & 0 & 6 & 30 & 24 & $a$ & 27 & 27 & 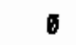 & 28 & 23 & \\
\hline 95 & 9 & & 91 & 0 & 91 & 484 & 6 & 0 & : & 18 & & & 18 & & & & & & \\
\hline 161 & & 127 & 0 & 159 & 532 & 6 & 0 & 0 & 0: & 28 & $A$ & & 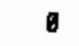 & 27 & 98 & & $\emptyset$ & $a$ & \\
\hline 238 & 0 & 140 & 6 & 190 & 0 & 284 & 0 & $16 !$ & 0: & 41 & 0 & 2 & 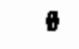 & 32 & & 35 & $\theta$ & 27 & \\
\hline 55 & t & 43 & 0 & 58 & 53 & 6 & 50 & 42 & 6: & 25 & (6) & 2 & 0 & 26 & 24 & 6 & 23 & 9 & \\
\hline$\theta$ & 5 & & 6 & 57 & 53 & 0 & 50 & 42 & : : & 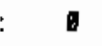 & 26 & & 0 & 2 & & & 23 & & \\
\hline 0 & 52 & 4 & 50 & 53 & 1 & 50 & 49 & 177 & 8: & 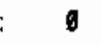 & 24 & & 23 & 25 & & & 3 & & \\
\hline 0 & 53 & 41 & 0 & 53 & 60 & 48 & b & 41 & $177:$ & 0 & 25 & 19 & $\$$ & 25 & 23 & 22 & 0 & 19 & 82 \\
\hline 52 & 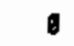 & 46 & 49 & D & 58 & $4 B$ & 49 & 177 & $\theta$ & 24 & 0 & 19 & 23 & $\theta$ & 23 & 22 & 23 & 82 & \\
\hline 0 & 54 & 117 & & 6 & 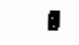 & 6 & 0 & 9 & 0 : & 0 & 27 & 1 & 9 & $\theta$ & & 8 & 0 & B & 0 \\
\hline 59 & & & 5 & 1 & 57 & 204 & 1 & 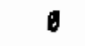 & : & : & & & & & & & & & \\
\hline 56 & 68 & 43 & 54 & 58 & 54 & 53 & 54 & 45 & $45:$ & 24 & 24 & 19 & 24 & 24 & 24 & n & 24 & & $a$ \\
\hline 55 & 38 & 41 & 5 & 43 & 46 & 48 & 38 & 40 & 42: & 24 & 17 & 18 & 24 & 19 & 20 & 21 & 18 & 7 & 18 \\
\hline 58 & 49 & & 5 & 45 & 48 & 4 & 45 & 39 & 41: & 24 & 21 & 4 & 23 & 28 & & 11 & 20 & 7 & 18 \\
\hline 20 & & 158 & & 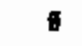 & 189 & & 0 & 158 & $784:$ & : & 0 & 20 & 34 & & 34 & & & 8 & 35 \\
\hline 188 & & & & 189 & $\theta$ & & & 784 & 6 & & 33 & & 32 & 34 & & & 31 & & \\
\hline 214 & 146 & 1 & 208 & 181 & 6 & 196 & 137 & b & 150 & 39 & 27 & 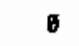 & 37 & 29 & 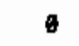 & 34 & 25 & & 27 \\
\hline 285 & & & & $\theta$ & 232 & 5 & 6 & 211 & 6 & 52 & & 30 & 48 & 6 & 42 & 10 & ฮ & 36 & \\
\hline D & & & . & 169 & $\theta$ & 158 & 586 & 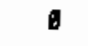 & (1: & 9 & 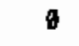 & & $\theta$ & 30 & & 28 & 163 & & \\
\hline 29 & $2 B$ & & 25 & 29 & 121 & 0 & 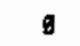 & $B$ & a & 19 & 10 & & 9 & 19 & & $a$ & & & \\
\hline 28 & 2 & 2 & 2 & 28 & 2 & 25 & 121 & $\theta$ & 8 & 10 & 10 & & 9 & 10 & & 9 & 43 & 8 & \\
\hline 38 & 35 & 27 & 33 & 38 & 33 & 33 & 33 & 128 & $\theta$ & 13 & 12 & 16 & 12 & 13 & 2 & 12 & 12 & 3 & \\
\hline 0 & 35 & 27 & 32 & 35 & 33 & 33 & 33 & $2 B$ & 128 & 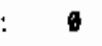 & 12 & 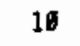 & 11 & 22 & 16 & 12 & 12 & 10 & 43 \\
\hline 0 & 139 & 108 & 8 & 140 & 134 & 6 & 132 & 712 & $\theta$ & 1 & 25 & 19 & 0 & 25 & 24 & 0 & 23 & 128 & \\
\hline 146 & 139 & D & 137 & 138 & 8 & 133 & 133 & 724 & 0 & 25 & 24 & 1 & 24 & 24 & 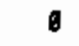 & 23 & 23 & $12 B$ & \\
\hline 51 & 54 & 9 & 51 & 50 & $\theta$ & 50 & 51 & 0 & 41 & 24 & 25 & a & 24 & 23 & 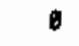 & 23 & 24 & $B$ & 19 \\
\hline 52 & 42 & 0 & 51 & 42 & 50 & 157 & 8 & 0 & 6 & 21 & 18 & 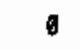 & 21 & 18 & $2 x$ & 87 & 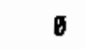 & 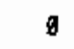 & \\
\hline 182 & 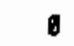 & 143 & 175 & 0 & 173 & 171 & 0 & 142 & 146 & 34 & $g$ & 28 & 32 & $a$ & 32 & 32 & 0 & 28 & 27 \\
\hline 73 & 73 & $\theta$ & 71 & 74 & $\theta$ & 88 & 69 & 0 & 58 & 34 & 34 & 6 & 33 & 34 & 0 & 31 & 33 & $\theta$ & 27 \\
\hline 0 & 74 & 57 & 0 & 74 & 78 & g & 69 & 58 & 0 & 6 & 34 & 28 & 6 & 34 & 32 & $\theta$ & 32 & 27 & \\
\hline 48 & D & 38 & 57 & $\theta$ & 0 & 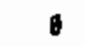 & 0 & 0 & 0 & 17 & $a$ & 13 & 58 & 0 & 6 & $y$ & 0 & g & \\
\hline
\end{tabular}


TABLE A.3. Upper Reference Case, 1985 Inventory and Projected Annual Reactor Discharges (contd)

ASSERIES

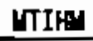

REACTOR

SAN ONOFRE 2

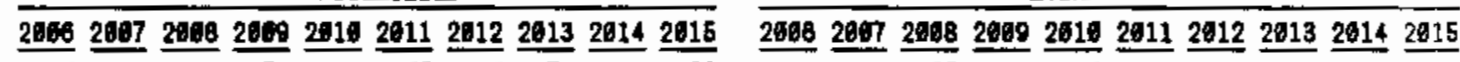

SAN QKOFRE 3

SEABRODK 1

SEABRODK 2

SEQLOYAH I

SEQUOYAH 2

SHOREHW

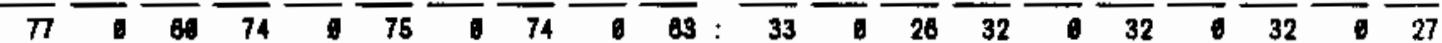

SOUTH TEXS, UNIT 1

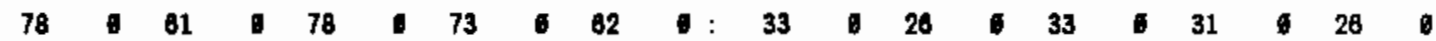

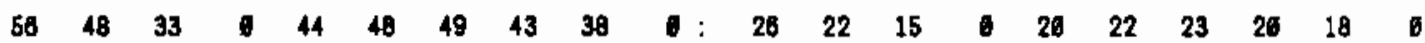

$\begin{array}{llllllllllllllllllllllll} & 54 & 48 & 61 & 54 & 58 & 0 & 52 & 55 & 57 & 0 & 25 & 22 & 28 & 25 & 27 & 0 & 24 & 25 & 28\end{array}$

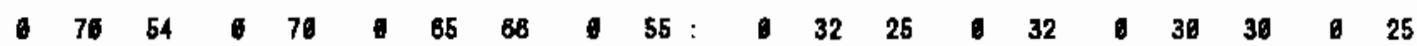

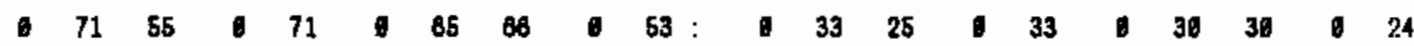

$\begin{array}{lllllllllllllllllllll}46 & 30 & 31 & 44 & 34 & 36 & 39 & 29 & 30 & 33 & : & 25 & 16 & 17 & 24 & 18 & 19 & 21 & 18 & 18 & 58\end{array}$

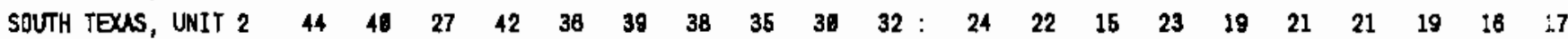

ST LUCIE 1

ST LUCIE 2

SUMER :

SURRY 1

SURRY 2

SUSQUERANNA 1

SUSQUEHANNA 2

3 VILE ISL 1

TROJAN

TURKEY PT 3

TURKEY PT 4

VOGILE 1

VOGTLE 2

VT YANKEE :

$\begin{array}{lllllllllllllllllll}68 & 51 & 61 & 6 & 82 & 81 & 49 & 53 & 25 & 19 & 19 & 23 & 0 & 24 & 23 & 0 & 19 & 26\end{array}$

$\begin{array}{llllllllllllllllllll}63 & 48 & 63 & 69 & 6 & 59 & 49 & 0 & 0 & 0 & 25 & 19 & 9 & 25 & 23 & 0 & 23 & 19 & 0\end{array}$

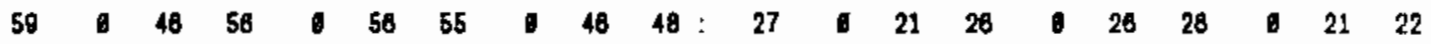

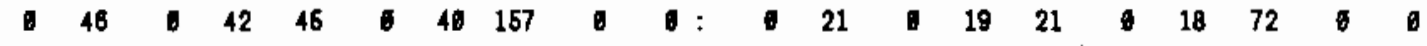

$\begin{array}{lllllllllllllllllll}46 & 35 & 42 & 4 & 4 & 46 & 158 & 0 & 0 & 21 & 0 & 16 & 19 & 0 & 20 & 18 & 72 & 0 & 0\end{array}$

$\begin{array}{llllllllllllllllllll} & 195 & 154 & 195 & 386 & 185 & 185 & 153 & 0 & 0 & 34 & 27 & 9 & 34 & 32 & 0 & 32 & 28 & 8\end{array}$

$\begin{array}{llllllllllllllllllll}197 & 154 & 191 & 186 & 186 & 153 & 158 & 34 & 34 & 153 & 27 & 33 & 0 & 32 & 32 & & 28 & 27\end{array}$

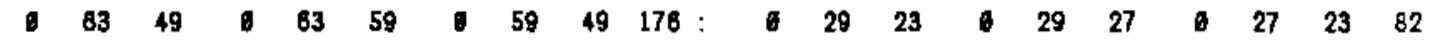

$\begin{array}{lllllllllllllllllllll}42 & 42 & 32 & 48 & 42 & 40 & 48 & 46 & 33 & 34: & 19 & 19 & 15 & 18 & 19 & 18 & 18 & 18 & 15 & 18\end{array}$

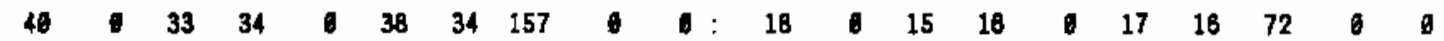

$\begin{array}{llllllllllllllllllll}42 & 42 & 3 & 36 & 41 & 36 & 38 & 157 & 0 & 0 & 19 & 19 & 0 & 17 & 19 & & 17 & 17 & 72 & 6\end{array}$

$\begin{array}{llllllllllllllllllll} & 65 & 51 & 5 & 58 & 59 & 5 & 57 & 52 & 0 & 0 & 39 & 24 & 0 & 27 & 27 & 0 & 28 & 24 & 0\end{array}$

WASH NUCLEAR 2

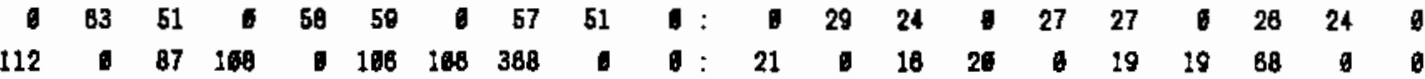

IATERFORD 3

WATTS BAR I

WATTS BAR 2

INP-1

NNP-3

IOLF CREEX 1

YANKEE-RONE :

ZION 1

ZION 2

GRUASIICK 1 PNR

BRUASTICK 2 PWR

HARRIS 1 GUR POOL

MORRIS-GUR

NORRIS-PNR

WEST YNEY-B

VEST YALEY-P

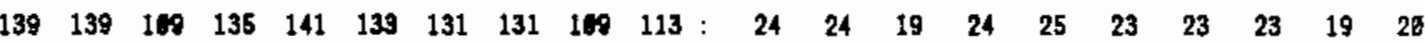

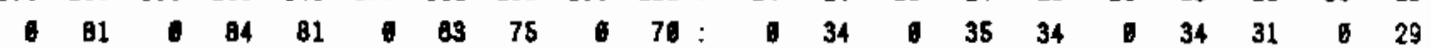

$\begin{array}{lllllllllllllllllllll}65 & 61 & 0 & 69 & 53 & 59 & 59 & 56 & 49 & 36 & 28 & 0 & 32 & 24 & 0 & 27 & 0 & 26 & 23\end{array}$

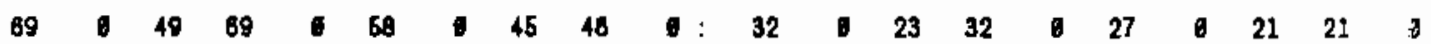

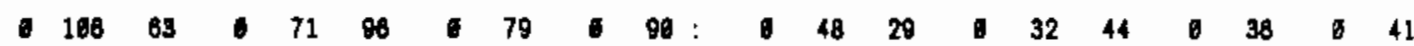

$\begin{array}{lllllllllllllllllllll}98 & 82 & 43 & 89 & 48 & 68 & 87 & 52 & 54 & 62 & : & 43 & 36 & 19 & 36 & 20 & 36 & 29 & 23 & 24 & 27\end{array}$

$\begin{array}{lllllllllllllllllllll}42 & 42 & 32 & 46 & 42 & 40 & 39 & 40 & 33 & 34 & 19 & 19 & 19 & 15 & 19 & 19 & 19 & 18 & 19 & 15 & 13\end{array}$

BUR GENERTC

PWR GENERIC

TOTNL GENERIC

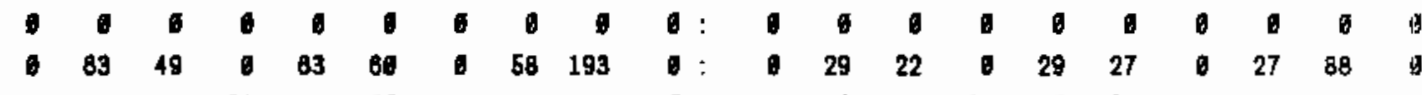

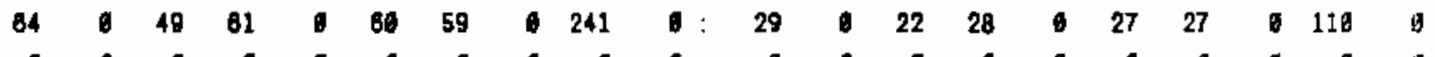

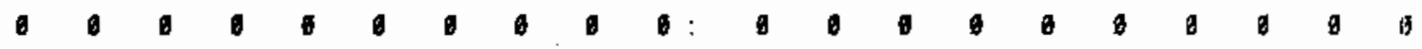

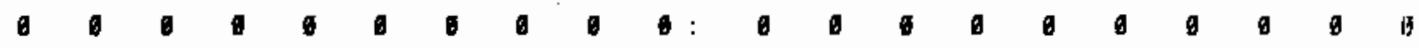

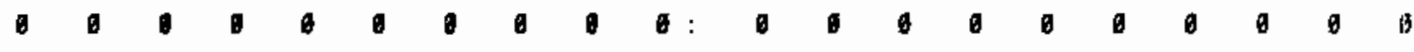

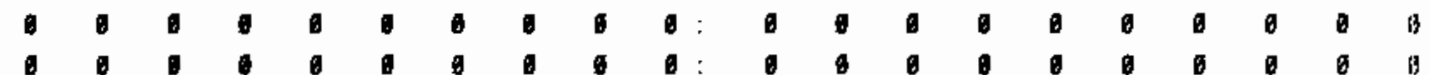

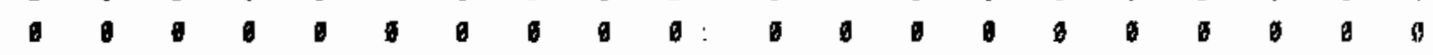

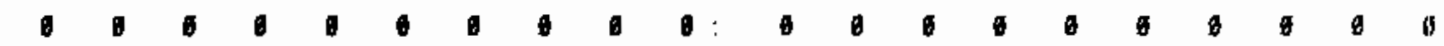
$\begin{array}{lllllllllllllllllllll}1120 & 781 & 1601 & 1989 & 1464 & 1754 & 2818 & 2384 & 2883 & 3588 & 285 & 143 & 183 & 358 & 254 & 319 & 474 & 436 & 523 & 655\end{array}$ $\begin{array}{lllllllllllllllllllll}713 & 891 & 816 & 1251 & 1373 & 1441 & 1901 & 2117 & 2273 & 2870 & : & 325 & 360 & 364 & 554 & 694 & 633 & 832 & 918 & 995 & 1250\end{array}$ $\begin{array}{llllllllllllllllllll}1833 & 1582 & 1817 & 3231 & 2777 & 3195 & 4519 & 4591 & 5158 & 8458 & 539 & 563 & 547 & 912 & 858 & 952 & 1386 & 1354 & 1518 & 1965\end{array}$ 
TABLE A.3. Upper Reference Case, 1985 Inventory and Projected Annual Reactor Discharges (contd)

\begin{tabular}{|c|c|c|c|c|c|c|c|c|c|c|c|}
\hline \multirow[b]{2}{*}{ REACTOR } & \multicolumn{5}{|c|}{ 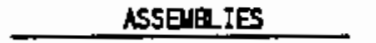 } & & \multicolumn{5}{|c|}{ जIHW } \\
\hline & 2018 & 2017 & 2818 & 2619 & 2028 & & 2016 & 2017 & 2018 & 2019 & 2828 \\
\hline ARK MOEAR 1 & 6 & 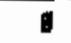 & 0 & 8 & 6 & & $\theta$ & $\theta$ & 0 & 0 & . \\
\hline ARX MCLEAR 2 & 1 & 55 & 57 & - & 56 & : & 6 & 23 & 24 & 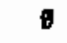 & 23 \\
\hline B YNШEY 1 & 63 & 157 & $\theta$ & 6 & a & : & 24 & 72 & 9 & 0 & G \\
\hline B YNШEY 2 & 47 & 51 & 0 & 38 & 52 & : & 22 & 24 & 6 & 18 & 24 \\
\hline B日乚EONTE 1 & 85 & 0 & 72 & 83 & $\theta$ & : & 30 & 0 & 33 & 29 & 0 \\
\hline B日—EONTE 2 & 54 & E & 68 & 82 & 6 & : & 25 & $\theta$ & 31 & 28 & 0 \\
\hline BIS ROCK 1 & $\theta$ & 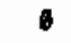 & B & 0 & y & : & $\theta$ & 6 & $\theta$ & 8 & g \\
\hline BRAIDTO0D 1 & 52 & 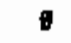 & 80 & 44 & a & : & 22 & b & 25 & 19 & 9 \\
\hline BRAIOTO00 2 & 52 & $\theta$ & 84 & 43 & $\theta$ & : & 22 & 0 & 27 & 18 & b \\
\hline BRDNAS FERRY1 & 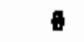 & - & & - & 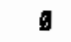 & : & 0 & $\theta$ & $B$ & 0 & 0 \\
\hline BROTHS FERRY2 & 0 & 9 & ? & $\theta$ & 6 & : & 0 & $\theta$ & 0 & 8 & 0 \\
\hline BRONIS FERRY3 & 184 & 784 & 0 & 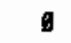 & 0 & : & 30 & 139 & $\theta$ & 6 & $g$ \\
\hline BhussIICX 1 & 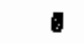 & $\bullet$ & ฮ & 6 & 0 & & a & 6 & g & 0 & 6 \\
\hline 8RUNSTICX 2 & $\theta$ & ] & 6 & - & 0 & : & 0 & $\theta$ & 0 & 0 & 0 \\
\hline BYRON 1 & B & 88 & 71 & 8 & 67 & : & 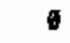 & 28 & 30 & 6 & 28 \\
\hline BYRON 2 & 52 & 68 & D & 44 & 59 & : & 22 & 25 & 0 & 18 & 25 \\
\hline CALANAY I & 87 & 89 & a & 69 & 71 & : & 28 & 29 & 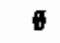 & 25 & 38 \\
\hline CNVERT CLF 1 & 0 & - & o & 0 & 6 & : & 6 & 0 & D & 0 & 9 \\
\hline CNYYERT CLF 2 & a & 72 & 216 & - & 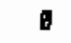 & : & a & 27 & B2 & D & 8 \\
\hline CATATBA 1 & 47 & 0 & 55 & 41 & 51 & : & 29 & $\theta$ & 23 & 17 & 22 \\
\hline CATAWBA 2 & 40 & 48 & a & 34 & 45 & : & 17 & 29 & 0 & 14 & 19 \\
\hline CLIMTON 1 & 142 & $\theta$ & 169 & 138 & $\theta$ & : & 28 & 0 & 29 & 25 & 0 \\
\hline COMANCHE PK 1 & 43 & 48 & 40 & 42 & 40 & : & 17 & 19 & 18 & 17 & 19 \\
\hline COMAHCHE PK 2 & 40 & 47 & 49 & 34 & 47 & : & 16 & 19 & 20 & 14 & is \\
\hline $\operatorname{coo} 1$ & - & 0 & $\theta$ & 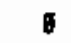 & 0 & : & $\theta$ & 0 & 6 & $\theta$ & 0 \\
\hline cook 2 & g & 69 & 74 & 193 & च & ; & g & 28 & 30 & 78 & 0 \\
\hline COOPER STN & 0 & 0 & - & 6 & 0 & : & $\theta$ & 4 & $\theta$ & 0 & 甲 \\
\hline CRYSTN RVR 3 & 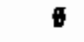 & 65 & 177 & 0 & g & : & 0 & 25 & 82 & g & 6 \\
\hline DAVIS-BESSE 1 & o & 47 & 51 & 178 & $B$ & : & ( & 22 & 24 & 63 & 0 \\
\hline DIAE-O CANYOH-1 & - & 81 & - & 71 & - & : & 0 & 37 & 6 & 33 & $\#$ \\
\hline DIARQO CANYON-2 & 77 & 1 & 89 & - & 87 & ; & 35 & 0 & 41 & 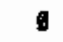 & 40 \\
\hline DRESDEN 1 & a & 6 & g & 0 & $\theta$ & : & 0 & t & $\theta$ & (1) & 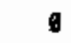 \\
\hline DRESOEY 2 & - & D & 6 & o & 0 & : & g & a & t & a & B \\
\hline DRESDEN 3 & 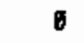 & 9 & o & a & $\exists$ & : & $\theta$ & 9 & a & 8 & 0 \\
\hline DUANE ARNDLD & 388 & - & $\theta$ & 8 & $\theta$ & ; & 04 & 0 & g & E & $B$ \\
\hline ENRICD FERNI-2 & 212 & 229 & 9 & 198 & - & : & 39 & 42 & 6 & 36 & 0 \\
\hline FAREEY 1 & 52 & 157 & $\theta$ & 0 & $\theta$ & & 24 & 72 & $\theta$ & $\sigma$ & $\theta$ \\
\hline FARLEY 2 & 52 & 54 & $\theta$ & 48 & 55 & : & 24 & 25 & 9 & 21 & 25 \\
\hline FITZPATRICK & 580 & 0 & $g$ & 6 & 9 & ; & 99 & $\theta$ & (6) & 0 & 9 \\
\hline FORT CNLHOUN & B & 0 & $\theta$ & - & $\theta$ & : & 8 & g & (1) & b & 8 \\
\hline GINKA & B & 0 & $\theta$ & $\theta$ & a & : & 8 & 8 & 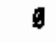 & $\theta$ & b \\
\hline CRAND aUf 1 & 189 & 287 & 0 & 172 & 212 & : & 33 & 38 & $B$ & 30 & 37 \\
\hline GRAND GLF 2 & 6 & 235 & 233 & $\theta$ & 245 & : & 0 & 41 & 41 & $\theta$ & 43 \\
\hline HADON NECK & 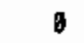 & 0 & g & 0 & g & : & g & 0 & B & 8 & 9 \\
\hline HARRIS 1 & o & 36 & 39 & $\theta$ & 37 & & 6 & 17 & 14 & 6 & 17 \\
\hline HATCH I & $\theta$ & 8 & 0 & 9 & $B$ & : & $\theta$ & 0 & 9 & 0 & $b$ \\
\hline HATCH 2 & 132 & 145 & 6 & 126 & 580 & : & 25 & 27 & 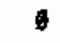 & 22 & 104 \\
\hline HOPE CREEX & 184 & 183 & 9 & 139 & 187 & & 30 & 33 & b & 25 & 34 \\
\hline
\end{tabular}


TABLE A.3. Upper Reference Case, 1985 Inventory and Projected Annual Reactor Discharges (contd)

\begin{tabular}{|c|c|c|c|c|c|c|c|c|c|c|c|}
\hline \multirow[b]{2}{*}{ REACTOR } & \multicolumn{5}{|c|}{ ASSERLIES } & & \multicolumn{5}{|c|}{ NTIHU } \\
\hline & $\underline{2616}$ & 2017 & 2918 & 2019 & $\underline{2020}$ & & $\underline{2016}$ & 2017 & 2018 & 2619 & $\underline{2920}$ \\
\hline HNBOLDT BAY & 0 & $\theta$ & 6 & 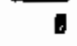 & 0 & & 0 & 6 & 6 & 6 & 8 \\
\hline IMDIAN PT 1 & $\boldsymbol{t}$ & 6 & 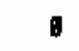 & $\theta$ & 6 & & - & 0 & 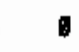 & $a$ & $\theta$ \\
\hline INOIAN PT 2 & 0 & - & 0 & 6 & 0 & & 6 & 0 & 0 & 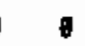 & $\theta$ \\
\hline INDIAN PT 3 & 193 & o & 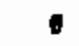 & 0 & $\theta$ & & $8 B$ & 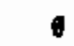 & $\boldsymbol{\theta}$ & 6 & 6 \\
\hline KEAUNEE & 0 & 6 & 6 & 0 & 日 & & 6 & 6 & $\theta$ & $a$ & $\theta$ \\
\hline LACROSSE & g & 0 & - & - & 6 & & - & 6 & 0 & 1 & $\theta$ \\
\hline LASA—E CTY 1 & 158 & 173 & - & 142 & 174 & & 29 & 31 & - & 28 & 32 \\
\hline LASALE CTY 2 & 157 & 1 & 188 & 141 & 0 & : & 29 & $\theta$ & 34 & 28 & 0 \\
\hline LIMERICK 1 & 154 & 109 & - & 140 & 172 & : & 27 & 30 & $\theta$ & 25 & 30 \\
\hline LIMERICK 2 & $\theta$ & 198 & 0 & 179 & 221 & & 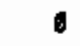 & 35 & 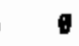 & 32 & 39 \\
\hline MINE YANKEE & a & 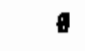 & $a$ & 1 & 0 & & 0 & 6 & 6 & $b$ & 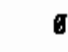 \\
\hline ICCUIRE 1 & 1 & 58 & 53 & 41 & 6 & : & 6 & 21 & 22 & 17 & 0 \\
\hline UCCUIRE 2 & 48 & 50 & a & 41 & 51 & : & 19 & 21 & $\theta$ & 17 & 22 \\
\hline HIDLAKD-1 & $2 B$ & 28 & 29 & 26 & 28 & : & 9 & 9 & 10 & 8 & 9 \\
\hline UTOLANO-2 & 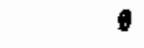 & 53 & 54 & 6 & 53 & . & 6 & 25 & 25 & - & 25 \\
\hline UILLSTONE 1 & $\theta$ & 0 & $\theta$ & 6 & 0 & : & $\theta$ & 0 & 0 & $\theta$ & $\theta$ \\
\hline MUUSTONE 2 & 217 & : & 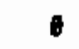 & $\theta$ & $\theta$ & t & 88 & $\theta$ & $\boldsymbol{\theta}$ & 6 & 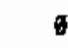 \\
\hline LIШSTONE 3 & 52 & 62 & 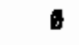 & 43 & 58 & : & 24 & 29 & 0 & 26 & 27 \\
\hline 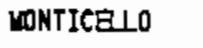 & 1 & 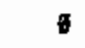 & 0 & g & 0 & t & $\theta$ & 6 & 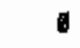 & 0 & $\theta$ \\
\hline NINE UILE PT1 & - & 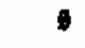 & - & $\theta$ & $\varpi$ & : & & $\theta$ & 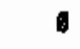 & 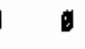 & $\theta$ \\
\hline MINE MILE PT2 & 168 & $\theta$ & 268 & B & 193 & : & 29 & $\theta$ & 35 & - & 33 \\
\hline NORTH AXNA I & 44 & 48 & 158 & 6 & 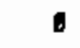 & ; & 2ब & 22 & 72 & 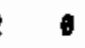 & $\theta$ \\
\hline NBRTH ANMA 2 & 44 & 48 & 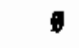 & 30 & 51 & : & 20 & 22 & 6 & 18 & 24 \\
\hline DCONEE 1 & $\theta$ & - & 6 & - & $\theta$ & : & $\theta$ & $\theta$ & 0 & 6 & a \\
\hline OCONEE 2 & 6 & $\bullet$ & $\theta$ & $\theta$ & o & : & 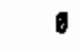 & ต & 6 & - & - \\
\hline OCONEE 3 & 0 & 6 & 1 & a & - & : & 6 & 6 & 0 & 1 & $\theta$ \\
\hline DYSTER CRK I & 0 & 6 & - & b & 8 & : & 6 & 0 & 6 & 0 & 8 \\
\hline PALISADES & $\theta$ & - & $\theta$ & 6 & 0 & : & 8 & - & 0 & 9 & $\theta$ \\
\hline PNO VEROE 1 & 47 & 51 & 54 & 41 & 51 & : & 21 & 22 & 24 & 18 & 22 \\
\hline PNLO VERDE 2 & 44 & 47 & 37 & 41 & 45 & : & 19 & 21 & 16 & 18 & 20 \\
\hline PALO VERDE 3 & 40 & 45 & 48 & 35 & 45 & : & 17 & 20 & 21 & 15 & 20 \\
\hline PEACHBOTTOU 2 & 6 & 6 & च & $\theta$ & 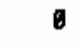 & : & $g$ & - & $\theta$ & 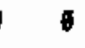 & $\theta$ \\
\hline PEACHBOTTON 3 & - & $\theta$ & 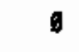 & 0 & 6 & : & $\theta$ & 0 & 0 & $\theta$ & 0 \\
\hline PERRY 1 & 0 & 172 & 188 & 6 & 176 & : & 6 & 31 & 34 & 0 & 32 \\
\hline PERRY 2 & 218 & 224 & - & 203 & 293 & : & 40 & 41 & 0 & 37 & 42 \\
\hline PILGRIU 1 & 0 & 6 & 0 & - & 6 & : & 0 & $\theta$ & 1 & 6 & 6 \\
\hline POINT BEACH 1 & 6 & 9 & $\theta$ & $\theta$ & $\theta$ & & $\theta$ & $\theta$ & $\theta$ & $\theta$ & $\theta$ \\
\hline POINT BEACH 2 & $\theta$ & 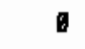 & $\$$ & 0 & $\theta$ & : & $\theta$ & 0 & y & 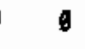 & $\theta$ \\
\hline PRAIRIE ISL 1 & $\theta$ & 6 & 0 & 0 & 9 & : & $\theta$ & $\theta$ & 0 & 6 & 0 \\
\hline PQAIRIE ISL 2 & 6 & 6 & - & 9 & 6 & & б & 6 & 0 & $\theta$ & $\theta$ \\
\hline QUAD CITIES 1 & 9 & - & 6 & $\theta$ & a & & $\theta$ & 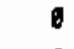 & 6 & 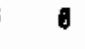 & $\theta$ \\
\hline QUAD CITIES 2 & $\theta$ & 0 & 0 & 6 & 6 & : & 0 & $\theta$ & $\theta$ & 6 & $\theta$ \\
\hline RANCHO SECO-1 & 177 & 6 & a & 8 & $\theta$ & $\cdot$ & 82 & $\theta$ & 0 & g & 9 \\
\hline RCBINSOH 2 & $\theta$ & 1 & 8 & 0 & 0 & 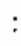 & 0 & 6 & ø & $a$ & 9 \\
\hline RVR BEND 1 & 0 & 183 & 178 & 6 & 187 & & $\theta$ & 30 & 33 & $\theta$ & 31 \\
\hline SALE UNIT 2 & 83 & 0 & 71 & 55 & $\theta$ & ${ }^{\circ}$ & 28 & $\theta$ & 33 & 25 & 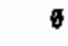 \\
\hline SNEE 1 & 80 & 68 & 193 & $\theta$ & 6 & ${ }^{\circ}$ & 28 & 38 & 89 & 8 & $\theta$ \\
\hline SAN ONOFRE 1 & 6 & $\theta$ & - & 8 & $\theta$ & t & $\theta$ & $\theta$ & 6 & 6 & \\
\hline
\end{tabular}


TABLE A.3. Upper Reference Case, 1985 Inventory and Projected Annual Reactor Discharges (contd)

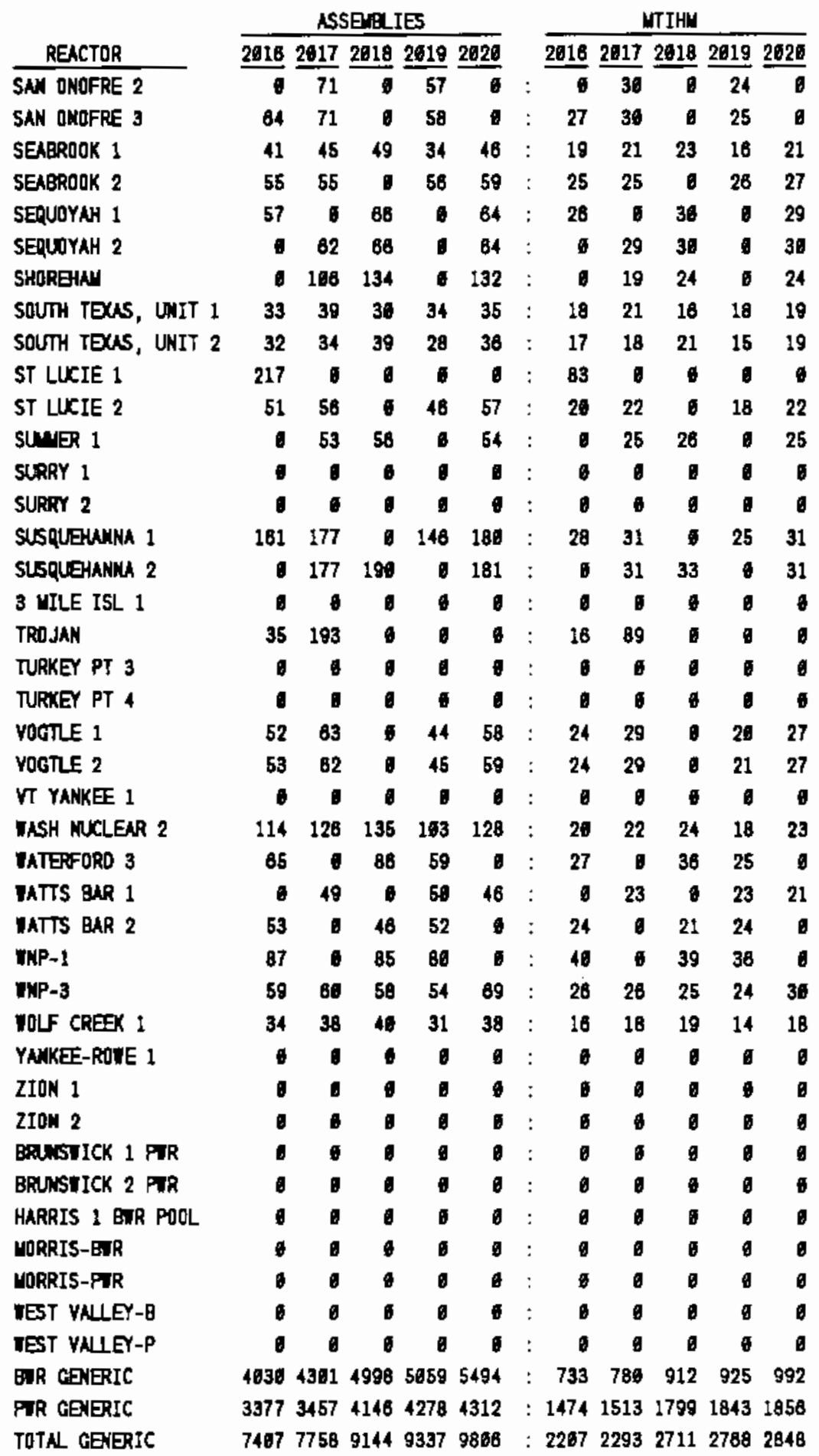


TABLE A.3. Upper Reference Case, 1985 Inventory and Projected Annual Reactor Discharges (contd)

\begin{tabular}{|c|c|c|c|c|c|c|c|c|c|c|c|}
\hline & & & & & & SUBTOTAN & BY REAC & TYPE A & TOTALS & & \\
\hline & & $\underline{1985}$ & $\underline{1986}$ & $\underline{1987}$ & $\underline{1988}$ & $\underline{1989}$ & $\underline{1990}$ & $\underline{1991}$ & $\underline{1992}$ & 1993 & 1994 \\
\hline$P$ R & ASSEMBLY & 17862 & 2288 & 2315 & 2793 & 3116 & 3158 & 2958 & 3282 & 3114 & 2763 \\
\hline$P \mathbb{R}$ & WTIH & 7558 & 981 & 1606 & 1192 & 1355 & 1972 & 1277 & 1438 & 1354 & 1176 \\
\hline BWR & ASSEURLY & 28933 & 2342 & 3016 & 3581 & 4121 & 3478 & 4017 & 4284 & $38: 2$ & 4607 \\
\hline 8WR & KTIHM & 5695 & 419 & 548 & 848 & 744 & 828 & 723 & 782 & 848 & 829 \\
\hline TOTAL & ASSEIBLYY & 45895 & 4688 & 5331 & 6374 & 7237 & 8832 & 8973 & 7528 & 8716 & 7310 \\
\hline TOTAL & MIHU & 12651 & 1480 & 1548 & 1846 & 2180 & 2080 & 2080 & 2193 & 2000 & 2600 \\
\hline & & $\underline{1998}$ & $\underline{1997}$ & $\underline{1998}$ & $\underline{1999}$ & $\underline{2000}$ & $\underline{2001}$ & $\underline{2692}$ & $\underline{2063}$ & 2064 & $\underline{2645}$ \\
\hline PWR & ASSEARCY & 2837 & 3830 & 3029 & 2936 & 3144 & 3862 & 2827 & 3193 & 3571 & 3431 \\
\hline FWR & MTIHY & 1229 & 1319 & 1368 & 1278 & 1372 & 1347 & 1234 & 1391 & 1575 & 1512 \\
\hline gur & ASSEMBQY & 4304 & 3783 & 3929 & 4032 & 4878 & 3658 & 4277 & 4501 & 4184 & 4271 \\
\hline EXR & МIHU & 771 & 881 & 885 & 722 & $72 B$ & 653 & 768 & 010 & 744 & 788 \\
\hline TOTAL & ASSEMAY & 7141 & 6813 & 8958 & 6962 & 7222 & $671 B$ & 7104 & 7894 & 7755 & 7792 \\
\hline TOTAL & MTIH & 2698 & 2060 & 1991 & 2908 & 2100 & 2060 & 2060 & 2289 & 2319 & 2280 \\
\hline & & $\underline{268 B}$ & $\underline{2067}$ & 2008 & $\underline{2009}$ & $\underline{2010}$ & 2611 & 2012 & 2013 & $\underline{2914}$ & $\underline{2915}$ \\
\hline PWR & ASSEMBLY Y & 3868 & 4318 & 3859 & 4264 & 4824 & 4751 & 5290 & 5871 & 5598 & 8212 \\
\hline PWR & VTIH & 1882 & 1961 & 1698 & 1828 & 2118 & 2058 & 2291 & 2571 & 2472 & 2769 \\
\hline BWR & ASSEIBLY & 5581 & 4484 & 5671 & 7483 & 8972 & 8932 & 7332 & 8236 & 9590 & 8222 \\
\hline EWR & MTIHM & 998 & 799 & 910 & 1274 & 1081 & 1241 & 1389 & 1128 & 1727 & 1491 \\
\hline TOTAL & ASSEABLY & 9247 & 8774 & 8936 & 11287 & 18896 & 11683 & 12622 & 12167 & 15188 & 14434 \\
\hline TOTAL & WTIHN & 2600 & 2760 & 2599 & 3100 & 3199 & 3300 & 3696 & 3899 & 4200 & 4208 \\
\hline
\end{tabular}

\begin{tabular}{|c|c|c|c|c|c|c|}
\hline & & 2918 & 2017 & $\underline{2818}$ & 2919 & 2926 \\
\hline PWR & ASSEMBLY Y & 8210 & 8126 & 6835 & 8359 & 8651 \\
\hline PWR & MIHM & 2718 & 2696 & 2963 & 2752 & 2632 \\
\hline BWR & ASSEIBLY Y & 7692 & 7749 & 6697 & a870 & 8655 \\
\hline BWR & МIHU & 1281 & 1463 & 1197 & 1247 & 1567 \\
\hline TOTAL & ASSEMBLY & 13382 & 13875 & 13242 & 13228 & 14788 \\
\hline TOTAL & MTIHIN & 3999 & 4698 & 1100 & 3999 & 4199 \\
\hline
\end{tabular}


TABLE A.4. Upper Reference Case, 1985 Inventory and Projected Inventory to 2020

REACTOR

ARK NUCLEAR 1

- ark nuelear 2

Q VNDEY 1

8 VNLEY 2

B日LEONTE 1

BELEONTE 2

BIG ROCK 1

BRAIDWOQD 1

BRAIOHODD 2

BROWNS FERRY!

BROWNS FERRY2

BROWNS FERRY3

BRUNSWICK 1

BRUNSIICK 2

BYRON 1

BYRON 2

CNLAIAY 1

CALERT CLF 1

CALYERT CLF 2

CATAluE 1

CATANBA 2

CLINTEN I

CDUANCHE PK 1

COLANCHE PK 2

COOK 1

COOK 2

COOPER STN

CRYSTAL RVR 3

DAYIS-BESSE 1

DIAELO CANYON-1

DIAEL CANYON-2

DRESDEN 1

DRESDEN 2

DRESDEN 3

DANE ARMOLO

ENRICD FERMI-2

FARLEY 1

FARLEY 2

FITZPATRICK

FORT CNLHDUN

GINNA

GRAND GUF 1

GRAND CULF 2

HADOAM NECK

HARRIS I

HATCH 1

HATCH 2

HOPE CREEK

HLMBOLDT BAY

INOIAN PT 1
Assemblies $\frac{19}{378}$

$198519891987 \underline{1988} \frac{1989}{1906} \frac{1991}{1992} \frac{1993}{1984} \underline{1995}$

Inv

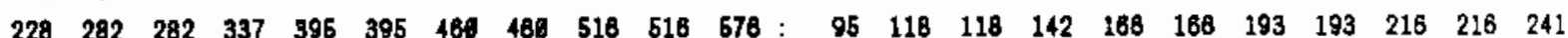
$\begin{array}{lllllllllllllllllllllll}218 & 281 & 397 & 337 & 399 & 486 & 489 & 525 & 586 & 647 & 847 & 166 & 129 & 155 & 156 & 183 & 212 & 212 & 242 & 270 & 298 & 298\end{array}$

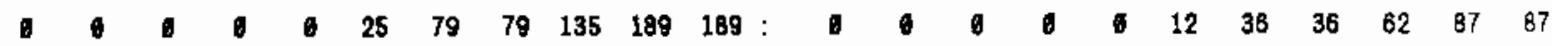

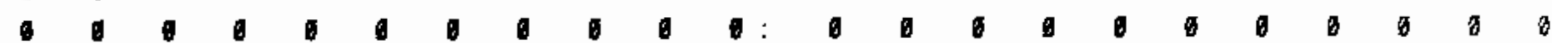
1. $\begin{array}{lllllllllllllllllllllll}192 & 214 & 210 & 224 & 241 & 258 & 277 & 294 & 310 & 310 & 328 & : & 25 & 27 & 27 & 29 & 32 & 34 & 36 & 38 & 41 & 41 & 43\end{array}$ $\begin{array}{llllllllllllllllllll} & 1 & 114 & 114 & 177 & 255 & 255 & 316 & 399 & 0 & 0 & 0 & 0 & 48 & 46 & 75 & 168 & 198 & 134 & 169\end{array}$

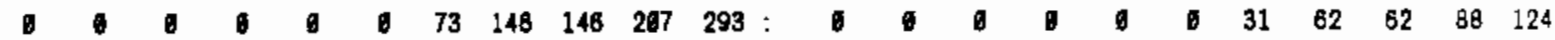
$\begin{array}{llllllllllllllllllllllll}1328 & 1328 & 1328 & 1328 & 1328 & 1328 & 1328 & 1531 & 1531 & 1728 & 1726 & : & 246 & 248 & 246 & 248 & 246 & 246 & 248 & 283 & 283 & 317 & 317\end{array}$

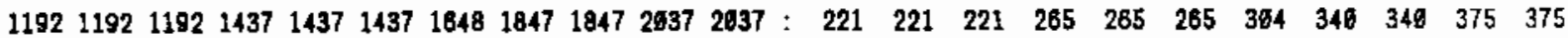

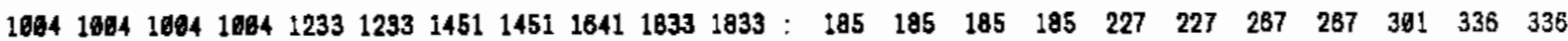
$\begin{array}{lllllllllllllllllllllll}840 & 840 & 989 & 989 & 1142 & 1298 & 1298 & 1481 & 1461 & 1816 & 1779 & 150 & 156 & 184 & 184 & 212 & 241 & 241 & 272 & 272 & 361 & 33 !\end{array}$

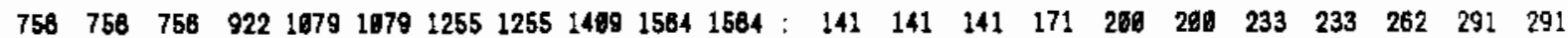

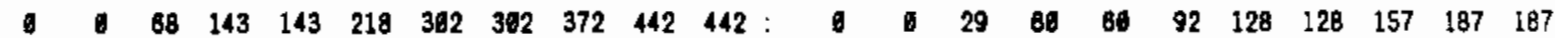
$\begin{array}{lllllllllllllllllllll}1 & 114 & 173 & 173 & 251 & 319 & 319 & 461: & 0 & 0 & 0 & 48 & 73 & 73 & 186 & 135 & 135 & 179\end{array}$

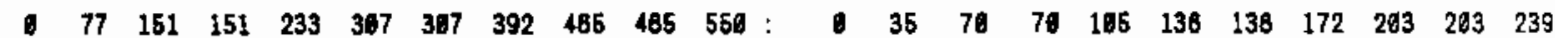
$\begin{array}{lllllllllllllllllllllll}542 & 813 & 813 & 691 & B 91 & 789 & 769 & 851 & 851 & 928 & 928 & 298 & 235 & 235 & 284 & 284 & 294 & 294 & 325 & 325 & 354 & 354\end{array}$ $\begin{array}{lllllllllllllllllllllll}468 & 488 & 534 & 534 & 699 & 669 & 705 & 785 & 782 & 782 & 863 & 179 & 179 & 265 & 265 & 233 & 233 & 289 & 269 & 298 & 298 & 329\end{array}$ $\begin{array}{lllllllllllllllllllllll}8 & 59 & 112 & 188 & 222 & 222 & 283 & 339 & 392 & 392 & 448 & : & 0 & 25 & 47 & 71 & 94 & 94 & 126 & 143 & 186 & 186 & 190\end{array}$

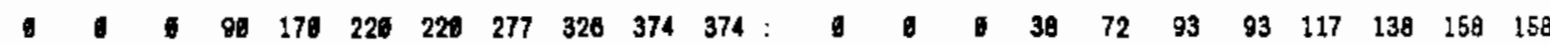

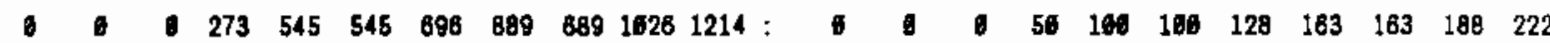
$\begin{array}{lllllllllllllllllllll}0 & 68 & 183 & 212 & 283 & 317 & 386 & 418 & 488: & 0 & 0 & 41 & 75 & 98 & 121 & 143 & 163 & 183 & 284\end{array}$

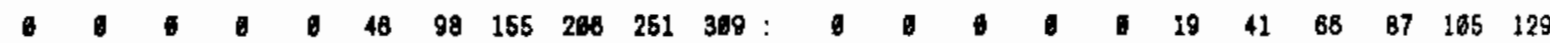
$\begin{array}{lllllllllllllllllllllll}546 & 546 & 665 & 672 & 672 & 749 & 746 & 811 & 876 & 878 & 948 & : & 238 & 238 & 265 & 298 & 296 & 327 & 327 & 368 & 390 & 399 & 423\end{array}$ $\begin{array}{lllllllllllllllllllllll}335 & 415 & 483 & 483 & 558 & 558 & 642 & 720 & 729 & 793 & 971 & : & 154 & 187 & 215 & 215 & 245 & 245 & 279 & 319 & 316 & 340 & 371\end{array}$

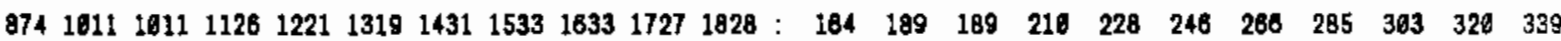
$\begin{array}{lllllllllllllllllllllll}398 & 388 & 375 & 375 & 440 & 446 & 569 & 589 & 569 & 589 & 832 & : & 142 & 142 & 174 & 174 & 204 & 294 & 236 & 236 & 264 & 284 & 293\end{array}$ $\begin{array}{lllllllllllllllllllllll}197 & 197 & 240 & 298 & 298 & 350 & 480 & 489 & 480 & 511 & 511 & : & 93 & 93 & 118 & 146 & 140 & 165 & 192 & 192 & 216 & 240 & 240\end{array}$ $\begin{array}{lllllllllllllllllllllll} & 63 & 63 & 133 & 221 & 221 & 325 & 325 & 412 & 412 & 507 & 0 & 0 & 29 & 29 & 61 & 162 & 102 & 149 & 149 & 189 & 189 & 233\end{array}$

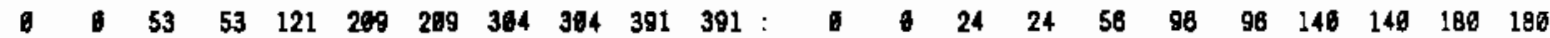
$\begin{array}{lllllllllllllllllllllll}683 & 683 & 693 & 683 & 883 & 683 & 883 & 683 & 603 & 683 & 683 & : & 70 & 70 & 70 & 70 & 70 & 70 & 70 & 70 & 70 & 70 & 70\end{array}$

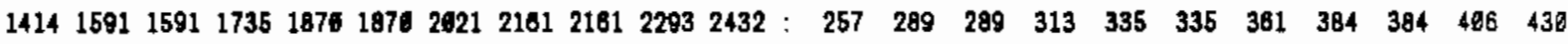

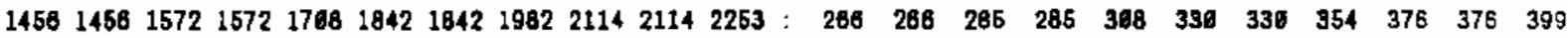
$\begin{array}{lllllllllllllllllllllll}896 & 696 & 795 & 899 & 899 & 1091 & 1123 & 1123 & 1222 & 1322 & 1322 & : & 129 & 129 & 148 & 187 & 187 & 185 & 297 & 297 & 225 & 242 & 242\end{array}$

$\begin{array}{llllllllllllllllllllll} & 0 & 291 & 450 & 456 & 717 & 717 & 964 & 984 & 1221 & 0 & 0 & 0 & 0 & 37 & 83 & 83 & 132 & 131 & 177 & 177 & 223\end{array}$ $\begin{array}{llllllllllllllllllllll}349 & 498 & 498 & 468 & 528 & 528 & 595 & 856 & 658 & 716 & 777 & 160 & 187 & 187 & 215 & 242 & 242 & 274 & 392 & 382 & 339 & 358\end{array}$ $\begin{array}{lllllllllllllllllllllll}188 & 251 & 364 & 384 & 385 & 423 & 423 & 486 & 543 & 543 & 686 & 86 & 115 & 140 & 148 & 188 & 194 & 194 & 224 & 250 & 256 & 279\end{array}$

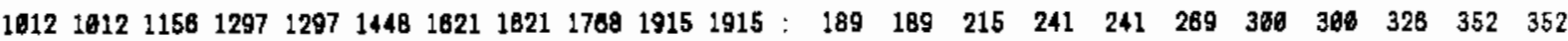
$\begin{array}{lllllllllllllllllllllll}347 & 347 & 381 & 419 & 419 & 458 & 495 & 495 & 531 & 562 & 582 & : & 128 & 128 & 138 & 152 & 152 & 165 & 179 & 179 & 192 & 263 & 203\end{array}$ $\begin{array}{lllllllllllllllllllllll}360 & 390 & 417 & 444 & 471 & 498 & 528 & 556 & 583 & 610 & 838 & : & 199 & 150 & 180 & 170 & 179 & 188 & 198 & 297 & 216 & 225 & 234\end{array}$

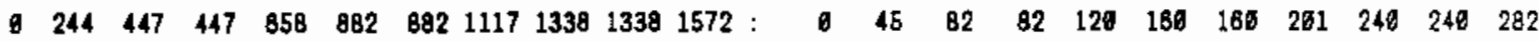

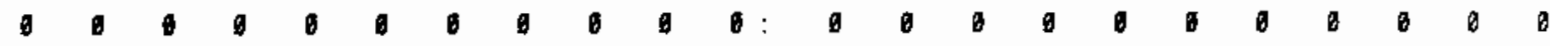
$\begin{array}{lllllllllllllllllllllll}544 & 592 & 833 & 674 & 674 & 715 & 766 & 813 & 813 & 857 & 997 & 224 & 244 & 261 & 278 & 278 & 295 & 316 & 335 & 335 & 353 & 374\end{array}$

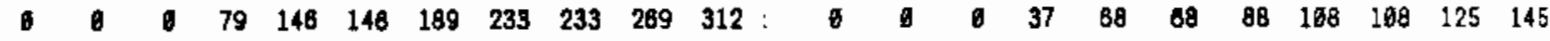

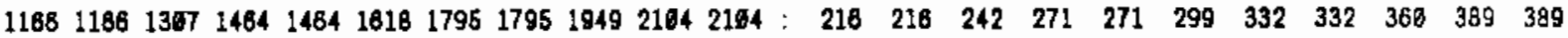
$\begin{array}{lllllllllllllllllllllll}548 & 721 & 880 & 880 & 1615 & 1170 & 1179 & 1333 & 1486 & 1488 & 1648: & 108 & 132 & 158 & 158 & 188 & 215 & 215 & 245 & 274 & 274 & 374\end{array}$ $\begin{array}{llllllllllllllllllll}1 & 324 & 504 & 504 & 741 & 946 & 940 & 1172 & : & 0 & 0 & 9 & 0 & 69 & 93 & 93 & 137 & 173 & 173 & 215\end{array}$

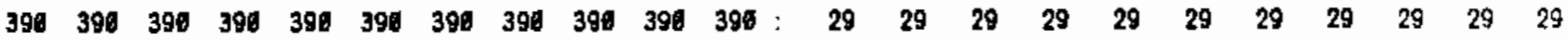

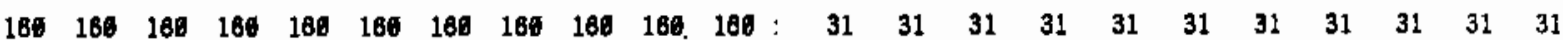


TABLE A.4. Upper Reference Case, 1985 Inventory and Projected Inventory to 2020 (contd)

\begin{tabular}{|c|c|c|c|c|c|c|c|c|c|c|c|c|c|c|c|c|c|c|c|c|c|c|}
\hline \multirow[b]{2}{*}{ REACTOR } & \multirow{2}{*}{$\frac{\operatorname{Inv}}{\underline{1985}}$} & \multicolumn{10}{|c|}{ Assonblies } & \multirow{2}{*}{$\frac{\operatorname{Inv}}{1995}$} & \multicolumn{10}{|c|}{ 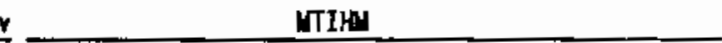 } \\
\hline & & 1986 & $\underline{1987}$ & 1988 & 1989 & $\underline{1990}$ & $\underline{1991}$ & $\underline{1992}$ & 1993 & 1994 & $\underline{1995}$ & & 1986 & 1987 & 2988 & 1989 & 1990 & 1991 & 1992 & 2993 & I994 & 1995 \\
\hline INOIAN PT 2 & 398 & 450 & 510 & 510 & 587 & 624 & 624 & 885 & $\overline{885}$ & 742 & 802 & 179 & 267 & 231 & 231 & 257 & 283 & 283 & 311 & 311 & 336 & 363 \\
\hline INDIAN PT 3 & 292 & 292 & 350 & 415 & 415 & 479 & 479 & 547 & 810 & 816 & 677 & 133 & 133 & 189 & 180 & 190 & 219 & 219 & 256 & 279 & 279 & 369 \\
\hline KEEALWEE & 352 & 377 & 414 & 455 & 488 & 519 & 555 & 588 & 619 & 858 & 693 & 138 & 147 & $1 B 1$ & 177 & 189 & 201 & 215 & 227 & 238 & 251 & 263 \\
\hline LACROSSE & 233 & 281 & 261 & 281 & 281 & 302 & 302 & 323 & 323 & 343 & 343 & 27 & 30 & 30 & 33 & 33 & 37 & 37 & 40 & 40 & 43 & 43 \\
\hline 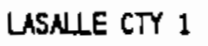 & 132 & 132 & 379 & 379 & 540 & 726 & 726 & 929 & 1104 & 1104 & 1298 & 24 & 24 & 89 & 89 & 99 & 132 & 132 & 188 & 261 & 201 & 237 \\
\hline LASAUE CTY 2 & - & 207 & 287 & 406 & 593 & 593 & 805 & 1000 & 1890 & 1183 & 1377 & 0 & 38 & 38 & 74 & 198 & 108 & 147 & 182 & 182 & 216 & 251 \\
\hline LIUERICK 1 & $\theta$ & 6 & $25 \theta$ & 258 & 437 & 812 & 612 & $B 10$ & $9 B 6$ & 988 & 1177 & 5 & 0 & 46 & 46 & 89 & 112 & 112 & 147 & 179 & 179 & 212 \\
\hline LIMERICK 2 & $\theta$ & 5 & $\bullet$ & 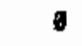 & o & 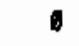 & 0 & 0 & 0 & 269 & 486 & a & $\theta$ & $\boldsymbol{g}$ & $\theta$ & $\oplus$ & 1 & 6 & 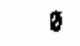 & 9 & 49 & 89 \\
\hline MAINE YANKEE & 787 & 787 & 837 & 800 & 982 & 982 & 1632 & 1032 & 1993 & 1154 & 1154 & 297 & 297 & 318 & 339 & 382 & 382 & 389 & 389 & 412 & 435 & 435 \\
\hline NCOUIRE 1 & 135 & 202 & 254 & 311 & 311 & 368 & 427 & 484 & 484 & 537 & 583 & 62 & 93 & 115 & 139 & 139 & 163 & 189 & 213 & 213 & 235 & 259 \\
\hline WCOUIRE 2 & 48 & 110 & 185 & 225 & 280 & 335 & 335 & 392 & 445 & 499 & 499 & 22 & 51 & 76 & 101 & 125 & 148 & 148 & 172 & 195 & 218 & 218 \\
\hline VIDLAND-1 & $\theta$ & 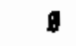 & & 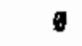 & 6 & . & $\theta$ & 9 & 0 & 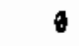 & 0 & 6 & E & $g$ & D & 0 & 0 & $\theta$ & $\theta$ & $\theta$ & 6 & $\theta$ \\
\hline WTDLAND-2 & 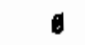 & 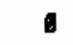 & 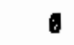 & 0 & $\theta$ & 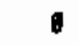 & 6 & a & 6 & 0 & 0 & 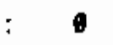 & 6 & - & 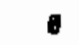 & $\theta$ & 6 & 6 & 8 & $\theta$ & & g \\
\hline NIUSTONE I & 1538 & 1538 & 1899 & 1890 & 1880 & 1880 & 2052 & 2652 & 2219 & 2388 & 2388 & 289 & 289 & 316 & 316 & 346 & 346 & 380 & 380 & 416 & 440 & 448 \\
\hline NILLSTONE 2 & 428 & 480 & 480 & 544 & 6868 & 688 & 889 & 737 & 891 & 885 & $B 85$ & 168 & 187 & 187 & 213 & 239 & 284 & 284 & 292 & 318 & 344 & 344 \\
\hline MIUSTONE 3 & $\theta$ & D & 58 & 58 & 179 & 244 & 244 & 319 & 383 & 383 & 462 & 6 & $\theta$ & 27 & 27 & 83 & 113 & 113 & 147 & 177 & 177 & 213 \\
\hline MONTICELO & 438 & 586 & 641 & 641 & 733 & 825 & 929 & 1625 & 1825 & 1115 & 1218 & 82 & 104 & 110 & 119 & 135 & 150 & 168 & 185 & 185 & 200 & 217 \\
\hline NINE MILE PT1 & 1244 & 1429 & 1429 & 1862 & 1682 & 1764 & 1784 & 1937 & 1937 & 2684 & 2084 & 235 & 288 & 268 & 298 & 298 & 327 & 327 & 357 & 357 & 383 & 383 \\
\hline NINE YILE PT2 & 6 & - & - & d & 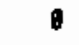 & 206 & 208 & 445 & 445 & 831 & 631 & $\theta$ & 0 & 6 & 0 & 9 & 37 & 37 & 81 & a1 & 115 & 115 \\
\hline NORTH ANNA 1 & 299 & 299 & 349 & 465 & 465 & 460 & 523 & 523 & 577 & 631 & 631 & 137 & 137 & 180 & \pm 88 & 186 & 212 & 241 & 241 & 266 & 291 & 291 \\
\hline NORTH ANNA 2 & 174 & 234 & 278 & 279 & 334 & 389 & 389 & 447 & 501 & 531 & 558 & $B 8$ & 108 & 128 & 128 & 154 & 179 & 178 & 268 & 231 & 231 & 257 \\
\hline OCONEE I & 578 & 833 & 878 & $72 B$ & 728 & 779 & B37 & 896 & 898 & 940 & 993 & 288 & 293 & 314 & 337 & 337 & 381 & 388 & 412 & 12 & 435 & 460 \\
\hline OCONEE 2 & 412 & 487 & 512 & 512 & 583 & 814 & 814 & 687 & 717 & 767 & 787 & 191 & 217 & 237 & 237 & 281 & 285 & 285 & 399 & 332 & 356 & 356 \\
\hline DCONEE 3 & 388 & 388 & 433 & 483 & 534 & 534 & 591 & 844 & 844 & 684 & 747 & 188 & 189 & 201 & 224 & 248 & 248 & 274 & 299 & 299 & 322 & 347 \\
\hline DYSTER CRX 1 & 1204 & 1387 & 1387 & 1514 & 1839 & 1839 & 1781 & 1934 & 1934 & 2978 & 2878 & 227 & 255 & 255 & 281 & 303 & 393 & 328 & 355 & 355 & 381 & 381 \\
\hline PNISADES & 546 & 545 & 598 & 598 & 858 & 714 & 714 & 775 & 775 & $B 32$ & 832 & 219 & 219 & 248 & 240 & 282 & 285 & 285 & 69 & 399 & 331 & 331 \\
\hline PALO YERDE 1 & 6 & 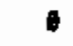 & 47 & 117 & 178 & 288 & 347 & 494 & 457 & 510 & 588 & a & $\sigma$ & 29 & 48 & 73 & 119 & 148 & 171 & 194 & 17 & 41 \\
\hline PNO VERDE 2 & 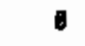 & - & 51 & 177 & 275 & 325 & 373 & 427 & 477 & 525 & 582 & 6 & - & 22 & 73 & 112 & 133 & 154 & 177 & 199 & 220 & 245 \\
\hline PNO VERDE 3 & a & 0 & 6 & I & 78 & 133 & 185 & 242 & 290 & 334 & 397 & $\theta$ & 1 & $\theta$ & 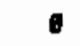 & 32 & 55 & 76 & 160 & 121 & 140 & 108 \\
\hline PEACHBOTTOU 2 & 1464 & 1464 & 1854 & 1854 & 1854 & 2040 & 2269 & 2259 & 2448 & 2638 & 2638 & 272 & 272 & 307 & 344 & 344 & 378 & 417 & 417 & 450 & 484 & 484 \\
\hline PEACHEOTTOL 3 & 1488 & 1498 & 1686 & 1888 & 1858 & 1850 & 2657 & 2249 & 2249 & 2428 & 2616 & 279 & 279 & 316 & 310 & 344 & 344 & 382 & 16 & 16 & 48 & 481 \\
\hline PERRY 1 & 0 & 1 & 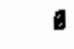 & 346 & 624 & 824 & 802 & 1660 & 1099 & 1187 & 1378 & a & 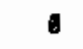 & 8 & 83 & 114 & 114 & 148 & 184 & 184 & 217 & 251 \\
\hline PERRY 2 & 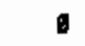 & - & 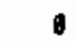 & - & 0 & 6 & 6 & 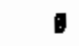 & 6 & 260 & 268 & $\theta$ & 0 & $\theta$ & 6 & $\theta$ & • & 6 & $\sigma$ & $g$ & 38 & 38 \\
\hline PILGRIU 1 & 1128 & 1306 & 1386 & 1475 & 1475 & 1637 & 1837 & 1808 & 1868 & 1973 & 1973 & 213 & 244 & 244 & 274 & 274 & 383 & 393 & 333 & 333 & 362 & 362 \\
\hline POINT BEACH I & 483 & 436 & 488 & 506 & 530 & 558 & 688 & 6t6 & 643 & 670 & 696 & 161 & 175 & 188 & 290 & 211 & 221 & 232 & 241 & 251 & 281 & 270 \\
\hline POINT BEACH 2 & 384 & 418 & 448 & 480 & 510 & 537 & 588 & 596 & 623 & 650 & B78 & 153 & 186 & 179 & 196 & 201 & 210 & 221 & 231 & 241 & 251 & 269 \\
\hline PRAIRIE ISL 1 & 326 & 383 & 414 & 448 & 482 & 516 & 555 & 555 & 588 & 821 & 658 & 126 & 149 & 168 & 173 & 185 & 187 & 211 & 211 & 222 & 234 & 246 \\
\hline PRAIRIE ISL 2 & 374 & 411 & 411 & 444 & 477 & 511 & 549 & 584 & 617 & 850 & 685 & 146 & 160 & 160 & 171 & 183 & 195 & 208 & 221 & 233 & 244 & 257 \\
\hline QUAD CITIES 1 & 1289 & 1380 & 1511 & 1511 & 1648 & 1784 & 1784 & 1926 & 2876 & 2970 & 2211 & $22 B$ & 259 & 282 & 282 & 368 & 338 & 336 & 355 & 389 & $38 B$ & 465 \\
\hline QUAD CITIES 2 & 1276 & 1417 & 1417 & 1581 & 1898 & 1898 & 1853 & 1994 & 1994 & 2128 & 2268 & 241 & 288 & 268 & 292 & 318 & 318 & 343 & 358 & 358 & 392 & 416 \\
\hline RANCHO SECO-1 & 265 & 318 & 318 & 373 & 432 & 432 & 498 & 552 & 552 & 883 & 657 & 123 & 147 & 147 & 173 & 290 & 280 & 231 & 258 & 256 & 279 & 304 \\
\hline ROBINSON 2 & 222 & 268 & 362 & 354 & 354 & 394 & 446 & 440 & 491 & 531 & 531 & 98 & 115 & 130 & 151 & $15 i$ & 169 & 189 & 189 & 269 & 227 & 227 \\
\hline RYR BEND 1 & 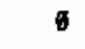 & $\theta$ & 130 & 317 & 317 & 488 & 682 & 682 & 858 & 1031 & 1031 & 6 & 6 & 24 & 59 & 59 & 96 & 128 & 128 & 258 & 191 & 191 \\
\hline SALE UNIT 2 & 115 & 163 & $\$ 63$ & 243 & 317 & 317 & 399 & 471 & 471 & 535 & $B 66$ & 53 & 75 & 75 & 112 & 148 & 148 & 183 & 216 & 218 & 246 & 278 \\
\hline SALE 1 & 270 & 341 & 462 & 402 & 474 & 538 & 536 & 621 & 892 & 692 & 778 & 124 & 157 & 185 & 185 & 218 & 248 & 246 & 285 & 318 & 318 & 357 \\
\hline SAN ONBFRE 1 & 94 & 141 & 141 & 188 & 231 & 231 & 231 & 277 & 277 & 320 & 329 & 35 & 52 & 52 & 89 & 85 & 85 & 85 & 102 & 102 & 128 & 118 \\
\hline SAN ONOFRE 2 & 68 & 148 & $2: 7$ & 217 & 292 & 282 & 378 & 378 & 452 & 452 & 530 & 28 & 81 & 98 & 90 & 122 & 122 & 158 & 158 & 190 & 190 & 223 \\
\hline SAN ONOFRE 3 & 86 & 147 & 347 & 223 & 223 & 298 & 298 & 377 & 377 & 452 & 530 & 28 & 60 & 68 & 92 & 92 & 124 & 124 & 158 & 158 & 190 & 223 \\
\hline SEABRDOK 1 & 6 & 0 & 6 & 6 & 76 & 120 & 173 & 173 & 222 & 287 & 325 & 8 & $\theta$ & 0 & a & 35 & 55 & 80 & 80 & 102 & 123 & 150 \\
\hline SEAEROOK 2 & 9 & $\theta$ & 0 & $\theta$ & 0 & 6 & 0 & 8 & - & 6 & a & g & 6 & $\theta$ & 0 & $\theta$ & 0 & 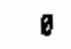 & $\theta$ & 0 & 8 & \\
\hline
\end{tabular}


TABLE A.4. Upper Reference Case, 1985 Inventory and Projected Inventory to 2020 (contd)

\begin{tabular}{|c|c|c|c|c|c|c|c|c|c|c|c|c|c|c|c|c|c|c|c|c|c|c|}
\hline \multirow[b]{2}{*}{ REACTOR } & \multirow{2}{*}{$\frac{\text { Inv }}{1985}$} & \multicolumn{10}{|c|}{ Assenblies } & $\frac{\operatorname{In} y}{\ln }$ & \multicolumn{10}{|c|}{ 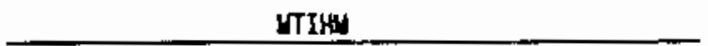 } \\
\hline & & $\underline{1986}$ & $\underline{1987}$ & $\underline{1988}$ & 1989 & $\underline{1990}$ & $\underline{1991}$ & $\underline{1992}$ & $\underline{1993}$ & 1994 & 1995 & $\underline{1985}$ & 1986 & 1987 & 1988 & 1989 & 1998 & 1991 & $\underline{1992}$ & 1993 & $\underline{1994}$ & 1995 \\
\hline SEQUOYAH 1 & 212 & 212 & $\overline{212}$ & $\overline{280}$ & $\overrightarrow{289}$ & $\overline{348}$ & $\overline{348}$ & 410 & 496 & 488 & $\overline{557}$ & $: \quad 97$ & 97 & 97 & 129 & $\overline{129}$ & $\overline{168}$ & $\overline{160}$ & $\overline{192}$ & $\overline{223}$ & $\overline{223}$ & 56 \\
\hline SEQCOYAH 2 & 130 & 200 & 289 & 278 & 278 & 346 & 346 & 417 & 417 & 404 & 554 & 62 & 96 & 96 & 128 & 128 & 159 & 158 & 192 & 192 & 223 & 55 \\
\hline SHOREAN & • & - & $\bullet$ & $\bullet$ & - & $\theta$ & 145 & 145 & 287 & 386 & 388 & $\theta$ & 6 & - & $\theta$ & 0 & - & 27 & 27 & 53 & 71 & 71 \\
\hline SOUTH TEXAS, U & 6 & 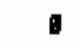 & 6 & 1. & 70 & 112 & 152 & 195 & 236 & 274 & 323 & 8 & $\theta$ & 6 & 1 & 38 & 61 & 82 & 186 & 27 & 148 & 75 \\
\hline SOUTH TEXAS, U & 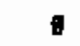 & $\theta$ & a & 6 & ! & 6 & 48 & 94 & 134 & 171 & 226 & 6 & 0 & 0 & 6 & 0 & 6 & 25 & 51 & 73 & 93 & 122 \\
\hline ST LUCIE 1 & 444 & 444 & 499 & 564 & 564 & 824 & 697 & 897 & 760 & 823 & 823 & 169 & 189 & 198 & 214 & 214 & 238 & 284 & 284 & 288 & 312 & 312 \\
\hline ST LUXIE 2 & 86 & 158 & 200 & 200 & 272 & 333 & 333 & 397 & 457 & 467 & 521 & 31 & 59 & 79 & 79 & 103 & 127 & 127 & 51 & 175 & 75 & 0 \\
\hline MER I & 112 & 112 & 164 & 223 & 223 & 280 & 345 & 346 & 462 & 450 & 459 & 51 & $\$ 1$ & 75 & 102 & 102 & 129 & 169 & 59 & 85 & & 12 \\
\hline SURRY I & 474 & 521 & 621 & 568 & 811 & 665 & 855 & 762 & 745 & 745 & 792 & 215 & 237 & 237 & 257 & 278 & 298 & 298 & 320 & 139 & 339 & 61 \\
\hline SURRY 2 & 382 & 414 & 414 & 451 & 499 & 498 & 569 & 697 & 597 & 841 & 88 & 164 & 188 & 188 & 265 & 227 & 227 & 258 & 272 & 272 & 292 & 14 \\
\hline SUSQUEHAR & 192 & 484 & 647 & 647 & 851 & 1038 & 1938 & 1297 & 1425 & 1425 & 1823 & 36 & 85 & 119 & 119 & 155 & 188 & $1 B 8$ & 222 & 255 & 255 & 89 \\
\hline SUSQUE & 0 & $\theta$ & 246 & 429 & $42 \theta$ & 615 & 830 & 839 & 1018 & 1265 & 1203 & $\theta$ & $\theta$ & 48 & 79 & 79 & 113 & 158 & 150 & 182 & 215 & 215 \\
\hline UILE & 208 & 298 & 268 & 393 & 333 & 394 & 489 & 496 & 521 & 582 & 582 & 97 & 87 & 123 & 154 & 154 & 183 & 213 & 213 & 242 & & \\
\hline TROJAN & 319 & 351 & 40: & 455 & 497 & 537 & 503 & 625 & 685 & 705 & 747 & 147 & 161 & 184 & 299 & 228 & 247 & 288 & 87 & 396 & 24 & 44 \\
\hline TURKEY $P$ & 424 & 424 & 487 & 588 & 588 & 552 & 598 & 598 & 630 & 878 & 78 & 192 & 192 & 12 & 231 & 231 & 251 & 272 & 72 & 91 & 89 & \\
\hline TLRKEY PT 4 & 378 & 441 & 480 & 480 & 524 & 585 & 565 & 697 & 847 & 647 & 880 & 171 & 260 & 218 & 218 & 238 & 257 & 257 & 276 & 295 & 95 & 14 \\
\hline YOGTLE 1 & 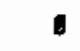 & $\bullet$ & 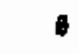 & ! & 111 & 178 & 178 & 250 & 314 & 314 & 392 & o & 6 & 0 & v & 51 & 81 & B1 & 115 & 145 & 45 & 3. \\
\hline YDGTLE 2 & 6 & - & - & 6 & $\theta$ & 64 & 64 & 139 & 203 & 293 & 289 & 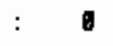 & 0 & 6 & 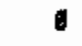 & 6 & 39 & 30 & 84 & 94 & 94 & 25 \\
\hline VT YAN & 1322 & 1322 & 1412 & 1522 & 1522 & 1830 & 1752 & 1752 & 1858 & 1906 & 1985 & 248 & 248 & 283 & 283 & 283 & 303 & 325 & 25 & 344 & 64 & 84 \\
\hline WASH MUCLAR 2 & - & 122 & 273 & 418 & 544 & 879 & 832 & 977 & 1111 & 1245 & 1387 & $\theta$ & 22 & 59 & 77 & 100 & 124 & 151 & 77 & & & \\
\hline ATERORO 3 & 6 & 68 & $6 B$ & 149 & 149 & 234 & 331 & 331 & 415 & 415 & 498 & $\theta$ & 29 & 29 & 81 & 61 & 87 & 137 & 137 & 172 & 172 & 18 \\
\hline WATTS BAR 1 & 0 & $\theta$ & 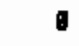 & 6 & - & $\theta$ & 46 & 116 & 115 & 176 & 170 & 6 & - & $\theta$ & 6 & 1 & 6 & 21 & 53 & 53 & 8 & $7 \varepsilon$ \\
\hline WATTS BAR 2 & : & - & 6 & 0 & 5 & - & 48 & 49 & 104 & 164 & 170 & $\theta$ & $\sigma$ & - & & $\theta$ & 6 & 22 & 22 & 48 & 18 & 78 \\
\hline WNP-1 & 6 & - & 6 & - & 0 & 6 & 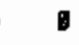 & & 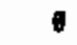 & 8 & & 6 & $\theta$ & B & 1 & 6 & 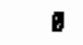 & & 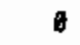 & & & \\
\hline WNP-3 & $\theta$ & 6 & 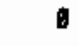 & 6 & - & $\theta$ & 0 & 6 & 0 & 6 & 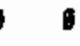 & 6 & & 6 & 0 & - & 0 & a & & & & \\
\hline NOLF CF & $\theta$ & 47 & 66 & 127 & 188 & 269 & 256 & 297 & 337 & 377 & 419 & $\theta$ & 22 & 40 & 59 & 78 & 97 & 118 & 137 & 156 & 174 & 94 \\
\hline YANKEE-RONE ? & 341 & 341 & 377 & 411 & 411 & 442 & 481 & 481 & 511 & 511 & 540 & 83 & 83 & 81 & 99 & 99 & 166 & 115 & 115 & 122 & 122 & 130 \\
\hline ZION 1 & 516 & 568 & 825 & 625 & 886 & 747 & 747 & 810 & 879 & 870 & 933 & 233 & 259 & 286 & 285 & 313 & 341 & 341 & 370 & 397 & 397 & 126 \\
\hline ZION 2 & 503 & 503 & 584 & 828 & 626 & 890 & 759 & 758 & 819 & 879 & & 229 & 229 & 257 & 285 & 286 & 314 & 348 & 346 & 373 & 491 & 101 \\
\hline BRUNSWIO & 160 & 180 & 180 & 160 & 180 & 168 & 168 & 160 & 180 & 180 & 180 & 71 & 71 & 71 & 71 & 71 & 71 & 71 & 71 & 71 & 1 & 71 \\
\hline BRUNSWICK 2 PAR & 144 & 144 & 144 & 144 & 144 & 144 & 144 & 144 & 144 & 144 & 144 & 88 & 88 & 68 & 68 & 88 & 68 & 88 & 88 & 86 & 86 & 66 \\
\hline HARRIS 1 GMR POOL & - & - & & - & 6 & - & 6 & $\theta$ & 6 & ] & - & 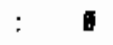 & 6 & 1 & $\theta$ & 1 & 6 & 6 & 6 & 0 & 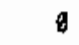 & \\
\hline WORRIS-BWR & 1627 & 1527 & 1627 & 1527 & 1527 & 1527 & 1527 & 1527 & 1527 & 1527 & 1527 & 290 & 296 & 298 & 298 & 290 & 299 & 290 & 290 & 299 & 298 & 290 \\
\hline MORRIS-PAR & 350 & 368 & 354 & 350 & 350 & 350 & 350 & 364 & 350 & 360 & 356 & 132 & 132 & 132 & 132 & 132 & 132 & 132 & 132 & 132 & 132 & 132 \\
\hline WEST VALEY-B & 86 & 85 & 85 & 65 & 85 & $B 5$ & 86 & 85 & as & 85 & & 11 & 11 & 11 & 11 & 11 & 11 & 11 & 11 & 11 & 1 & 11 \\
\hline WEST VNШEY P & 120 & 129 & 120 & 126 & 120 & 120 & 120 & 120 & 120 & 120 & 120 & 47 & 47 & 47 & 47 & 47 & 47 & 47 & 47 & 47 & 47 & 47 \\
\hline BUR GENERIC & 0 & $\theta$ & 1 & $\theta$ & 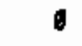 & 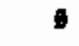 & 0 & 6 & D & 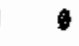 & 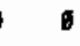 & $\theta$ & D & $\theta$ & 0 & 4 & 0 & 0 & 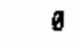 & $\theta$ & 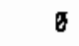 & \\
\hline PAR GENERIC & 6 & 9 & 1 & 6 & 6 & 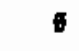 & $\theta$ & v & - & 6 & 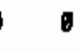 & 8 & 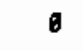 & $\sigma$ & 6 & 0 & 0 & 6 & 8 & $\sigma$ & $\theta$ & 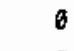 \\
\hline TOTNL GENERIC & $\theta$ & 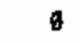 & 8 & 4 & $\theta$ & $\theta$ & 1 & $\theta$ & 1 & 6 & & 8 & 0 & 6 & $\theta$ & 0 & (1) & 6 & 0 & 6 & 6 & \\
\hline
\end{tabular}


TABLE A.4. Upper Reference Case, 1985 Inventory and Projected Inventory to 2020 (contd)

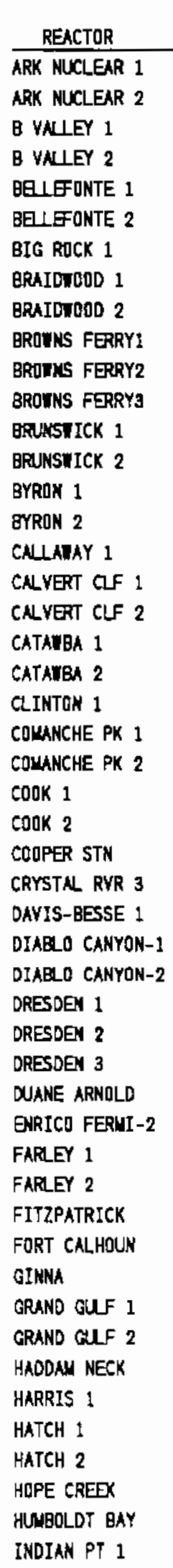

Assenblies

VTIH

\begin{tabular}{|c|c|c|c|c|c|c|c|c|c|c|c|c|c|c|c|c|c|c|c|}
\hline & & & & & & & & & & & & & & & & & & & \\
\hline 33 & 733 & 778 & 778 & & 826 & & & & & & 146 & & & & & 461 & & & \\
\hline 326 & & 801 & & & & & & & & & & & & & & & & & \\
\hline 01 & & & & & & & & & & & & & & & & & & & \\
\hline 35 & 369 & 380 & 53 & 415 & 15 & & & & 58 & & & & & 91 & & & & & 257 \\
\hline a & 1 & 0 & $\theta$ & ] & $\theta$ & 51 & 5 & 118 & 184 & & 0 & 1 & & 1 & & 23 & 3 & 4 & 84 \\
\hline 6 & g & 9 & 38 & 38 & & & & & 299 & 6 & 0 & 0 & 17 & 17 & 1 & 7 & 7 & & 136 \\
\hline 343 & 359 & 375 & 390 & 408 & 424 & 424 & & & 72 & 45 & 47 & 49 & 51 & 53 & 55 & 00 & iB & 10 & 6 \\
\hline 399 & & 550 & 550 & 623 & 695 & 396 & & & 815 & & $a$ & & 33 & 284 & & 94 & 18 & & 34 \\
\hline 293 & 90 & 448 & 448 & 528 & 599 & 598 & 88 & & 35 & & 81 & & 96 & 22 & 3 & 53 & & & 1. \\
\hline 189 & 2073 & 2973 & 2252 & 2252 & 2433 & 2594 & 2594 & 2783 & 2783 & 48 & 381 & 381 & 14 & 414 & 46 & 76 & 76 & 10 & 510 \\
\hline & 23 & & & & & 913 & & & 103 & & 39 & & 71 & 471 & 84 & 34 & & 58 & 68 \\
\hline 93 & & & 68 & 2388 & & 48 & & & 114 & & 367 & & 33 & 433 & 38 & 186 & 33 & 03 & 532 \\
\hline & & & 76 & 2234 & & & & & & & & & 17 & 18 & & 41 & & & 498 \\
\hline 91 & 1701 & 1851 & 1998 & 1998 & 2143 & 2274 & 2274 & 28 & 557 & & 16 & & 71 & 371 & & 23 & & & 478 \\
\hline 504 & 571 & 571 & 637 & 799 & 769 & 769 & 845 & 845 & 994 & & 242 & 242 & 69 & 300 & 60 & 25 & 57 & 57 & 382 \\
\hline $4 \mathrm{~B} 6$ & 456 & 524 & 575 & 575 & 647 & 898 & 696 & 703 & 889 & & 93 & 222 & 43 & 243 & 14 & 194 & 94 & 23 & 342 \\
\hline 615 & 15 & 93 & 782 & 782 & B38 & 900 & 890 & 97 & 1041 & & 288 & 299 & 328 & 328 & 31 & 387 & 37 & 20 & 447 \\
\hline 96 & 18 & 1070 & 1076 & $114 \theta$ & 48 & 213 & 1213 & & 1289 & & 86 & 761 & 97 & 437 & & $46 !$ & & 0 & 498 \\
\hline 863 & 938 & 930 & 1088 & 1088 & 1084 & 1080 & 3184 & & 228 & & & & 83 & 383 & & 10 & & & 468 \\
\hline 495 & 548 & 598 & 598 & 653 & 704 & 749 & 807 & 867 & 852 & & 31 & 253 & 53 & 276 & & 117 & & & 388 \\
\hline 15 & 11 & 32 & 532 & 591 & 647 & 686 & 735 & 35 & 770 & & 203 & 225 & 25 & 250 & & 198 & & & 26 \\
\hline 14 & $14 \%$ & 1587 & 1587 & 1774 & 1934 & 1934 & 2198 & 2247 & 2247 & & 281 & 290 & 98 & 324 & 54 & 354 & & 11 & 41: \\
\hline 512 & & 26 & 669 & 724 & 770 & 818 & & & 957 & & & & 35 & 197 & & 45 & & 3 & 401 \\
\hline 352 & 13 & 484 & 597 & 581 & 812 & 653 & & & 793 & & & & & 236 & & & & & \\
\hline 197 & 1007 & 1071 & 1071 & 1139 & 1283 & 1203 & 1276 & 1276 & 1332 & & 458 & 47 & 479 & 516 & 10 & 46 & & & 590 \\
\hline 71 & & 11 & 10 & 1085 & 1685 & 47 & 1147 & & 1282 & 371 & 460 & 490 & 428 & 458 & 58 & 83 & 33 & 2 & 38 \\
\hline 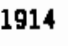 & 28 & & 2185 & & & 48 & & & 17 & & & & & 21 & & 52 & & & . \\
\hline 632 & 690 & 690 & 747 & 747 & 884 & 894 & 889 & 869 & 926 & & & 326 & & 347 & & & & & \\
\hline 558 & 865 & 885 & 852 & 704 & 704 & 746 & 261 & 891 & 843 & & 284 & 284 & 68 & 30 & 6 & 150 & & & 395 \\
\hline 597 & 80 & 89 & 675 & 875 & 757 & 757 & 855 & 355 & 927 & & 71 & 271 & 310 & 310 & 48 & 348 & 13 & 93 & 426 \\
\hline & & & & & & & & & & & & & & 297 & & 331 & & & 372 \\
\hline 83 & & 33 & 83 & & 683 & 683 & & & 683 & & 76 & & 70 & 70 & & 70 & & & \\
\hline 32 & 2558 & 2886 & 2886 & 2621 & 2946 & 2946 & 3688 & 3226 & 3220 & 36 & 451 & 472 & 72 & 495 & 18 & 518 & 40 & & 62 \\
\hline 76 & 2 & 24 & 2622 & 2622 & 47 & 2859 & & & 3989 & & & & 81 & 461 & 32 & 61 & 11 & & 41 \\
\hline & & & & & & & & & & & & & & & & & & & 6 \\
\hline 135 & & & 1671 & & & & & & $\$ 87$ & & & & 65 & 50 & & & & & \\
\hline 777 & 835 & 891 & 891 & 953 & 1607 & 1007 & 1872 & 1130 & 1130 & 358 & 384 & 410 & 416 & 439 & 64 & 464 & 14 & & 2. \\
\hline 657 & 657 & 715 & 789 & 769 & 827 & B75 & 75 & & 983 & & & 29 & 84 & 354 & 81 & 163 & 33 & & 153 \\
\hline 2649 & & 21 & & & & 2860 & & & & & & & & 452 & & & & & 024 \\
\hline & & & 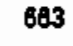 & 701 & 100 & 102 & 773 & & 803 & & & & & & & & & & \\
\hline & & & 737 & 764 & 789 & & 841 & & 890 & & 250 & 259 & 267 & 276 & & 92 & & & 1 \\
\hline 69 & 1769 & 1984 & 2193 & 2193 & 2464 & 2592 & 2592 & 2812 & 2996 & 316 & 318 & 354 & 391 & 391 & 428 & 481 & 81 & & 33 \\
\hline 6 & $\theta$ & 0 & 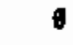 & 6 & $\theta$ & 289 & 515 & & 643 & 0 & 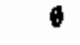 & 0 & 6 & 0 & 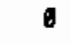 & 38 & 34 & 14 & 117 \\
\hline 997 & & 989 & 1031 & 163 & 1073 & 11 & 11 & & 1196 & & 391 & 497 & 425 & 425 & 442 & 458 & & 77 & 493 \\
\hline 312 & 000 & 300 & 398 & 443 & 770 & 410 & 514 & & 543 & 145 & 176 & & 185 & 296 & 200 & 221 & & & 0.4 \\
\hline & & & & & & & & & & & & 12 & 469 & 498 & & 522 & 53 & & 77 \\
\hline & & & 2076 & & & 2352 & & & 2833 & & 329 & 358 & 383 & 383 & 116 & 434 & 34 & & 187 \\
\hline 341 & 1341 & 1556 & 1718 & & 1934 & 2899 & 2090 & 2300 & 2444 & & 246 & 285 & 314 & 314 & 354 & 382 & 382 & 420 & 446 \\
\hline 390 & & & 390 & 390 & 390 & 390 & 390 & 396 & 390 & 2 & 29 & 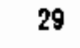 & 29 & 29 & 20 & 29 & 3 & & 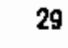 \\
\hline 160 & 180 & 160 & 180 & 160 & 160 & 180 & 160 & 180 & 180 & 31 & 31 & 31 & 31 & 31 & 31 & 31 & 31 & 31 & 5 \\
\hline
\end{tabular}


TABLE A.4. Upper Reference Case, 1985 Inventory and Projected Inventory to 2020 (contd)

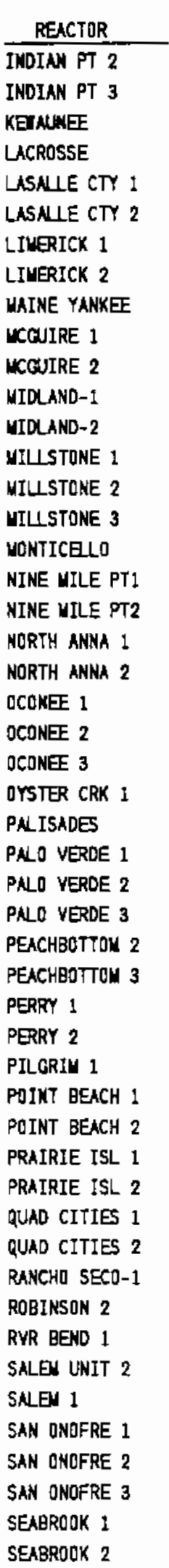

Assentilies

$\underline{1996} 1997199810992060209120022003$ 2004 2005

$002858 \quad 858 \quad 910 \quad \frac{988}{988} \frac{1016}{1616} \frac{1079}{1121}$ :

$\begin{array}{lllllllllllllllllllll}877 & 738 & 799 & 799 & 864 & 864 & 918 & 997 & 987 & 1046 & : & 399 & 337 & 365 & 385 & 395 & 395 & 419 & 451 & 451 & 475\end{array}$

$\begin{array}{lllllllllllllllllllll}710 & 740 & 770 & 799 & 831 & 868 & 888 & 920 & 951 & 977 & : & 274 & 285 & 296 & 397 & 320 & 331 & 346 & 353 & 385 & 375\end{array}$

$\begin{array}{lllllllllllllllllllll}381 & 381 & 389 & 386 & 401 & 491 & 417 & 417 & 437 & 437 & : & 46 & 46 & 49 & 49 & 53 & 53 & 56 & 58 & 59 & 58\end{array}$

$\begin{array}{llllllllllllllllllll}1461 & 1461 & 1838 & 1811 & 1811 & 1986 & 2142 & 2142 & 2326 & 2478 & 288 & 288 & 298 & 338 & 339 & 382 & 398 & 398 & 423 & 451\end{array}$

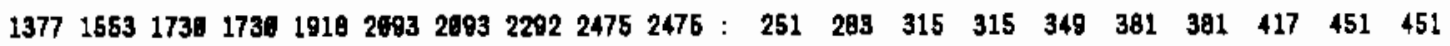

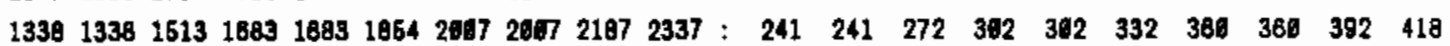

$\begin{array}{lllllllllllllllllllll}488 & 770 & 770 & 944 & 1177 & 1177 & 1358 & 1358 & 1594 & 1794: & 89 & 141 & 141 & 172 & 213 & 213 & 245 & 245 & 287 & 397\end{array}$

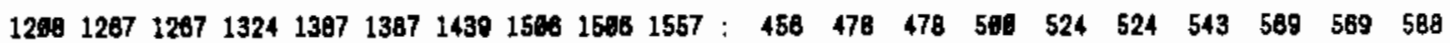

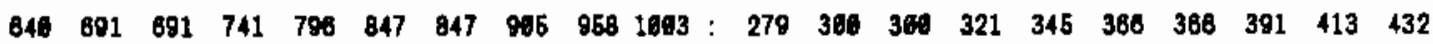
$\begin{array}{lllllllllllllllllllll}548 & 597 & 649 & 899 & 699 & 749 & 794 & 852 & 852 & 897 & : & 237 & 259 & 281 & 362 & 382 & 323 & 342 & 387 & 367 & 386\end{array}$

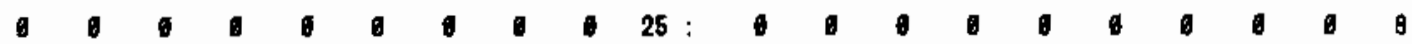

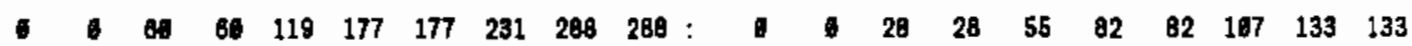

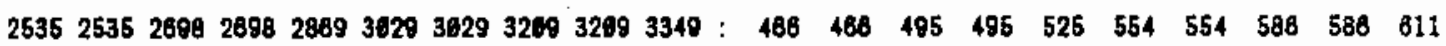
$\begin{array}{lllllllllllllllllllll}922 & 981 & 1039 & 1039 & 1101 & 1159 & 1150 & 1225 & 1285 & 1285 & : & 367 & 391 & 415 & 415 & 440 & 464 & 464 & 490 & 515 & 515\end{array}$ $\begin{array}{lllllllllllllllllllll}526 & 520 & 588 & 645 & 645 & 716 & 79 & 779 & 846 & 904 & : & 240 & 246 & 271 & 298 & 298 & 339 & 359 & 359 & 390 & 417\end{array}$

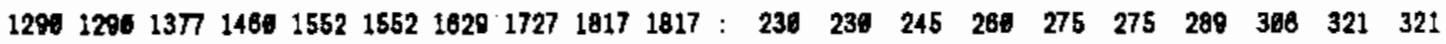

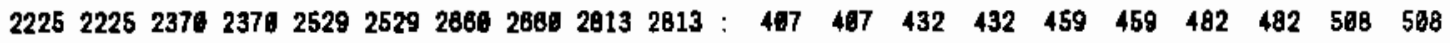
$\begin{array}{lllllllllllllllllllll}811 & 811 & 1029 & 1029 & 1251 & 1251 & 1416 & 1416 & 1837 & 1637 & : & 148 & 148 & 178 & 178 & 268 & 268 & 236 & 236 & 274 & 274\end{array}$ $\begin{array}{lllllllllllllllllllll}679 & 731 & 731 & 761 & 936 & 836 & 881 & 939 & 939 & 984 & : & 313 & 337 & 337 & 369 & 385 & 385 & 466 & 433 & 433 & 454\end{array}$ $\begin{array}{lllllllllllllllllllll}697 & 697 & 659 & 799 & 799 & 780 & 895 & 895 & 858 & 993 & : & 280 & 280 & 394 & 327 & 327 & 351 & 371 & 371 & 396 & 417\end{array}$

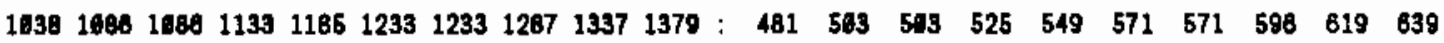
$\begin{array}{lllllllllllllllllllll}812 & 800 & 860 & 907 & 958 & 1606 & 1018 & 1061 & 1103 & 1145 & 376 & 399 & 399 & 420 & 444 & 486 & 468 & 492 & 511 & 531\end{array}$ $\begin{array}{lllllllllllllllllllll}747 & 795 & 844 & 891 & 891 & 938 & 998 & 988 & 1090 & 1072 & : & 347 & 389 & 392 & 413 & 413 & 435 & 455 & 455 & 478 & 497\end{array}$ $\begin{array}{lllllllllllllllllllll}2288 & 2328 & 2328 & 2458 & 2456 & 2596 & 2716 & 2718 & 2863 & 2982 & : & 404 & 425 & 425 & 447 & 447 & 472 & 493 & 493 & 519 & 549\end{array}$ $\begin{array}{lllllllllllllllllllll}892 & 882 & 937 & 999 & 996 & 1044 & 1644 & 1165 & 1181 & 1161 & : & 350 & 350 & 372 & 392 & 392 & 413 & 413 & 437 & 459 & 469\end{array}$ \begin{tabular}{lllllllllll|llllllllll}
613 & 864 & 716 & 768 & 821 & 872 & 917 & 975 & 1628 & 1973 & 282 & 284 & 397 & 329 & 353 & 375 & 394 & 428 & 443 & 462
\end{tabular} $\begin{array}{llllllllllllllllllll}828 & 692 & 742 & 786 & 842 & 893 & 942 & 998 & 1933 & 1077 & 284 & 293 & 315 & 333 & 358 & 391 & 462 & 423 & 442 & 461\end{array}$ $\begin{array}{lllllllllllllllllllll}497 & 594 & 556 & 695 & 654 & 769 & 748 & 890 & 852 & 897 & 185 & 215 & 237 & 254 & 286 & 304 & 321 & 344 & 366 & 382\end{array}$

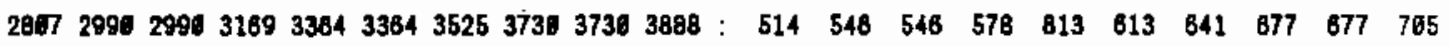

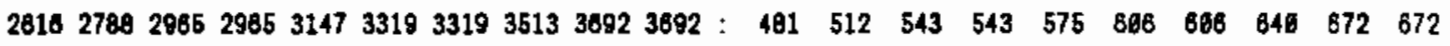

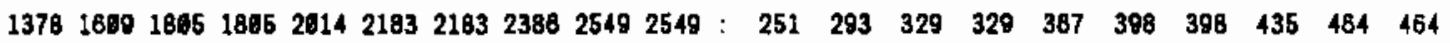
$\begin{array}{lllllllllllllllllllll}432 & 670 & 878 & 871 & 1131 & 1131 & 1331 & 1884 & 1664 & 1791: & 79 & 123 & 123 & 159 & 268 & 208 & 243 & 363 & 363 & 326\end{array}$

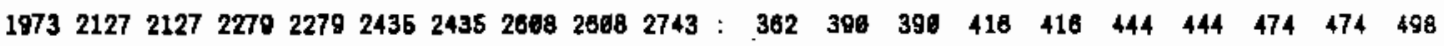
$\begin{array}{lllllllllllllllllllll}721 & 746 & 772 & 797 & 825 & 850 & 873 & 992 & 928 & 951 & : & 279 & 288 & 297 & 396 & 318 & 324 & 333 & 343 & 352 & 360\end{array}$ $\begin{array}{lllllllllllllllllllll}702 & 728 & 753 & 778 & 805 & 898 & 852 & 880 & 907 & 929 & 289 & 278 & 297 & 298 & 305 & 314 & 322 & 332 & 342 & 350\end{array}$ $\begin{array}{lllllllllllllllllllll}685 & 717 & 749 & 781 & 815 & 816 & 843 & 879 & 912 & 948 & 257 & 288 & 279 & 291 & 363 & 393 & 313 & 325 & 337 & 347\end{array}$ $\begin{array}{lllllllllllllllllllll}685 & 717 & 749 & 789 & 814 & 846 & 974 & 911 & 944 & 972 & 257 & 268 & 279 & 298 & 382 & 314 & 324 & 337 & 348 & 358\end{array}$ $\begin{array}{lllllllllllllllllllll}2329 & 2329 & 2458 & 2584 & 2584 & 2711 & 2824 & 2824 & 2958 & 3978 & 428 & 428 & 449 & 471 & 471 & 493 & 513 & 513 & 537 & 558\end{array}$ $\begin{array}{lllllllllllllllllllll}2268 & 2397 & 2525 & 2525 & 2881 & 2789 & 2789 & 2934 & 3687 & 3667 & 416 & 439 & 461 & 461 & 485 & 598 & 568 & 534 & 557 & 557\end{array}$ $\begin{array}{lllllllllllllllllllll}657 & 703 & 752 & 752 & 804 & 849 & 849 & 964 & 954 & 954 & 304 & 325 & 348 & 348 & 372 & 393 & 393 & 418 & 441 & 441\end{array}$ $\begin{array}{lllllllllllllllllllll}587 & 615 & 815 & 852 & 693 & 693 & 738 & 780 & 820 & 820 & : & 243 & 282 & 262 & 278 & 298 & 290 & 314 & 333 & 350 & 350\end{array}$

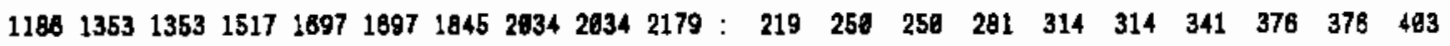
$\begin{array}{llllllllllllllllllllll}606 & 883 & 755 & 755 & 891 & 898 & 898 & 975 & 1845 & 1045 & 278 & 314 & 347 & 347 & 382 & 412 & 412 & 448 & 480 & 480\end{array}$ $\begin{array}{lllllllllll:llllllllll}838 & 838 & 910 & 980 & 980 & 1847 & 1108 & 1108 & 1176 & 1235 & : & 385 & 385 & 418 & 450 & 456 & 481 & 508 & 588 & 540 & 567\end{array}$ $\begin{array}{lllllllllllllllllllll}359 & 359 & 401 & 461 & 445 & 445 & 482 & 482 & 528 & 528 & 133 & 133 & 148 & 148 & 165 & 185 & 178 & 178 & 195 & 195\end{array}$ $\begin{array}{lllllllllllllllllllll}598 & 598 & 682 & 682 & 738 & 738 & 891 & 801 & 875 & 875 & 251 & 251 & 289 & 280 & 312 & 312 & 339 & 339 & 371 & 371\end{array}$ $\begin{array}{lllllllllllllllllllll}530 & 601 & 681 & 865 & 685 & 738 & 736 & 817 & 817 & 886 & : & 223 & 253 & 253 & 281 & 281 & 311 & 311 & 346 & 346 & 373\end{array}$ $\begin{array}{lllllllllllllllllllll}307 & 367 & 419 & 458 & 512 & 583 & 691 & 691 & 651 & 686 & 189 & 189 & 193 & 211 & 236 & 286 & 277 & 277 & 380 & 317\end{array}$

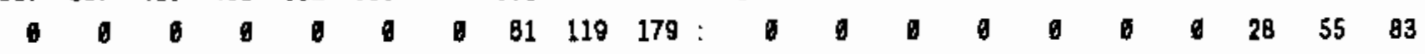


TABLE A.4. Upper Reference Case, 1985 Inventory and Projected Inventory to 2020 (contd)

\begin{tabular}{|c|c|c|c|c|c|c|c|c|c|c|c|c|c|c|c|c|c|c|c|c|}
\hline \multirow[b]{2}{*}{ REACTOR } & \multicolumn{10}{|c|}{ Assenblies } & \multicolumn{10}{|c|}{ ᄂTIHM } \\
\hline & 96 & 1997 & $\underline{1998}$ & 1890 & 2690 & 2001 & $\underline{2602}$ & 2003 & 2604 & $\underline{2095}$ & 19961 & $\underline{1997}$. & $\underline{1990}$ & 1999 & $\underline{2998}$ & 2091 & 2002 & 2063 & 2004 & $\underline{2005}$ \\
\hline DUOYAH 1 & 57 & 621 & 688 & 688 & 754 & 754 & B11 & 894 & 884 & 946 & 258 & 285 & 315 & 315 & 346 & 346 & 372 & 498 & 486 & 431 \\
\hline EQUDYAH 2 & 54 & 618 & 818 & 881 & 750 & $75:$ & 896 & 896 & 973 & 929 & 255 & 285 & 285 & 314 & 345 & 345 & 371 & 371 & 22 & 128 \\
\hline HOREDX시 & 68 & 678 & 678 & 793 & 929 & 929 & 1659 & 1174 & 1174 & 1382 & 93 & 124 & 124 & 145 & 170 & 170 & 193 & 214 & 14 & 237 \\
\hline UTH TEXAS, UNIT I & 358 & 416 & 457 & 492 & 542 & 587 & 826 & 685 & 708 & 736 & 194 & 225 & 247 & 268 & 293 & 318 & 339 & 361 & 378 & 398 \\
\hline DUTH TEXAS, UNIT 2 & 259 & 318 & 380 & 392 & 443 & 491 & 521 & 584 & 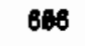 & 834 & 148 & 172 & 195 & 212 & 246 & 286 & 282 & 385 & 28 & 343 \\
\hline T LUCIE 1 & 79 & 948 & 948 & 999 & 1665 & 1085 & 1110 & 1188 & 1388 & 1241 & 334 & 357 & 357 & 380 & 485 & 165 & 425 & 452 & 52 & 672 \\
\hline LUCIE 2 & 674 & 574 & 832 & 689 & 689 & 747 & 798 & 798 & 858 & 988 & 226 & 220 & 243 & 285 & 285 & 288 & 67 & 367 & 31 & 350 \\
\hline MER 1 & 19 & 583 & 563 & 616 & 875 & 675 & 723 & 785 & 785 & 832 & 235 & 268 & 266 & 284 & 312 & 12 & 34 & 363 & 33 & 185 \\
\hline JRRY 1 & 1 & 831 & 874 & 915 & 915 & 968 & 993 & 993 & 1038 & 1672 & 379 & 370 & 398 & 418 & 418 & 438 & 453 & 453 & 73 & 498 \\
\hline JRRY 2 & 38 & 731 & 774 & 774 & 818 & 868 & 886 & 998 & 952 & 952 & 314 & 334 & 353 & 353 & 373 & 393 & 393 & 415 & 35 & \\
\hline ISQUEYAAY & 1791 & 1791 & 1973 & 2148 & 2148 & 2325 & 2483 & 2483 & 2878 & $2 B 26$ & 318 & 318 & 349 & 380 & 386 & 410 & 437 & 437 & 74 & 197 \\
\hline SQUEHANNA 2 & 1389 & 1549 & 1549 & 1724 & 1018 & 1918 & 2975 & 2276 & 2276 & 2432 & 243 & 274 & 274 & 305 & 338 & 338 & 65 & 480 & 60 & $\$ 27$ \\
\hline UILE & 835 & 835 & 893 & 749 & 749 & 897 & 858 & 858 & 918 & 969 & 294 & 294 & 321 & 347 & 347 & 374 & 398 & 390 & 426 & 449 \\
\hline $20 J N x$ & 32 & 821 & 880 & 898 & 939 & 977 & 1611 & 1055 & 1095 & 1129 & 369 & 378 & 396 & 413 & 432 & 450 & 465 & 486 & 64 & 528 \\
\hline TURKEY $P$ & 13 & 751 & 751 & 789 & 830 & 830 & 884 & 998 & 998 & 941 & 325 & 342 & 342 & 360 & 379 & 379 & 94 & 15 & 15 & 30 \\
\hline TURKEY PT 4 & 726 & 728 & 785 & 893 & 803 & 841 & 841 & 885 & 825 & 925 & & 91 & 349 & 366 & 388 & 384 & 184 & & 22 & 422 \\
\hline DGTE 1 & 56 & 450 & 618 & 575 & 576 & 642 & 765 & 705 & 772 & B30 & 268 & 268 & 239 & 285 & $2 B 6$ & 296 & 325 & 325 & 356 & 383 \\
\hline VOGTLE 2 & 39 & 339 & 497 & 483 & 483 & 530 & 595 & 595 & $B 82$ & 720 & 158 & 168 & 188 & 214 & 214 & 245 & 275 & 275 & 365 & 332 \\
\hline YANKEE 1 & 80 & 2182 & 2182 & 2261 & 2371 & 2371 & 2461 & 2576 & 2578 & 2884 & 381 & 400 & 406 & 418 & 438 & 439 & 55 & 476 & 176 & 492 \\
\hline ASH MU & 1567 & & 1768 & 1891 & 2627 & & & 2413 & & & & 93 & & 38 & 361 & 384 & 84 & & 3 & \\
\hline ATERFORD 3 & 570 & 570 & $B 44$ & 723 & 723 & 796 & 887 & 867 & 944 & 1015 & 238 & 236 & 287 & 300 & 390 & & 88 & 380 & 92 & \\
\hline TATTS BAR 1 & 228 & 369 & 389 & 359 & 359 & 431 & 485 & 485 & 546 & 540 & 104 & 143 & 143 & 168 & 188 & 199 & 224 & 224 & 249 & 249 \\
\hline WATTS BAR 2 & 225 & 225 & 287 & 287 & 381 & 429 & 429 & 489 & 489 & 544 & 104 & 164 & 132 & 132 & 167 & 198 & 198 & 228 & 28 & 251 \\
\hline WNP-1 & $\theta$ & 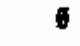 & $\theta$ & 6 & 6 & • & 0 & ต & 71 & 115 & 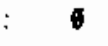 & . & 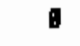 & 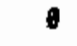 & 6 & 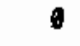 & ๑ & o & 32 & 52 \\
\hline P-3 & & & 230 & 281 & 357 & 426 & & 588 & & & & & 94 & 18 & 148 & & 01 & 48 & & \\
\hline JUF CREEK 1 & 456 & 493 & 531 & 589 & 810 & 848 & $B 82$ & 728 & 788 & 799 & 211 & 228 & 248 & 284 & 283 & 306 & 316 & 336 & 55 & 370 \\
\hline YANKEE-ROTE 1 & 573 & 573 & 649 & $B 49$ & 849 & 849 & 640 & 648 & $B 49$ & 849 & 137 & 137 & 154 & 154 & 154 & 154 & 154 & 154 & 154 & 154 \\
\hline ZION 1 & 88 & 986 & 1044 & 1191 & 1161 & 1158 & 1260 & 1209 & 1289 & 1320 & 456 & 450 & 477 & 503 & 593 & 529 & 552 & 552 & 880 & 603 \\
\hline $\operatorname{zION} 2$ & & 2000 & 998 & 1848 & 1197 & 1107 & 1158 & 1224 & 1224 & 1274 & 425 & 461 & 451 & 477 & 595 & 505 & 528 & 558 & 558 & 581 \\
\hline BRAASTICK 1 PNR & 160 & 168 & 180 & 160 & 180 & 186 & 168 & $18 B$ & 160 & 160 & 71 & 71 & 71 & 71 & 71 & & 71 & & & \\
\hline BRUNSWICK 2 PUR & 144 & 144 & 144 & 244 & 144 & 144 & 144 & 144 & 144 & 144 & 68 & 86 & B6 & 86 & 86 & 86 & 86 & 88 & 86 & 86 \\
\hline HARRIS I BIR POOL & $\theta$ & 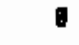 & 0 & $\theta$ & 6 & 6 & $\theta$ & $\theta$ & 0 & 6 & 6 & 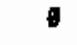 & 6 & $\theta$ & - & b & 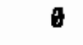 & 6 & $\theta$ & \\
\hline ORRIS-ERR & 1527 & 1527 & 1527 & 1527 & 1527 & 1527 & 1527 & 1527 & 1527 & 1627 & 298 & 290 & 296 & 290 & 290 & 290 & 298 & 296 & 296 & 290 \\
\hline NORRIS-PWR & 350 & 350 & 356 & 350 & 350 & 350 & 350 & 358 & 350 & 350 & 132 & 132 & 132 & 132 & 132 & 132 & 132 & 132 & 132 & 132 \\
\hline TEST VNLEY-B & 85 & 85 & 85 & 85 & 85 & 85 & 85 & 85 & 85 & $B 5$ & 11 & 11 & 11 & 11 & 11 & 11 & 11 & 11 & 11 & 11 \\
\hline WEST VALEY-P & 128 & 120 & 126 & 120 & 120 & 128 & 120 & 126 & 128 & 120 & 47 & 47 & 47 & 47 & 47 & 47 & 47 & 17 & 47 & 47 \\
\hline BWR GENERIC & 8 & 0 & 0 & 10 & 8 & b & 0 & 202 & 415 & 952 & b & 9 & 0 & - & $\theta$ & 0 & $\theta$ & 37 & 76 & 173 \\
\hline PWR GENERIC & 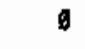 & 0 & 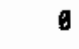 & 0 & $\theta$ & 6 & $\theta$ & 78 & 398 & 749 & 6 & 0 & $\theta$ & 8 & 0 & 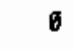 & ( & 35 & 183 & 344 \\
\hline TOTAL GEYERIC & 6 & & 6 & 0 & 6 & 0 & $\theta$ & 278 & 813 & 1701 & 1 & 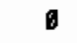 & - & 8 & 6 & 6 & 6 & 72 & 259 & 517 \\
\hline
\end{tabular}


TABLE A.4. Upper Reference Case, 1985 Inventory and Projected Inventory to 2020 (contd)

Assemblies

VTIHU

REACTOR

ARK WUCEAR 1 NRK WUCAR 2

Q VNUEY 1

B VNШEY 2

BELFONTE :

BELEONTE 2

gIG ROCK 1

BRAIDTOOO I

ARAIDTOOD 2

BRDTNS FERRY1

BROTNS FERTY2

BROINS FERRY3

BADUSTICK 1

gRULSIICK 2

BYRON 1

BYRON 2

CNLAIAY 1

CNVET CF 1

CNVERT CLF 2

CATATBA 1

CATATEA 2

CLIMTON 1

COMANHE PX 1

COUANCHE PK 2

COOK 1

cook 2

CQDPER STM

CRYSTNL RYR 3

DAVIS-BESSE 1

OIAQLO CAYOH-1

DIABLO CANON-2

DREDDE 1

ORESOEN 2

DRESDEY 3

DUANE ARNOLLD

EIRICO FERA:-2

FARLE 1

FAREE 2

FITZPATRICK

FORT CALHQUN

GINNA

GRWO GF 1

GRAND GUF 2

HAODAH NECK

HARRIS 1

HATCH 1

HATCH 2

HOPE CREEX

HUMGOLDT BAY

INDIAN PT 1

\begin{tabular}{|c|c|c|c|c|c|c|c|c|c|c|c|c|c|c|c|c|c|c|c|}
\hline 9 & $\pi 7$ & 90 & 10 & 110 & 111 & 112 & 2013 & 114 & 115 & 2000 & 2007 & 2968 & 2900 & 2010 & 2011 & $2012 ?$ & 2013 & 2814 & 2015 \\
\hline 951 & 11 & 101 & 48 & 40 & 95 & 42 & 42 & 81 & 68 & 441 & 484 & 464 & 486 & 488 & $5 \% 7$ & 529 & 529 & 547 & 629 \\
\hline 203 & 962 & $m$ & 100 & $18 \% 8$ & 108 & 123 & 180 & 89 & 225 & 377 & 491 & 421 & 421 & 445 & 445 & 488 & 492 & 92 & 11 \\
\hline 113 & 1113 & .82 & 223 & 1287 & 1287 & 345 & 1405 & 105 & 457 & 513 & 513 & 535 & 583 & 593 & 583 & 20 & 47 & 47 & 671 \\
\hline 568 & 813 & S50 & 860 & 760 & 755 & 755 & 204 & 847 & 847 & 257 & 283 & 394 & 390 & 323 & 348 & 48 & 71 & 390 & 396 \\
\hline 184 & 258 & 315 & 315 & 381 & 448 & 449 & 503 & 583 & 504 & 84 & 117 & 144 & 44 & 173 & 295 & 295 & 29 & 29 & 257 \\
\hline 290 & 380 & 427 & 427 & 488 & 488 & 547 & 853 & 603 & 671 & 136 & 188 & 195 & 195 & 223 & 223 & 50 & 275 & 275 & 306 \\
\hline 558 & 556 & 558 & 558 & 558 & 568 & 566 & 558 & 558 & 558 & 73 & 73 & 73 & 73 & 73 & 73 & 73 & 73 & 73 & 73 \\
\hline 874 & 930 & 938 & 994 & 1952 & 652 & 110 & 1172 & 1172 & 1223 & 376 & 397 & 397 & 428 & 445 & 445 & 472 & 498 & 496 & 517 \\
\hline 897 & 871 & a71 & 942 & 1690 & 1606 & 382 & 1118 & 1118 & 1178 & 341 & 368 & 368 & 390 & 423 & 423 & 449 & 473 & 473 & 495 \\
\hline 2994 & 2984 & 3139 & 3331 & 3331 & 3520 & 3520 & 3708 & 3864 & 4828 & 547 & 547 & 575 & 610 & 610 & 644 & 644 & 678 & 706 & 645 \\
\hline 3363 & 383 & 3459 & 556 & 3650 & 349 & 848 & 4028 & 4184 & 4948 & 684 & 684 & 633 & 887 & 867 & 702 & 782 & 738 & 764 & 983 \\
\hline 115 & 115 & 271 & 71 & 3472 & 382 & 882 & 350 & 3050 & 4088 & 589 & 589 & 507 & 97 & 834 & 668 & 88 & 792 & 792 & 731 \\
\hline 2622 & 2822 & 2946 & 589 & 3500 & 3580 & 3584 & 3600 & 3560 & 3560 & 28 & 526 & 550 & 853 & 653 & 853 & 53 & 853 & 653 & 653 \\
\hline 2557 & 2719 & 2845 & 2845 & 3968 & 3159 & 3159 & 3311 & 3437 & 3989 & 478 & 506 & 530 & 530 & 56: & 589 & 589 & 617 & 641 & 744 \\
\hline 977 & 977 & 1034 & 104 & 1104 & 174 & 243 & 43 & 396 & 1368 & 413 & 413 & 437 & 17 & 37 & 497 & 528 & 528 & 550 & 574 \\
\hline 89 & 872 & 914 & 914 & 72 & 933 & 1033 & 1099 & 1138 & 1138 & 342 & 389 & 387 & 387 & 411 & 437 & 437 & 461 & 481 & 481 \\
\hline 1041 & 1125 & 1185 & 185 & 269 & 342 & 1342 & 1422 & 1482 & 1482 & 447 & 462 & 597 & 557 & 543 & 574 & 574 & 868 & 633 & 633 \\
\hline 1376 & 970 & 1432 & 1432 & 513 & 513 & 1580 & 1588 & 1849 & 1865 & 520 & 520 & 544 & 544 & 74 & 574 & 682 & 802 & 825 & 767 \\
\hline 2228 & 1368 & 1388 & 1383 & 1383 & 484 & 1469 & 1536 & 1538 & 1598 & 486 & 498 & 496 & 524 & 524 & 553 & 553 & 582 & 582 & 685 \\
\hline 997 & 583 & 963 & 1017 & 1873 & 128 & 1179 & $\$ 179$ & 1223 & 1269 & 384 & 407 & 407 & 436 & 454 & 477 & 499 & 499 & 517 & 537 \\
\hline 826 & B75 & 918 & 918 & al & 298 & $\$ 1958$ & 158 & 1094 & 1135 & 349 & 376 & 387 & 87 & 97 & 428 & 447 & 447 & 483 & 480 \\
\hline 2427 & 2550 & 2558 & 2725 & 2883 & 2863 & 3015 & 3134 & 3134 & 3284 & 444 & 468 & 488 & 498 & 523 & 523 & 551 & 573 & 573 & 597 \\
\hline 1018 & 5053 & 1002 & 1150 & 1192 & 238 & 1288 & 324 & 1382 & 1406 & 425 & 440 & 458 & 479 & 496 & 518 & 535 & 49 & 565 & 582 \\
\hline 847 & 896 & 939 & 982 & 1925 & 975 & 25 & & 289 & 1249 & 15 & 385 & 79 & & & & 57 & 5 & 11 & 87 \\
\hline 1481 & 401 & 1458 & 1523 & 1523 & 1589 & 1689 & 1065 & 1769 & 1982 & 831 & 231 & 658 & 887 & 887 & 717 & 717 & 748 & 773 & 861 \\
\hline $12 \theta 2$ & 368 & 118 & 418 & 1495 & 105 & 1587 & 1039 & 1839 & t699 & 538 & 588 & 592 & 592 & 624 & 624 & 853 & 682 & 682 & 726 \\
\hline 2815 & 2966 & 2979 & 3.163 & 3102 & 240 & & & & 4940 & 8 & $\$ 35$ & 548 & 34 & & 17 & 3 & 8 & 12 & 42 \\
\hline 924 & 983 & 983 & 1043 & 1043 & 1162 & 1102 & 1182 & 1182 & 1210 & 427 & 458 & 458 & 484 & 484 & 511 & 511 & 539 & 539 & 561 \\
\hline 890 & 896 & 87 & 987 & 37 & 37 & 1687 & 1897 & 128 & 1176 & 429 & 420 & 439 & 483 & 463 & 488 & 510 & 510 & 529 & 548 \\
\hline 927 & 1921 & 1021 & 1100 & 1100 & 198 & 1198 & 1283 & 283 & 359 & 16 & 469 & 469 & & & & 10 & 9 & 9 & 24 \\
\hline 901 & 91 & 978 & 978 & 1606 & 1008 & 1155 & 1155 & 1225 & 1225 & 414 & 414 & 448 & 448 & 489 & 489 & 530 & 530 & 582 & 582 \\
\hline 183 & & 693 & & & & & & & 883 & 70 & 70 & 70 & & 0 & 6 & 19 & 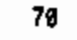 & 10 & 78 \\
\hline 2357 & 194 & 194 & 829 & 353 & 153 & 353 & 153 & 353 & 4353 & 15 & 698 & 608 & 10 & 751 & 751 & 751 & 51 & 751 & 751 \\
\hline$\infty$ & 36 & 43 & 343 & 480 & 11 & 135 & 35 & 335 & 4335 & 11 & 584 & 502 & 32 & 695 & 627 & 748 & 748 & 748 & 748 \\
\hline 2165 & 2105 & 187 & 2286 & 2286 & 388 & 485 & 2485 & 588 & 2854 & 12 & 382 & 398 & & 414 & 432 & 19 & 19 & 164 & 489 \\
\hline 2687 & 2839 & 2839 & 3988 & 3338 & 138 & 3578 & 578 & 3773 & 3773 & 72 & 518 & 518 & 33 & 869 & 869 & 652 & 852 & 688 & 588 \\
\hline 1193 & 1254 & 1254 & 314 & 1375 & 975 & 133 & 1493 & 493 & 1541 & 550 & 578 & 578 & & 834 & 634 & $88 \%$ & 688 & 688 & 710 \\
\hline 983 & 1046 & 1693 & 1993 & 1158 & 1214 & 1214 & 1274 & 1321 & 1321 & 453 & 482 & 564 & 34 & 533 & 559 & 559 & 587 & 609 & 689 \\
\hline 3995 & 3835 & 3157 & 3393 & 3963 & 3449 & 3591 & 3591 & 3711 & 3833 & 551 & 551 & 572 & 10 & 598 & 624 & 658 & 56 & 671 & 692 \\
\hline 842 & 842 & 971 & 907 & 907 & 43 & 977 & 1110 & 1110 & 1110 & 304 & 394 & 314 & & 327 & 346 & 353 & 160 & 406 & 400 \\
\hline 918 & 946 & 988 & 94 & 322 & 43 & 43 & 43 & 143 & 43 & 28 & 338 & 343 & & 81 & 01 & 61 & 81 & 81 & 401 \\
\hline 2998 & 3227 & 3497 & 87 & 3839 & 3859 & 859 & 4978 & 4258 & 258 & 13 & 573 & 695 & 5 & 848 & 885 & 685 & 723 & 755 & 755 \\
\hline 936 & 930 & 1105 & 1374 & 1374 & 1818 & 1885 & 1B85 & 2090 & 2329 & 188 & 168 & 199 & & 47 & 290 & 13 & 33 & 73 & 415 \\
\hline 1242 & 1288 & 1445 & 1445 & 1445 & 1445 & 1445 & 1445 & 1445 & 1445 & 511 & 530 & 595 & 595 & 595 & 595 & 595 & 595 & 595 & 595 \\
\hline 589 & 589 & 915 & $\$ 89$ & & 699 & & & 789 & 983 & 274 & 274 & 288 & & 387 & 325 & 343 & 343 & 358 & 373 \\
\hline 3278 & 278 & 3404 & 558 & 3558 & 768 & 358 & 3058 & 543 & 4543 & 697 & 697 & 631 & & 659 & 887 & 15 & 15 & 842 & 842 \\
\hline 2833 & 792 & 2918 & 2916 & 3978 & 3228 & 3228 & 3389 & 3565 & 3505 & 487 & 516 & 539 & 539 & 589 & 597 & S97 & 625 & 648 & 648 \\
\hline 2444 & 2843 & 2777 & 2777 & 2957 & 3149 & 3149 & 3329 & 3484 & 3484 & 448 & 483 & 507 & 507 & 540 & 575 & 575 & 687 & 635 & 635 \\
\hline 3 & & 390 & 396 & 38 & & 390 & 390 & 1 & 396 & 3 & 29 & 29 & & 29 & 29 & 29 & 9 & 29 & 29 \\
\hline 180 & 160 & 180 & 168 & 160 & 180 & 169 & 180 & 160 & 186 & 31. & 31 & 31 & 31 & 31 & 31 & 31 & 31 & 31 & 31 \\
\hline
\end{tabular}


TABLE A.4. Upper Reference Case, 1985 Inventory and Projected Inventory to 2020 (contd)

REACTOR

INOIAN PT 2

INDIAY PT 3

KENNEE

LACOISSE

LSNUE CTY 1

LSNUE CTY 2

LIUERICK 1

LIUERICX 2

WAIME YANKEE

ICEUTRE 1

WCOUIRE 2

MIOUND-1

MIOUND-2

HILSTONE 1

NIUSTONE 2

UIUSTONE 3

NOMTICELO

NIME UILE PT1

NIME MILE PT2

NORTH ANM 1

NORTH NIMA 2

OCONEE 1

OCONEE 2

DCDNEE 3

OYSTER CRA 1

PN.ISADES

PND VERDE I

PNDO VERDE 2

PALO VERDE 3

PEACHBOTTOU 2

PEACHBOTTOY 3

PERT 1

PEATI 2

PILCRIV 1

PDIMT BEACH 1

PगIMT BEACH 2

PQAIRIE ISL 1

PRAIRIE ISL 2

QUAD CITIES !

QUAD CITIES 2

RAKCHO SECO-1

ROBINSON 2

RVR BEND:

SALE UNIF 2

SNAE 1

SAN ONGFRE 1

SAN ONDFRE 2

SAN ONOFRE 3

SEABROOK 1

SEABROOK 2
Arsenblies

\begin{tabular}{|c|c|c|c|c|c|c|c|c|c|c|c|c|c|c|c|c|c|c|c|}
\hline \multicolumn{10}{|c|}{ Disedidies } & \multicolumn{10}{|c|}{ N1Fing } \\
\hline & & & $\theta 0$ & 010 & 2011 & & & & & & & & 968 & $2010 ?$ & 111 & 12 & 13 & & \\
\hline & & & 36 & & & & & & & & & & 558 & 596 & & & & & \\
\hline & & & & & & & & & & & & & 29 & 80 & 66 & & 16 & 16 & it \\
\hline & & & 198 & & & & & & & & & & 421 & 433 & & & 488 & 177 & 23 \\
\hline & & & 148 & & 46 & 546 & 16 & 546 & & & & 85 & 73 & 73 & 73 & & & & \\
\hline 8 & 69 & 18 & 318 & 68 & 88 & 188 & 71 & & & 461 & 488 & 513 & 513 & 547 & 581 & & 14 & 41 & 41 \\
\hline & & & 41 & 33 & & & & 592 & & & & & 54 & 89 & 589 & & 54 & 54 & 82 \\
\hline & & & 370 & 58 & & & & 959 & & & & & 77 & 10 & 542 & & & 99 & 99 \\
\hline & & & 86 & 44 & 344 & & & & & & & & 392 & 420 & 420 & & 59 & 91 & 27 \\
\hline & & 70 & 29 & 29 & 88 & & & & & & & & 154 & 654 & & & 59 & 9 & 5 \\
\hline 39 & & & 56 & 11 & & & & & & & & & 497 & 526 & 529 & & 84 & & \\
\hline 964 & & & 63 & & & 1214 & & 1258 & & & & & 452 & 478 & 498 & & 528 & 59 & 57 \\
\hline 58 & 84 & 38 & 39 & 64 & 82 & 221 & 247 & 274 & & 18 & & & 48 & 54 & 83 & & & & \\
\hline 48 & 10 & 14 & 44 & 91 & 44 & 0 & 84 & & 781 & & & & 296 & 227 & & & & & \\
\hline & & 60 & 389 & 36 & & & & & & & & & 888 & & & & & & [1] \\
\hline & & 12 & 171 & & 335 & & & & & & & & 591 & 617 & & & 14 & & \\
\hline 4 & & & 26 & & 137 & 37 & & 1243 & & & & & 471 & & & & & & \\
\hline & & & & & & & & & & & & & 382 & 382 & & & & & \\
\hline & & & 11 & 3280 & 3792 & & & 378 & & & & & 568 & 85 & & & & & 7 \\
\hline & & & & & & & & & & & & & 359 & & & & & & \\
\hline & & & & & & & & 1283 & & & & & 499 & 525 & & & & & \\
\hline 03 & & 103 & 893 & 1068 & 1113 & 13 & & 1295 & & & & & 483 & 489 & & & & & \\
\hline & & & & 574 & & & & & & & & & & & & & & & \\
\hline & & 90 & 39 & 92 & & & & & & & & & 574 & & & & & & \\
\hline & & 94 & 13 & 13 & 1283 & 111 & 65 & 537 & & & & & 582 & & & & & & \\
\hline & & & & & & & & & & & & & & & & & & & \\
\hline & & 287 & & 1 & & 897 & & & & & & & 523 & & & & & & \\
\hline 29 & & 228 & 02 & 1338 & & 1445 & & 1544 & & & & & 554 & & & & & & \\
\hline & & & 66 & & & & & & & & & & 543 & & & & & & \\
\hline & & 224 & 77 & 1122 & 170 & 218 & 263 & 302 & 43 & & & & 485 & 34 & & & & & \\
\hline 98 & & & & & & & & & & & & & & & & & & & \\
\hline & & & & & & & & & & & & & 770 & & & & & & \\
\hline 63 & $\omega$ & 300 & 15 & 3276 & & 161 & 598 & 8 & & & & & 587 & 97 & & & & & \\
\hline & & & & 678 & & & & & & & & & & & & & & & \\
\hline 2743 & 43 & 174 & 174 & 3043 & 943 & 201 & 781 & 781 & 781 & & & & 521 & 551 & & & & & \\
\hline 98 & & & & & & & & & & & & & & & & & & & \\
\hline 957 & & & & & & & & & & & & & 386 & & & & & & \\
\hline 976 & 011 & 136 & 171 & 107 & 849 & 173 & 1296 & 328 & 28 & & & & 93 & 38 & & & & & \\
\hline & & & & & & & & & & & & & & & & & & & \\
\hline & & 17 & 17 & 457 & 591 & 591 & 3 & 4435 & 135 & & & & 880 & 625 & & & & & \\
\hline 97 & & & 63 & & & & & & & & & & 630 & & & & & & \\
\hline 195 & 1959 & 100 & 1.110 & 0.00 & & & & & & & & & 513 & 536 & & & & & \\
\hline & & & 985 & 1097 & 1657 & 214 & 214 & 214 & 214 & & & & 411 & 9 & & & & & \\
\hline & & & & & & & & & & & & & 498 & 498 & & & & & \\
\hline & & & & & & 464 & & & & & & & 579 & & & & & & \\
\hline & 1399 & & & & & & & 1637 & & & & & 627 & 31 & & & & & \\
\hline 572 & & & & & & 765 & & & & & & & 283 & 283 & & & & & \\
\hline & & & & & & & & & & & & & 461 & 481 & & & & & \\
\hline & & 20 & 1019 & & & 1170 & & & & & & & 432 & 485 & & & & & \\
\hline & & & 823 & & 915 & 984 & 1007 & 1045 & 1845 & 342 & & & 380 & 496 & & 15 & & 32 & 482 \\
\hline 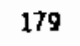 & 233 & 281 & 342 & 398 & 454 & 404 & 588 & 301 & 010 & 80 & 108 & tove & 158 & 100 & & & & & \\
\hline
\end{tabular}

A. 32 


\section{TABLE A.4. Upper Reference Case, 1985 Inventory and Projected Inventory to 2020 (contd)}

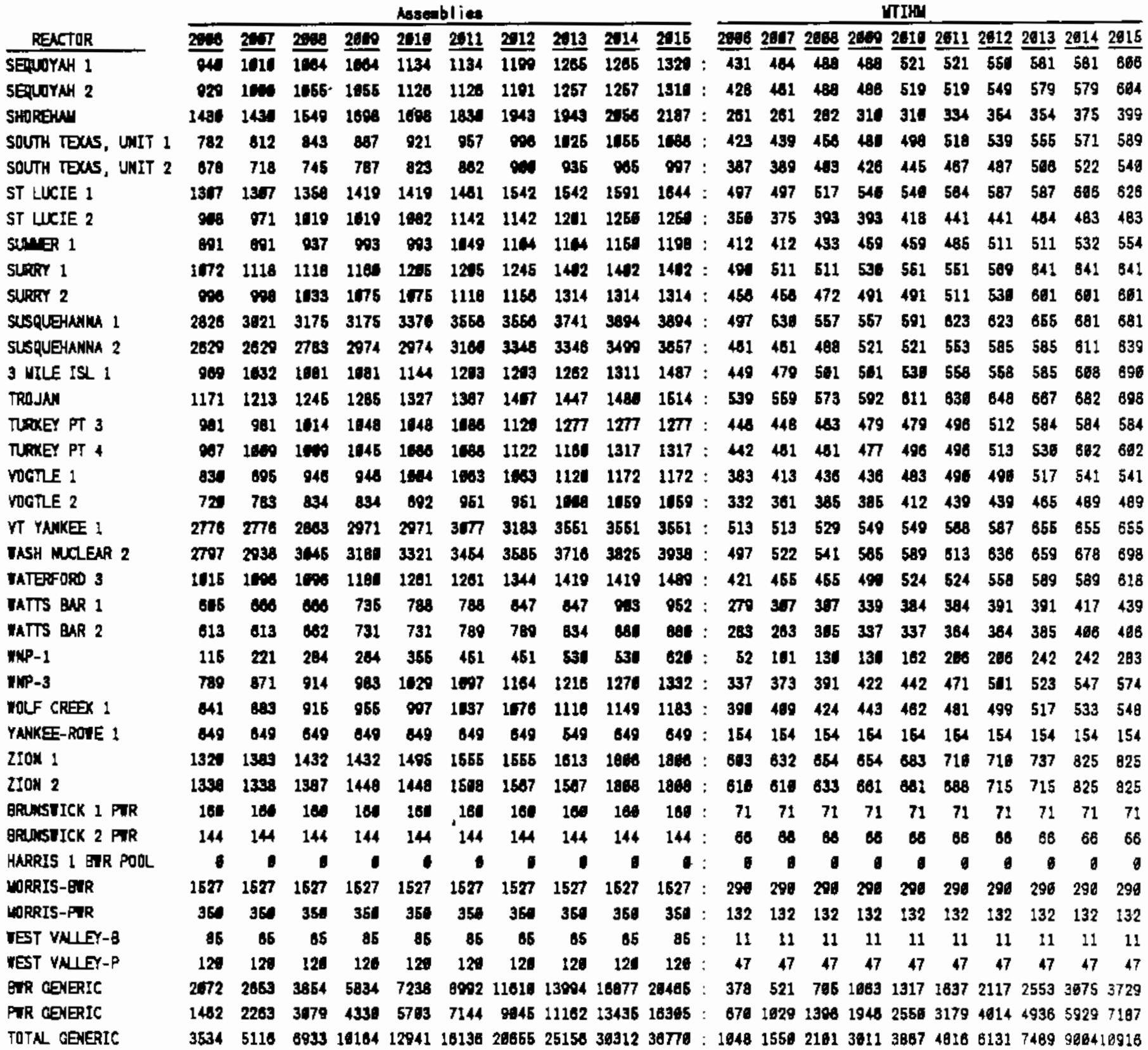


TABLE A.4. Upper Reference Case, 1985 Inventory and Projected Inventory to 2020 (contd)

\begin{tabular}{|c|c|c|c|c|c|c|c|c|c|c|}
\hline \multirow[b]{2}{*}{ REACTOR } & \multicolumn{5}{|c|}{ Assenblies } & \multicolumn{5}{|c|}{ TIV! } \\
\hline & 2018 & 2017 & $\underline{2018}$ & 2919 & $\underline{2029}$ & 2010 & 2017 & 2918 & 2019 & $\underline{292:}$ \\
\hline ARK MRENR 1 & $\overline{1359}$ & $\overline{1350}$ & $\overline{1358}$ & $\overline{1359}$ & $\overline{1350}$ & $\overline{629}$ & 820 & 620 & 820 & 828 \\
\hline ARK MREAR 2 & 1225 & 1200 & 1397 & 1397 & 1392 & 511 & 533 & 557 & 557 & 68: \\
\hline B YNEY 1 & 1610 & 1067 & 1867 & 1867 & 1607 & 698 & 768 & 768 & 780 & 708 \\
\hline B YNEY 2 & 894 & 945 & 845 & 983 & to36 & 412 & 430 & 430 & 469 & 47 \\
\hline BenLEONTE 1 & $\$ 20$ & 829 & 761 & 704 & 784 & 287 & 287 & 324 & 349 & 348 \\
\hline 8QमFONTE 2 & 725 & 725 & 793 & 865 & 855 & 331 & 331 & 362 & 390 & 39 \\
\hline BIO ROCX 1 & 658 & 568 & 558 & 560 & 558 & 73 & 73 & 73 & 73 & 73 \\
\hline BRAID.00D 1 & 1275 & 1275 & 1335 & 1379 & 1379 & 539 & 539 & 585 & 583 & 583 \\
\hline BOAIDTOOD 2 & 1222 & 1222 & 1286 & 1329 & 1389 & 517 & 517 & 64 & 582 & 582 \\
\hline BRINA FERT1 & 4628 & 4028 & 4028 & 4028 & 4028 & 245 & $B 45$ & 845 & 846 & 846 \\
\hline BROIIS FERRY2 & 4948 & 4948 & 4948 & 4948 & 4948 & 963 & 093 & 963 & 903 & 993 \\
\hline BROTLS FERTI & $4: 72$ & 4936 & 4936 & 4936 & 4938 & 761 & 899 & 890 & 899 & 899 \\
\hline BRarsTICK I & 3690 & 3590 & 3580 & 3600 & 3586 & 653 & 853 & 653 & 853 & 853 \\
\hline BRALSIICK 2 & 3980 & 3099 & 3989 & 3989 & 3989 & 744 & 744 & 744 & 744 & 744 \\
\hline BYRON 1 & 1358 & 1424 & 1495 & 1495 & 1562 & 574 & 682 & 832 & 632 & sol \\
\hline BYRON 2 & 1190 & 1248 & 1248 & 1292 & 1351 & 583 & 528 & 528 & 547 & 571 \\
\hline CNUNAY 1 & 1549 & 1618 & 1618 & 1878 & 1749 & 661 & 891 & 691 & 718 & 746 \\
\hline CALGET CIF 1 & 1865 & 1806 & 1885 & 1285 & 1865 & 707 & $7 \% 7$ & 707 & $7 n$ & $7 n$ \\
\hline CALYER aF 2 & 150 & 1671 & 1887 & 1087 & 1897 & 605 & 633 & 714 & 714 & 714 \\
\hline CATATEA I & 1316 & 1316 & 1371 & 1412 & 1463 & 557 & 557 & 580 & 597 & 619 \\
\hline CATABA 2 & 1176 & 1223 & 1223 & 1257 & 1392 & 497 & 617 & 517 & 532 & 551 \\
\hline QINTON 1 & 3468 & 340 & 3506 & 3701 & 3701 & 623 & 623 & 852 & 676 & 676 \\
\hline COUNACHE PK 1 & 1449 & 1497 & 1597 & 1679 & 1625 & Bad & 619 & 835 & 652 & 671 \\
\hline COUNCHE PX 2 & 1289 & 1336 & 1395 & 1418 & 1486 & 524 & 543 & 562 & 578 & 596 \\
\hline cotok 1 & 1942 & 1992 & 1992 & 1982 & 1992 & 81 & 881 & 681 & 861 & 861 \\
\hline $\operatorname{codK} 2$ & 1600 & 1768 & 1842 & 2935 & 2036 & 798 & 734 & 764 & 842 & 642 \\
\hline COOPER STN & 4040 & 4940 & 4440 & 4640 & 4049 & 742 & 742 & 742 & 742 & 742 \\
\hline CRYSTAL RVR 3 & 1210 & 1265 & 1442 & 1442 & 1442 & 561 & 587 & 869 & 689 & 869 \\
\hline DAYIS-BESSE : & 1170 & 1217 & 1268 & 1444 & 1444 & 546 & 578 & 594 & 657 & 657 \\
\hline DIARO CAMYON-1 & 1359 & 1440 & 1440 & \pm 511 & 1511 & 824 & $\mathbf{\infty 6 1}$ & 681 & 694 & 694 \\
\hline OIALO CAMTH-2 & $13: 2$ & 1302 & 1391 & 1391 & 1478 & 598 & 596 & 639 & 639 & 879 \\
\hline ORESCEN 1 & 683 & 283 & 883 & 863 & 683 & 76 & 74 & 70 & 76 & 70 \\
\hline DRESDEN 2 & 4350 & 4353 & 4363 & 4353 & 4353 & 751 & 751 & 751 & 751 & 751 \\
\hline ORESCEN 3 & 4335 & 4395 & 4335 & 4335 & 4335 & 748 & 740 & 748 & 746 & 748 \\
\hline DUNE APMOLD & 3722 & 3022 & 3422 & 3922 & 3822 & 543 & 543 & 543 & 543 & 543 \\
\hline ENRICO FERI-2 & 3985 & 4214 & 4214 & 4484 & 4404 & 726 & 768 & 769 & 8903 & 883 \\
\hline FNALY : & 1593 & 1758 & 1750 & 1760 & 1750 & 734 & 618 & 897 & 897 & 807 \\
\hline FAREY 2 & 1973 & 1427 & $\$ 427$ & 1473 & 1526 & 833 & 658 & 860 & 670 & 794 \\
\hline FITZPATRICK & 4393 & 4393 & 4393 & 4393 & 4393 & 792 & 792 & 792 & 792 & 792 \\
\hline FORT CNHOUN & 1110 & 1110 & 1110 & 1110 & $11 \pm 0$ & 460 & 480 & 160 & 490 & 400 \\
\hline GINM & 1143 & 1143 & 1143 & 1143 & 1143 & $46 !$ & 401 & 461 & 401 & 401 \\
\hline eruno alf : & 4445 & 4652 & 4652 & 4624 & 5038 & 788 & 825 & 925 & 856 & 892 \\
\hline GRAKD GLF 2 & 2329 & 2504 & 2797 & 2797 & 3842 & 415 & 457 & 498 & 498 & 541 \\
\hline HAOON NECK & 1445 & 1445 & 1445 & 1445 & 1445 & 595 & 595 & 596 & 595 & 595 \\
\hline HARRIS I & 803 & 839 & 889 & 869 & 946 & 373 & 398 & 494 & 494 & 421 \\
\hline HATCH 1 & 4543 & 4543 & 4543 & 4543 & 4543 & 842 & 842 & 842 & 842 & 842 \\
\hline HATCH 2 & 3637 & 3782 & 3782 & $39: 2$ & 4482 & 673 & 700 & 760 & 722 & 826 \\
\hline HOPE CREEX & 3646 & 3831 & 3631 & 3970 & 4157 & 685 & 699 & 699 & 724 & 758 \\
\hline HUMOLDT BAY & 399 & 390 & 390 & 390 & 390 & 28 & 20 & 29 & 29 & 29 \\
\hline INDIAN PT 1 & $180^{\circ}$ & 160 & 169 & 180 & 180 & 31 & 31 & 31 & 31 & $3 !$ \\
\hline
\end{tabular}


IABLE A.4. Upper Reference Case, 1985 Inventory and Projected Inventory to 2020 (contd)

\begin{tabular}{|c|c|c|c|c|c|c|c|c|c|c|}
\hline \multirow[b]{2}{*}{ RENCTOR } & \multicolumn{5}{|c|}{ Aoseablies } & \multicolumn{5}{|c|}{ प्रtIn } \\
\hline & 2016 & 2017 & 2018 & 2019 & 2020 & $\underline{2010}$ & $\underline{2617}$ & $\underline{2018}$ & 2019 & 2026 \\
\hline INDIN Pा 2 & $\overline{1680}$ & $\overline{1588}$ & $\overline{1680}$ & $\overline{1680}$ & 1688 & 718 & 718 & 718 & 718 & 718 \\
\hline INDIN PT 3 & 1604 & 1604 & 1504 & 1504 & 1594 & 729 & 728 & 728 & 728 & 728 \\
\hline KENANEX & 1387 & 1387 & 1307 & 1307 & 1367 & 523 & 523 & 523 & 523 & 523 \\
\hline LACRASSE & 546 & 548 & 546 & 546 & 546 & 73 & 73 & 73 & 73 & 73 \\
\hline USALE CTY 1 & 3878 & 3851 & 3851 & 3003 & 4187 & 676 & 791 & 701 & 727 & 759 \\
\hline LSHUE TTY 2 & 3912 & 3912 & 4888 & 4229 & 4229 & 710 & 710 & 744 & $\pi 0$ & $\pi 0$ \\
\hline LIVERICK 1 & 3513 & 3882 & 3682 & 3822 & 3004 & 627 & 657 & 667 & 691 & 712 \\
\hline LIERICX 2 & 2047 & 3145 & 3145 & 3324 & 3545 & 527 & 582 & 562 & 594 & 823 \\
\hline MAINE YNIKEI & 2908 & 2010 & 2606 & 2040 & 2006 & 759 & 759 & 759 & 759 & 759 \\
\hline KCEIRE 1 & 1403 & 1463 & 168 & 1547 & 1547 & 601 & 623 & 845 & $\mathbf{\infty 2}$ & 682 \\
\hline WCEIRE 2 & 1348 & 1398 & 1398 & $\$ 439$ & 1496 & 577 & 598 & 598 & 815 & 637 \\
\hline YIOLND-1 & 331 & 359 & 388 & 414 & 442 & 109 & 118 & 128 & 137 & 148 \\
\hline MIDCNO-2 & 701 & 753 & 897 & 897 & Bog & 324 & 349 & 374 & 374 & 398 \\
\hline UILLSTONE 1 & 4418 & 4418 & 4416 & 4418 & 4418 & 801 & 801 & atc1 & 801 & 811 \\
\hline UILSTONE 2 & 1919 & 1918 & 1919 & 1919 & 1919 & 773 & $\pi 3$ & 773 & $\pi 3$ & 773 \\
\hline MTLSTOIE 3 & 1296 & 1367 & 1357 & 1401 & 1458 & 590 & 628 & 628 & 646 & 673 \\
\hline DITICE $\perp 0$ & 2746 & 2748 & 2740 & 2746 & 2748 & 481 & 481 & 491 & 481 & 481 \\
\hline NINE UILE PT1 & 3792 & 3792 & 3792 & 3792 & 3792 & 675 & 875 & 875 & 675 & 875 \\
\hline NINE UILE PT2 & 2744 & 2746 & 2948 & 2948 & 3141 & 462 & 402 & 497 & 497 & 530 \\
\hline MRTH NMU I & 1327 & 1975 & 1631 & 1631 & 1031 & 612 & 234 & 780 & 746 & 796 \\
\hline FORTH ANMA 2 & 1240 & 1297 & 1797 & 1338 & 1387 & 576 & 590 & 500 & 617 & 841 \\
\hline DConE्土 1 & 1950 & 1868 & 1860 & 1850 & 1850 & 857 & 857 & 857 & 867 & 857 \\
\hline DCoLE 2 & 1048 & 1840 & 1849 & 1840 & 2898 & 745 & 746 & 745 & 745 & 745 \\
\hline ocaina 3 & 1537 & 1637 & 1537 & 1537 & 1597 & 712 & 712 & 712 & 712 & 712 \\
\hline ONSTER CAX 1 & 3813 & 3813 & 3813 & 3813 & 3813 & 297 & 687 & 687 & 687 & 687 \\
\hline PALISADES & 1587 & 1587 & 1507 & 1587 & 1587 & 625 & 825 & 825 & 625 & 825 \\
\hline PNLO VERDE 1 & 1636 & 2687 & 1741 & 1782 & 1833 & 798 & 730 & 754 & $\pi 2$ & 794 \\
\hline PNL VERE 2 & 1565 & 1612 & 1649 & 1899 & 1735 & 674 & 694 & 718 & 726 & 748 \\
\hline PHO VGIDE 3 & 1383 & 1428 & 1476 & 1511 & 1556 & 599 & 618 & 659 & 654 & 674 \\
\hline PEACHBOTTON 2 & 5731 & 5731 & $\$ 731$ & 5731 & 5731 & 1032 & 1932 & 1832 & 1032 & 1092 \\
\hline PEKCHBOTTOU 3 & 5554 & 5554 & 5554 & 5554 & 5654 & 1002 & 1802 & 1802 & 1062 & 1892 \\
\hline PEATY 1 & 3748 & 3920 & 4168 & 4186 & 4282 & 682 & 714 & 748 & 748 & 784 \\
\hline PGOT 2 & 3460 & 3824 & 3824 & 3827 & 4884 & 819 & 686 & 869 & 897 & 739 \\
\hline PILeqIU 1 & 3701 & $37 \theta 1$ & 3781 & 3781 & 3781 & 682 & 882 & 682 & 682 & 682 \\
\hline POINT GEACH 1 & 221.6 & 1265 & 1266 & 1296 & 1265 & 451 & 451 & 451 & 451 & 452 \\
\hline POINT BENCH 2 & 1230 & 1298 & 123: & 1230 & 1230 & 457 & 467 & 457 & 457 & 457 \\
\hline PRAIRIE ISL 1 & 1328 & 1328 & 1326 & 1326 & 1326 & 484 & 484 & 484 & 484 & 484 \\
\hline PRAIRIE ISL 2 & 1340 & 1346 & 1348 & 1348 & 1346 & 492 & 492 & 492 & 492 & 492 \\
\hline QUND CITIES 1 & 4436 & 4495 & 4435 & 4435 & 4435 & 797 & 797 & 797 & 797 & 797 \\
\hline QUAD CITIES 2 & 4611 & 4611 & 4011 & 4811 & 4611 & 829 & 829 & 829 & 829 & 829 \\
\hline SUKHO SECO-1 & 1479 & 1479 & 1479 & 1479 & 1479 & 884 & 684 & 884 & 864 & 884 \\
\hline PongINSON 2 & 1214 & 1214 & 1214 & 1214 & 1214 & 518 & 516 & 518 & 518 & 516 \\
\hline RVR BEND 1 & 3311 & 3474 & 3052 & 3852 & 3819 & 812 & 643 & 678 & 876 & 786 \\
\hline SNE WIT 2 & 1691 & 1591 & 1662 & 3717 & 1717 & 732 & 732 & 785 & 790 & 790 \\
\hline SNLEI 1 & 1697 & $\$ 789$ & 1958 & 1966 & 1968 & 779 & 869 & 898 & 998 & 898 \\
\hline SAN ONOFRE I & 706 & 765 & 785 & 765 & 785 & 283 & 283 & 283 & 283 & 283 \\
\hline SAN DNCFRE 2 & 1298 & 1349 & 1369 & 1426 & 1426 & $\$ \$ 1$ & $5 a 1$ & 581 & 6986 & 600 \\
\hline SAH ONOFRE 3 & 1298 & 1387 & 1387 & $\$ 425$ & 1425 & 559 & 581 & 581 & 805 & BBS \\
\hline SEABRDQK 1 & 1686 & 1131 & 1186 & 1214 & 1260 & 501 & 522 & 544 & 589 & 581 \\
\hline SELBRSOK 2 & 673 & 728 & $72 B$ & 784 & 843 & 311 & 336 & 338 & 382 & 389 \\
\hline
\end{tabular}


TABLE A.4. Upper Reference Case, 1985 Inventory and Projected Inventory to 2020 (contd)

\begin{tabular}{|c|c|c|c|c|c|c|c|c|c|c|}
\hline \multirow[b]{2}{*}{ RENCTOR } & \multicolumn{5}{|c|}{ Aseablies } & \multicolumn{5}{|c|}{ STIH } \\
\hline & $\underline{2016}$ & 2017 & 2018 & 2019 & $\underline{2020}$ & 2016 & $\underline{2017}$ & 2010 & 2019 & $\underline{2020}$ \\
\hline SEMOYAH 1 & 1377 & 1377 & 1443 & 1443 & 157 & 632 & 632 & 682 & 682 & 892 \\
\hline SEPMOYAH 2 & 1310 & 1372 & 1438 & 1438 & 1602 & 64 & 623 & 609 & 803 & 682 \\
\hline SHAREWH & 2187 & 2209 & 2427 & 2427 & 2550 & 39 & 418 & 442 & 442 & 467 \\
\hline SOUTH TEXAS, UNIT 1 & 1121 & 118 & 1194 & 1224 & 1259 & 87 & 628 & 244 & 802 & 681 \\
\hline SOUTH TEXAS, UNIT 2 & 1029 & 1843 & 1112 & 1130 & 1160 & 557 & 575 & $5 \%$ & 612 & 631 \\
\hline ST 山CIE 1 & 1801 & 1881 & 1801 & 1861 & 1881 & 76 & 769 & $7 \oplus$ & $7 \oplus$ & 769 \\
\hline ST LUEIE 2 & 1301 & 1957 & 1357 & 1465 & 1461 & ses & 525 & 525 & 543 & 585 \\
\hline SUER 1 & 1198 & 1251 & $\$ 317$ & 1307 & 1381 & 534 & 579 & 805 & 696 & 638 \\
\hline Supter 1 & 1402 & 1642 & 1442 & 1462 & 1442 & M1 & 641 & 41 & 841 & 841 \\
\hline SuRT 2 & 1314 & 1314 & 1314 & 1314 & 1314 & 691 & 601 & 801 & 601 & 6:1 \\
\hline SUSquEWANA 1 & 4955 & 4232 & 4232 & 4378 & 4538 & 700 & 746 & 740 & 705 & 796 \\
\hline SUSqEHANK 2 & 3857 & 3834 & $4: 24$ & 4824 & 4205 & 639 & 869 & 792 & 762 & 733 \\
\hline YILE ISL 1 & 1487 & 1487 & 1487 & 1497 & 1467 & 89 & 691 & 690 & 691 & 690 \\
\hline TRDJAN & 1540 & 1742 & 1742 & 1742 & 1742 & 714 & 803 & 803 & 803 & 893 \\
\hline TRKEY FT 3 & 1277 & 127 & 1277 & 1277 & 1277 & 584 & 584 & 584 & 584 & 584 \\
\hline TRKEY PT 4 & 1317 & 1317 & 1317 & 1317 & 1317 & 612 & 692 & 642 & 812 & 892 \\
\hline VOOLE 1 & 1224 & 1287 & 1287 & 1331 & 1389 & 585 & 594 & 594 & 614 & 641 \\
\hline YOOLE 2 & 1112 & 1174 & 1174 & 1210 & 1278 & 513 & 542 & 542 & 582 & 590 \\
\hline TT YANOKEE 1 & 3551 & 3551 & 3561 & 3551 & 3551 & 655 & 855 & 655 & 965 & 655 \\
\hline TASH MCEAR 2 & 4662 & $417 \theta$ & 4313 & 4416 & 4544 & 718 & 746 & 704 & 782 & 885 \\
\hline TATEFORD 3 & 1554 & 1554 & 1840 & 1699 & 169 & 845 & 845 & 881 & 766 & 788 \\
\hline ATTS Bar 1 & 952 & 1001 & 1811 & 1051 & 1007 & 499 & 462 & 462 & 485 & 588 \\
\hline IATTS EAR 2 & 039 & 933 & 970 & 1031 & 1031 & 436 & 430 & 452 & 476 & 478 \\
\hline INP-1 & 707 & 707 & 782 & 872 & 872 & 323 & 323 & 361 & 399 & 398 \\
\hline TMP-3 & 1391 & 1451 & $15 \%$ & 1503 & 1632 & 840 & 820 & 851 & 875 & 706 \\
\hline NOLF CRAXX 1 & 1217 & 1255 & 1295 & 1326 & 1394 & 504 & 582 & 69 & 615 & 632 \\
\hline FNKEE-POTE 1 & 049 & 849 & 040 & 849 & 849 & 154 & 154 & 154 & 154 & 154 \\
\hline ZION 1 & 1806 & 1810 & $18 \% 6$ & 1886 & 1898 & 825 & 825 & 825 & 825 & 825 \\
\hline ZION 2 & 1888 & 1899 & 1848 & 1880 & 1848 & 825 & 225 & 825 & 825 & 825 \\
\hline BUISTICK I PIR & 164 & 180 & 181 & 160 & 160 & 71 & 71 & 71 & 71 & 71 \\
\hline GanSTICX 2 PUR & 144 & 144 & 144 & 144 & 144 & 88 & 68 & 80 & 68 & 80 \\
\hline HAOAIS I ERR POOL & 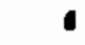 & $\bullet$ & - & 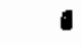 & $\bullet$ & 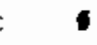 & $\theta$ & 5 & $\mathbf{1}$ & - \\
\hline VTRRI5-BUR & 1527 & 1527 & 1527 & 1527 & 1527 & 201 & 290 & 290 & 290 & 290 \\
\hline WRRIS-PIR & 36 & $35:$ & 350 & 369 & 35: & 132 & 132 & 132 & 132 & 132 \\
\hline WEST VALEY-B & 85 & 85 & 85 & 86 & 85 & 11 & 11 & 11 & 11 & 11 \\
\hline VEST YNШEY-P & 126 & 120 & 120 & 120 & 120 & 47 & 47 & 47 & 47 & 47 \\
\hline ER CEIERIC & 24495 & 29790 & 33794 & 38853 & 44347 & 4463 & 5248 & 8158 & 7878 & 8081 \\
\hline PWR GEVBIC & 19682 & 23130 & 27285 & 31563 & 35876 & 8858 & 10160 & 11971 & 13822 & 15878 \\
\hline TOTAL OENERIC & 44177 & 51935 & 61079 & 76418 & 80222 & 13119 & 15498 & 18129 & 20960 & 23759 \\
\hline
\end{tabular}


TABLE A.4. Upper Reference Case, 1985 Inventory and Projected Inventory to 2020 (contd)

SUETOTALS BY REACTOR TYPE AND TQTHLS

$\underline{1985} \quad \underline{1996} \quad \underline{1987} \quad \underline{1988} \quad \underline{1989} \quad \underline{1900} \quad \underline{1901} \quad \underline{1902} \quad \underline{1099} \quad \underline{1998} \quad \underline{1995}$

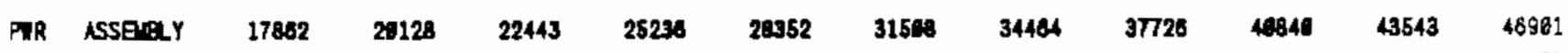

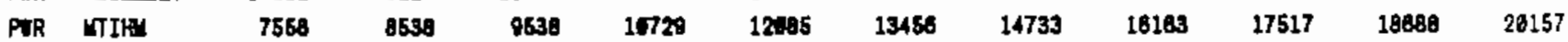

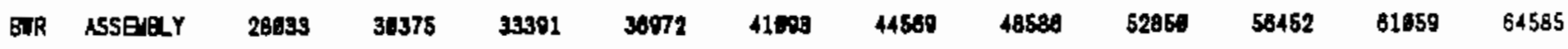

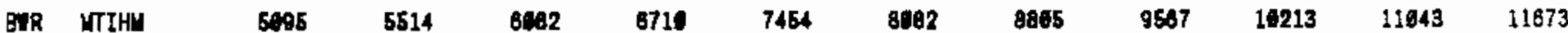

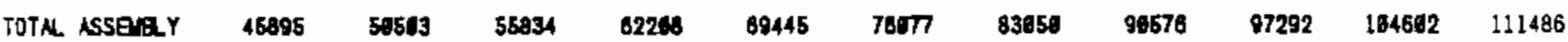

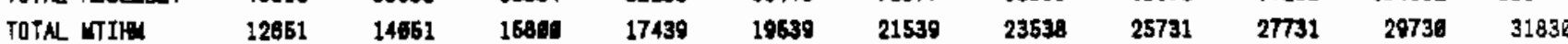

\begin{tabular}{|c|c|c|c|c|c|c|c|c|c|c|c|}
\hline & & 1996 & 1992 & $\underline{1908}$ & 1990 & 25010 & 2501 & 20192 & 2963 & $\underline{2964}$ & $\underline{2685}$ \\
\hline P & ASSERYY & 49738 & 52780 & 55797 & 58727 & 01871 & 64933 & 87760 & 78953 & 74524 & 77955 \\
\hline It & MTIH & 21397 & 2270 & 24111 & 25289 & 26891 & 28808 & 29241 & 30032 & 32298 & 33729 \\
\hline & ASSEYYY & 68889 & 72672 & 76801 & 89833 & 84711 & 88387 & 92644 & 97145 & 101329 & 105860 \\
\hline & ETIH & 12449 & 13124 & 13810 & 14531 & 15250 & 15912 & 16878 & 17480 & 18232 & 19060 \\
\hline & SSEIRYY & 118827 & $\begin{array}{r}125446 \\
35639\end{array}$ & $\begin{array}{r}132398 \\
37821\end{array}$ & $\begin{array}{r}139391 \\
39821\end{array}$ & $\begin{array}{r}146582 \\
41994\end{array}$ & $\begin{array}{r}153396 \\
43928\end{array}$ & $\begin{array}{r}181404 \\
46928\end{array}$ & $\begin{array}{r}1680065 \\
48126\end{array}$ & $\begin{array}{r}175853 \\
58439\end{array}$ & $\begin{array}{r}183555 \\
52726\end{array}$ \\
\hline
\end{tabular}

$\underline{2006} \quad \underline{2007} \quad \underline{2086} \quad \underline{2009} \quad \underline{2016} \quad \underline{2011} \quad \underline{2012} \quad \underline{2013} \quad \underline{2014} \quad \underline{2015}$

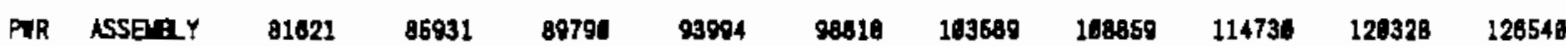

$\begin{array}{lllllllllll}\text { PTR NTIHM } & 35322 & 37222 & 38912 & 47738 & 42857 & 4915 & 47246 & 4977 & 52248 & 54958\end{array}$

$\begin{array}{lllllllllll}\text { BRR ASSEMYY II11B2 } & 115045 & 129716 & 127790 & 133871 & 146893 & 148135 & 154371 & 163961 & 172183\end{array}$

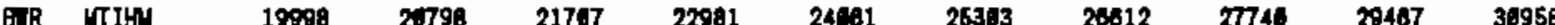

$\begin{array}{lllllllllll}\text { TOTAL NSSERYY } & 192812 & 291578 & 210586 & 221793 & 232089 & 244372 & 258904 & 260101 & 284289 & 298723\end{array}$

$\begin{array}{lllllllllll}\text { TOTAL WIIHU } & 56329 & 58629 & 60619 & 63719 & 86918 & 79218 & 73817 & 7517 & 81718 & 85918\end{array}$

\begin{tabular}{|c|c|c|c|c|c|c|}
\hline & & $\underline{2918}$ & 2017 & 2618 & 2819 & $\underline{2 \approx 28}$ \\
\hline PTR & ASSENELY & 132758 & 138978 & 145511 & 151970 & 157921 \\
\hline$P \cap \mathbb{R}$ & WTIH & 57077 & 60373 & a.3275 & 68628 & 88859 \\
\hline$B: R$ & ASSENEYY & 179275 & 187624 & 193031 & 2980501 & 299156 \\
\hline ETR & ITIHU & 32238 & 39842 & 34839 & 36286 & 378 \\
\hline ГOTA & ASSEUEL Y & $\begin{array}{r}312625 \\
89915\end{array}$ & $\begin{array}{r}325960 \\
94814\end{array}$ & $\begin{array}{r}339142 \\
99114\end{array}$ & $\begin{array}{l}352371 \\
182113\end{array}$ & 7 \\
\hline
\end{tabular}

A. 37 
TABLE A.5. Upper Reference Case, Maximum At-Reactor Capacity-Projected Annual Storage Requirements

ASSEIBIES

UTIH

\begin{tabular}{|c|c|c|c|c|c|c|c|c|c|c|c|c|c|c|c|c|c|c|c|c|}
\hline & & & & & & & & & & & & & & & & & & & & \\
\hline POOL & $\underline{1960}$ & 1997 & 1988 & $\underline{1989}$ & 1990 & $\underline{1991}$ & $\underline{1992}$ & $\underline{1993}$ & 1994 & $\underline{1995}$ & $\underline{1988}$ & $\underline{1997}$ & $\underline{1988}$ & 19891 & 1998 & $\underline{1991}$ & $\underline{1992}$ & $\underline{1993}$ & 1994 & $\underline{1995}$ \\
\hline SURRY 1I2 & 48 & $\theta$ & 82 & 93 & 44 & 51 & 94 & 43 & 44 & 94 & 22 & 0 & 30 & 43 & 28 & 23 & 43 & 20 & 28 & 43 \\
\hline PNLISAOES & 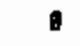 & 4 & 1 & 58 & 58 & 6 & 61 & 9 & 57 & - & 6 & 2 & 6 & 23 & 23 & 0 & 24 & $\theta$ & 22 & \\
\hline OCONEE IL & 6 & 55 & 50 & 61 & 192 & 58 & 198 & 50 & 160 & 53 & $\bullet$ & 25 & 23 & 24 & 47 & 27 & 49 & 23 & 46 & 25 \\
\hline UIUSTONE 1 & - & 86 & 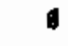 & 178 & 8 & 192 & 6 & 187 & 187 & - & 0 & 15 & 0 & 34 & $\theta$ & 34 & a & $3 a$ & 36 & $t$ \\
\hline ST LUCTE 1 & 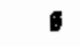 & 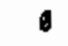 & 53 & 0 & 69 & 73 & 9 & 69 & 63 & 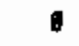 & 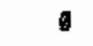 & $\sigma$ & 20 & - & 23 & 28 & 0 & 24 & 24 & 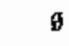 \\
\hline ROEINSON 2 & - & 0 & 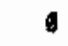 & 6 & 7 & 48 & 0 & 51 & 40 & $g$ & $\theta$ & - & a & 0 & 3 & 26 & 6 & 21 & 17 & 6 \\
\hline BRJISTICK I & a & 6 & 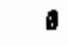 & 9 & 56 & $\theta$ & 163 & v & 155 & 183 & $\theta$ & 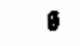 & a & 9 & 10 & 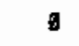 & 36 & $\theta$ & 29 & 39 \\
\hline LASNLE CTY IL2 & $\theta$ & - & 6 & 0 & 0 & 135 & 389 & 184 & 183 & 388 & $\square$ & 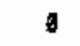 & 6 & - & 8 & 25 & 71 & 33 & 33 & 71. \\
\hline PEACHBOTTOU 2 & - & 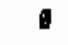 & 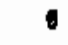 & - & 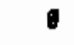 & 207 & 0 & 189 & 198 & ซ & 0 & - & 6 & 0 & 6 & 37 & 8 & 34 & 34 & $\theta$ \\
\hline PENCHEOTTOU 3 & a & 0 & 1 & $\$$ & 6 & 5 & 192 & 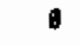 & 177 & 198 & - & 9 & d & 6 & 0 & 1 & 35 & $b$ & 31 & 34 \\
\hline PILCRIN 1 & 0 & 8 & 6 & 8 & d & $\theta$ & 88 & 6 & 105 & 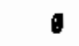 & 6 & g & - & 0 & 0 & $g$ & 12 & 日 & 29 & 9 \\
\hline CNVERT $\mathrm{CLF}$ 1:2 & 6 & g & 0 & - & 9 & 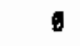 & 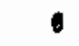 & 20 & 77 & 81 & 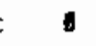 & 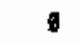 & 6 & - & 5 & 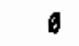 & 6 & 8 & 29 & 30 \\
\hline BRANSTICX 2 & 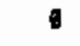 & $\theta$ & 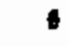 & - & 0 & • & 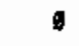 & 139 & 155 & 0 & 9 & 0 & 6 & 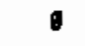 & 9 & - & 0 & 24 & 29 & $\theta$ \\
\hline OCONEE 3 & a & 8 & 0 & • & 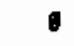 & 6 & 0 & 0 & 46 & 53 & 0 & 6 & 9 & 0 & 1 & 6 & 9 & $\sigma$ & 21 & 25 \\
\hline OYSTER CRK 1 & 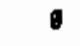 & - & • & 0 & 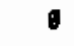 & • & 0 & 0 & 38 & - & a & 0 & 0 & 9 & d & $g$ & 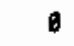 & 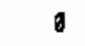 & 7 & t \\
\hline PRAIRIE ISi 122 & 0 & - & 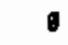 & 1 & 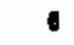 & 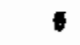 & 1 & - & 6 & 70 & 1 & 6 & 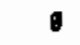 & - & - & 0 & 0 & 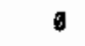 & 2 & 25 \\
\hline INDIAN PT 2 & 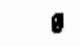 & 1 & • & $\boldsymbol{\theta}$ & 0 & $\theta$ & 0 & 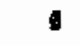 & 6 & 15 & 6 & 0 & $\bullet$ & 0 & 8 & 0 & 8 & 0 & 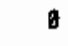 & 7 \\
\hline COCPER STN & 1 & 0 & 8 & 0 & - & - & 8 & 0 & - & 16 & 0 & • & 0 & 8 & - & 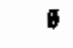 & 6 & 0 & 9 & \\
\hline PAR TOTAL. & 48 & 59 & 185 & 292 & 271 & 228 & 261 & 227 & 433 & 388 & 22 & 27 & 80 & 89 & $\$ 16$ & 98 & 118 & 95 & 183 & 154 \\
\hline BIR TOTAL & $\theta$ & 86 & 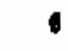 & 170 & $\$ 5$ & 539 & $B 12$ & 670 & 1230 & 751 & • & 15 & ฮ & 36 & 10 & 97 & 148 & 121 & 222 & 137 \\
\hline TOTAL & 48 & 145 & 185 & 372 & 326 & 707 & 1073 & 897 & 1683 & 1117 & 22 & 42 & 88 & 118 & 128 & 195 & 264 & 218 & 404 & 29 \\
\hline
\end{tabular}


TABLE A.5. Upper Reference Case, Maximum At-Reactor Capacity-Projected Annual Storage Requirements (contd)

ASSERIES

VIIHN

POOL

SURRY 112

PNISADES

OCONEE $1 \mathbf{M}$

UILSTONE 1

ST LUCIE :

ROBINSON 2

BRUNSIICX 1

USNUE CTY 112

PEACHBDTTEN 2

PEACHEOTIOU 3

PILGRIM 1

CALVERT QF 1 L2

BRISIICK 2

OCONE 3

OYSTER CRX 1

PRAIRIE ISL 112

INOIAN FT 2

CDOPER STN

ZION 122

BYRON 142

B VNLE 1

MISTONE 2

LIERICX 1

POINT BEACY 112

BRAIDTOD 122

BIO ROCK 1

IAINE YANXEE

FORT CNHOUN

SEQUOYAH 112

OAYIS-BESSE 1

LACROSSE

EURICD FERII-2

NINE MILE PI!

SAN OHOFRE 1,2, 13

HAOON NECK

COOK 142

FITZPATRICX

SNCEI 1

ARK RUCLAR 1

DRESDEN 2

DUANE ARMOLD

WASH MULEAR 2

PERTY 112

NORTH ANKA 112

KETALANEE

DRESDE 3

LINERICX 2

ARK WULLEAR 2

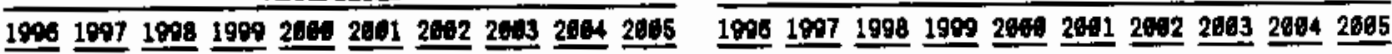

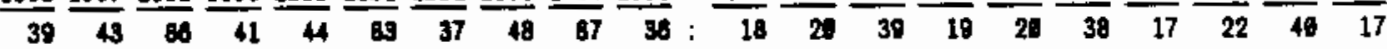

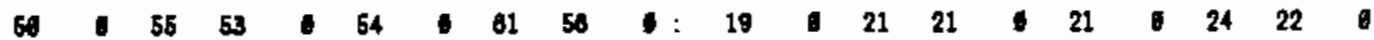

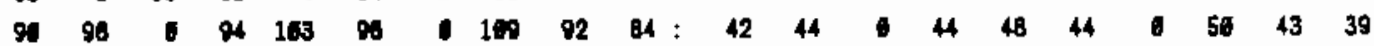

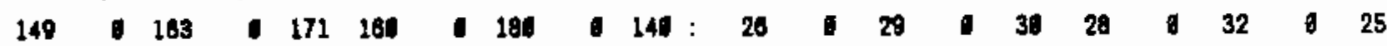

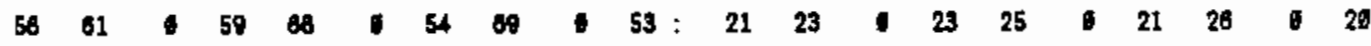

$\begin{array}{llllllllllllllllllll}38 & 48 & 3 & 37 & 41 & 1 & 43 & 44 & 49 & 0 & 16 & 26 & 0 & 18 & 18 & 0 & 18 & 19 & 17 & 0\end{array}$

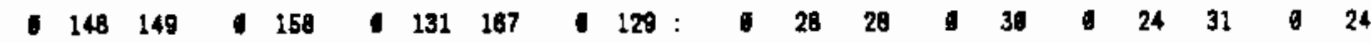

$\begin{array}{lllllllllllllllllllll}183 & 176 & 354 & 173 & 186 & 350 & 166 & 109 & 368 & 153 & 34 & 32 & 64 & 31 & 34 & 84 & 28 & 36 & 87 & 28\end{array}$

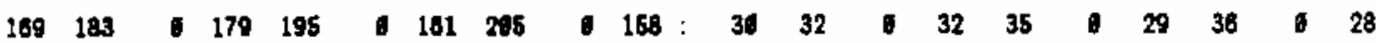

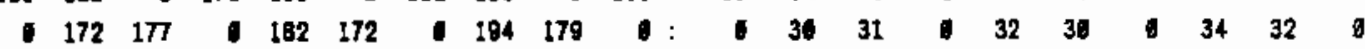

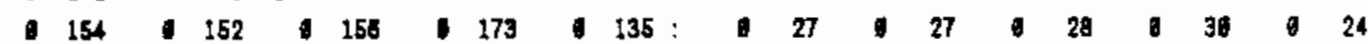

$\begin{array}{llllllllllllllllllll}69 & 73 & 74 & 72 & 78 & 72 & 95 & \text { 84 } & 76 & 84 & 26 & 27 & 29 & 27 & 29 & 27 & 24 & 32 & 29 & 24\end{array}$

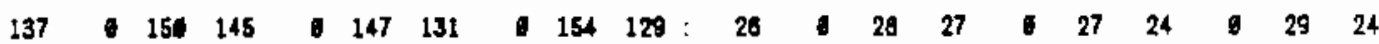

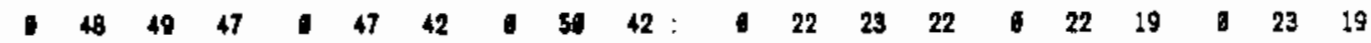

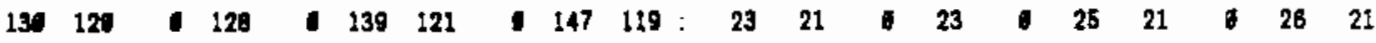

$\begin{array}{lllllllllllllllllllll}29 & 64 & 64 & 69 & 68 & 32 & 56 & 73 & 68 & 56 & : & 10 & 23 & 23 & 22 & 24 & 11 & 29 & 28 & 23 & 28\end{array}$

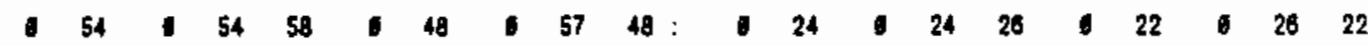

$\begin{array}{llllllllllllllllllll}86 & 96 & 94 & 87 & 96 & 86 & 79 & 161 & 92 & 76: & 16 & 16 & 17 & 16 & 17 & 16 & 14 & 18 & 17 & 14\end{array}$

$\begin{array}{lllllllllllllllllllll}32 & 58 & 58 & 113 & 11 & 57 & 1.2 & 86 & 89 & 111 & 15 & 27 & 27 & 52 & 29 & 26 & 47 & 38 & 27 & 46\end{array}$

$\begin{array}{lllllllllllllllllllll}103 & 87 & 68 & 117 & 72 & 72 & 180 & 78 & 67 & 165 & : & 44 & 28 & 29 & 49 & 38 & 36 & 46 & 32 & 28 & 44\end{array}$

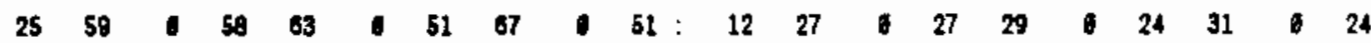

$\begin{array}{llllllllllllllllllll}27 & 59 & 5 & 1 & 82 & 58 & 1 & 68 & 69 & 0 & 11 & 24 & 24 & 6 & 25 & 24 & 0 & 27 & 24 & 9\end{array}$

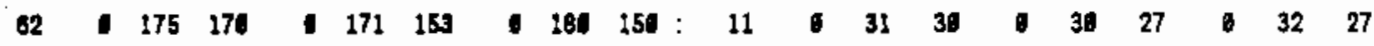

$\begin{array}{lllllllllllllllllllll}42 & 51 & 51 & 55 & 55 & 50 & 45 & 57 & 53 & 45 & : & 15 & 18 & 18 & 18 & 19 & 18 & 18 & 28 & 19 & 18\end{array}$

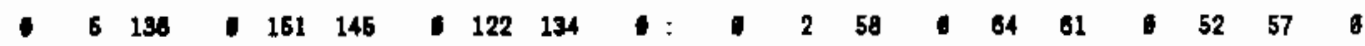

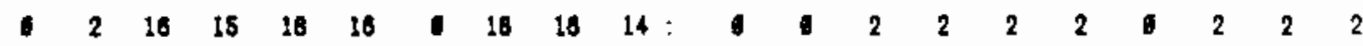

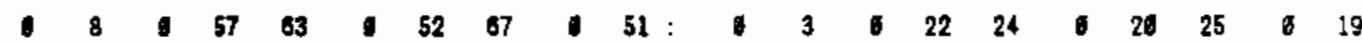

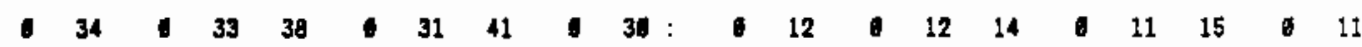

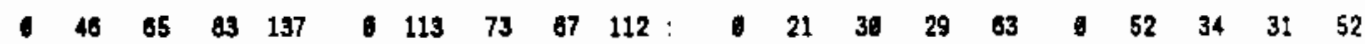

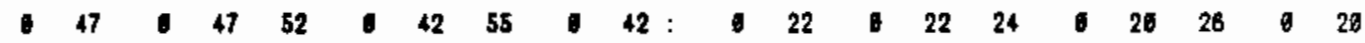

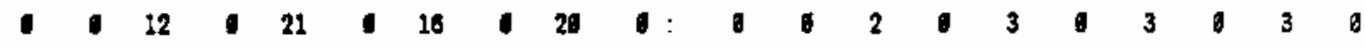

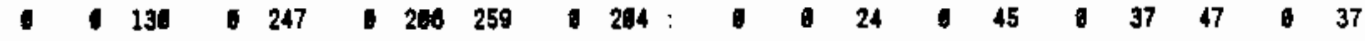

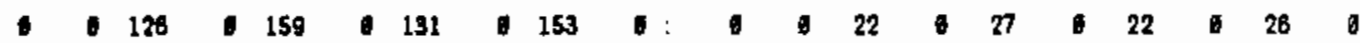

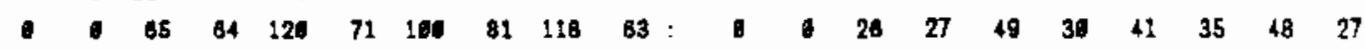

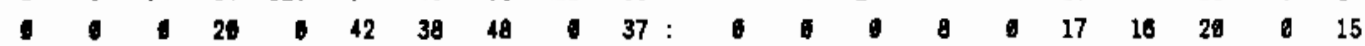

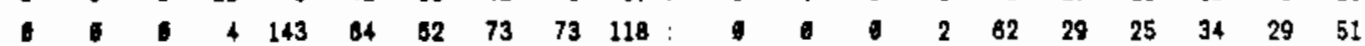

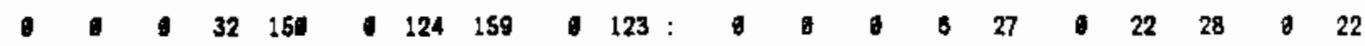

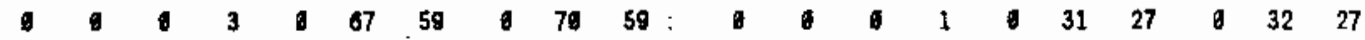

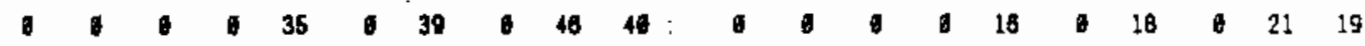

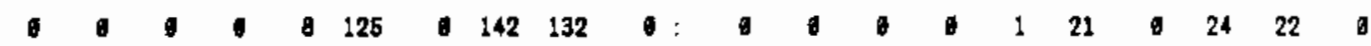

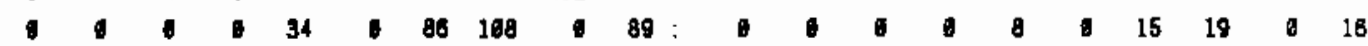

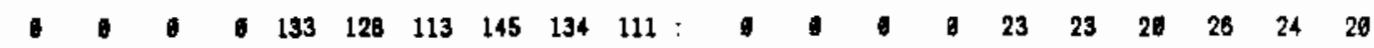

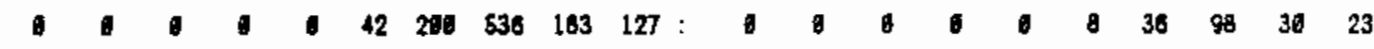

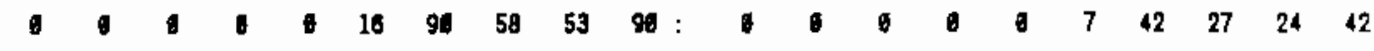

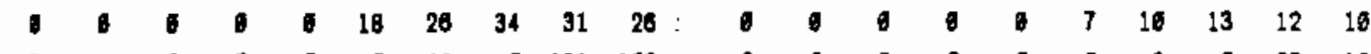

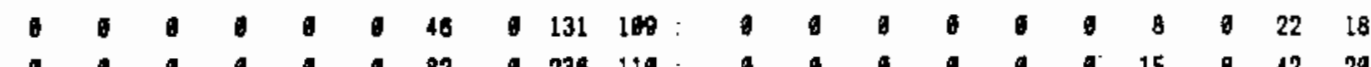

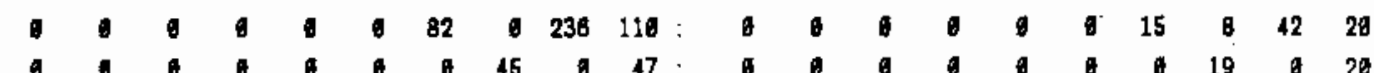


TABLE A.5. Upper Reference Case, Maximum At-Reactor Capacity-Projected Annual Storage Requirements (contd)

A5SEQIES

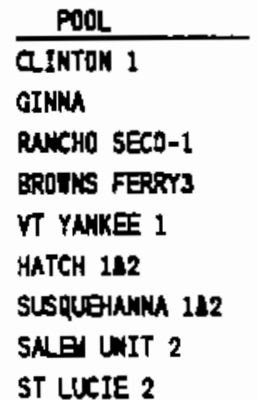

\begin{tabular}{|c|c|c|c|c|c|c|c|c|c|c|c|c|c|c|c|c|c|c|c|}
\hline 1900 & 1007 & 1998 & 1990 & 2919 & 2001 & 12002 & 2003 & 2094 & 2645 & 1896 & 1097 & 1998 & 1990 & 92860 & 2901 & 12602 & 2803 & 2964 & 202065 \\
\hline 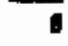 & 4 & 1 & 1 & 0 & 1 & 1 & 58 & 141 & 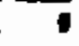 & 1 & 1 & 7 & 1 & 0 & 1 & 16 & 11 & 28 & 8 \\
\hline 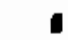 & $\bullet$ & 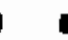 & • & . & 1 & 0 & 26 & 27 & 22 & I & • & 6 & 0 & • & . & 6 & 9 & 9 & 9 \\
\hline ป & 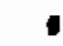 & 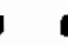 & 1 & - & 】 & 0 & 1 & 50 & 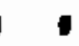 & 1 & 1 & 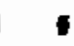 & 1 & 6 & 0 & 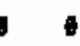 & 0 & 23 & \\
\hline 0 & $\theta$ & 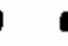 & 1 & 1 & $\theta$ & . & 47 & 1 & 160 & 1 & 6 & • & 0 & 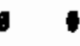 & 1 & 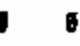 & 9 & 6 & 8 \\
\hline 0 & 0 & 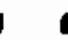 & I & 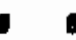 & 1 & . & 74 & 0 & 98 & 1 & E & 6 & 0 & $\theta$ & 0 & $\theta$ & 14 & 6 & 16 \\
\hline $\boldsymbol{0}$ & & 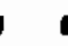 & 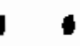 & 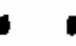 & 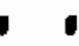 & 1 & 0 & 26 & 257 & - & 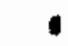 & 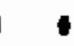 & 0 & 9 & 0 & 6 & . & 5 & 5 \\
\hline$\theta$ & 0 & 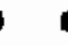 & . & . & 0 & 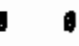 & - & 30 & 312 & 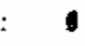 & 1 & - & 1 & 0 & $\theta$ & 8 & 0 & $\mathbf{5}$ & 5 \\
\hline 1 & ! & 6 & 6 & 6 & 1 & - & . & 68 & 5 & ๑ & . & 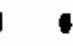 & 0 & 0 & 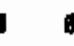 & ฮ & 0 & 31 & \\
\hline 1 & $\theta$ & 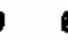 & 6 & 6 & 1 & A & 0 & 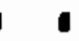 & 48 & 1 & $\theta$ & 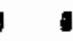 & 8 & 9 & 8 & 8 & 6 & 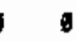 & 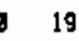 \\
\hline
\end{tabular}

PTR TOTN

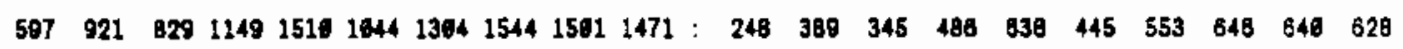
BTR TOTN

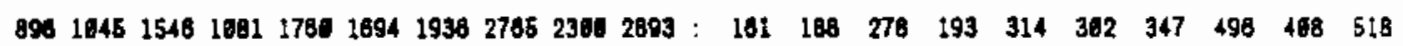
TOTN

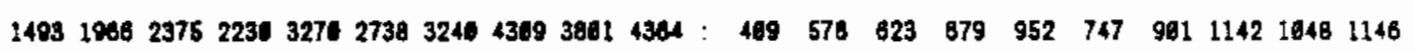


TABLE A.5. Upper Reference Case, Maximum At-Reactor Capacity-Projected Annual Storage Requirements (contd)

PODL

SLRT ILP

PNLISADES

DCONEE 162

MLLSTONE ?

ST LUCIE 1

ROBINSON 2

BRALSTICX 1

USNIE CTY $1 \mathrm{A2}$

PERCHBOTTOH 2

PEACHBOTTOU 3

PILCRIU 1

CNVERT CLF 112 BRutstick 2

OCOMEE 3

OYSIER CRK 1

PRAIRIE ISL 14

INDIAN OT 2

COOPER STN

ZION 192

BYROM 142

В พU⿴囗十 1

YILSTONE 2

LIVEICX 1

PAINT BEACH $1 \mathrm{H2}$

BRATCLOOD 112

MINE YAIKEE

FORT CNHOLN

SERLOYAH 122

DAVIS-BESSE 1

LACROSSE

EIRICO FENI-2

NINE MILE PT!

SAY ONOFRE $1,2,13$

HADDN MECX

COCK 1 L2

FITZPATAICK

SNLE 1

ARK MUCEAR 1

DRESDEN 2

DUANE ARNDLD

IASH MXEEAR 2

PERPY IL2

NORTH ANA $1: 2$

KEAUNEE

ORESDEA 3

LIUERICK 2

ARK NUCLEAR 2

GLINTON 1

\begin{tabular}{|c|c|c|c|c|c|c|c|c|c|c|c|c|c|c|c|c|c|c|c|}
\hline 108 & 27 & 2008 & 2019 & 2010 & 2011 & 2012 & 2113 & 2.1 & & 2000 & $26 \Omega I$ & 2000 & 20149 & 2010 & 2611 & 2012 & 2013 & 2014 & 15 \\
\hline 46 & 46 & 35 & 84 & 45 & 43 & 80 & 158 & & & 21 & 21 & 16 & 39 & 21 & 29 & 37 & 72 & 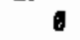 & $\theta$ \\
\hline 50 & 1 & 47 & 50 & 1 & 57 & 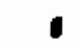 & 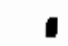 & 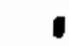 & & 23 & . & 18 & 23 & I & 22 & 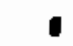 & 0 & 0 & \\
\hline 1 & 108 & 81 & 5 & 110 & 50 & 98 & 49 & 218 & & 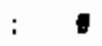 & 49 & 38 & 23 & 49 & 23 & 45 & 23 & 101 & \\
\hline 1 & 175 & 136 & 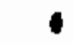 & 176 & 0 & 0 & 1 & $\bullet$ & & 1 & 31 & 24 & 1 & 31 & 6 & 1 & 8 & 6 & g \\
\hline 60 & 5 & 51 & 81 & 1 & 82 & 61 & 0 & 49 & 53 & 25 & 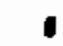 & 19 & 23 & 5 & 24 & 23 & 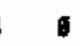 & 19 & 29 \\
\hline 52 & 42 & 1 & 51 & 42 & 50 & $\bullet$ & 1 & g & 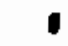 & 21 & 18 & 1 & 21 & 18 & 24 & 1 & 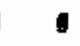 & 1 & $\theta$ \\
\hline 181 & 1 & 128 & 1 & 0 & 1 & 1 & 1 & 1 & & 34 & a & 24 & 0 & 1. & 1 & 1 & 1 & 6 & 0 \\
\hline 190 & 382 & 147 & 185 & 392 & 182 & 178 & 384 & 149 & 153 & 35 & 70 & 27 & 34 & 78 & 33 & 32 & 66 & 27 & 28 \\
\hline $2: 1$ & 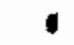 & 158 & 191 & 0 & 180 & 187 & 1 & 158 & 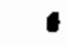 & 36 & 0 & 28 & 34 & 1 & 34 & 33 & 0 & 28 & 8 \\
\hline 368 & 180 & $\bullet$ & 181 & 189 & 1 & 177 & 177 & I & & 33 & 39 & $\boldsymbol{J}$ & 32 & 34 & 1 & 31 & 31 & 0 & 8 \\
\hline 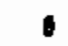 & $\bullet$ & 131 & 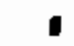 & 100 & 1 & 159 & ! & 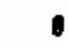 & 5 & 1 & 5 & 23 & 6 & 36 & 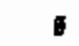 & 28 & 0 & 0 & 0 \\
\hline 81 & Bu & 82 & 75 & 81 & $n$ & 75 & 76 & 61 & 279 & 36 & 30 & 23 & 28 & 30 & 29 & 28 & 29 & 23 & 105 \\
\hline 0 & 182 & 128 & 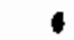 & 181 & 153 & 5 & 152 & 120 & ॥ & 0 & 34 & 24 & 0 & 30 & 29 & 0 & 28 & 24 & 8 \\
\hline 52 & 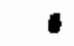 & 46 & 40 & 6 & 5 & 48 & 40 & 9 & 6 & 24 & $\bullet$ & 19 & 23 & J & 23 & 22 & 23 & 6 & 0 \\
\hline 5 & 154 & 117 & - & • & 1 & 0 & 1 & 5 & • & 0 & 27 & 21 & 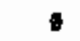 & 1 & 5 & v & 0 & 5 & 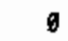 \\
\hline 30 & 70 & 64 & 85 & 71 & 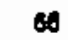 & 88 & $\infty$ & 148 & & 13 & 25 & 19 & 23 & 25 & 23 & 23 & 23 & 52 & 8 \\
\hline 1 & 80 & 1 & 55 & 61 & I & 54 & 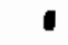 & 45 & 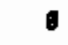 & 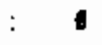 & 27 & 6 & 25 & 27 & a & 24 & 4 & 29 & 1 \\
\hline 98 & 91 & 73 & 84 & 99 & 84 & 88 & 84 & 74 & 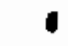 & 18 & 17 & 13 & 15 & 18 & 15 & 16 & 15 & 13 & 8 \\
\hline 84 & 63 & 98 & $B 1$ & 63 & 120 & 59 & 58 & 241 & $\theta$ & 29 & 29 & 45 & 28 & 29 & 55 & 27 & 27 & 116 & 0 \\
\hline 73 & 83 & $\infty$ & 70 & 58 & 131 & 69 & 58 & 186 & 50 & 31 & 27 & 42 & 39 & 25 & 55 & 29 & 24 & 45 & 25 \\
\hline 63 & 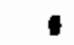 & 49 & 61 & 84 & 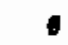 & 58 & 66 & 9 & 52 & 29 & 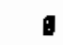 & 23 & 28 & 30 & 9 & 27 & 28 & $\theta$ & 24 \\
\hline 84 & 68 & 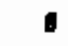 & 59 & 84 & 6 & 58 & 50 & 1 & 0 & 26 & 26 & 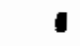 & 24 & 6 & 5 & 24 & 24 & 6 & 26 \\
\hline 0 & 187 & 146 & $\mathbf{0}$ & 180 & 179 & 1 & 17 & 147 & 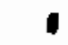 & v & 39 & 23 & 6 & 39 & 32 & 0 & 31 & 26 & 0 \\
\hline 57 & 55 & 4 & 6 & 57 & 140 & 25 & 6 & - & 1 & 21 & 20 & 16 & 16 & 21 & 52 & 9 & 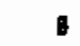 & $\theta$ & 6 \\
\hline 131 & 128 & 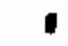 & 127 & 116 & - & 128 & 112 & $\bullet$ & 108 & 55 & 54 & $\bullet$ & 54 & 49 & 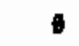 & 53 & 47 & 6 & 44 \\
\hline 63 & 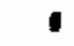 & S1) & 58 & 6 & 68 & 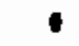 & 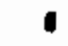 & 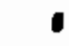 & | & 24 & 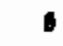 & 19 & 22 & 0 & 23 & 0 & 0 & $\theta$ & $g$ \\
\hline 39 & 1 & 29 & 36 & - & 30 & 34 & 1 & • & 1 & 14 & $\theta$ & 10 & 13 & 1 & 13 & 12 & 1 & 0 & $\theta$ \\
\hline 1 & 141 & 100 & 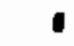 & 141 & 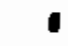 & 1310 & 132 & 0 & 188 & 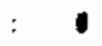 & 65 & 5. & 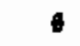 & 65 & 0 & 89 & 81 & 0 & 56 \\
\hline 53 & ๑ & 41 & 5 & 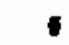 & 5 & E5 & 1 & 41 & 42 & 25 & 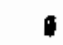 & 19 & 23 & 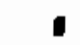 & 23 & 23 & 0 & 19 & 19 \\
\hline 21 & 0 & 16 & & 1 & & 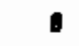 & 6 & & & & & 3 & 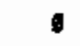 & 1 & 9 & 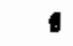 & $\theta$ & 0 & 0 \\
\hline $\mathbf{1}$ & 252 & 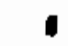 & 247 & 252 & $\bullet$ & 230 & - & 107 & & 4 & 40 & 1 & 46 & 48 & 6 & 43 & 1 & 36 & 6 \\
\hline 161 & $\bullet$ & 127 & 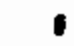 & 150 & 1 & 6 & 6 & 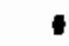 & 6 & 28 & 0 & 22 & 0 & 27 & g & 0 & I & 0 & 0 \\
\hline 261 & 1 & 157 & 231 & 76 & 75 & 73 & 74 & 82 & 63 & 83 & 0 & 65 & 90 & 33 & 32 & 31 & 32 & 26 & 27 \\
\hline 46 & 46 & $\boldsymbol{\Delta}$ & - & 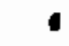 & ๑ & 1 & ॥ & $\bullet$ & 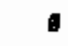 & 19 & 19 & $b$ & 0 & 6 & 6 & 0 & 9 & 0 & 0 \\
\hline 69 & 70 & 115 & 97 & 77 & 60 & 72 & 138 & 54 & 253 & 32 & 31 & 50 & 31 & 31 & 30 & 29 & 59 & 25 & 113 \\
\hline 153 & $\theta$ & 122 & 146 & a & 140 & 142 & 0 & 120 & 122 & 27 & 0 & 22 & 26 & $\theta$ & 26 & 25 & 0 & 21 & 22 \\
\hline 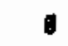 & 74 & 57 & 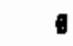 & 74 & 70 & 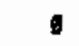 & 89 & 56 & $\theta$ & $\theta$ & 34 & 28 & H & 34 & 32 & 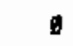 & 32 & 27 & 0 \\
\hline 0 & 54 & 6 & 47 & 6 & 47 & 47 & 6 & 39 & (1) & 6 & 23 & 0 & 22 & 0 & 22 & 22 & 0 & 18 & $\theta$ \\
\hline 137 & 137 & 0 & 135 & a & 0 & 6 & 6 & $\theta$ & b & 23 & 23 & 9 & 23 & 8 & 0 & D & 0 & 0 & 0 \\
\hline 118 & 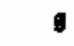 & 82 & 99 & 0 & 100 & $\infty$ & פ & 83 & 86 & 19 & $\theta$ & 15 & 18 & - & 16 & 18 & 0 & 15 & 15 \\
\hline 139 & 139 & 100 & 135 & 141 & 133 & 131 & 131 & 108 & 11 & 24 & 24 & 19 & 24 & 25 & 23 & 23 & 23 & 19 & 28 \\
\hline 499 & 140 & 165 & 459 & 101 & 232 & 430 & 137 & 211 & 150 & 91 & 27 & 30 & 83 & 29 & 42 & 78 & 25 & 38 & 27 \\
\hline 55 & 58 & 87 & d & 113 & 186 & 0 & 160 & 84 & 0 & 25 & 26 & 40 & 4 & 52 & 49 & 0 & 48 & 39 & 0 \\
\hline 33 & 33 & 25 & 36 & 32 & 31 & 31 & 29 & 25 & & 13 & 13 & 9 & 11 & 12 & 12 & 12 & 11 & 9 & g \\
\hline 1 & $\$ 37$ & 107 & . & 137 & 131 & $\theta$ & 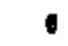 & ต & & $\theta$ & 23 & 18 & 0 & 23 & 22 & b & g & $\theta$ & 0 \\
\hline 1 & 255 & 1 & 227 & 158 & 6 & 210 & $B$ & 183 & 294 & $\theta$ & 45 & 0 & 40 & 28 & 6 & 38 & 6 & 32 & 36 \\
\hline 0 & 59 & 47 & 6 & 59 & 6 & 55 & 57 & 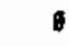 & 45 & 0 & 24 & 29 & 1 & 24 & 1 & 23 & 24 & $\theta$ & 19 \\
\hline 189 & 123 & & 175 & 138 & & 152 & 119 & & 139 & 33 & 22 & $g$ & 32 & 25 & 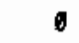 & 28 & 22 & 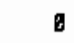 & 24 \\
\hline
\end{tabular}


IABLE A.5. Upper Reference Case, Maximum At-Reactor Capacity-Projected Annual Storage Requirements (contd)

ASSBALIES

VTILA

POOL

arNM

RUCHO SECO-1

ERDThS FERTrY3

VT YANKEE 1

HATCH ILO

SLSquEHANK 142

SNEA UNIT 2

ST UCYE 2

PNLO VERDE 1

PNDO VEDE 2

TURXEY PT IL2

BROTIN FERRYLL

IATTS BAR 1A2

FARLEY 1

CRISTNL RVR 3

VOGTLE 112

TROJAN

INOIAN PT 3

CHATAY 1

RVR EQVD 1

WATERFORD 3

DONTCELO

PNLO VEDE 3

QUAD CITIES $1: 2$

DIARO CANYON-1

NNP-3

DIAQLO CAKYON-2

FAREY 2

rCUIRE 1

HOPE CREEX

WOLF CAEEX 1

SEABROOK :

SUMER 1

CONAKCHE PK 112

WCUIRE 2

SHOREIAN

PUR TOTN

GTR TOTN

TOTN L

\begin{tabular}{|c|c|c|c|c|c|c|c|c|c|c|c|c|c|c|c|c|c|c|c|c|}
\hline 608 & 2007 & 2008 & 2000 & 2010 & 2011 & & & & 2.16 & & 2000 & $2[\pi$ & 2008 & 2000 & 2010 & 2011 & 212 & 2013 & 2614 & 2615 \\
\hline 20 & 28 & 22 & 28 & 28 & & & & & & & 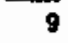 & 8 & 7 & & 0 & 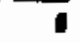 & 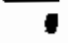 & - & & \\
\hline 51 & 54 & 0 & 51 & 58 & 1 & 50 & 51 & & 41 & & 24 & 25 & & 24 & 23 & 1 & 23 & 24 & & 19 \\
\hline 211 & 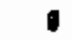 & 158 & 5 & 261 & 190 & 1 & 188 & 1 & 158 & & 36 & 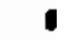 & 28 & & 36 & 34 & 0 & 34 & & 29 \\
\hline 112 & 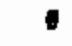 & 87 & 180 & • & 100 & 160 & 0 & 0 & 0 & & 11 & 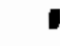 & 18 & 21 & 1 & 10 & 19 & & & \\
\hline 162 & 150 & 250 & 152 & 164 & 344 & 150 & 152 & 216 & 0 & & 31 & 31 & 46 & 28 & 30 & 58 & 28 & 28 & 150 & \\
\hline 197 & 195 & 3A & 191 & 195 & 372 & 188 & 185 & 300 & 158 & & 34 & 34 & 53 & 39 & 34 & 04 & 32 & 32 & 53 & 27 \\
\hline 73 & 73 & 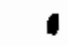 & 71 & 74 & 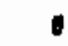 & 48 & 89 & 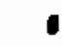 & 58 & & 34 & 34 & 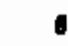 & 39 & 34 & 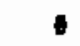 & 31 & 33 & 1 & 27 \\
\hline 1 & 63 & 48 & 1 & 63 & 61 & 0 & 50 & 49 & 1 & & 1 & 25 & 19 & 0 & 25 & 23 & 8 & 23 & 19 & B \\
\hline 41 & 56 & 43 & 54 & 50 & 54 & 53 & 54 & 45 & 46 & & 18 & 24 & 18 & 24 & 24 & 24 & 23 & 24 & 28 & 20 \\
\hline 44 & 36 & 41 & 55 & 43 & 48 & 48 & 36 & 40 & 42 & & 19 & 17 & 16 & 24 & 18 & 24 & 21 & 16 & 17 & 18 \\
\hline 85 & 42 & 39 & 74 & 41 & 38 & 76 & 196 & 1 & 0 & & 30 & 19 & 15 & 32 & 19 & 17 & 32 & 98 & & a \\
\hline 100 & 0 & 311 & 383 & 1 & 370 & - & 376 & 312 & 764 & & 20 & 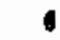 & 50 & 79 & $\theta$ & 59 & 8 & 68 & 57 & 139 \\
\hline$\infty$ & of & 49 & 138 & 53 & 58 & 50 & 45 & 102 & 49 & & 46 & 28 & 23 & 64 & 24 & 27 & 27 & 21 & 47 & 23 \\
\hline 1 & 4 & 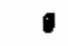 & 64 & 81 & - & 58 & 80 & 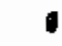 & 48 & & 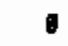 & 2 & 0 & 28 & 26 & ฮ & 27 & 28 & & 22 \\
\hline 1 & 3 & 0 & 61 & 1 & 59 & 1 & 60 & 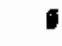 & 48 & & 1 & 1 & 6 & $2 B$ & 1 & 27 & 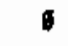 & 28 & $\theta$ & 22 \\
\hline - & 1 & 101 & 1 & 116 & 118 & 0 & 114 & 103 & 5 & & 1 & 4 & 47 & 1 & 54 & 54 & ฮ & 53 & 48 & $b$ \\
\hline$\bullet$ & 0 & 31 & 40 & 42 & 40 & 40 & 40 & 39 & 34 & & 0 & 0 & 14 & 18 & 19 & 18 & 16 & 18 & 15 & 16 \\
\hline 9 & 0 & 35 & 1 & 67 & 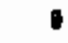 & 61 & 82 & ॥ & 52 & & 1 & $\pi$ & 18 & 6 & 31 & 0 & 28 & 28 & 6 & 24 \\
\hline 0 & 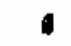 & 38 & 1 & 84 & 73 & 0 & 86 & 60 & 0 & & ๑ & 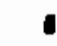 & 10 & 0 & 36 & 31 & 0 & 34 & 25 & $\theta$ \\
\hline 1 & 1 & 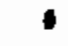 & 131 & ๑ & 173 & 171 & 1 & 142 & 140 & & 1 & 1 & 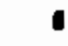 & 24 & 6 & 32 & 32 & 0 & 26 & 27 \\
\hline 1 & ๑ & 6 & 31 & 81 & - & 83 & 75 & ฮ & 70 & & 1 & • & ॥ & 13 & 34 & 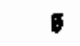 & 34 & 31 & 6 & 29 \\
\hline 5 & 1 & 1 & 44 & a & 91 & 0 & 0 & ๑ & 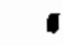 & & 1 & 0 & 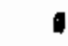 & 8 & 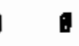 & 18 & 6 & 0 & 6 & 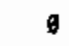 \\
\hline 1 & 1 & 1 & 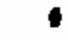 & 34 & 48 & 48 & 45 & 30 & 41 & & 0 & 1 & $\theta$ & 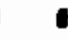 & 15 & 21 & 21 & 29 & 17 & 18 \\
\hline - & 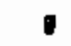 & ฮ & 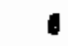 & 248 & 134 & 133 & 265 & 712 & ॥ & & 5 & $\theta$ & 0 & 0 & 44 & 24 & 23 & 47 & 125 & 6 \\
\hline 1 & 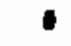 & $\theta$ & 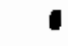 & $\bullet$ & 67 & 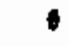 & 85 & 0 & 78 & & 6 & 0 & 6 & 0 & 6 & 31 & $\theta$ & 39 & 1 & 35 \\
\hline 0 & 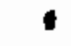 & 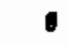 & 4 & 6 & 9 & 67 & 52 & 54 & 82 & & 6 & 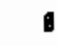 & 0 & 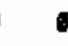 & $b$ & 4 & 29 & 23 & 24 & 27 \\
\hline 0 & $\bullet$ & 1 & I & $\bullet$ & 4 & 24 & 6 & 70 & 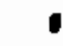 & & 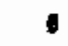 & 1 & $\theta$ & I & 1 & 0 & 11 & 0 & 32 & 0 \\
\hline$B$ & 1 & 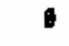 & . & - & 4 & - & 24 & 47 & : & : & 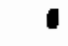 & 1 & 1 & 8 & 9 & g & 0 & 11 & 22 & $\theta$ \\
\hline 0 & 1 & 6 & - & - & c & $\theta$ & 45 & 44 & 44 & : & - & $\theta$ & - & 8 & . & 6 & 0 & 19 & 19 & 19 \\
\hline 5 & 1 & 1 & D & 0 & 0 & 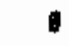 & 117 & 155 & 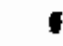 & : & 0 & 6 & 1 & 1 & • & 6 & 0 & 21 & 28 & 0 \\
\hline ฮ & 1 & 1 & • & . & $\bullet$ & 1 & 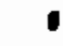 & 2 & 34 & : & 1 & 8 & 1 & 1 & 1 & 6 & 0 & 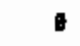 & 1 & 16 \\
\hline - & 1 & 1 & 4 & • & 6 & 6 & - & 2 & - & & 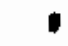 & 0 & 0 & 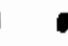 & 1 & - & 0 & $\theta$ & 1 & $\theta$ \\
\hline 6 & 1 & 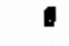 & . & 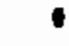 & • & 6 & 6 & 31 & 48 & : & 1 & 8 & 8 & 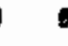 & 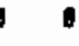 & c & $\theta$ & $\theta$ & 14 & 22 \\
\hline 0 & 1 & 0 & 」 & • & $t$ & 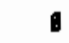 & 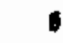 & 30 & 84 & & B & 6 & $\theta$ & 8 & D & 0 & 0 & D & 15 & 34 \\
\hline - & 1 & - & - & 1 & 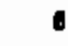 & ๑ & $\bullet$ & $\theta$ & 32 & & & 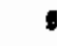 & $\theta$ & 8 & 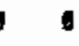 & 1 & 0 & 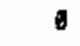 & B & 14 \\
\hline ด & 5 & 6 & $\theta$ & - & 1 & 8 & 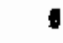 & 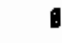 & 62 & & 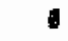 & 1 & 1 & 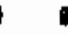 & g & $\theta$ & 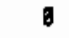 & 0 & $\theta$ & 11 \\
\hline
\end{tabular}

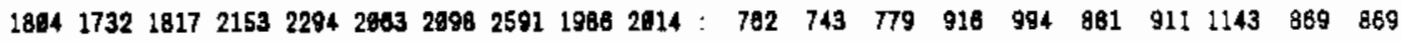

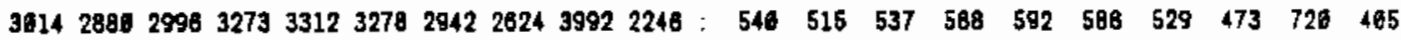

$4818461248155428580653415649521559864280: 1383125913181584158614691446161815891274$ 
TABLE A.5. Upper Reference Case, Maximum At-Reactor Capacity-Projected Annual Storage Requirements (contd)

\begin{tabular}{|c|c|c|c|c|c|c|c|c|c|c|}
\hline & & & & & & & & & & \\
\hline POLL & $\underline{2018}$ & 2017 & $\underline{2518}$ & 2619 & $\underline{2020}$ & 2918 & 2017 & $\underline{2018}$ & 2019 & 2620 \\
\hline LASNUE CTY 112 & $\overline{315}$ & 173 & $\overline{188}$ & $\overline{203}$ & $\overline{174}$ & 57 & 31 & 34 & 52 & 32 \\
\hline CNVERT af 112 & 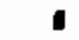 & 72 & $\mathbf{0}$ & 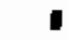 & 1 & - & 27 & 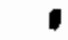 & 0 & 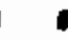 \\
\hline BMaN 12 & 52 & 124 & 71 & 4 & 128 & 22 & 52 & 39 & 19 & 53 \\
\hline 8 YNEY 1 & 53 & $\bullet$ & $\leftarrow$ & $\bullet$ & 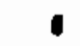 & 24 & $\boldsymbol{0}$ & 1 & $\bullet$ & 8 \\
\hline LIYERICK 1 & 154 & 168 & 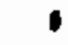 & 146 & 172 & 27 & 30 & a & 25 & 30 \\
\hline BRAIOTO00 112 & 164 & 6 & 124 & 87 & - & 4 & 6 & 52 & 37 & $\theta$ \\
\hline SE耳ОYAH 112 & 57 & 62 & 132 & $\theta$ & 128 & 28 & 20 & 61 & 6 & 59 \\
\hline DAVIS-8ESEE 1 & - & 47 & 51 & $\theta$ & 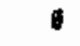 & 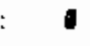 & 22 & 24 & $\theta$ & 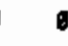 \\
\hline ENRICO FERII-2 & 212 & 228 & - & 190 & - & 39 & 42 & 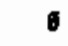 & 35 & \\
\hline SAN ONOFRE $1,2,23$ & 64 & 142 & 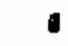 & 115 & 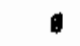 & 27 & 81 & a & 49 & 9 \\
\hline $\operatorname{cook} 112$ & 1 & 69 & 74 & 1 & 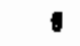 & 0 & 28 & 30 & 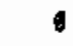 & 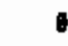 \\
\hline SALE 1 & 86 & 88 & 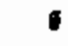 & 6 & 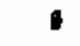 & $2 B$ & 39 & $\theta$ & 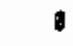 & 6 \\
\hline TASH MCLEAR 2 & 114 & 126 & 135 & 103 & 128 & 20 & 22 & 24 & 18 & 23 \\
\hline PERTI 122 & 218 & 308 & 188 & 293 & 40 & 40 & 72 & 34 & 37 & 74 \\
\hline NORTH ANA $1 \$ 2$ & 98 & $\boldsymbol{8}$ & 150 & 39 & $\mathbf{5 1}$ & 41 & 44 & 72 & 18 & 24 \\
\hline LIIERICK 2 & 6 & 198 & $\bullet$ & 170 & 221 & $\bullet$ & 35 & s & 32 & 39 \\
\hline APA MCLER 2 & : & 55 & 57 & 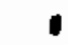 & 56 & $\mathbf{0}$ & 23 & 24 & t & 23 \\
\hline CLINTON 1 & 142 & $\boldsymbol{1}$ & 150 & 136 & $\bullet$ & 26 & - & 29 & 25 & 6 \\
\hline BRouls FERRT3 & 154 & - & $\bullet$ & ฮ & 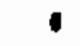 & 36 & 6 & - & 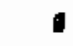 & 9 \\
\hline MATCH 112 & 132 & 145 & 0 & 126 & - & 25 & 27 & 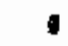 & 22 & - \\
\hline SLSqUEMNM 1 12 & 181 & 354 & 190 & 140 & 391 & 28 & 81 & 33 & 25 & 52 \\
\hline SNEI WIIT 2 & 86 & 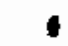 & 71 & 55 & $\bullet$ & 28 & 6 & 33 & 25 & 0 \\
\hline st WUCIE 2 & 51 & 5 & 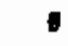 & 10 & 57 & 20 & 22 & - & 18 & 22 \\
\hline PNLO VEOE 1 & 47 & 51 & 54 & 41 & 51 & 21 & 22 & 24 & 18 & 22 \\
\hline PALO VEROE 2 & 44 & 47 & 37 & 41 & 45 & 19 & 21 & 18 & $1 B$ & 2 \\
\hline IATTS BAR 112 & 53 & 49 & 48 & 102 & 48 & 24 & 23 & 21 & 47 & 21 \\
\hline FAREY 1 & 52 & 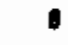 & 6 & - & 6 & 24 & 0 & 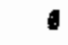 & 9 & \\
\hline CRISTNL RVR 3 & 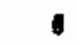 & 55 & $\bullet$ & 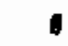 & 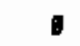 & 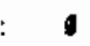 & 25 & $\theta$ & 0 & 8 \\
\hline VGTLE IL2 & 195 & 126 & 」 & 89 & 117 & 46 & 58 & 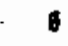 & 41 & 54 \\
\hline TROJAN & 36 & $\bullet$ & 6 & 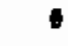 & 1 & 18 & 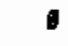 & $\boldsymbol{1}$ & 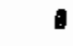 & 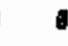 \\
\hline CNUATAY ! & 67 & $\infty$ & . & $\infty$ & 71 & 28 & 29 & 与 & 25 & 39 \\
\hline $\operatorname{RNR} \mathrm{BOO} 1$ & 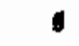 & 163 & 178 & 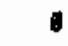 & 167 & - & 36 & 33 & 6 & 31 \\
\hline IATEFORD 3 & 85 & 1 & 2 & 59 & - & 27 & - & 36 & 25 & $\theta$ \\
\hline PNO VEROE 3 & 40 & 46 & 48 & 36 & 46 & 17 & 29 & 21 & 15 & 20 \\
\hline OIAECD CAMYON-1 & 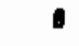 & 81 & 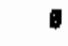 & 71 & 8 & a & 37 & 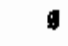 & 33 & 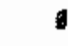 \\
\hline INP-3 & 59 & 80 & 58 & $\$ 4$ & 69 & 28 & 26 & 25 & 24 & 30 \\
\hline OIAECO CANYON-2 & 77 & 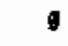 & 89 & 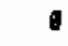 & 87 & 35 & 0 & 41 & $\theta$ & 48 \\
\hline FARLEY 2 & 52 & 54 & 9 & 48 & 55 & 24 & 25 & 6 & 21 & 25 \\
\hline LCEIRE 1 & $\theta$ & 50 & 53 & 41 & 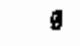 & $\theta$ & 21 & 22 & 17 & $\theta$ \\
\hline HOPE CREEX & 164 & $\$ 83$ & 0 & 139 & 187 & 36 & $\mathbf{3 3}$ & 6 & 25 & 34 \\
\hline TDF CREXX 1 & 34 & 38 & 40 & 31 & 38 & 16 & 18 & 19 & 14 & 18 \\
\hline SEABROCK 1 & 41 & 46 & 49 & 34 & 40 & 19 & 21 & 23 & 16 & 21 \\
\hline SL:PR 1 & 0 & 53 & 56 & 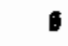 & 54 & H & 25 & 20 & 0 & 25 \\
\hline COWUCHE PK 182 & 83 & 95 & 89 & 78 & 93 & 33 & 38 & 36 & 31 & 37 \\
\hline ICEUIRE 2 & 46 & se & b & 41 & 51 & 19 & 21 & 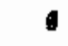 & 17 & 22 \\
\hline SHOREYAU & 6 & 190 & 134 & 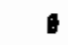 & 132 & 0 & 19 & 24 & 0 & 24 \\
\hline GRAND CUF 1 & 97 & 207 & 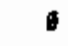 & 172 & 212 & 17 & 36 & 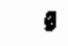 & 30 & 37 \\
\hline B VALEY 2 & 6 & 14 & 6 & 38 & 52 & $\theta$ & 6 & 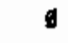 & 18 & 24 \\
\hline
\end{tabular}


TABLE A.5. Upper Reference Case, Maximum At-Reactor Capacity-Projected Annual Storage Requirements (contd)

POOL

B YNLYY 2

BUFONTE 2

PNR TOTN

ETR TOTAL

TOTN
ISSEMLIES

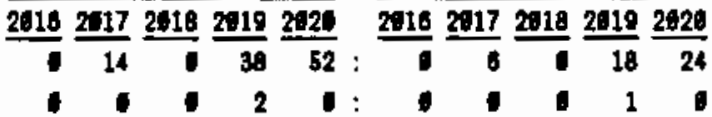

$\begin{array}{llllllllll}1480 & 1779 & 1441 & 1247 & 1337 & 858 & 774 & 034 & 546 & 590\end{array}$

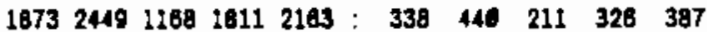

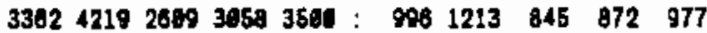


TABLE A.6. Upper Reference Case, Maximum At-Reactor Capacity-Projected Cumulative Storage Requirements

ASSEMIES

บTIH!

POOL

\begin{tabular}{|c|c|c|c|c|c|c|c|c|c|}
\hline 286 & 1097 & 1098 & 1989 & 1990 & 1901 & 1992 & 1903 & 100 & 1905 \\
\hline 48 & 48 & 130 & 225 & 207 & 318 & 412 & 465 & 400 & 593 \\
\hline 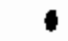 & 4 & 4 & 62 & 121 & 121 & 101 & 181 & 238 & 239 \\
\hline 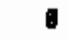 & 55 & 106 & 158 & 258 & 316 & 422 & 472 & 572 & 625 \\
\hline 0 & 20 & 80 & 250 & 256 & 446 & 448 & 615 & 782 & 782 \\
\hline 5 & 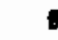 & 63 & 56 & 113 & 180 & 186 & 248 & 912 & 312 \\
\hline 6 & 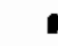 & $\theta$ & 8 & 7 & 53 & 59 & 104 & 144 & 144 \\
\hline 0 & 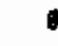 & 1 & 8 & 55 & 56 & 218 & 218 & 373 & 636 \\
\hline 0 & 6 & 1 & 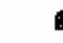 & I & 135 & 524 & 780 & 801 & 1279 \\
\hline 0 & 1 & - & 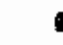 & 6 & $2 \pi$ & 207 & 390 & 580 & 596 \\
\hline 1 & 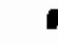 & 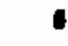 & 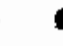 & ) & 5 & 197 & 197 & 374 & 584 \\
\hline 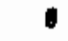 & 1 & $\bullet$ & 1 & 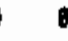 & 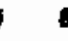 & 68 & 88 & 233 & 233 \\
\hline a & 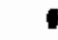 & 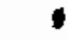 & 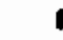 & 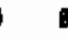 & 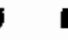 & 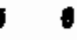 & 20 & 97 & 178 \\
\hline ฮ & 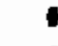 & ^ & 1 & 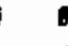 & I & & 130 & 286 & 285 \\
\hline 4 & 6 & 1 & 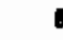 & & 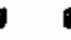 & 1 & 1 & 46 & 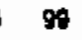 \\
\hline 6 & 1 & 5 & 8 & 6 & 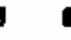 & - & 0 & 38 & 38 \\
\hline 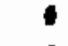 & - & 6 & 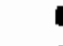 & I & 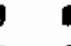 & 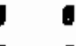 & $\theta$ & b & 76 \\
\hline 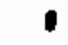 & 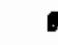 & & 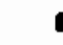 & 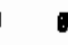 & 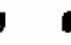 & 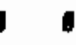 &. & 0 & 15 \\
\hline & & 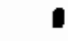 & 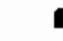 & & . & 0 & 1 & 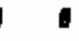 & \\
\hline
\end{tabular}

\begin{tabular}{|c|c|c|c|c|c|c|c|c|c|}
\hline 1086 & 1997 & 1988 & 1980 & 1990 & 1991 & 1992 & 1993 & 1994 & 1995 \\
\hline 22 & 22 & 80 & 102 & 122 & 146 & 180 & 269 & 229 & 272 \\
\hline 5 & 2 & 2 & 24 & 47 & 47 & 71 & 71 & 93 & 93 \\
\hline$\theta$ & 25 & 49 & 72 & 119 & 148 & 195 & 219 & 285 & 289 \\
\hline 0 & 15 & 16 & 46 & 45 & 89 & 80 & 109 & 139 & 139 \\
\hline 1 & 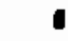 & 20 & $\mathbf{2 0}$ & 42 & 70 & 76 & 94 & 118 & 118 \\
\hline 6 & 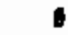 & 6 & $\theta$ & 3 & 23 & 23 & 44 & 61 & 81 \\
\hline 0 & 0 & ) & ฮ & 10 & 10 & 41 & 41 & 79 & 100 \\
\hline 1 & 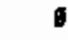 & $\theta$ & - & 4 & 25 & 95 & 129 & 162 & 233 \\
\hline 0 & $\theta$ & 1 & 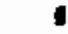 & 0 & 37 & 37 & 71 & 194 & 104 \\
\hline 0 & 0 & 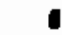 & $\oplus$ & 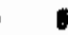 & I & 35 & 35 & 87 & 181 \\
\hline 0 & 0 & 6 & 6 & $\theta$ & g & 12 & 12 & 41 & 41 \\
\hline 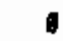 & 0 & 9 & 0 & 0 & 1 & 9 & 8 & 35 & 67 \\
\hline 0 & 1 & ๑ & 0 & 0 & 1 & 8 & 24 & 53 & 53 \\
\hline 9 & 1 & . & 0 & 0 & 0 & 0 & 6 & 21 & 46 \\
\hline 1 & 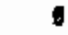 & 1 & $\theta$ & 1 & 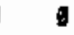 & 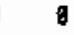 & 6 & 7 & 7 \\
\hline 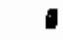 & 1 & $\theta$ & 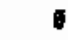 & ) & 1 & 0 & 6 & 2 & 27 \\
\hline 0 & 1 & 8 & 0 & $\theta$ & g & 8 & 1 & 6 & 7 \\
\hline & 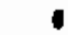 & 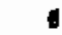 & 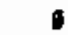 & 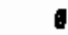 & ๑ & a & 6 & 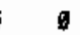 & 2 \\
\hline
\end{tabular}

PIR TOTN

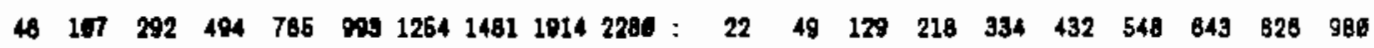

BUR TOTN

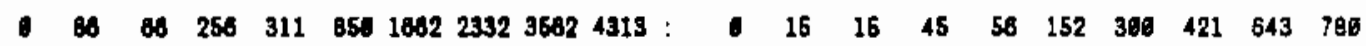

TOTN

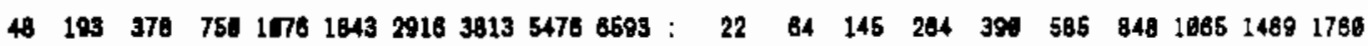


TABLE A.6. Upper Reference Case, Maximum At-Reactor Capacity--

Projected Cumulative Storage Requirements (contd)

NSSERLIES

UTIF

POOL

STRT 112

PALISADES

OCONEE 112

HILSTONE 1

ST LUCIE :

ROBINSON 2

GRNSTICX 1

LANUE CTY 112

PEACHEOTTOU 2

PERCHBOTTOU 3

PILGRIU 1

CALVERT CLF 112

gRNSTICK 2

OCOMEI 3

OYSTER CRK 1

PRAIRIE ISL 112

INOIAN PT 2

COOPER STY

ZION 112

Brina 142

B VNLFY 1

UIUSTONE 2

LIVGICK 1

POINT BENC 112

BRAIOTOOO 112

Bro RDCK 1

WAINE YANKEE

FORT CNHOUN

SERUOYAH $1 \mathrm{L2}$

DAVIS-BESSE I

LARROSSE

ENRICD FERI-2

MINE UILE PT:

SN ONOFRE $1,2,13$

MADDN MECX

CodK 122

FITZPATRZCK

SNLE 1

ARK MUCLEAR 1

ORESDEN 2

DUANE ARNOLO

WASH MUCEAR 2

PERRY 1:2

NORTH ANK 112

KGANNEE

ORESDEN 3

LIUERICX 2

ARK MUXLEAR 2

\begin{tabular}{|c|c|c|c|c|c|c|c|c|c|c|c|c|c|c|c|c|c|c|c|}
\hline 996 & 897 & 98 & 900 & 2894 & 201 & 2002 & 2013 & 2094 & 2905 & $\underline{1098}$ & 1997 & 1990 & $\underline{1909}$ & 2085 & $\underline{2901}$ & $\underline{2602}$ & $\underline{2003}$ & 2994 & 2805 \\
\hline$\overline{632}$ & 575 & 781 & 862 & 840 & 929 & 86 & $\overline{1014}$ & 181 & 137 & $\overline{201}$ & 311 & $\overline{340}$ & 388 & 388 & 420 & 443 & 465 & 595 & 522 \\
\hline 298 & 288 & 343 & 96 & 398 & 460 & 468 & 11 & 687 & 587 & 2 & 112 & 134 & 54 & 54 & 75 & 75 & 99 & 21. & 221 \\
\hline 715 & 1 & 011 & .16 & 1898 & 1144 & 184 & 1213 & 905 & 380 & 331 & 375 & 375 & 419 & 467 & 511 & 511 & 582 & 604 & 843 \\
\hline 931 & 931 & 1094 & 1904 & 1265 & 1425 & 1425 & 1095 & 16015 & 745 & 160 & 160 & 196 & 195 & 225 & 253 & 253 & 285 & 85 & 310 \\
\hline 368 & 9 & 29 & & 564 & 554 & 698 & 877 & & 739 & 141 & 163 & 163 & 188 & 11 & 11 & 31 & 258 & 58 & 278 \\
\hline 180 & 28 & 228 & 85 & 366 & 388 & 349 & 393 & 433 & 433 & $\pi$ & 97 & 97 & 113 & 131 & 31 & 148 & 167 & 185 & 185 \\
\hline 536 & 84 & 233 & 833 & 981 & $\% 1$ & 1122 & 1289 & 1289 & 418 & 109 & 120 & 150 & 158 & 585 & 85 & 210 & 241 & 41 & 265 \\
\hline 1442 & 1018 & 1972 & 2145 & 2333 & 2683 & 2839 & 3938 & $34 \pi 4$ & 3657 & 202 & 295 & 359 & 390 & 425 & 488 & 517 & 553 & 28 & 647 \\
\hline 755 & 938 & 938 & 1117 & 1312 & 2312 & 1473 & 1078 & 1678 & 1838 & 134 & 167 & 187 & 198 & 239 & 233 & 281 & 298 & 296 & 326 \\
\hline 504 & 738 & 913 & 913 & 1005 & 1267 & 1267 & 1461 & 1844 & 1040 & 101 & 131 & 182 & 182 & 195 & 25 & 225 & 288 & 191 & 291 \\
\hline 233 & 3987 & 387 & 538 & 539 & 695 & 895 & 888 & 658 & 1643 & 41 & 60 & 88 & 95 & 95 & 123 & 123 & 153 & $: 53$ & 177 \\
\hline 248 & 318 & 303 & 485 & 543 & 815 & 880 & 784 & 840 & 984 & 93 & 128 & 148 & 175 & 264 & 231 & 256 & 287 & 316 & 340 \\
\hline 422 & 422 & 572 & & 717 & 884 & 905 & 905 & 1149 & 1278 & 79 & 79 & 107 & 134 & 134 & 32 & 188 & 188 & 15 & 239 \\
\hline 90 & 147 & 198 & 243 & 243 & 291 & 332 & 332 & 392 & 424 & 46 & 68 & 91 & 113 & 113 & 134 & 154 & 154 & .77 & 196 \\
\hline 108 & 288 & 288 & 418 & 418 & 565 & 878 & 878 & 823 & 942 & 31 & 51 & 51 & 73 & 73 & 98 & 119 & 119 & 245 & 168 \\
\hline 105 & 169 & 233 & 298 & 364 & 398 & 452 & 525 & 591 & 647 & 37 & 64 & 83 & 165 & \pm 29 & 140 & 180 & 188 & 99 & 229 \\
\hline 15 & 60 & 69 & 123 & 181 & 181 & 229 & 229 & 288 & 334 & 7 & 31 & 31 & 58 & 82 & 82 & 163 & 103 & 129 & 151 \\
\hline$\%$ & 188 & 294 & 367 & 483 & 551 & 836 & 731 & 823 & 890 & 17 & 34 & 51 & 67 & 64 & 100 & 115 & 33 & 150 & 164 \\
\hline 32 & 90 & 148 & 261 & 322 & 379 & 481 & 547 & 697 & 798 & 15 & 41 & 88 & 119 & 147 & 173 & 220 & 256 & 277 & 324 \\
\hline 163 & 170 & 238 & 355 & 427 & 490 & 608 & 684 & 751 & 858 & 44 & 72 & 101 & 150 & 181 & 211 & 257 & 289 & 318 & 362 \\
\hline 25 & 84 & $\theta$ & 142 & 295 & 205 & 258 & 3 & 323 & 374 & 12 & 30 & 39 & 85 & 95 & 95 & 118 & 149 & 49 & 172 \\
\hline 77 & 86 & 144 & 144 & 286 & 204 & 284 & 331 & 390 & 391 & 11 & 35 & 59 & 50 & 84 & 107 & 167 & 134 & 159 & 159 \\
\hline 82 & 92 & 237 & 467 & 407 & 578 & 731 & 731 & 911 & 1681 & 11 & 11 & 42 & 72 & 72 & 102 & 135 & 130 & 162 & 188 \\
\hline 42 & 93 & 144 & 194 & & 299 & 344 & 491 & 454 & 480 & 15 & 33 & 51 & 89 & 88 & & 122 & 42 & 31 & 177 \\
\hline - & 5 & 141 & 141 & 292 & 437 & 437 & 559 & 693 & 893 & 1 & 2 & 69 & 60 & 124 & 185 & 105 & 236 & 293 & 293 \\
\hline 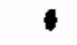 & 2 & 16 & 33 & 51 & 67 & 87 & B5 & 101 & 115 & ฮ & $\checkmark$ & 2 & 4 & 7 & 9 & 9 & 11 & 20 & 15 \\
\hline 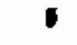 & 8 & 8 & 86 & 128 & 128 & 180 & 247 & 247 & 298 & 8 & 3 & 3 & 25 & 49 & 49 & 68 & 94 & 94 & 113 \\
\hline 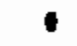 & 34 & 34 & 87 & 185 & 185 & 138 & 17 & $1 \pi$ & 267 & - & 12 & 12 & 24 & 38 & 38 & 49 & 34 & 64 & 75 \\
\hline$\bullet$ & 48 & 111 & 174 & 311 & 311 & 424 & 497 & 584 & 78 & 0 & 21 & 51 & 80 & 143 & 143 & 195 & 229 & 60 & 311 \\
\hline 1 & 47 & 47 & 94 & 148 & 148 & 180 & 243 & 243 & 285 & 1 & 22 & 22 & 44 & 88 & 68 & 88 & 114 & 124 & 133 \\
\hline 1 & 0 & 12 & 12 & 33 & 33 & 49 & 49 & 69 & 89 & • & - & 2 & 2 & $\mathbf{s}$ & 5 & B & 8 & $: 1$ & 11 \\
\hline$\bullet$ & - & 136 & 130 & $3 \pi$ & 377 & 583 & 842 & 842 & 1048 & - & $\bullet$ & 24 & 24 & 89 & 69 & 100 & 153 & 153 & 190 \\
\hline . & 1 & 128 & 128 & 285 & 285 & 418 & 410 & 589 & 569 & 0 & - & 22 & 22 & 49 & 49 & 71 & 71 & 98 & 98 \\
\hline - & $\theta$ & 85 & 129 & 249 & 320 & 420 & 501 & 819 & 682 & 6 & 1 & 26 & 54 & 102 & 133 & 173 & 298 & 256 & 283 \\
\hline a & 1 & 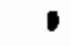 & 20 & 28 & 82 & 160 & 148 & 148 & 185 & 6 & - & 0 & 8 & 8 & 28 & 41 & 81 & 81 & 76 \\
\hline 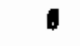 & 0 & 8 & 4 & 147 & 211 & 273 & 348 & 419 & 537 & 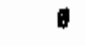 & 0 & 9 & 2 & 63 & 93 & 118 & 151 & 181 & 232 \\
\hline 1 & $\theta$ & 1 & 32 & 102 & 102 & 380 & 485 & 485 & 588 & 6 & - & $\theta$ & 8 & 32 & 32 & 54 & 83 & 83 & 164 \\
\hline 0 & 1 & b & 3 & 3 & 76 & 129 & 129 & 199 & 258 & 6 & 1 & $a$ & 1 & 1 & 32 & 59 & 59 & 91 & 118 \\
\hline - & 1 & 0 & 6 & 35 & 35 & 74 & 74 & 120 & 180 & $B$ & 6 & 0 & 6 & 18 & 16 & 34 & 34 & 56 & 74 \\
\hline 6 & $\theta$ & 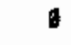 & - & $\theta$ & 133 & 133 & 275 & 407 & 407 & 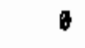 & 6 & - & 8 & 1 & 22 & 22 & 48 & 68 & SB \\
\hline 1 & $\bullet$ & 0 & $\theta$ & 34 & 34 & 129 & 220 & 228 & 317 & $\theta$ & 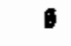 & 1 & t & 8 & 6 & 21 & 41 & 41 & 56 \\
\hline - & 0 & 6 & 1 & 133 & 281 & 374 & 519 & 853 & 784 & 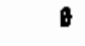 & 1 & 0 & 9 & 23 & 48 & 86 & 91 & 115 & I35 \\
\hline 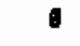 & B & 6 & 0 & 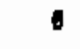 & 42 & 242 & $\pi 8$ & 941 & 1068 & 8 & 6 & - & $\theta$ & - & B & 44 & 142 & 171 & 194 \\
\hline g & $\theta$ & 0 & $\theta$ & 9 & 10 & 188 & 184 & 217 & 367 & 8 & 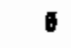 & 0 & 1 & 6 & 7 & 49 & 76 & 160 & 142 \\
\hline ๑ & 日 & 1 & 0 & 0 & 18 & 44 & 78 & 109 & 135 & 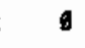 & 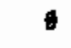 & g & 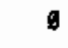 & 6 & 7 & 17 & 36 & 41 & 51 \\
\hline 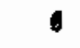 & 6 & 0 & - & g & 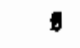 & 48 & 48 & 177 & 288 & . & 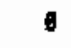 & 0 & 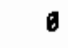 & $\theta$ & 9 & 8 & 8 & 36 & 48 \\
\hline & - & 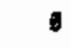 & 0 & 0 & 1 & 82 & 82 & 318 & 428 & 0 & ઇ & 6 & 6 & $\theta$ & 0 & 15 & 15 & 58 & 76 \\
\hline 1 & 0 & 0 & 6 & B & 0 & 0 & $46^{\circ}$ & 45 & 92 & 0 & 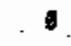 & 8 & 6 & $\theta$ & 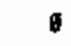 & $\theta$ & 19 & 19 & 38 \\
\hline
\end{tabular}


TABLE A.6. Upper Reference Case, Maximum At-Reactor Capacity--

Projected Cumulative Storage Requi rements (contd)

ASSE:IES

ITIN

POOL

CLINTON 1

OINNA

RAKCHO SECQ-1

BROTLS FERRY3

VT YANKEE 1

HATCH 12

SUSquetaNNA 1 L2

SNLE UNIT 2

ST LUCIE 2

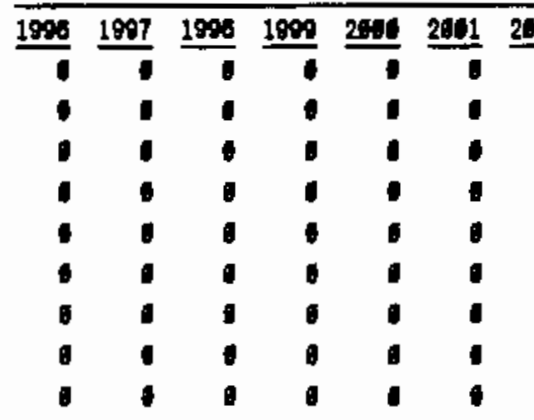

1900100710081000200020912042200320042985

PTR TOTN

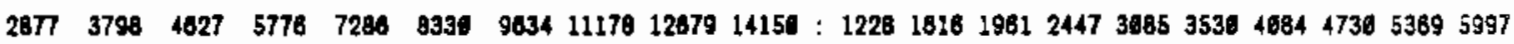

BIR TOTN

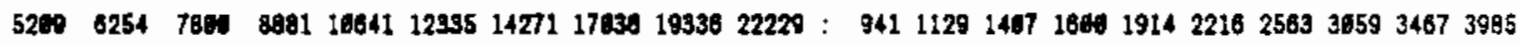

TOTAL

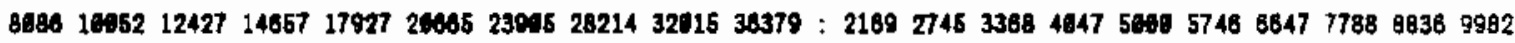


IABLE A.6. Upper Reference Case, Maximum At-Reactor Capacity--

Projected Cumulative Storage Requirements (contd)

ASSEtaLIES

VTIHU

POEL

SURRY 112

PALISADES

DCONEE 1L2

MIUSTONE 1

ST LUCIE

RDBINSON 2

BRUNSUICK I

LASNLLE CTY IL2

PEACHBOTTOU 2

PEACHEOTTOS 3

PILGRIU 1

CALVERT QF $1 \mathrm{H2}$

BRUNSIICK 2

OCONEE 3

OYSTER CRX 1

PRAIRIE ISC $1 \mathrm{L2}$

INDIN PT 2

COCPER STN

ZION 112

BYRON 112

Q VNLEY 1

UIUSTONE 2

LIMERICX 1

POINT QEACH 1 LO

BRAIDWOOO 142

BIG ROCX 1

WAINE YANKEE

FORT CALHOLY

SERUOYAH 1 HO

OAYIS-BESSE 1

LACROSSE

ENRICO FERI-2

NINE YILE PT1

SAN ONOFRE $1,2,13$

HADDAN NECX

COOX 112

FITZPATRICK

SALEI !

ARK NUCLEAR 1

DRESOEN 2

DSANE ARNOLD

WASH MUCLEAR 2

PERRY 142

NORTH ANNA 112

KETAUMEE

DRESDEN 3

LIMERICX 2

ARK NUCLEAR 2

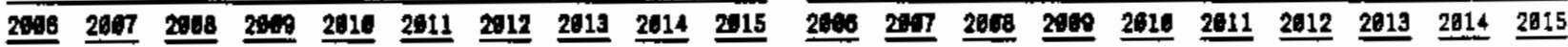

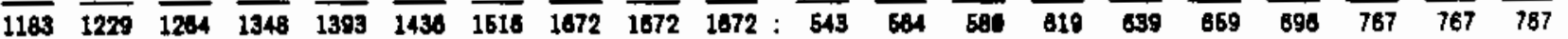
$\begin{array}{llllllllllllllllllll}626 & 620 & 673 & 732 & 732 & 789 & 789 & 789 & 789 & 789 & 244 & 244 & 282 & 285 & 285 & 397 & 307 & 3907 & 367 & 307\end{array}$

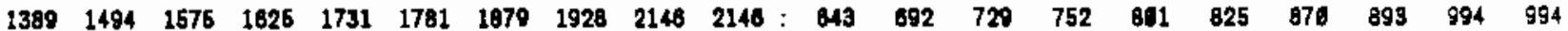

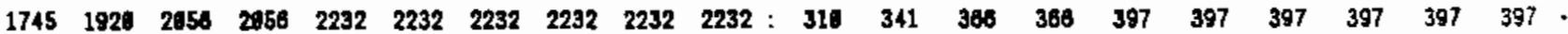
$\begin{array}{lllllllllllllllllllll}796 & 796 & 947 & 908 & 948 & 970 & 1031 & 1031 & 1084 & 1135 & : & 383 & 303 & 323 & 346 & 346 & 376 & 393 & 393 & 412 & 432\end{array}$ $\begin{array}{lllllllllllllllllllll}485 & 527 & 527 & 678 & 626 & 870 & 870 & 670 & 670 & 671 & : & 280 & 224 & 224 & 245 & 284 & 284 & 284 & 284 & 284 & 284\end{array}$ $\begin{array}{lllllllllllllllllllll}1579 & 1579 & 1795 & 1765 & 1796 & 1765 & 1705 & 1766 & 1796 & 1795 & 2 & 295 & 295 & 319 & 319 & 319 & 319 & 319 & 319 & 319 & 319\end{array}$

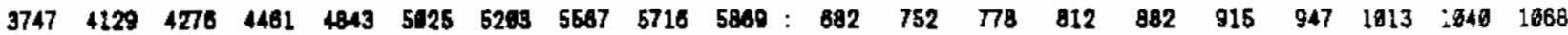

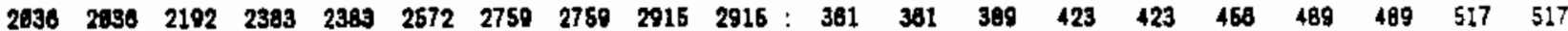

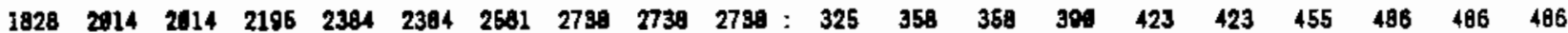

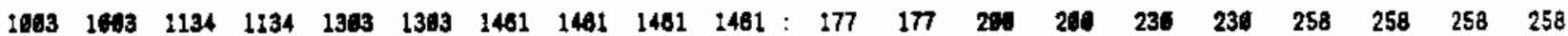

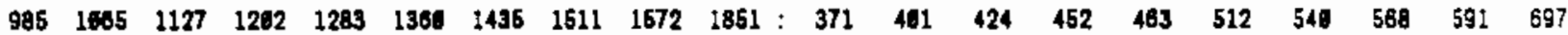
$\begin{array}{lllllllllllllllllllll}1278 & 1449 & 1580 & 1588 & 1727 & 1889 & 1896 & 2432 & 2158 & 2158 & 239 & 269 & 293 & 293 & 323 & 352 & 352 & 380 & 494 & 464\end{array}$

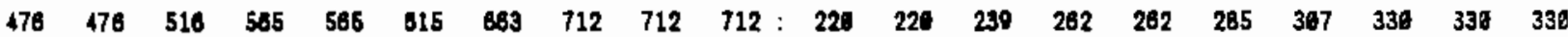
$\begin{array}{lllllllllllllllllllll}942 & 1096 & 1213 & 1213 & 1213 & 1213 & 1213 & 1213 & 1213 & 1213 & : & 166 & 194 & 214 & 214 & 214 & 214 & 214 & 214 & 214 & 214\end{array}$ $\begin{array}{llllllllllllllllllllll}889 & 763 & 897 & 872 & 943 & 1960 & 1975 & 1141 & 1289 & 1289 & 2 & 242 & 267 & 288 & 369 & 334 & 358 & 381 & 464 & 457 & 457\end{array}$

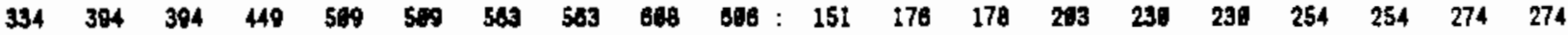

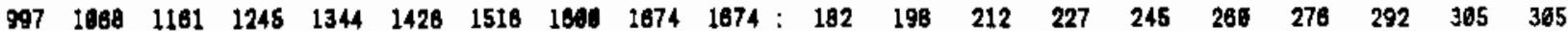

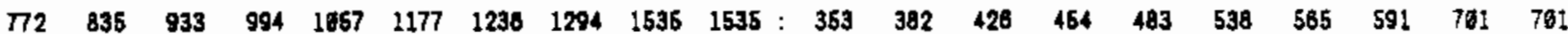

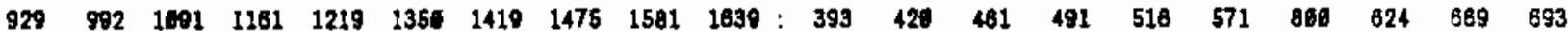

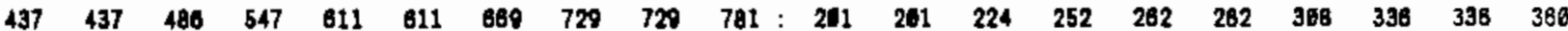
$\begin{array}{llllllllllllllllllll}454 & 517 & 517 & 576 & 648 & 641 & 698 & 757 & 757 & 897: & 185 & 216 & 218 & 234 & 268 & 268 & 284 & 388 & 380 & 328\end{array}$ $\begin{array}{lllllllllllllllllllll}1001 & 1248 & 1394 & 1394 & 1589 & 1759 & 1750 & 1936 & 2583 & 2983 & 169 & 221 & 247 & 247 & 280 & 312 & 312 & 343 & 369 & 369\end{array}$ $\begin{array}{lllllllllllllllllllll}558 & 611 & 855 & 705 & 762 & 996 & 933 & 933 & 933 & 939 & : & 197 & 217 & 233 & 258 & 271 & 322 & 331 & 331 & 331 & 391\end{array}$ $\begin{array}{llllllllllllllllllll}024 & 952 & 952 & 1679 & 1196 & 1195 & 1321 & 1433 & 1433 & 1538 & 348 & 493 & 463 & 456 & 505 & 505 & 559 & 606 & 606 & 658\end{array}$

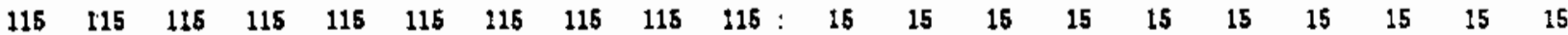

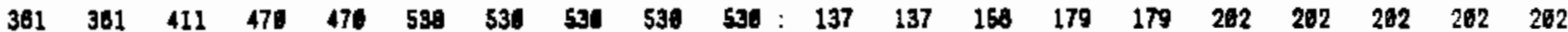

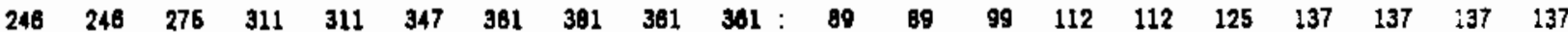

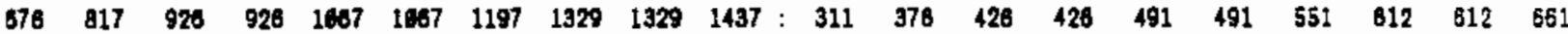
$\begin{array}{llllllllllllllllllll}338 & 338 & 379 & 429 & 429 & 479 & 529 & 529 & 571 & 612: & 158 & 158 & 177 & 201 & 201 & 224 & 248 & 248 & 267 & 288\end{array}$ $\begin{array}{llllllllllllllllllll}90 & 91 & 186 & 186 & 166 & 186 & 180 & 166 & 186 & 186 & 15 & 16 & 17 & 17 & 17 & 17 & 17 & 17 & 17 & 17\end{array}$ $\begin{array}{lllllllllllllllllllll}1046 & 1296 & 1298 & 1546 & 1797 & 1797 & 2035 & 2035 & 2232 & 2232 & : & 198 & 236 & 236 & 281 & 327 & 327 & 379 & 370 & 486 & 486\end{array}$ $\begin{array}{lllllllllllllllllllll}730 & 739 & 857 & 857 & 1016 & 1016 & 1016 & 1016 & 1016 & 1016 & : & 125 & 125 & 147 & 147 & 174 & 174 & 174 & 174 & 174 & 174\end{array}$ $\begin{array}{llllllllllllllllllll}883 & 883 & 1046 & 1271 & 1349 & 1424 & 1497 & 157 ! & 1033 & 1698 & 306 & 366 & 431 & 521 & 554 & 588 & 817 & 649 & 676 & 762\end{array}$ $\begin{array}{lllllllllllllllllllll}231 & 277 & 277 & 27 & 277 & 277 & 27 & 277 & 277 & 277 & : & 95 & 114 & 114 & 114 & 114 & 114 & 114 & 114 & 114 & 114\end{array}$ $\begin{array}{llllllllllllllllllll}866 & 882 & 787 & 884 & 941 & 1007 & 1679 & 1217 & 1271 & 1524 & 269 & 294 & 344 & 374 & 485 & 438 & 465 & 524 & 549 & 662\end{array}$ $\begin{array}{lllllllllllllllllllll}741 & 741 & 863 & 1090 & 1909 & 1155 & 1297 & 1297 & 1417 & 1539: & 132 & 132 & 153 & 179 & 179 & 285 & 230 & 230 & 252 & 273\end{array}$

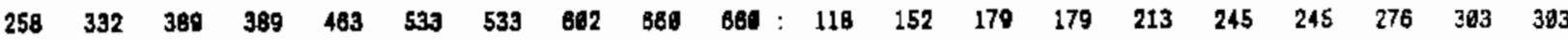

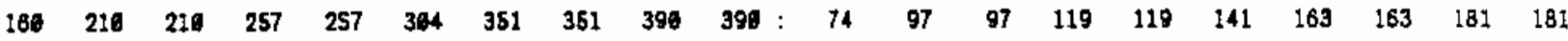
$\begin{array}{lllllllllllllllllllll}544 & 881 & 681 & 816 & 816 & 816 & 818 & 816 & 816 & 818 & : & 91 & 114 & 114 & 197 & 137 & 137 & 137 & 137 & 137 & 137\end{array}$

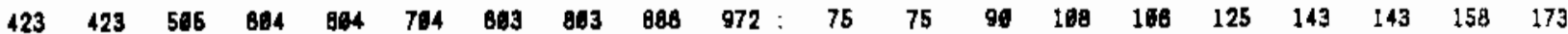
$\begin{array}{llllllllllllllllllll}993 & 1042 & 1151 & 1286 & 1427 & 1568 & 1801 & 1622 & 1931 & 2844 & 159 & 183 & 283 & 228 & 251 & 275 & 298 & 321 & 340 & 360\end{array}$

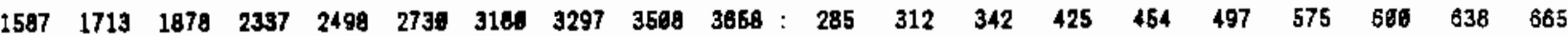
$\begin{array}{lllllllllllllllllllll}362 & 418 & 695 & 506 & 916 & 724 & 724 & 824 & 968 & 998 & 187 & 193 & 293 & 233 & 286 & 334 & 334 & 391 & 419 & 419\end{array}$ $\begin{array}{lllllllllllllllllllll}168 & 291 & 228 & 256 & 298 & 319 & 359 & 379 & 494 & 494: & 94 & 76 & 86 & 97 & 109 & 121 & 133 & 144 & 153 & 153\end{array}$ $\begin{array}{lllllllllllllllllllll}288 & 423 & 536 & 539 & 667 & 798 & 798 & 798 & 798 & 798 & : & 48 & 71 & 89 & 89 & 112 & 134 & 134 & 134 & 134 & 134\end{array}$

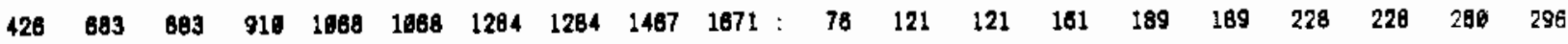
$\begin{array}{llllllllllllllllllll}92 & 151 & 198 & 198 & 257 & 257 & 312 & 389 & 369 & 414: & 38 & 83 & 82 & 82 & 107 & 107 & 136 & 153 & \div 53 & 172\end{array}$ 
TABLE A.6. Upper Reference Case, Maximum At-Reactor Capacity--

Projected Cumulative Storage Requirements (contd)

ASSEALIES

\begin{tabular}{|c|}
\hline POOL \\
\hline QINTON 1 \\
\hline GINMA \\
\hline RAKHO SECO-1 \\
\hline GROTISS FERRYG \\
\hline VT YANKEE 1 \\
\hline HАTCH 112 \\
\hline SUSQLEUANNA 112 \\
\hline SNLEI UNIT 2 \\
\hline ST LUCIE 2 \\
\hline PHO VERDE 1 \\
\hline PALO YERDE 2 \\
\hline TLRXEY PT 112 \\
\hline BROWIS FEPRY1L2 \\
\hline WATTS BAR 1L2 \\
\hline FAREY 1 \\
\hline CRTSTAL RYR 3 \\
\hline VOGTLE 112 \\
\hline TROJAN \\
\hline INDIN PT \\
\hline CNLATAY 1 \\
\hline RVR BEID 1 \\
\hline WATERFORD 3 \\
\hline MONTICELCO \\
\hline PALO VERDE 3 \\
\hline QUND CITIES $1 L 2$ \\
\hline OIAQD CANYON-1 \\
\hline WNP-3 \\
\hline DIARLD CAMYON-2 \\
\hline FARLEY 2 \\
\hline IXEJIRE 1 \\
\hline HOPE CREEX \\
\hline MOLF CREE 1 \\
\hline SEABROOK 1 \\
\hline SLATER 1 \\
\hline CQUANCHE PK 112 \\
\hline ICOUIRE 2 \\
\hline SHOREHAN \\
\hline
\end{tabular}

\begin{tabular}{|c|c|c|c|c|c|c|c|c|c|c|c|c|c|c|c|c|c|c|c|}
\hline 2906 & $\theta 7$ & 2090 & 290 & 2010 & 2011 & $\underline{2012}$ & 2113 & 2014 & 2015 & 2906 & 2007 & 2648 & 2009 & 2610 & 2911 & 2912 & $\underline{2013}$ & 2014 & $\underline{2915}$ \\
\hline 379 & $5 n$ & $\mathrm{C}_{2}$ & 677 & $\overline{815}$ & 815 & 987 & 1888 & 1880 & 1216 & 89 & 92 & 92 & 124 & 149 & 148 & 17 & 198 & 198 & 222 \\
\hline 1113 & 131 & 163 & 170 & 277 & 207 & $2 \pi 7$ & 27 & 27 & 27 & 34 & 49 & 50 & 50 & 68 & 68 & 68 & 88 & 68 & 88 \\
\hline $1 / 2$ & 160 & 156 & 27 & 257 & 257 & $3 \pi 7$ & 360 & 368 & $3 \%$ & 47 & 72 & 72 & 96 & 119 & 119 & 142 & 185 & 165 & 184 \\
\hline 408 & 489 & 504 & $\mathbf{S 4}$ & 765 & 955 & 955 & 1143 & 1143 & 1311 & 74 & 74 & 102 & $1: 2$ & 139 & 173 & 173 & 208 & 298 & 236 \\
\hline 274 & 274 & 361 & 189 & 469 & 575 & 881 & 001 & 081 & 021 & 50 & 50 & 68 & 86 & 86 & 186 & 125 & 125 & 125 & 125 \\
\hline 445 & 624 & 854 & $10 \mathrm{sec}$ & 1100 & 1470 & 1820 & 1772 & 2592 & 2582 & B3 & $\$ 12$ & 159 & 187 & 218 & 273 & 301 & 329 & 479 & 479 \\
\hline 539 & 734 & 142 & 1233 & 1428 & 1890 & 1838 & 2171 & $24 \pi 7$ & 2635 & 93 & 127 & 189 & 213 & 247 & 311 & 343 & 375 & 428 & 455 \\
\hline 141 & 214 & 214 & 285 & 350 & 359 & 427 & 400 & 400 & 554 & 85 & 98 & 98 & 131 & 105 & 185 & 198 & 229 & 229 & 256 \\
\hline 49 & 112 & 184 & 165 & 223 & 283 & 283 & 342 & 391 & 391 & 19 & 44 & 62 & 62 & 87 & 110 & 110 & 133 & 152 & 152 \\
\hline 41 & 97 & 141 & 194 & 250 & 304 & 357 & 411 & 456 & 501 & 20 & 42 & 61 & 85 & 109 & 133 & 158 & 179 & 199 & 219 \\
\hline 44 & 82 & 123 & 178 & 221 & 287 & 315 & 351 & 391 & 433 & 19 & 38 & 54 & 78 & 98 & 116 & 137 & 153 & 171 & 189 \\
\hline 85 & 107 & 146 & 210 & 251 & 269 & 359 & 564 & 654 & 564 & 30 & 49 & 84 & 96 & 115 & 133 & 165 & 254 & 254 & 254 \\
\hline $1 \boldsymbol{N}$ & 100 & 426 & 849 & 893 & 1182 & 1182 & 1558 & 1870 & 2834 & 29 & 20 & 76 & 148 & 146 & 215 & 215 & 283 & 340 & 478 \\
\hline $\boldsymbol{\varphi}$ & 180 & 200 & 347 & 490 & 458 & 517 & 582 & 84 & 713 & 46 & 74 & 96 & 160 & 185 & 211 & 239 & 259 & 396 & 329 \\
\hline - & 4 & 4 & $\Delta$ & 125 & 126 & 183 & 243 & 243 & 201 & $g$ & 2 & 2 & 30 & 58 & 58 & 84 & 112 & 112 & 134 \\
\hline 1 & 3 & 3 & 63 & 63 & 122 & 122 & 182 & 182 & 239 & 1 & $I$ & 1 & 29 & 29 & 57 & 57 & 84 & 84 & 107 \\
\hline 1 & 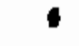 & 101 & 1.1 & 217 & 335 & 335 & 440 & 562 & 552 & $\theta$ & 1 & 47 & 47 & 160 & 155 & 155 & 97 & 255 & 255 \\
\hline 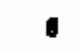 & $\bullet$ & 3 & 70 & 112 & 152 & 192 & 232 & 265 & 200 & 6 & g & 14 & 32 & 52 & 70 & 99 & 187 & 122 & 138 \\
\hline 1 & 6 & 35 & 35 & 102 & 102 & 163 & 225 & 225 & 277 & 6 & 1 & 18 & 16 & 47 & 47 & 74 & 163 & 163 & 126 \\
\hline 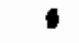 & 1 & 30 & 38 & 122 & 105 & 195 & 275 & 395 & 395 & - & $\theta$ & 16 & 10 & 52 & 82 & 82 & 118 & 142 & \pm 42 \\
\hline 4 & ป & 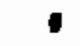 & 131 & 131 & 3144 & 475 & 475 & 617 & 703 & 1 & 6 & 1 & 24 & 24 & 58 & 88 & 88 & 114 & 141 \\
\hline 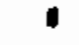 & $\bullet$ & • & 31 & 112 & 112 & 185 & 270 & 270 & 344 & 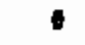 & $\theta$ & c & 13 & 47 & & 81 & $\$ 12$ & 112 & 141 \\
\hline 1 & 1 & 1 & 44 & 44 & 196 & 195 & 135 & 135 & 135 & - & 0 & 6 & 8 & 8 & 23 & 23 & 23 & 23 & 23 \\
\hline 6 & 1 & 1 & 1 & 34 & 82 & 131 & 175 & 214 & 255 & 8 & 1 & 5 & - & 15 & 36 & 57 & 76 & 93 & 111 \\
\hline 1 & 0 & $\bullet$ & 6 & 248 & 392 & 516 & 780 & 1492 & 1402 & - & 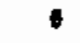 & a & 1 & 44 & 67 & 91 & 137 & 283 & 263 \\
\hline 6 & 1 & 5 & . & 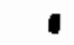 & 67 & 87 & 152 & 162 & 228 & . & 0 & $g$ & 6 & 1 & 31 & 31 & 76 & 70 & 105 \\
\hline 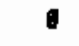 & 5 & 0 & 1 & 1 & 9 & 78 & 128 & 182 & 244 & 1 & 1 & 1 & 6 & 0 & 4 & 33 & 58 & 79 & 106 \\
\hline 1 & . & 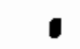 & 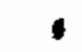 & 6 & 0 & 24 & 24 & 94 & 94 & 0 & 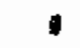 & 1 & 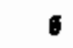 & 0 & 8 & 11 & 11 & 43 & 43 \\
\hline 1 & 0 & 1 & 6 & 1 & . & 1 & 24 & 71 & 71 & 0 & 9 & 6 & 0 & 6 & 8 & 0 & 11 & 33 & 33 \\
\hline 6 & 1 & 6 & 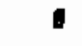 & - & 1 & 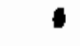 & 46 & 89 & 133 & 1 & $B$ & 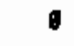 & 0 & 6 & 0 & 0 & 19 & 38 & 56 \\
\hline 1 & 5 & - & . & 1 & $\bullet$ & - & 117 & 272 & 272 & 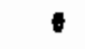 & 6 & 1 & $\theta$ & 0 & 1 & 0 & 21 & 49 & 49 \\
\hline - & 1 & 1 & ฮ & 1 & 0 & 1 & 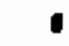 & 2 & 36 & 6 & 0 & 0 & 0 & 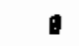 & 9 & 0 & 0 & 1 & 17 \\
\hline 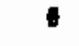 & 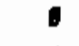 & 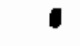 & 6 & • & • & 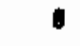 & - & 2 & 2 & 6 & 0 & 6 & 6 & 0 & (1) & 6 & 0 & 1 & 1 \\
\hline 1 & - & 1 & 5 & 1 & 1 & 0 & 6 & 31 & 79 & 0 & $\theta$ & 0 & 0 & g & 8 & g & 0 & 14 & 37 \\
\hline 6 & 6 & 5 & 6 & - & 1 & $\bullet$ & 6 & 36 & $12:$ & a & $\theta$ & 4 & 0 & 6 & 0 & 0 & $\theta$ & 15 & 48 \\
\hline$\theta$ & 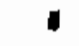 & - & 1 & $\boldsymbol{1}$ & 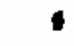 & 0 & o & 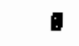 & 32 & 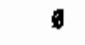 & 6 & g & 1 & 6 & 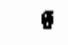 & $\theta$ & 9 & 0 & 14 \\
\hline a & 1 & a & 1 & $\theta$ & 1 & 0 & 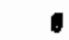 & ๑ & 62 & 6 & 6 & $\nabla$ & 0 & 0 & $\theta$ & $\theta$ & 0 & 0 & 11 \\
\hline
\end{tabular}

PUR TOTN

I5964 17868 $195832165823958260132811139702 \quad 3269034784: 6780 \quad 7503 \quad 8282 \quad 9198 \quad 16192 \quad 11973 \quad 1198413127 \quad 13996 \quad 14865$

BTR TOTNL

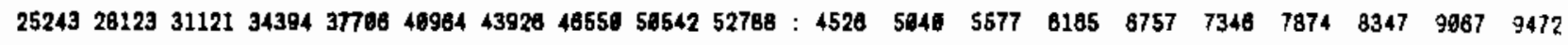

TOTAL

$41197458995962456450618586899772937772529323297492: 1128512543 \quad 1385915363169491841819858214742366324337$ 
TABLE A.6. Upper Reference Case, Maximum At-Reactor Capacity-Projected Cumulative Storage Requirements (contd)

\begin{tabular}{|c|c|c|c|c|c|c|c|c|c|c|}
\hline \multirow[b]{2}{*}{ POOL } & \multicolumn{5}{|c|}{ NSEDLIES } & \multicolumn{5}{|c|}{ MIIN } \\
\hline & $\underline{2016}$ & 2017 & 2018 & 2019 & 2424 & $\underline{2016}$ & 2017 & 2118 & 2019 & 2026 \\
\hline Uat 10 & $\overline{1672}$ & $\overline{1672}$ & $\overline{1672}$ & $\overline{1672}$ & $\overline{1672}$ & $\overline{767}$ & $\overline{767}$ & 787 & $\overline{767}$ & $\overline{767}$ \\
\hline PALISADES & 789 & 789 & 789 & 780 & 789 & 367 & 307 & 367 & 397 & 307 \\
\hline OCONEI 14 & 2148 & 2148 & 2146 & 2146 & 2148 & m & $\$ 4$ & $\infty$ & 94 & $\$ 4$ \\
\hline UIUSTONE 1 & 2232 & 2232 & 2232 & 2232 & 2232 & 397 & 397 & 397 & 397 & 397 \\
\hline ST LUCIE 1 & 1133 & 1139 & 1133 & 1133 & 1133 & 432 & 432 & 432 & 432 & 432 \\
\hline RogiKson 2 & 870 & 878 & 876 & 870 & 675 & 284 & 284 & 284 & 284 & 284 \\
\hline BPanstick 1 & 1705 & 1765 & 1705 & 1795 & 1795 & 319 & 319 & 319 & 319 & 319 \\
\hline LSALE TTY 14 & 8184 & 8357 & 6543 & 8828 & 7804 & 1126 & 1157 & 1191 & 1242 & 1274 \\
\hline PENCHEOTTON 2 & 2916 & 2015 & 2015 & 2015 & 2015 & 517 & 517 & 517 & 517 & 517 \\
\hline PENCHEOTTOU 3 & 2730 & 2738 & 2738 & 2738 & 2730 & 488 & 488 & 488 & 486 & 486 \\
\hline PILeRIU 1 & 1481 & 1461 & 1461 & 1481 & 1481 & 258 & 258 & 258 & 258 & 258 \\
\hline CALYET CLF 122 & $185 i$ & 1923 & 1923 & 1923 & 1923 & 697 & 724 & 724 & 724 & 724 \\
\hline BRASIICK 2 & 2158 & 2158 & 2158 & 2158 & 2168 & 44 & $4: 4$ & 464 & 494 & 494 \\
\hline oconer 3 & 712 & 712 & 712 & 712 & 712 & 331 & 330 & 330 & 330 & 33 \\
\hline OYSTER CRK 1 & 1213 & 1213 & 1213 & 1213 & 1213 & 214 & 214 & 214 & 214 & 214 \\
\hline RAIRIE ISL 112 & 1260 & 1289 & 1280 & 1289 & 1269 & 457 & 467 & 457 & 457 & 457 \\
\hline INDIN PT 2 & 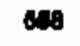 & 698 & 608 & 898 & 688 & 274 & 74 & 274 & 274 & 274 \\
\hline COOPER STM & 1874 & 1874 & 1874 & 1674 & 2674 & 3115 & 395 & 385 & 395 & 395 \\
\hline ION 112 & 1535 & 1536 & 1535 & 1535 & 1535 & 791 & 701 & $7 \oplus 1$ & 701 & 701 \\
\hline Bron 112 & 1891 & 1815 & 1886 & 1934 & 2058 & 715 & 768 & 798 & 818 & 870 \\
\hline VNLF 1 & 834 & 834 & 234 & 834 & 834 & 384 & 384 & 384 & 394 & 384 \\
\hline ПUSTONE 2 & 807 & 619 & 807 & 8.7 & 807 & 328 & 328 & 328 & 328 & 328 \\
\hline LIERICX 1 & 2237 & 2480 & 2496 & 2548 & 2718 & 397 & 427 & 427 & 451 & 182 \\
\hline POINT BEACH 14 & 933 & 933 & 933 & 933 & 933 & 331 & 331 & 331 & 331 & 331 \\
\hline BQAIDTHOD 112 & 1845 & 1640 & 1784 & 1851 & 1851 & 894 & 694 & 746 & 783 & 783 \\
\hline BIG RDCK 1 & 115 & 116 & 115 & 115 & 115 & 15 & 15 & 15 & 15 & 15 \\
\hline AINE YAUKEI & 5394 & 530 & 530 & 536 & 530 & 282 & 202 & 262 & 292 & 202 \\
\hline FORT CHLOUN & 391 & 381 & 381 & 381 & 381 & 137 & 137 & 137 & 137 & 37 \\
\hline SERLYYAH 112 & 1494 & 1558 & 1688 & 1888 & 1818 & 888 & 716 & $m$ & 777 & 838 \\
\hline DAVIS-BESSE 1 & 812 & 659 & 710 & 710 & 710 & 288 & 398 & 332 & 392 & 332 \\
\hline LACROSSE & 180 & 10 & 100 & 100 & 100 & 17 & 17 & 17 & 17 & 17 \\
\hline ENRICO FETI-2 & 2444 & 2673 & 2673 & 2863 & 2883 & 445 & 488 & 486 & 521 & 521 \\
\hline NINE MILE PT1 & 1018 & 1016 & 1018 & 1018 & 1818 & 174 & 174 & 174 & 174 & 174 \\
\hline SAN ONOFRE $1,2,13$ & 1704 & 1092 & 1902 & 2017 & 2917 & 736 & 79 & 796 & 84a & 040 \\
\hline HDONY MECX & 277 & 277 & $2 n$ & 277 & $2 n$ & 114 & 114 & 114 & 114 & 114 \\
\hline $\operatorname{coO} 112$ & 1524 & 1593 & 1867 & 1887 & 1867 & $\$ 82$ & 890 & 721 & 720 & 720 \\
\hline FITZPATRICK & 1630 & 1639 & 1539 & 1539 & 1539 & 273 & 273 & 273 & 273 & 273 \\
\hline SNEN 1 & 720 & 786 & 786 & 786 & 780 & 331 & 381 & 381 & 381 & 381 \\
\hline ARK MXLEAR 1 & 380 & 304 & 390 & 390 & 390 & 181 & 181 & 181 & 181 & $18 !$ \\
\hline DRESDEN 2 & 818 & 816 & 816 & 816 & 818 & 137 & 137 & 137 & 137 & 137 \\
\hline DUNE ARNQLD & 972 & 972 & 972 & 972 & 972 & 173 & 173 & 173 & 173 & 173 \\
\hline IASH MCAEAR 2 & 2158 & 2284 & 2419 & 2522 & 2850 & 380 & 492 & 428 & 444 & 487 \\
\hline PERRT 112 & 3976 & 4272 & 4458 & 4881 & 5970 & 795 & 777 & 011 & 848 & 922 \\
\hline NORTH ANA 112 & 990 & 1492 & 1248 & 1297 & 1338 & 480 & $5: 5$ & $5 \pi$ & 595 & 618 \\
\hline KENNNE & 4144 & $4 a$ & 464 & 464 & 404 & 163 & 153 & 153 & 153 & 153 \\
\hline DRESDEI 3 & 798 & 798 & 798 & 798 & 798 & 134 & 134 & 134 & 134 & 134 \\
\hline LIMERICX 2 & 1871 & 1889 & 1869 & 2048 & 2289 & 290 & 331 & 331 & 363 & 462 \\
\hline ARK MUCLEAR 2 & 414 & 469 & 526 & 528 & 581 & 172 & 195 & 218 & 218 & $24 !$ \\
\hline
\end{tabular}


TABLE A.6. Upper Reference Case, Maximum At-Reactor Capacity-Projected Cumulative Storage Requirements (contd)

\begin{tabular}{|c|c|c|c|c|c|c|c|c|c|c|}
\hline \multirow[b]{2}{*}{ POLL } & \multicolumn{5}{|c|}{ ASSEQIES } & \multicolumn{5}{|c|}{ STIF- } \\
\hline & 2015 & 2017 & 2018 & 2010 & $\underline{2021}$ & 216 & $\underline{2117}$ & $\underline{2610}$ & 2019 & 2120 \\
\hline CIINTO: 1 & 1353 & $\overline{1368}$ & $\overline{1517}$ & 1853 & 1868 & 248 & 248 & 277 & 362 & 302 \\
\hline GINM & 27 & $m$ & $\mathbf{2 \pi}$ & 27 & $2 \pi 7$ & 68 & 80 & 68 & 68 & 68 \\
\hline PUCHO SECO-1 & 300 & 300 & 390 & 390 & 390 & 184 & 184 & 184 & 184 & 184 \\
\hline BRWIS FERRY3 & 1485 & 1495 & 1495 & 1465 & 1465 & 200 & 208 & 280 & 208 & 286 \\
\hline VT YANKEE 1 & 881 & 801 & 601 & 681 & $\mathbf{8 8 1}$ & 125 & 125 & 125 & 125 & 125 \\
\hline MATCH $1 \mathbf{2}$ & 2714 & 2850 & 2860 & 2979 & 2979 & 504 & 631 & 631 & 553 & 563 \\
\hline SUSquENAM 142 & 2796 & 3150 & 3341 & 3406 & 3847 & 483 & 544 & 577 & 852 & 685 \\
\hline SNLA UNIT 2 & 814 & 614 & 685 & 749 & 740 & 283 & 283 & 310 & 341 & 341 \\
\hline ST WVIE 2 & 442 & 498 & 498 & 544 & 601 & 172 & 184 & 194 & 212 & 234 \\
\hline PNL VERDE 1 & 848 & 590 & 853 & 694 & 745 & 230 & 281 & 285 & $30 \mathrm{~s}$ & 325 \\
\hline PALO VERDE 2 & $4 n$ & 524 & 501 & 892 & 847 & 280 & 228 & 245 & 263 & 282 \\
\hline TURKEY PT 112 & 564 & 554 & 564 & 534 & 554 & 254 & 254 & 264 & 254 & 254 \\
\hline BRours FaRT1L & 2024 & 2024 & 2034 & 2034 & 2034 & 478 & 478 & 478 & 478 & 478 \\
\hline TATTS BAR :12 & 788 & 815 & ssi & 863 & 1000 & 353 & 376 & 307 & 444 & 408 \\
\hline FAREY 1 & 303 & 343 & 343 & $\mathbf{3 4 3}$ & $\mathbf{3 4 3}$ & 168 & 158 & 168 & 168 & 168 \\
\hline CISTAL RVR 3 & 230 & 205 & 285 & 285 & 285 & 107 & 132 & 132 & 132 & 132 \\
\hline VOGTE 142 & 657 & 782 & 782 & 871 & 980 & 393 & 361 & 361 & $4 \pi 2$ & 458 \\
\hline TROJAN & 334 & 394 & 334 & 334 & 334 & 154 & 154 & 164 & 154 & 154 \\
\hline INDIAN PT 3 & $2 \pi$ & 277 & $2 \pi$ & 277 & 277 & 126 & 128 & 128 & 128 & 120 \\
\hline CHLATAY I & 402 & 471 & 471 & 531 & 662 & 176 & 100 & 100 & 225 & 255 \\
\hline RVR RED 1 & 763 & O26 & 1104 & $11 \%$ & 1271 & 141 & 171 & 214 & 284 & 235 \\
\hline WATEFOPO 3 & 495 & 405 & 491 & 669 & 668 & 108 & 168 & 204 & 220 & 228 \\
\hline DonTrCa & 135 & 136 & 135 & 135 & 135 & 23 & 23 & 23 & 23 & 23 \\
\hline PNL VERDE 3 & 295 & 340 & 388 & 423 & 468 & 129 & 148 & 189 & 185 & 294 \\
\hline QUND CITIES 112 & 1492 & 1492 & 1492 & 1492 & 1492 & 203 & 263 & 203 & 263 & 263 \\
\hline DIALO CAMYNON-1 & 228 & 300 & 300 & 380 & 38 & 195 & 142 & 142 & 174 & 174 \\
\hline MPP-3 & 305 & 303 & 421 & 475 & 54 & 132 & 168 & IBA & 207 & 237 \\
\hline OIABL CAMYN-2 & 171 & 171 & 260 & 266 & 347 & 78 & $7 B$ & 119 & 119 & 150 \\
\hline FAREY 2 & 123 & 177 & 177 & 223 & 278 & 57 & 82 & 82 & 163 & 128 \\
\hline WCETPE 1 & 133 & 183 & 230 & $2 \pi$ & $2 \pi$ & 68 & 77 & 104 & 117 & 117 \\
\hline HDPE CREFX & 436 & $\$ 10$ & 819 & 768 & 945 & 79 & 113 & 113 & 138 & 172 \\
\hline TOLF CREXX 1 & 70 & 180 & 148 & 179 & 217 & 32 & 50 & 69 & 83 & 101 \\
\hline SEABRDax 1 & 43 & 80 & 137 & 171 & 217 & 20 & 41 & 83 & 79 & 100 \\
\hline SUngR: & 79 & 132 & 188 & 188 & 242 & 37 & 61 & 87 & 87 & 112 \\
\hline COUACHE PK 112 & 203 & 298 & 387 & 483 & 658 & 82 & 120 & 158 & 187 & 224 \\
\hline MCUIRE 2 & 78 & 128 & 128 & 169 & 228 & 33 & 54 & 54 & 71 & 93 \\
\hline SHOREYAM & 62 & 168 & 362 & 382 & 434 & 11 & 31 & 55 & 55 & 79 \\
\hline CRAND GJF 1 & 97 & 384 & 304 & 476 & 688 & 17 & 54 & 54 & 84 & 121 \\
\hline 日 VNLEY 2 & 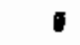 & 14 & 14 & 62 & 194 & 0 & 6 & 6 & 24 & 48 \\
\hline
\end{tabular}

\footnotetext{
PTR TOTAL $\quad 36193 \quad 37963 \quad 39494 \quad 46851 \quad 41989: 15522 \quad 1629818939 \quad 1747818686$

BIR TOTNL $\quad 54861571195827 B \quad 8968902252 ： 981010258164861878611173$

TOTN

$988549517397882109746 \quad 164249: 25332 \quad 285462739629282 \quad 29239$
} 
TABLE A.7. Upper Reference Case, Maximum At-Reactor Capacity Plus

Transshipment--Projected Annual Storage Requirements

NSSamiES

NTIH

POOL

19801997 1988 1989199119911992199319941005

1986199719881989 1990 $199119921993 \quad 19941995$

PALISADES

UILESTONE 1

PILGRIY !

CALYET aF 112

OYSTER CRK 1

PRAIRIE ISL IL2

NORTH ANNA 142

SURRY 112

INOTAN PT 2

COOPER STN

LIUERICX 1

LIMERICK 2

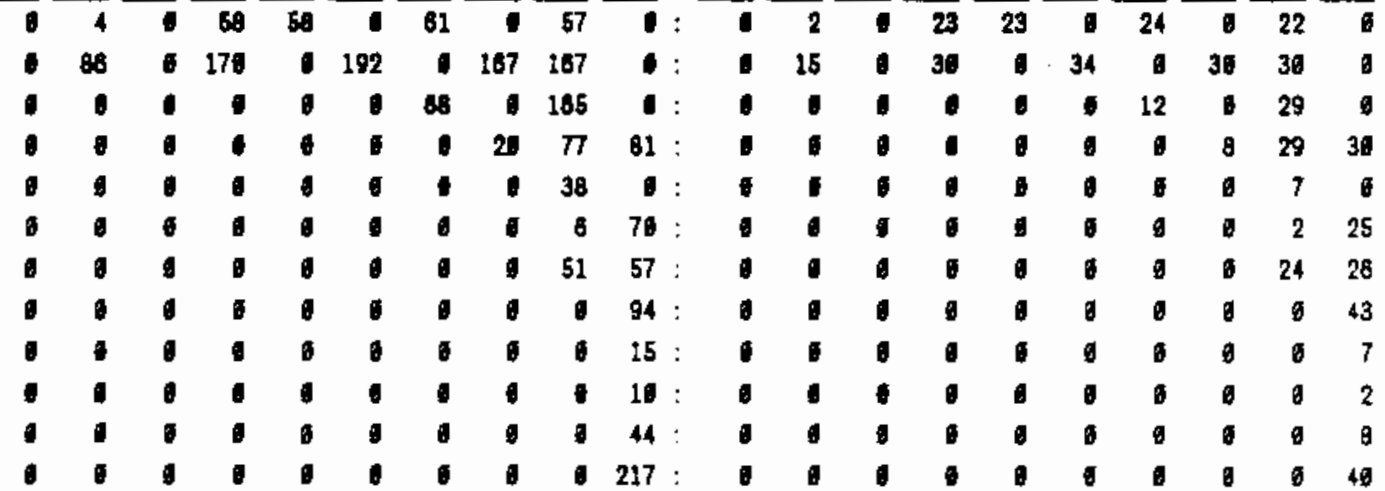

PIR TOTN

6

58 s9

$6120 \quad 191 \quad 317:$

$\begin{array}{lllllllll}-2 & 2 & 23 & 23 & 24 & 8 & 77 & 132\end{array}$

BUR TOTA

\begin{tabular}{lllllllll}
\hline & $86 \quad 170 \quad 192 \quad 68$ & 187 & 376 & 271
\end{tabular}

\begin{tabular}{llllllllll}
\hline & 15 & 3 & 39 & 34 & 12 & 36 & 86 & 49
\end{tabular}

TOTN

\begin{abstract}
19
\end{abstract}

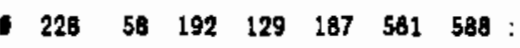

$\begin{array}{lllllllll}\text { - } & 17 & 53 & 23 & 34 & 36 & 37 & 142 & 181\end{array}$ 
TABLE A.7. Upper Reference Case, Maximum At-Reactor Capacity Plus Transshipment--Projected Annual Storage Requirements (contd)

ASSEIELIES

ITIN

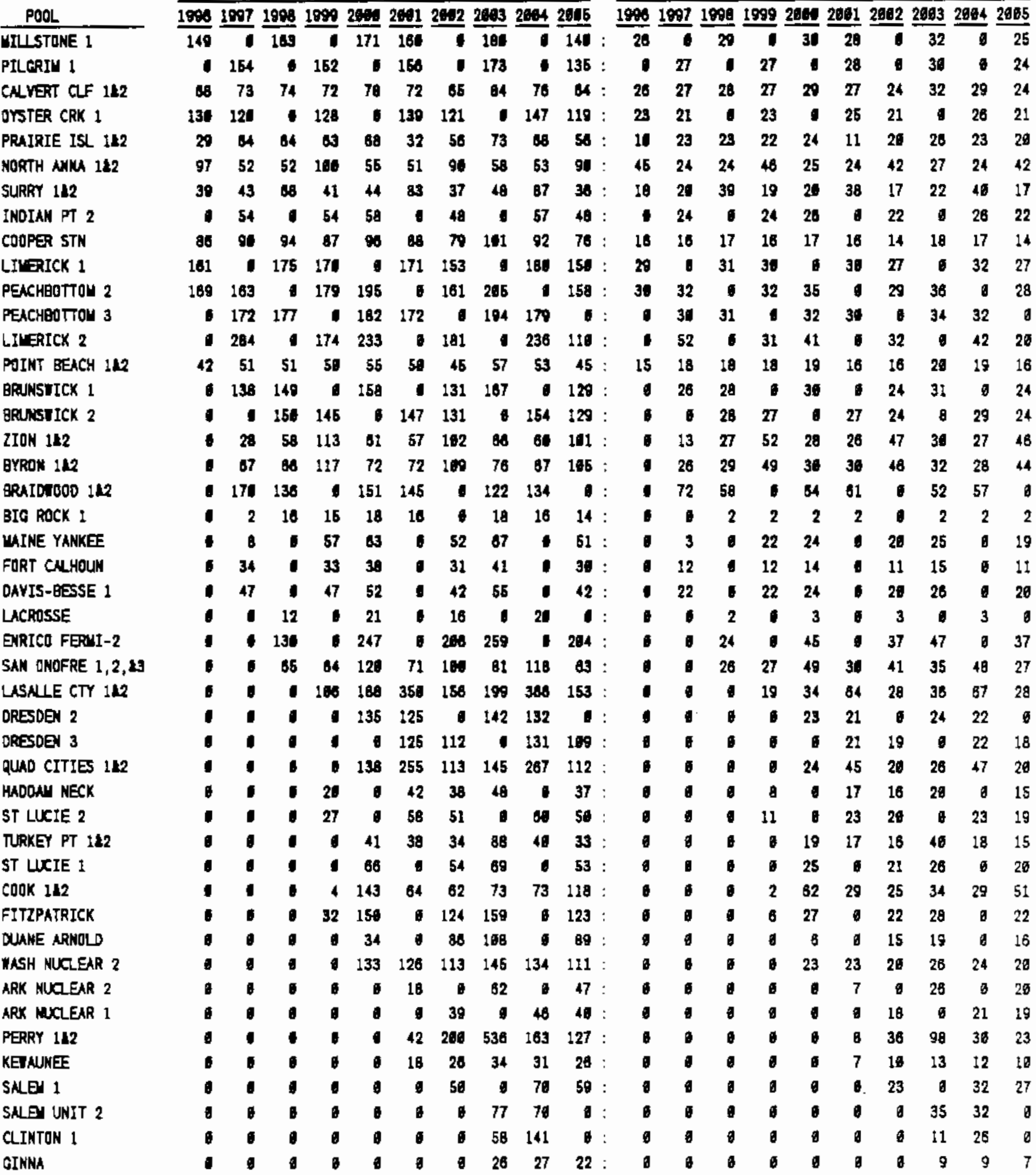




\section{IABLE A.7. Upper Reference Case, Maximum At-Reactor Capacity PTus Transshipment--Projected Annual Storage Requirements (contd)}

ASSER:IES

PQd RUWOHO SECO-1 VT YANKEE 1 HATCH 1 L2 suspeenarm 12 Q YNLEY 2

PRR TOTML

BNR TOTN

TOTN.

\begin{tabular}{|c|c|c|c|c|c|c|c|c|c|}
\hline 190 & 1907 & 1008 & 1000 & 2001 & 2091 & 2092 & 2013 & 2084 & 20015 \\
\hline 1 & & & 1 & 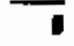 & 7 & $\overline{7}$ & 1 & 5 & 6 \\
\hline 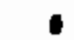 & 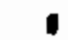 & ॥ & $\bullet$ & b & 】 & 1 & 74 & 0 & 80 \\
\hline 1 & 0 & ॥ & $\bullet$ & 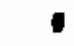 & 1 & . & 1 & 20 & 257 \\
\hline - & 1 & t & • & 1 & 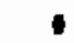 & 6 & 0 & 34 & 312 \\
\hline 1 & 6 & . & 1 & 1 & 1 & ๑ & $\bullet$ & 6 & 1 \\
\hline
\end{tabular}

VTIHM

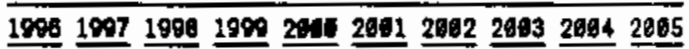

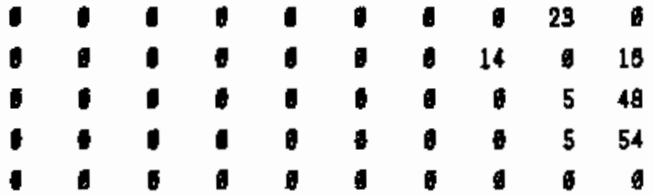

$\begin{array}{lllllllllllllllllllll}275 & 891 & 854 & 882 & 1185 & 87 ! & 1131 & 1396 & 1239 & 1217 & 113 & 287 & 271 & 361 & 483 & 367 & 472 & 544 & 522 & 511\end{array}$

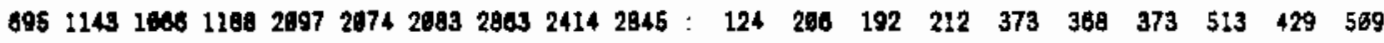

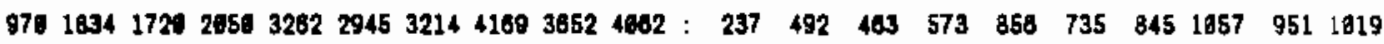


TABLE A.7. Upper Reference Case, Maximum At-Reactor Capacity Plus Transshipment--Projected Annual Storage Requirements

(contd)

N5S日远5

POBL

MIUSTONE 1

PILGRIN 1

CALVET CLF 112

OYSTER CRK 1

PRAIRIE ISL $1 \mathrm{H}$

NORTH ANM 112

SURRY 112

INOIN PT 2

COOPER STN

LIVZICX 1

PEACHEOTTUY 2

PEACHeOTTA 3

LIU GICX 2

PIINT BEACH ILP

BRINSTICK 1

Badisticx 2

ZION its

BVRON 112

BRATDT000 IL

BIO RaCK 1

WAINE YANXEE

FaRT CNHOUN

DAYIS-BESSE 1

LACROSSE

EIRICL FETII-2

SN ONOFRE $1,2,23$

LASNE CTY IL

DRESDEN 2

DRESDEN 3

QUND CITIES 112

HADOAN NECK

ST LUCIE 2

TURKEY PT ILZ

ST LUCIE 1

co0K 112

FITZPATRICK

DUAKE ARNOLD

WISH NUCERR 2

ARK MUCEAR 2

ARK NUCLER 1

PERTY 112

KEAUNEE

\begin{tabular}{|c|c|c|c|c|c|c|c|c|c|c|c|c|c|c|c|c|c|c|c|}
\hline & & & & & & & & & & \\
\hline 140 & 2027 & 2000 & 2609 & 2010 & 2011 & 2012 & 2613 & 2814 & 2915 & $\underline{2040}$ & 2097 & 2608 & 2000 & $\underline{2016}$ & $\underline{2811}$ & 2912 & 2913 & 2014 & 2015 \\
\hline 1 & 175 & 130 & 6 & 178 & 5 & 6 & & . & & & 31 & 24 & a & 31 & 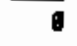 & 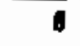 & & & \\
\hline 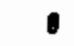 & I & 131 & 1 & 180 & 1 & 168 & 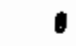 & 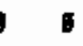 & 6 & & & & 1 & 31 & 1 & 28 & & & \\
\hline 81 & at & 62 & 75 & 81 & 77 & 75 & 78 & 61 & 270 & 30 & 36 & & 28 & 30 & 29 & 28 & 29 & 23 & 195 \\
\hline$\bullet$ & 154 & 117 & 0 & 0 & - & 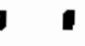 & 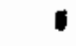 & $=$ & 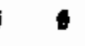 & & 27 & & 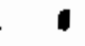 & 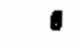 & 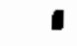 & & & $a$ & \\
\hline 38 & 70 & 54 & 86 & 71 & 60 & 68 & 86 & 140 & 1 & 13 & 25 & & 23 & 25 & 23 & 23 & 23 & 52 & \\
\hline 56 & 56 & 87 & 6 & 113 & 183 & 6 & 109 & 84 & - & 25 & 26 & 40 & - & 52 & 49 & A & 46 & 39 & 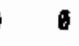 \\
\hline 46 & 46 & 35 & 64 & 45 & 43 & 81 & 156 & & 0 & 21 & 21 & 16 & 39 & 21 & 26 & 37 & 72 & 2 & \\
\hline 5 & $\theta 0$ & 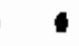 & 55 & $\theta$ & 6 & 54 & b & 45 & 1 & & 27 & & 25 & 27 & 8 & 24 & 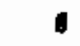 & 29 & \\
\hline 90 & 91 & 73 & 84 & $\infty$ & 84 & 88 & BA & 74 & 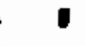 & 10 & 17 & 13 & 15 & 18 & 15 & 10 & 15 & 13 & \\
\hline 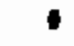 & 187 & 140 & 5 & 188 & 179 & 6 & 177 & 147 & 1 & 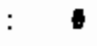 & 33 & 26 & 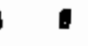 & 33 & 32 & 0 & 31 & 20 & \\
\hline 281 & 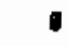 & 150 & 191 & 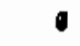 & 169 & 187 & 0 & 0 & 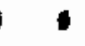 & 35 & & 20 & 34 & 1 & 34 & 33 & 0 & 6 & 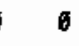 \\
\hline 189 & 286 & 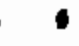 & 181 & 189 & 6 & 177 & 177 & 156 & 1 & 33 & 33 & & 32 & 34 & 1 & 32 & 31 & 28 & 8 \\
\hline 1) & 255 & 1 & 227 & 168 & a & 216 & 0 & 183 & 204 & : & 45 & 1 & 40 & 20 & 1 & 38 & 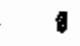 & 32 & 36 \\
\hline 57 & 55 & 44 & 56 & 57 & 148 & 25 & 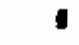 & 1 & $\theta$ & 20 & 20 & 16 & 18 & 20 & 52 & - & 6 & 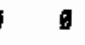 & $\theta$ \\
\hline 161 & 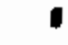 & 120 & $\bullet$ & 0 & 0 & $\Delta$ & 0 & 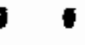 & $t$ & 30 & 0 & 24 & 1 & 0 & 0 & - & 6 & 8 & 8 \\
\hline - & 102 & 120 & 1 & 153 & 153 & 1 & 152 & 126 & 1 & 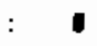 & 31 & 24 & 0 & 20 & 29 & 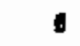 & 28 & 24 & \\
\hline 84 & 83 & 88 & 61 & 83 & 120 & 59 & 68 & 241 & e & 29 & 29 & 5 & 28 & 29 & 55 & 27 & 27 & 110 & \\
\hline 73 & 63 & 99 & 76 & 58 & 131 & 89 & 56 & 100 & 58 & 31 & 27 & 42 & 39 & 25 & 55 & 29 & 24 & 45 & 25 \\
\hline 131 & 128 & 0 & 127 & 118 & - & 128 & 112 & 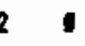 & 103 & 56 & 54 & 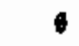 & 54 & 49 & 6 & 53 & 47 & 0 & 44 \\
\hline - & & & 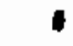 & & ฮ & 1 & & 1 & & & & 0 & 1 & & 0 & 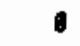 & c & 8 & 6 \\
\hline 63 & 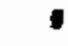 & 0 & 50 & b & 80 & 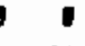 & 6 & $\bullet$ & - & 24 & 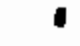 & 19 & 22 & \rfloor & 23 & 1 & 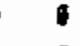 & 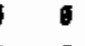 & 6 \\
\hline 39 & 1 & 24 & 36 & 1 & 36 & 34 & 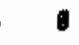 & 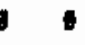 & 1 & 14 & 1 & 10 & 13 & 0 & 13 & 12 & $\theta$ & - & $\theta$ \\
\hline 53 & 与 & 41 & 50 & $\bullet$ & 54 & 50 & 1 & 41 & 42 & & & & 3 & & 23 & 23 & 1 & 19 & 19 \\
\hline 21 & 5 & 16 & 6 & 6 & 0 & 0 & 6 & 1 & 0 & 3 & 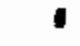 & 3 & 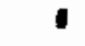 & 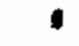 & - & 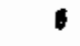 & $\theta$ & I & 8 \\
\hline 0 & 262 & 0 & 247 & 252 & a & 238 & $\sigma$ & 197 & $\boldsymbol{1}$ & 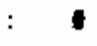 & 46 & 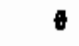 & 45 & 48 & 6 & 43 & 0 & 38 & 6 \\
\hline 281 & 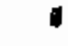 & 157 & 231 & 78 & 75 & 73 & 74 & 82 & 69 & 83 & 6 & 85 & $n$ & 39 & 32 & 31 & 32 & 26 & 27 \\
\hline 194 & 392 & 147 & 185 & 382 & 182 & 178 & 384 & 149 & 150 & 35 & 70 & 27 & 34 & 70 & 33 & 32 & 88 & 27 & 28 \\
\hline 137 & 137 & & 135 & 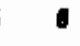 & - & 0 & 0 & ( & 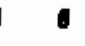 & 20 & 23 & & 23 & 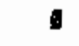 & 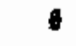 & 1 & 6 & 6 & 8 \\
\hline 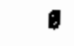 & 137 & 107 & 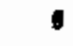 & 137 & 131 & 0 & $\theta$ & 6 & 1 & & 23 & 18 & $\theta$ & 23 & 22 & & 0 & 0 & $\theta$ \\
\hline 140 & 278 & 168 & 137 & 278 & 134 & 133 & 285 & 712 & 5 & 25 & 49 & 19 & 24 & 49 & 24 & 23 & 47 & 125 & 0 \\
\hline 48 & 40 & ( & 0 & 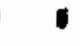 & 5 & 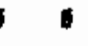 & 6 & 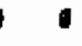 & 6 & 19 & 10 & & 1 & - & 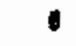 & 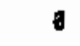 & b & 0 & 6 \\
\hline$\theta$ & 43 & 46 & 0 & 63 & 80 & $\theta$ & 50 & 49 & 0 & & 25 & 19 & 1 & 25 & 23 & I & 23 & 19 & 6 \\
\hline 62 & 42 & 33 & 71 & 41 & 30 & 70 & 185 & 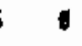 & 1 & 38 & 10 & 15 & 32 & 19 & 17 & 32 & 96 & 6 & 6 \\
\hline 68 & 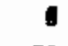 & 51 & 61 & & 82 & 61 & 0 & 49 & 53 & & 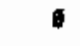 & 1 & 23 & 6 & 24 & 23 & 6 & 19 & 26 \\
\hline 69 & 70 & 115 & 67 & 77 & 69 & 72 & 139 & 54 & 253 & 32 & 31 & 59 & 31 & 31 & 30 & 29 & 59 & 25 & 113 \\
\hline 153 & 8 & 122 & 146 & 6 & 140 & 142 & $\theta$ & 120 & 122 & 27 & 0 & & 28 & 9 & 28 & 25 & 8 & 21 & 22 \\
\hline 180 & 0 & 82 & $\infty$ & 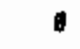 & 100 & 99 & 8 & 83 & 86 & 1 & . & & 18 & $\emptyset$ & 18 & 18 & 0 & 15 & is \\
\hline 139 & 139 & 100 & 135 & 141 & 133 & 131 & 131 & 199 & 113 & 24 & 24 & & 24 & 25 & 23 & 23 & 23 & 19 & 20 \\
\hline 1 & 59 & 47 & 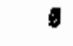 & 59 & $\theta$ & 55 & 57 & 6 & 45 & • & 24 & 20 & 0 & 24 & g & 23 & 24 & 0 & 19 \\
\hline 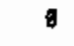 & 56 & - & 47 & 1 & 47 & 47 & 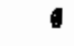 & 39 & 0 & & & & 22 & 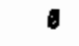 & 22 & 22 & 0 & 18 & $\theta$ \\
\hline 490 & 140 & 185 & 459 & 181 & 232 & 436 & 137 & 211 & 150 & . & 27 & 0 & 93 & 29 & 42 & 78 & 25 & 38 & 27 \\
\hline 33 & 33 & 25 & 30 & 32 & 31 & 31 & 29 & 25 & 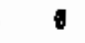 & 13 & 10 & & 11 & 12 & 12 & 12 & 11 & 9 & \\
\hline
\end{tabular}


TABLE A.7. Upper Reference Case, Maximum At-Reactor Capacity Plus Transshipment--Projected Annual Storage Requirements (contd)

POOL

SNEI 1

SHEY UAIT 2

CLINTON 1

aimu

RAKHO SECO- 1

VT YAMEE 1

HATCH 1 L2

SUSRUgaNM 112

B VAUEY 2

B YNLEY 1

BODTIS FERT1L2

BRTIS FERRY3

PNO VERE 2

PMLO VERDE 1

PALD VGOE 3

CiTSTH RMR 3

VOOTE $1 \mathrm{~L}$

TROJAN

INDIAN PT 3

SERUOYAH 12

TATTS EAR IL

Ba FFONTE 1

B日FONTE 2

CNUATAY 1

HAPRIS 1

RDEINSON 2

RVA BEN 1

TATERORO 3

MNTICE

FNPLEY 2

FAPLEY 1

MISTONE 2

MILSTONE 3

DIABD CAYYON-1

OIARO CANYON-2

NINE UILE PT2

ICCUIRE 1

ACONEE IL

OCANEE 3

ICOUIRE 2

CATATER !

CATANGA 2
NSSEALIES

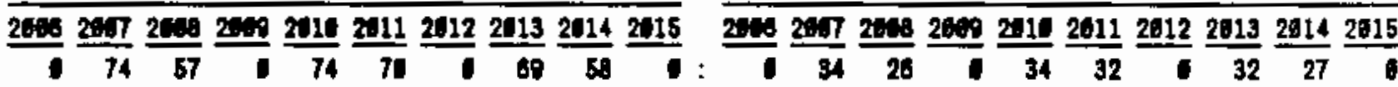

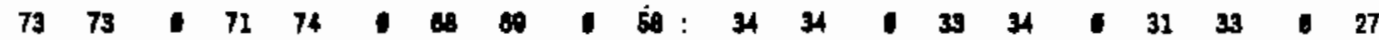

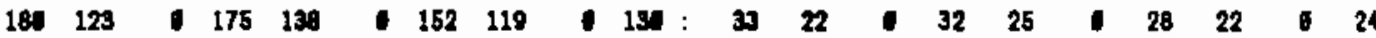

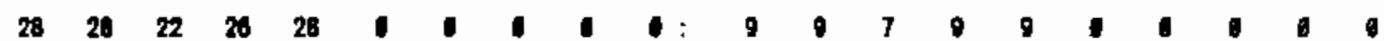

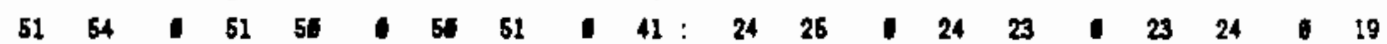

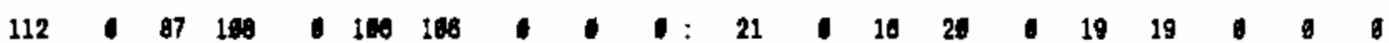

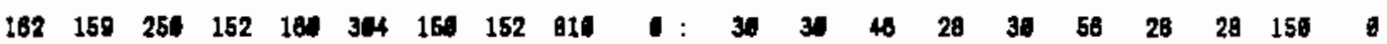

$\begin{array}{lllllllllllllllllllll}197 & 195 & 369 & 191 & 195 & 372 & 180 & 186 & 396 & 159 & : & 34 & 34 & 53 & 33 & 34 & 84 & 32 & 32 & 53 & 27\end{array}$

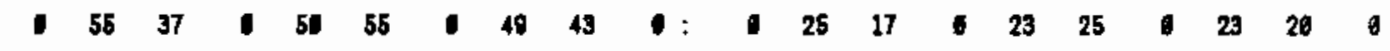

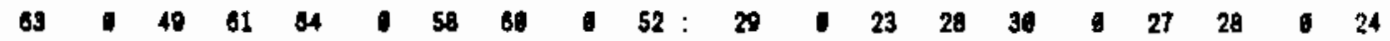

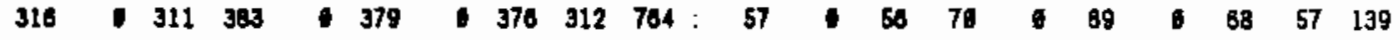

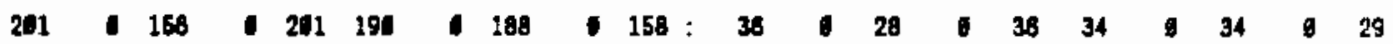

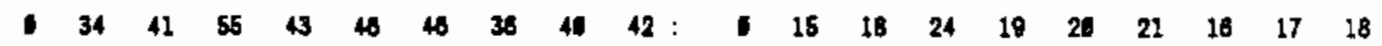

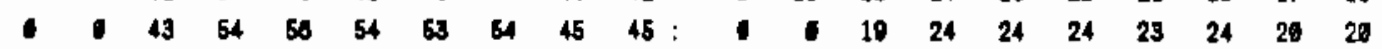

$\begin{array}{llllllllllllllllllll} & 48 & 32 & 59 & 45 & 49 & 48 & 45 & 39 & 41: & 1 & 21 & 14 & 23 & 29 & 21 & 21 & 29 & 17 & 18\end{array}$

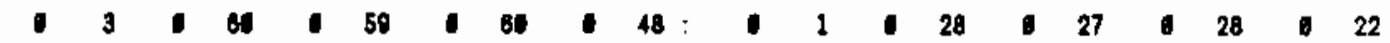

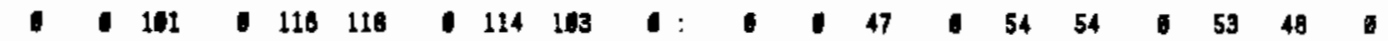

$\begin{array}{llllllllllllllllllll}-31 & 40 & 42 & 40 & 40 & 40 & 33 & 34 & 10 & 18 & 18 & 18 & 18 & 18 & 18 & 18 & 15 & 18\end{array}$

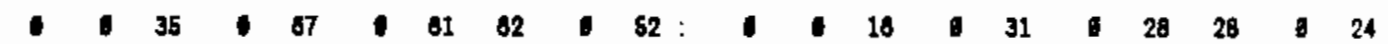

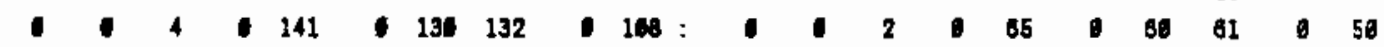

\begin{tabular}{llllllllllllllllllll}
\hline & 49 & 139 & 53 & 58 & 59 & 45 & 192 & 49 & 19 & 13 & 04 & 24 & 27 & 27 & 21 & 47 & 23
\end{tabular}

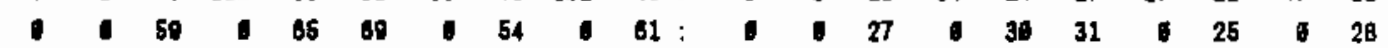

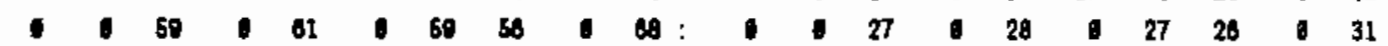

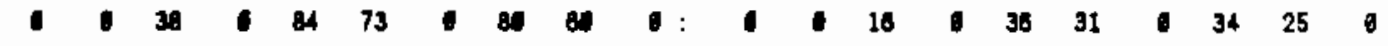

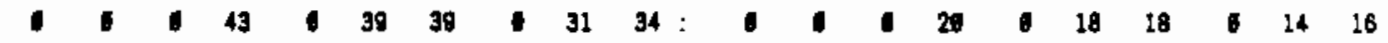

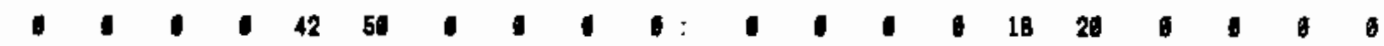

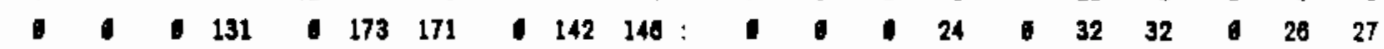

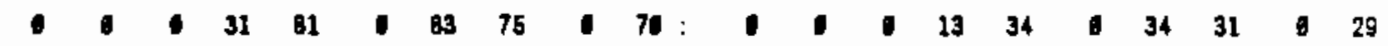

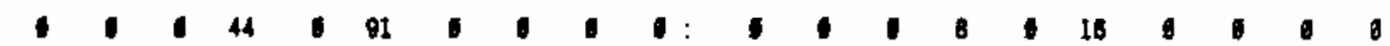

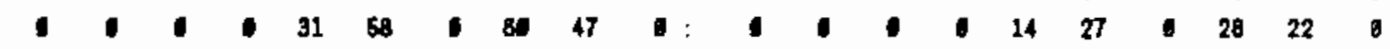

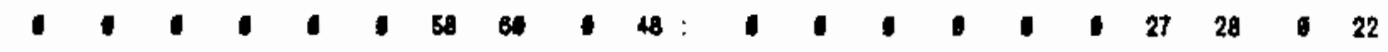

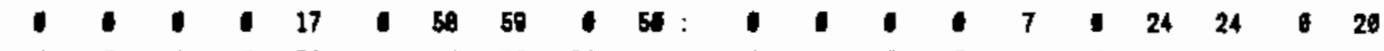

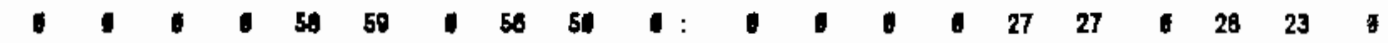

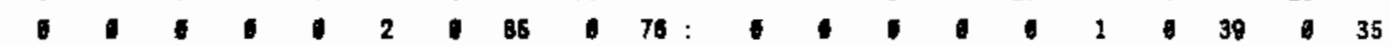

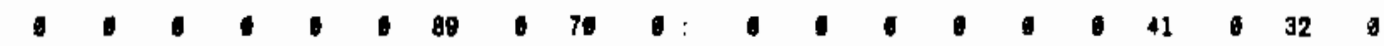

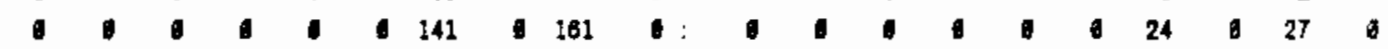

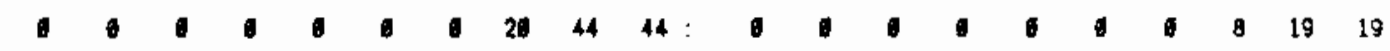

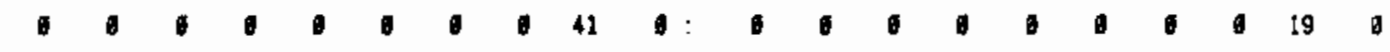

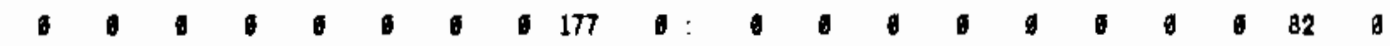

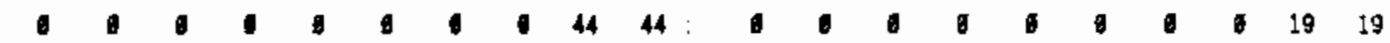

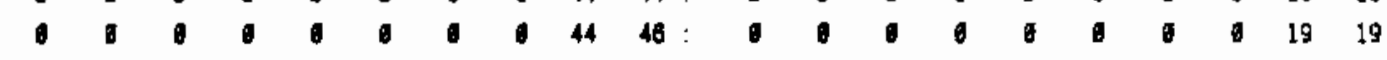


TABLE A.7. Upper Reference Case, Maximum At-Reactor Capacity Plus Transshipment--Projected. Annual Storage Requirements (contd)

NSSERTES

PDOL

HOPE CREXX

TUF CREEX 1

SUNGR 1

COUNCHE PK 142

SHQREHA

PTR TOTAL

GUR TOTAL

TOTAL

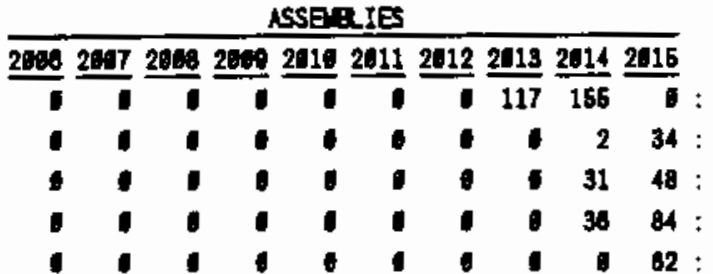

\begin{tabular}{|c|c|c|c|c|c|c|c|c|c|}
\hline \multicolumn{10}{|c|}{ पTIHU } \\
\hline 20as & $\underline{20,7}$ & 2000 & 2000 & 2010 & 2011 & 2012 & 2013 & 2914 & 2815 \\
\hline 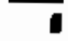 & & 1 & 1 & 5 & 6 & 7 & 21 & 28 & 8 \\
\hline 6 & ॥ & 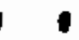 & ઇ & 0 & 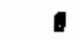 & 0 & - & 1 & 18 \\
\hline D & 1 & 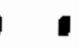 & 0 & 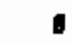 & 0 & 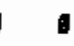 & 6 & 14 & 22 \\
\hline 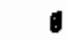 & 1 & 0 & 1 & $\bullet$ & 1 & 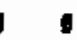 & 9 & 15 & 34 \\
\hline ฮ & ง & $B$ & $\exists$ & $\theta$ & 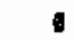 & 9 & 0 & 0 & 11 \\
\hline
\end{tabular}

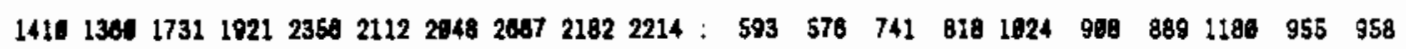

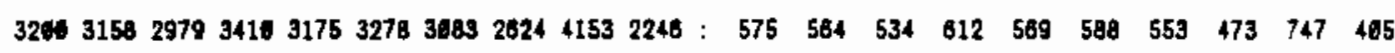
$4010451947105331553153945131529163954461: 1168 \quad 1140127514391593149614421653 \quad 1792 \quad 1363$ 
TABLE A.7. Upper Reference Case, Maximum At-Reactor Capacity Plus Transshipment--Projected Annual Storage Requirements (contd)

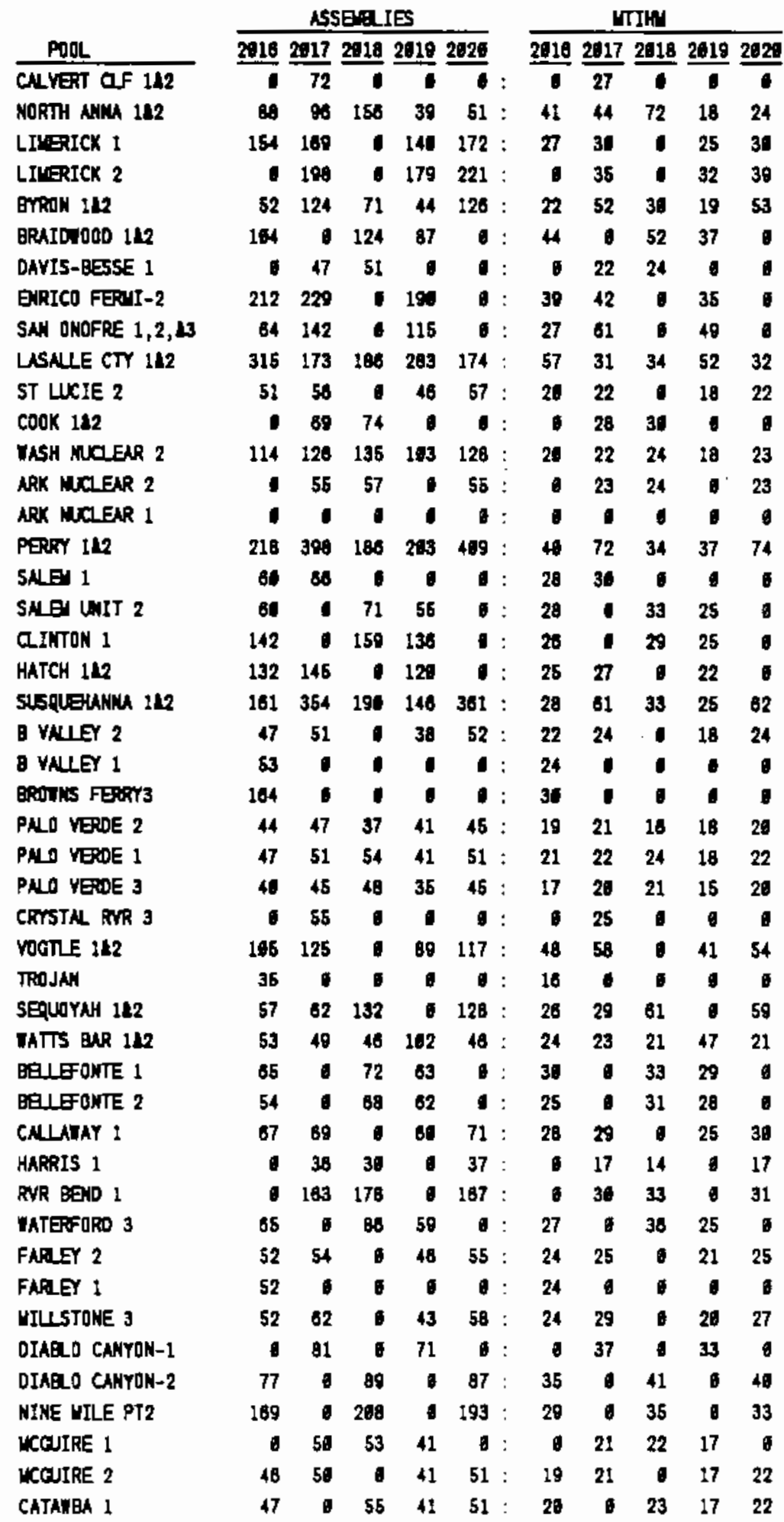


TABLE A.7. Upper Reference Case, Maximum At-Reactor Capacity Plus Transshipment--Projected Annual Storage Requirements (contd)

\begin{tabular}{|c|c|c|c|c|c|c|c|c|c|c|}
\hline \multirow[b]{2}{*}{ Pou } & \multicolumn{5}{|c|}{ ASSEQIES } & \multicolumn{5}{|c|}{ WTIH } \\
\hline & $2016 ?$ & 2017 & 2018 & 2019 & 2020 & 2110 & 2117 & 218 & 2619 & 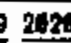 \\
\hline CTTANEA 2 & 40 & 48 & $\because$ & 34 & 45 & 17 & 2 & $\pi$ & 14 & 18 \\
\hline HOPE CREX & 164 & 183 & - & 199 & 187 & 39 & 39 & - & 25 & 34 \\
\hline DOUF CREAR 1 & 34 & 30 & 41 & 31 & s3 & 16 & 18 & 19 & 14 & 18 \\
\hline sung 1 & - & 69 & 56 & 1 & 54 & - & 25 & 26 & - & 25 \\
\hline COWNCHE PK 182 & 83 & 95 & 89 & 76 & 93 & 33 & 39 & 36 & 31 & 37 \\
\hline SHOREGW & - & 16 & 134 & 0 & 132 & ! & 10 & 24 & $\theta$ & 24 \\
\hline$M M P-1$ & 78 & $\bullet$ & 96 & 81 & $\theta$ & 36 & 0 & 39 & 36 & - \\
\hline NWP-3 & 59 & $\omega$ & 58 & G & 80 & 28 & 28 & 26 & 24 & 34 \\
\hline SEABROOK 2 & a & 0 & a & 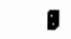 & 17 & . & 6 & - & 6 & B \\
\hline PAR TOTN & 1831 & 1999 & $17: 2$ & 1534 & 1490 & 811 & 836 & 751 & 674 & 881 \\
\hline BiR TOTAL & 1945 & 2242 & 1978 & 1638 & 2144 & 369 & 403 & 248 & 295 & 392 \\
\hline TOTN & 3778 & 4150 & 3.78 & 3173 & 3643 & 1161 & 1230 & 998 & 970 & 1044 \\
\hline
\end{tabular}


TABLE A.8. Upper Reference Case, Maximum At-Reactor Capacity Plus Transshipment--Projected Cumulative Storage Requirements

NSSBDIES

MTIN

POOL

PALISADES

UIUSTONE 1

PILORIU 1

CALERT QF 110

OYSTER CPK 1

PQAIRIE ISL 112

NORTH NNA IL2

SURRT 112

INDIAN PT 2

COQPER STM

LIVERTCX I

LINGRICX 2

\begin{tabular}{|c|c|c|c|c|c|c|c|c|c|}
\hline 1986 & 1097 & 1988 & 1989 & 1990 & 1991 & 1992 & 1903 & 1994 & 1995 \\
\hline 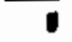 & 4 & 4 & 82 & 129 & 120 & 191 & 101 & 238 & 238 \\
\hline . & B8 & 86 & 266 & 250 & 448 & 448 & 815 & 782 & 782 \\
\hline 6 & . & 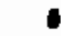 & 0 & 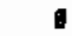 & 0 & 20 & 68 & 233 & 233 \\
\hline t & 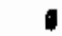 & 1 & . & 0 & 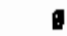 & 0 & 20 & 97 & 178 \\
\hline 0 & 0 & 6 & 9 & 6 & 0 & - & 9 & 30 & 38 \\
\hline 1 & 1 & 0 & - & 1 & 6 & 6 & D & 6 & 76 \\
\hline 0 & 6 & 0 & 0 & 5 & 1 & 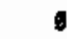 & 6 & 51 & 188 \\
\hline 1 & 1 & 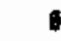 & 0 & 1 & 1 & 0 & 9 & G & 94 \\
\hline 6 & 0 & 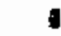 & 9 & 8 & 1 & $g$ & 1 & 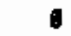 & 15 \\
\hline 1 & 0 & 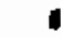 & $\bullet$ & 0 & 5 & 8 & 0 & 0 & 10 \\
\hline 6 & 0 & 0 & 6 & 8 & : & 0 & 0 & 0 & 44 \\
\hline 6 & 0 & 0 & 1 & 9 & 1 & 1 & 0 & 6 & 217 \\
\hline
\end{tabular}

$\frac{1988}{1997} \frac{1988}{2} \frac{1989}{24} \frac{1994}{47} \frac{1991}{47} \frac{1992}{71} \frac{1993}{71} \frac{1994}{93} \frac{1995}{93}$

$\begin{array}{llllllllll}6 & 15 & 15 & 45 & 45 & 80 & 86 & 169 & 139 & 139\end{array}$

60

10

6

1. 0.0 .027

- . . . .0 .2450

1. .0 .90 .93

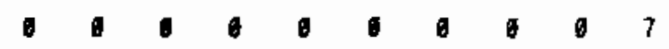

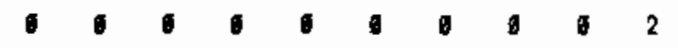

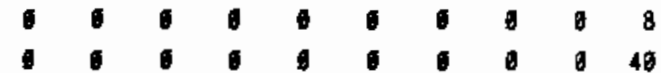

PTR TOTN

$\begin{array}{llllllllllllllllllll}4 & 4 & 4 & 82 & 12 & 120 & 181 & 201 & 392 & 709: & 0 & 2 & 2 & 24 & 47 & 47 & 71 & 78 & 155 & 286\end{array}$

GIR TOTNL

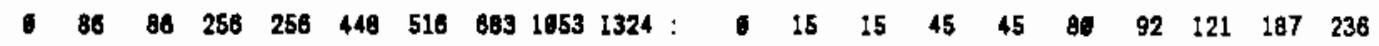

TOTM

$\begin{array}{llllllllll}\text { - } & 90 & 98 & 318 & 376 & 588 & 697 & 884 & 1445 & 2933\end{array}$

$\begin{array}{llllllllll}9 & 17 & 17 & 70 & 92 & 128 & 162 & 199 & 342 & 523\end{array}$ 
TABLE A.8. Upper Reference Case, Maximum At-Reactor Capacity PTus Transshipment--Projected Cumulative Storage Requirements (contd)

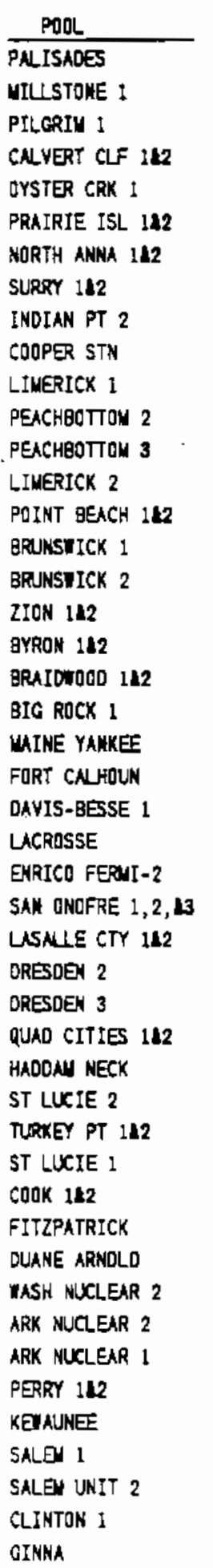

\begin{tabular}{|c|c|c|c|c|c|c|c|c|c|c|c|c|c|c|c|c|c|c|c|}
\hline 1996 & 1997 & 1998 & 1999 & 2609 & 2001 & 2012 & 2043 & 204 & 2005 & 1900 & 1997 & 1998 & 190 & 2009 & 2001 & 2012 & 2893 & 2904 & 2065 \\
\hline 238 & $\overrightarrow{238}$ & 238 & $\overline{230}$ & 238 & $\overline{238}$ & 238 & 239 & 239 & 238 & 93 & 83 & 93 & 93 & 93 & 93 & 93 & 93 & 93 & 93 \\
\hline 931 & 981 & 1004 & 1084 & 1265 & 1425 & 1425 & 1895 & 1895 & 1746 & 186 & 180 & 195 & 195 & 225 & 263 & 253 & 285 & 285 & 310 \\
\hline 233 & 397 & 387 & 590 & 530 & 895 & 695 & 868 & 868 & 1843 & 41 & 68 & 88 & 95 & 95 & 123 & 123 & 153 & 153 & 177 \\
\hline 248 & 319 & 393 & 485 & 543 & 615 & 680 & 74 & 40 & 894 & 93 & 120 & 148 & 175 & 204 & 231 & 258 & 287 & 316 & 340 \\
\hline 168 & 288 & 288 & 418 & 416 & 555 & 876 & 878 & 823 & 942 & $30^{\circ}$ & 51 & 51 & 73 & 73 & 98 & 119 & 119 & 145 & 166 \\
\hline 105 & 169 & 233 & 290 & 304 & 398 & 452 & 525 & 591 & 47 & 37 & 61 & 93 & 105 & 129 & 148 & 169 & 188 & 299 & 229 \\
\hline 295 & 257 & 309 & 409 & 464 & 515 & 686 & 623 & 710 & Bes & 95 & 118 & 143 & 180 & 214 & 238 & 280 & 386 & 331 & 372 \\
\hline 133 & 176 & 282 & $3: 3$ & 347 & 430 & 487 & 615 & 812 & 638 & 81 & B1 & 129 & 139 & 159 & 197 & 214 & 236 & 278 & 293 \\
\hline 15 & 69 & 69 & 123 & 181 & 181 & 229 & 229 & 280 & 334 & 7 & 31 & 31 & 68 & A2 & 62 & 103 & 193 & 129 & 151 \\
\hline 96 & 186 & 28 & 367 & 463 & 551 & 636 & 731 & 823 & 890 & 17 & 34 & 51 & 87 & 84 & 180 & 115 & 133 & 156 & 164 \\
\hline 205 & 295 & 380 & 559 & 550 & 721 & 974 & 874 & 1664 & 1284 & 36 & 38 & B7 & 89 & 98 & 128 & 155 & 155 & 187 & 213 \\
\hline 100 & 352 & 352 & 531 & 728 & 720 & 887 & 1002 & $1 \operatorname{mon}^{2}$ & 1250 & 30 & 82 & 62 & 94 & 129 & 128 & 157 & 194 & 194 & 222 \\
\hline G & 172 & 349 & 349 & S31 & 703 & $7: 3$ & 097 & 1070 & 1076 & ต & 34 & 62 & 62 & 94 & 125 & 125 & 159 & 191 & 191 \\
\hline 217 & 501 & 581 & 875 & 980 & 988 & 1689 & 1080 & 1325 & 1435 & 40 & 92 & 92 & 123 & 104 & 184 & 198 & 198 & 238 & 257 \\
\hline 42 & 93 & 144 & 194 & 249 & 290 & 344 & 401 & 454 & 490 & 15 & 33 & 5i & 89 & 88 & 106 & 122 & 142 & 161 & 177 \\
\hline B & 139 & 287 & 287 & 445 & 445 & 576 & 743 & 743 & 872 & 5 & 20 & 54 & 54 & 83 & 83 & 168 & 139 & 139 & 163 \\
\hline 0 & & 150 & 295 & 295 & 442 & 573 & 573 & 727 & 850 & 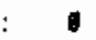 & 1 & 28 & 56 & 55 & 93 & 167 & 107 & 136 & 160 \\
\hline 0 & 28 & 80 & 199 & 291 & 317 & 419 & 495 & 546 & 840 & 6 & 13 & 39 & 91 & 119 & 146 & 191 & 222 & 249 & 295 \\
\hline 5 & 67 & 135 & 252 & 324 & 398 & 545 & 581 & 948 & 763 & 1 & 28 & 57 & 107 & 137 & 168 & 214 & 246 & 274 & 319 \\
\hline 6 & 171 & 386 & 308 & 457 & 612 & 692 & 724 & 958 & 860 & 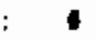 & 72 & 179 & 129 & 193 & 255 & 255 & 396 & 363 & 363 \\
\hline b & 2 & IB & 39 & 51 & 67 & 67 & 85 & 101 & $\$ 15$ & 6 & 6 & 2 & 4 & 7 & 9 & 9 & 11 & 13 & 15 \\
\hline 0 & 8 & 8 & 65 & 128 & 128 & IBA & 247 & 247 & 290 & 0 & 3 & 3 & 25 & 49 & 49 & 68 & 94 & 94 & 113 \\
\hline a & 34 & 34 & 67 & 165 & 105 & 138 & 17 & 17 & 27 & 0 & 12 & 12 & 24 & 38 & 36 & 49 & 84 & $B 4$ & 75 \\
\hline 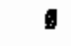 & 47 & 47 & 94 & 148 & 146 & 289 & 243 & 243 & 2855 & 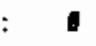 & 22 & 22 & 44 & 88 & 88 & 88 & 114 & 114 & 133 \\
\hline 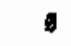 & 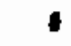 & 12 & 12 & 33 & 33 & 48 & 40 & $\theta 0$ & 89 & 1 & ฮ & 2 & 2 & 5 & 5 & $g$ & $B$ & 11 & $\$ 1$ \\
\hline 0 & 6 & 130 & 139 & 377 & 377 & 583 & 842 & 42 & 1048 & 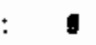 & 1 & 24 & 24 & 89 & 89 & 106 & 153 & 153 & 190 \\
\hline 6 & 5 & 65 & 128 & 249 & 324 & 426 & 51 & 619 & 682 & 1 & 1 & 28 & 54 & 102 & 133 & 173 & 268 & 258 & 283 \\
\hline 6 & 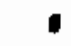 & 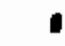 & 160 & 294 & 644 & 800 & 90 & 1306 & 1518 & 1 & 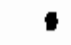 & 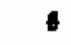 & 19 & 54 & 117 & 148 & 182 & 248 & 276 \\
\hline$\theta$ & 0 & 1 & ฮ & 135 & 200 & 290 & $4 \mathbf{2}$ & 534 & 634 & 6 & 5 & 4 & 6 & 23 & 44 & 44 & 87 & 89 & 89 \\
\hline 9 & 0 & 0 & 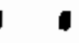 & 5 & 125 & 237 & 237 & 368 & $4 \pi$ & 5 & 6 & 0 & $\bullet$ & 6 & 21 & 40 & 40 & 62 & 80 \\
\hline 1 & 1 & ॥ & 1 & 136 & 391 & s4 & 849 & 018 & 1020 & $\bullet$ & 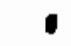 & I & 6 & 24 & $\infty 0$ & 89 & 114 & 161 & 181 \\
\hline 1 & 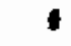 & 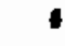 & 21. & 29 & 62 & 160 & 148 & 148 & 185 & 0 & 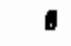 & 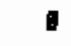 & 8 & 8 & 26 & 41 & 61 & 81 & 76 \\
\hline 1 & 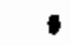 & 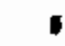 & $n$ & 27 & 85 & 130 & 130 & 190 & 240 & 0 & 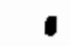 & - & 11 & 11 & 33 & 53 & $\mathbf{5 3}$ & 76 & 96 \\
\hline 5 & 5 & $\bullet$ & $\bullet$ & 41 & 79 & 113 & 201 & 241 & 274 & 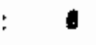 & 1 & 1 & 1 & 19 & 36 & 52 & 92 & 111 & 126 \\
\hline 6 & 0 & - & 1 & $\infty$ & 68 & 120 & 189 & 189 & 242 & $\theta$ & 1 & 0 & 1 & 25 & 25 & 46 & 72 & 72 & 92 \\
\hline g & 0 & - & 4 & 147 & 211 & 273 & 346 & 419 & 537 & $B$ & 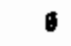 & 8 & 2 & 83 & 93 & 118 & 151 & 181 & 232 \\
\hline 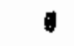 & 1 & 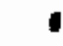 & 32 & 182 & 182 & 380 & 485 & 485 & 588 & 6 & ๑ & 6 & 6 & 32 & 32 & 54 & 83 & 83 & 104 \\
\hline a & 8 & 1 & 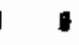 & 34 & 34 & 120 & 228 & 228 & 317 & 6 & g & 0 & 0 & 6 & 6 & 21 & 41 & 41 & 56 \\
\hline 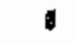 & 8 & 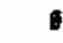 & 0 & 133 & 261 & 374 & 519 & 653 & 704 & a & 9 & 9 & - & 23 & 40 & 66 & 91 & 115 & 135 \\
\hline 5 & 6 & 0 & g & 1 & 18 & 18 & 89 & 60 & 127 & I & 1 & 6 & - & 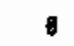 & 7 & 7 & 33 & 33 & 53 \\
\hline 0 & 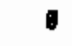 & - & 1 & 1 & 0 & 30 & 30 & 85 & $\mathbf{t 2 5}$ & 6 & 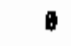 & 1 & 1 & $\theta$ & 0 & 18 & 18 & 39 & 58 \\
\hline 0 & 0 & I & - & 1 & 42 & 242 & $\pi \theta$ & 941 & 1068 & 1 & a & 0 & a & 1 & 8 & 44 & 142 & 171 & 194 \\
\hline 1 & -1 & - & $\theta$ & 1 & 16 & 44 & 78 & 109 & 135 & 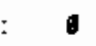 & 1 & 0 & 0 & a & 7 & 17 & 30 & 41 & 51 \\
\hline H & $\theta$ & 0 & 0 & 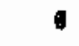 & 1 & 58 & 58 & 120 & 179 & a & d & 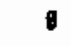 & • & 0 & 0 & 23 & 23 & 55 & 82 \\
\hline 6 & 0 & 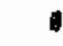 & 0 & $\theta$ & 5 & 6 & $\pi$ & 147 & 147 & 8 & 6 & 6 & 0 & 0 & 1 & 1 & 35 & 67 & 67 \\
\hline 9 & 1 & ง & 8 & 5 & 0 & . & 58 & 199 & 198 & 6 & 5 & 6 & 1 & 0 & 9 & g & 11 & 36 & 36 \\
\hline 1 & t & . & 1 & 0 & 6 & 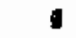 & 26 & 53 & 76 & 0 & 9 & 6 & t & $\theta$ & 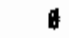 & G & 9 & 17 & 25 \\
\hline
\end{tabular}


TABLE A.8. Upper Reference Case, Maximum At-Reactor Capacity Plus Transshipment--Projected Cumulative Storage Requirements (contd)

POOL RUCHO SECO-1 VT YANKEE 1 HATCH 112 SISQUEEANIT 112 G VNAY 2

NSSEIRIES

\begin{tabular}{|c|c|c|c|c|c|c|c|c|c|c|c|c|c|c|c|c|c|c|c|}
\hline$\infty 8$ & 1807 & 1900 & 1900 & $2 \mathrm{ara}$ & 2011 & 2012 & 2643 & 2044 & 2045 & 1000 & 1997 & 1998 & 1999 & 2ang & 2901 & 2692 & 2863 & 2964 & 2605 \\
\hline 0 & 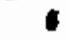 & & 0 & $\theta$ & 0 & 1 & 1 & 51 & 51 & & 0 & $\boldsymbol{1}$ & 1 & 0 & 1 & 6 & 1 & 24 & 24 \\
\hline 1 & 1 & $\bullet$ & 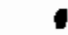 & 1 & 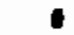 & $\boldsymbol{0}$ & 74 & 74 & 162 & (1) & t & 1 & ๘ & $\theta$ & • & 0 & 14 & 14 & 30 \\
\hline 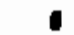 & 1 & 0 & 」 & t & 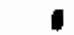 & 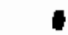 & 0 & 26 & 283 & 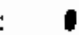 & ॥ & 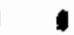 & 0 & 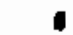 & 6 & 6 & 1 & 5 & 53 \\
\hline 1 & 1 & 1 & 0 & d & 1 & 0 & 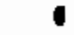 & 30 & 342 & 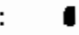 & 1 & 9 & 1 & $\theta$ & . & a & 6 & 5 & 59 \\
\hline 6 & 1 & 1 & - & 0 & - & 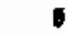 & 0 & 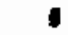 & 1 & ๑ & ต & 0 & 1 & ๑ & 6 & v & 0 & $\theta$ & 6 \\
\hline
\end{tabular}

PIR TOTN

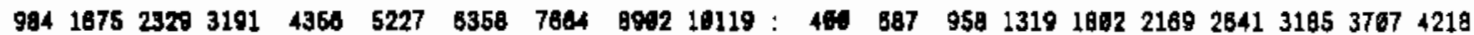

BIR TOTN $2919316242285416 \quad 7513 \quad 9587 \quad 1167914533 \quad 16947 \quad 19792: 386 \quad 568 \quad 758 \quad 9701343 \quad 1711 \quad 2684 \quad 2597 \quad 3826 \quad 3534$ TOTN

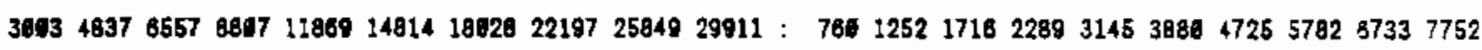


TABLE A.8. Upper Reference Case, Maximum At-Reactor Capacity Plus

Transshipment--Projected Cumalative Storage Requirements

(contd)

NSSERIES

UTIMI

\begin{tabular}{|c|c|c|c|c|c|c|c|c|c|c|c|c|c|c|c|c|c|c|c|c|}
\hline & & & & & 10 & 11 & 12 & 13 & 114 & 116 & 2008 & 97 & 2698 & 2909 & 2010 & 2011 & 2112 & 2013 & 2914 & 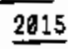 \\
\hline LISADES & 38 & 238 & 238 & 238 & 238 & 238 & 238 & 238 & 238 & 238 & 93 & 93 & 93 & 93 & 93 & 93 & 93 & 93 & 93 & 93 \\
\hline LSTONE 1 & 1745 & 1924 & 2658 & 2060 & 2232 & 2232 & 2232 & 2232 & 2232 & 2232 & 316 & 341 & 368 & 368 & 307 & 397 & 397 & 397 & 397 & 397 \\
\hline LERIN 1 & 603 & 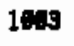 & 134 & 134 & 345 & 303 & 481 & 1481 & 401 & 1461 & 17 & 17 & 260 & 294 & 230 & 230 & 258 & 258 & 258 & 258 \\
\hline LYERT QF 112 & 985 & 1085 & 127 & 1292 & 1289 & 386 & 435 & 1511 & 572 & 1851 & 371 & 401 & 424 & 452 & 483 & 512 & 540 & & 91 & 697 \\
\hline STER CRK 1 & 942 & 1006 & 213 & 213 & 213 & 13 & 213 & 1213 & 213 & 1213 & 108 & 194 & 214 & 214 & 214 & 214 & 214 & 214 & 14 & 214 \\
\hline AIRIE ISL 1 & OB3 & 753 & 97 & 972 & 943 & $10 \%$ & 1075 & 1141 & 289 & 1289 & 242 & 267 & 286 & 309 & 334 & 358 & 381 & 484 & 157 & 457 \\
\hline RTH NWA & 881 & 917 & 1604 & 1004 & $\$ 117$ & 1223 & 1223 & 1323 & 407 & $14 \pi$ & 398 & 424 & 464 & 464 & 516 & $\mathbf{5 8 5}$ & 565 & & 50 & 850 \\
\hline PRY 112 & 684 & 731 & 765 & 840 & 394 & 937 & 1817 & 1173 & 1173 & 1173 & 314 & 335 & 351 & 391 & 410 & 430 & 487 & 38 & 38 & 538 \\
\hline IDIAN PT 2 & 334 & 394 & 394 & 449 & $\operatorname{sen}$ & 56 & 563 & 563 & 948 & 848 & 151 & 178 & 178 & 293 & 230 & 230 & 254 & 54 & 74 & 274 \\
\hline IDPER S & 997 & 1088 & 1161 & 1245 & 1344 & 1428 & 1518 & 1600 & 1874 & 1974 & 182 & 198 & 212 & 227 & 245 & 289 & 276 & 292 & 85 & 325 \\
\hline WERICK 1 & 1294 & 1391 & $\$ 537$ & 1537 & 1723 & 1922 & 1942 & 2079 & 2226 & 2226 & 213 & 247 & 272 & 272 & 305 & 137 & 337 & 369 & 95 & 395 \\
\hline JRCHBOTTOH 2 & 1450 & 1450 & 184s & 1797 & 1797 & 1986 & 2173 & 2173 & 2173 & 2173 & 257 & 257 & 285 & 319 & 319 & 352 & 385 & & 85 & 385 \\
\hline HCHBOTTOY 3 & 1264 & $146:$ & 1451 & 1631 & 1820 & 1920 & 197 & 2174 & 2334 & 2330 & 224 & 257 & 257 & 298 & 323 & 23 & 354 & 35 & 13 & 413 \\
\hline INERICK 2 & 1435 & 1894 & 1680 & 1817 & 2975 & 2975 & 2281 & 2291 & 2474 & 2678 & 257 & 393 & 393 & 343 & 371 & 371 & 409 & 469 & 42 & 478 \\
\hline JINT BEACH & 550 & 611 & 655 & 705 & 782 & 948 & 293 & 293 & 933 & 293 & 197 & 217 & 233 & 251 & 271 & 322 & 331 & & 81 & 331 \\
\hline unsticx 1 & 1933 & 1853 & 1159 & 1160 & 1150 & 1158 & 1259 & 1159 & 1159 & 1159 & 193 & 193 & 217 & 217 & 217 & 217 & 217 & 17 & 17 & 217 \\
\hline DNSTICX 2 & 856 & 1018 & 1144 & 1144 & 1297 & 1450 & 1460 & $16 \% 2$ & 1728 & 1728 & 168 & 198 & 214 & 214 & 243 & 271 & 271 & 100 & 323 & 323 \\
\hline ION 112 & 710 & $\pi 3$ & 871 & 932 & 995 & 1115 & 1174 & 1232 & 1473 & 1473 & 324 & 353 & 390 & 426 & 465 & 510 & 537 & & 73 & 673 \\
\hline moN 1:2 & 826 & 869 & 988 & 1068 & 1116 & 1247 & 1310 & 1372 & 1478 & 1538 & 340 & 376 & $41 B$ & 440 & 472 & 527 & 557 & 96 & 25 & 658 \\
\hline RAIDiNO0D 1 & 969 & 1117 & 1117 & 1244 & 1300 & 1390 & 1486 & 1508 & 1508 & 1701 & 418 & 472 & 472 & 528 & 575 & 575 & 829 & 376 & 76 & 720 \\
\hline Io ROCX 1 & 115 & 115 & 115 & 115 & 115 & 115 & 115 & 115 & 116 & 116 & 15 & 15 & 15 & 16 & 15 & 15 & 15 & 15 & 15 & 15 \\
\hline ITNE YANKE & & 381 & 411 & 470 & 470 & 536 & & 5 & 530 & 630 & 137 & 137 & 158 & 179 & 179 & 282 & 292 & 12 & 02 & 292 \\
\hline ORT CNHOW & 246 & 246 & 275 & 311 & 311 & 347 & 381 & 381 & 381 & 381 & 89 & 80 & 9 & 112 & 112 & 125 & 137 & 37 & 37 & 137 \\
\hline AYIS-8ESSE 1 & 398 & 339 & 379 & 420 & 429 & 479 & 520 & 529 & 570 & 612 & 168 & 158 & 177 & 281 & 261 & 224 & 248 & 248 & 267 & 286 \\
\hline CROSSE & 91 & 9 & 100 & 106 & 180 & 168 & 168 & 106 & 186 & 168 & 15 & 15 & 17 & 17 & 17 & 17 & 17 & 17 & 17 & 17 \\
\hline ARICO FERUI & 1040 & 1208 & 1296 & $\$ 545$ & 1797 & 1707 & 2655 & 2035 & 2232 & 2232 & 190 & 238 & 236 & 261 & 327 & 327 & 370 & $\theta$ & 96 & 486 \\
\hline AN ONGFRE 1,2 & 883 & 883 & 5840 & 1271 & 1349 & 1424 & 1497 & 1571 & 1633 & 1690 & 398 & 386 & 431 & 521 & 564 & 586 & 617 & 649 & 76 & 702 \\
\hline NALE CTY $1 \mathrm{~A}$ & & 2998 & 2237 & 2422 & 2894 & 2006 & 3164 & 3528 & & 3830 & 11 & 380 & & 441 & & 43 & 78 & 42 & 89 & 697 \\
\hline RESDEN 2 & 671 & 868 & 898 & 943 & 843 & 943 & 243 & 943 & 943 & 943 & 112 & 135 & 135 & 158 & 156 & 158 & 158 & & 58 & 158 \\
\hline ESDEN 3 & 477 & 614 & 721 & 721 & 858 & 989 & 980 & 989 & 980 & 989 & 8 & 103 & 121 & 121 & 144 & 168 & 156 & 36 & 166 & 166 \\
\hline MAD CITIES & 1188 & 1446 & 1554 & 1691 & 1889 & 2103 & 2236 & 2601 & 3213 & 3213 & 200 & 255 & & 98 & & 1 & 94 & & 38 & 566 \\
\hline ADOAU NECK & 231 & 277 & $2 \pi$ & 27 & $2 \pi$ & $2 \pi$ & 277 & $2 \pi$ & $2 \pi$ & $2 \pi$ & 95 & 114 & 114 & 114 & 114 & 114 & 114 & 4 & 14 & 114 \\
\hline LUCIE 2 & 246 & 300 & 357 & 357 & 420 & 480 & 4810 & 639 & 588 & 580 & 90 & 120 & 139 & 139 & 103 & 187 & 187 & 8 & 229 & 229 \\
\hline RKEY PY 1L2 & 360 & 388 & 431 & 501 & 542 & 580 & 658 & 945 & a45 & 845 & 163 & 183 & 198 & 230 & 249 & 266 & 298 & & 98 & 388 \\
\hline LUCIE 1 & 368 & 388 & 359 & 420 & 421 & 482 & 543 & 543 & 592 & 045 & 118 & 110 & 137 & 160 & 160 & 184 & 267 & 77 & 28 & 246 \\
\hline $10 \times 112$ & 866 & 882 & 797 & 864 & 941 & 1007 & 1079 & 1217 & 1271 & 1524 & 283 & 294 & 344 & 374 & 405 & 438 & 485 & & 49 & 682 \\
\hline TZPATRICK & 742 & 741 & 863 & 1809 & 1060 & 1156 & 1297 & 1297 & 1417 & 1539 & 132 & 132 & 153 & 179 & 178 & 295 & 230 & & 252 & 273 \\
\hline AANE ARNOLD & 423 & 423 & 505 & 604 & 684 & 704 & 603 & 808 & 886 & 972 & 75 & 75 & 90 & 190 & 168 & 125 & 143 & & 58 & 173 \\
\hline SH MULEKR 2 & 9103 & 1042 & 1151 & 1286 & 1427 & 1589 & 1691 & 1822 & 1931 & 2044 & 159 & 183 & 203 & 228 & 251 & 275 & 298 & & 10 & 360 \\
\hline K MCLEAR 2 & 127 & 198 & 233 & 233 & 292 & 282 & 347 & 404 & 484 & 449 & $\mathbf{5 3}$ & 77 & 97 & 97 & 121 & 121 & 144 & & 68 & 186 \\
\hline K NUEEAR 1 & 125 & 175 & 175 & 222 & 222 & 269 & 316 & 316 & 355 & 365 & 58 & 91 & 81 & 103 & 103 & 125 & 146 & & 65 & 185 \\
\hline$R A Y 112$ & 1567 & 1713 & 1878 & 2337 & 2488 & 2736 & 3160 & 3297 & 3568 & 3658 & 285 & 312 & 342 & 425 & 454 & 497 & 575 & & 38 & 685 \\
\hline JNNEE & 108 & 291 & 228 & 256 & 288 & 319 & $35:$ & 379 & 404 & 494 & 64 & 76 & $B 8$ & 97 & 169 & 121 & 133 & 144 & 153 & 153 \\
\hline LEV 1 & 179 & 253 & 310 & 316 & 384 & 454 & 484 & $\mathbf{5 2 3}$ & 581 & 591 & & 118 & 142 & 142 & 178 & 298 & 288 & & 167 & 267 \\
\hline LES UNIT 2 & 220 & 293 & 293 & 384 & 438 & 436 & 586 & 575 & 575 & 633 & & 135 & 135 & 187 & 291 & 201 & 232 & & 66 & 292 \\
\hline INTOK 1 & 379 & 502 & $5 \oplus 2$ & 677 & 815 & 815 & 967 & 1880 & 1988 & 1218 & 69 & 92 & 92 & 124 & 149 & 149 & 177 & 198 & 198 & 222 \\
\hline INNA & 103 & 131 & 153 & 179 & 297 & 287 & 207 & 287 & 207 & 207 & 34 & 43 & 50 & 59 & 88 & 89 & 68 & 68 & 68 & 68 \\
\hline
\end{tabular}


TABLE A.8. Upper Reference Case, Maximum At-Reactor Capacity Plus

Transshipment--Projected Cumulative Storage Requirements

(contd)

ASSERIES

POOL

RACHO SEED-1

VT YNAKE 1

HATCH 112

SUSPUENAM 112

B VALEY 2

8 VNUEY 1

gROTLS FERTYL

BROWIS FERTY3

PALO VEDE 2

PALO VEREE :

PNO YEROE 3

CRTSTAL RVR 3

VOCRE IIS

TRDJAN

INOIAN PT 3

SERUOTAH 112

TATTS QAR 142

SELFONTE 1

BELEONTE 2

CALAIAY 1

HARRIS 1

ROEIXSON 2

RNR BEN 1

WATEFORO 3

HENTICE丩O

FADEY 2

FARLY 1

MILSTRNE 2

UILLSTONE 3

OIAR O CAYYON-1

DIAEL CAYON-2

NIME UILE PT2

WCUIRE 1

OCONE 110

DCONEX 3

WCUIRE 2

CATAMEA 1

CATATEA 2

HOPE CREX

WOLF CREEX 1

SUMER 1

COMACHE PK 112 SHOREWH

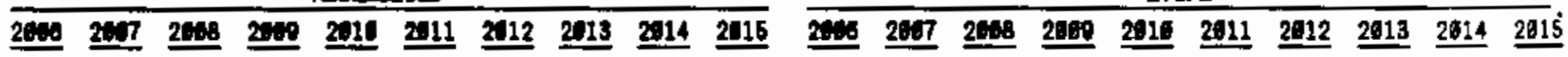

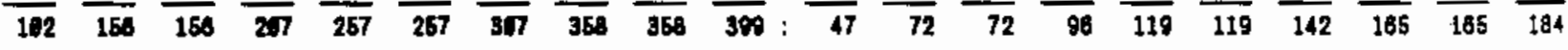

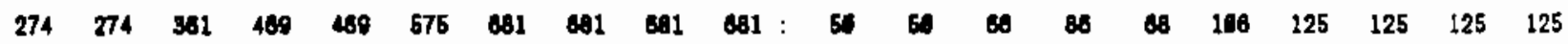

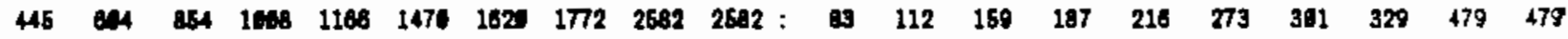
$\begin{array}{lllllllllllllllllllll}539 & 734 & 1442 & 1233 & 1428 & 1844 & 1986 & 2171 & 2477 & 2695 & : & 93 & 127 & 189 & 213 & 247 & 311 & 343 & 375 & 428 & 455\end{array}$

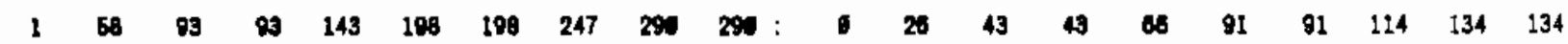
$\begin{array}{llllllllllllllllllll}63 & 0 & 112 & 173 & 237 & 237 & 295 & 355 & 355 & 477 & 2 & 29 & 52 & 80 & 109 & 109 & 136 & 184 & 164 & 188\end{array}$ $\begin{array}{lllllllllllllllllllll}316 & 316 & 627 & 1016 & 1610 & 1389 & 1389 & 1785 & 277 & 2841 & : & 57 & 57 & 114 & 183 & 183 & 252 & 252 & 328 & 377 & 516\end{array}$

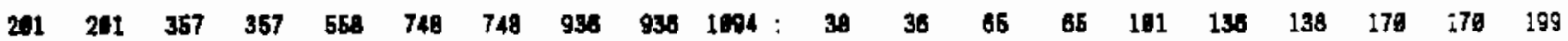

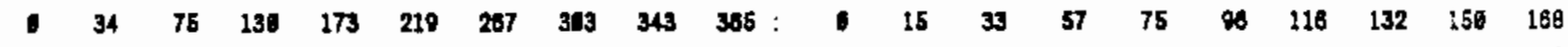

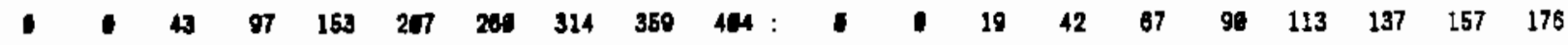

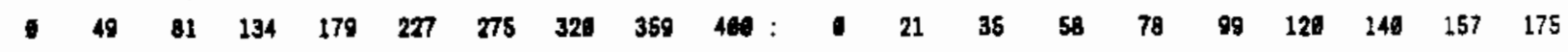

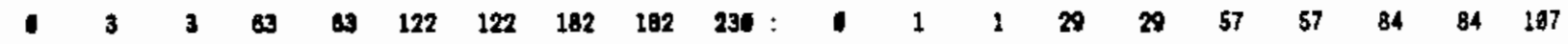

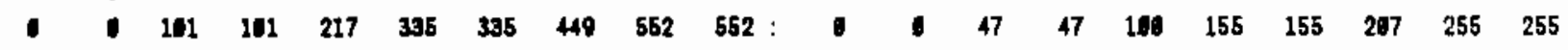

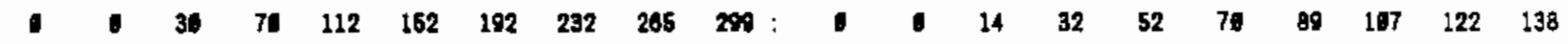

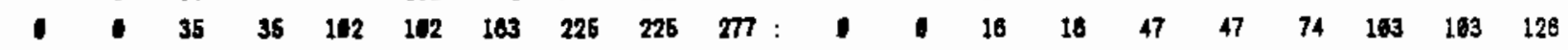

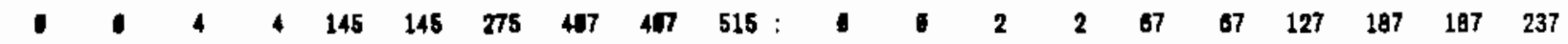

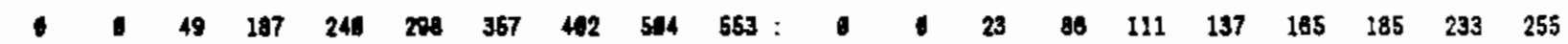

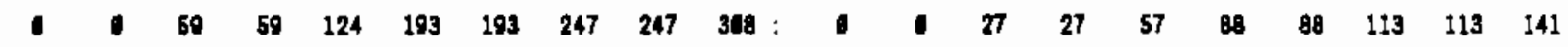

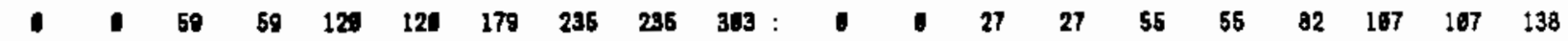

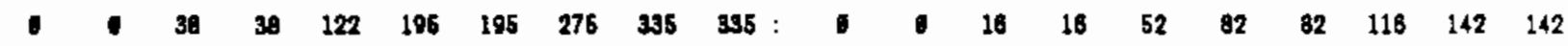

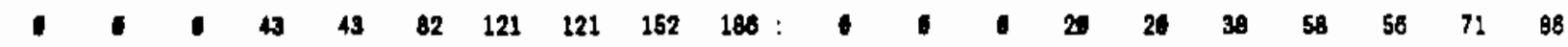

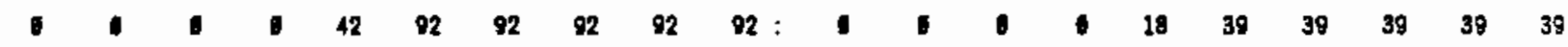

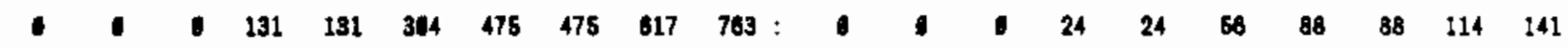

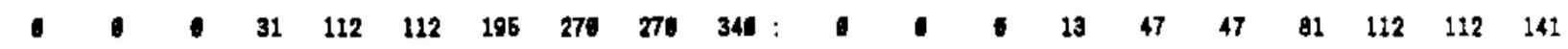

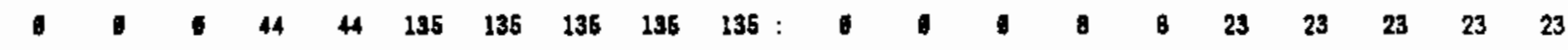

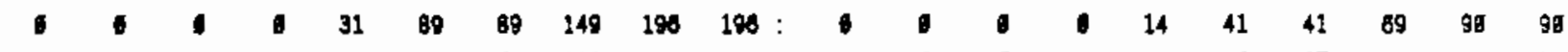

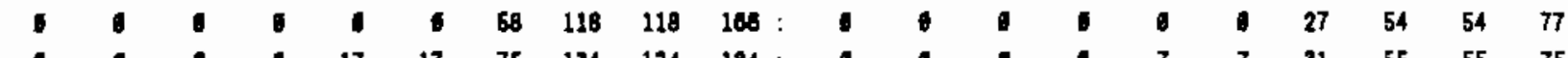

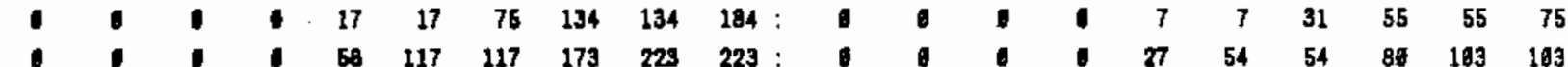

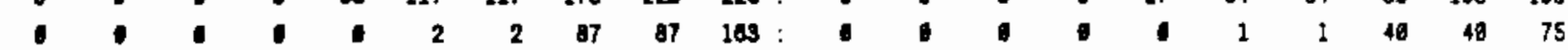

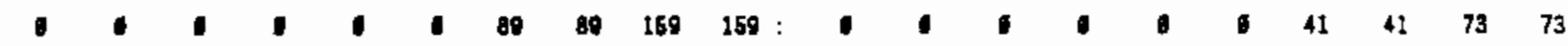

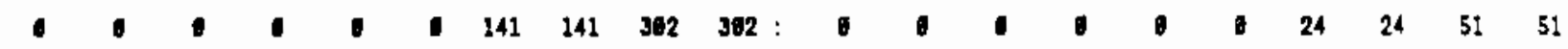

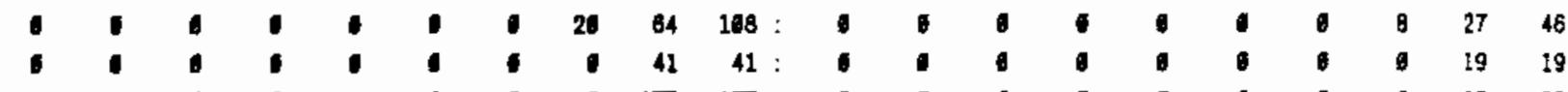

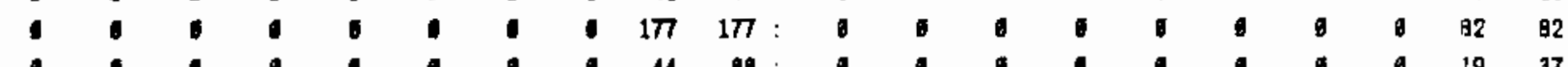

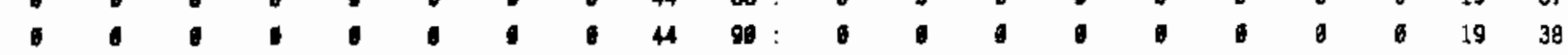

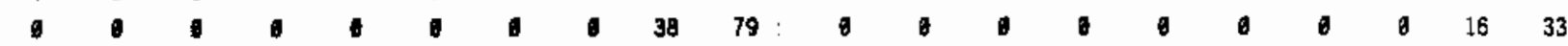

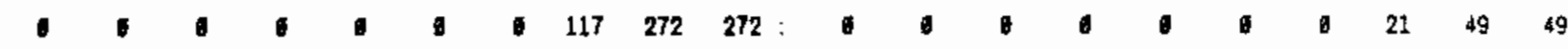

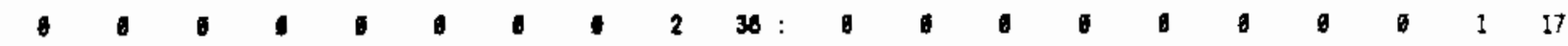

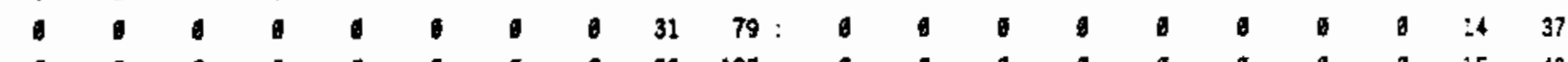

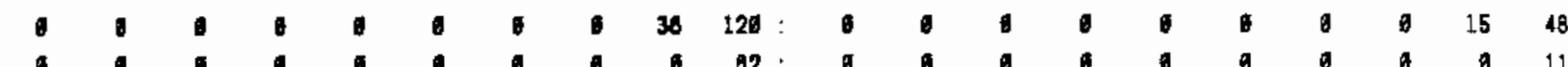


TABLE A.8. Upper Reference Case, Maximum At-Reactor Capacity Plus Transshipment--Projected Cumulative Storage Requirements (contd)

A5SEgalES

ITIHI

POOL. 2006 2017 2016 2000 2011 2011 2012 2013 2014 2015

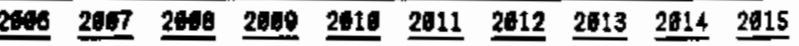

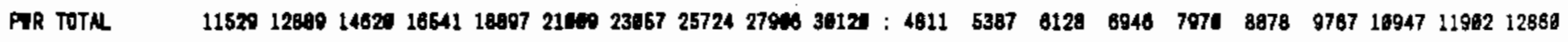

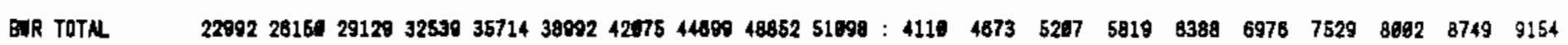

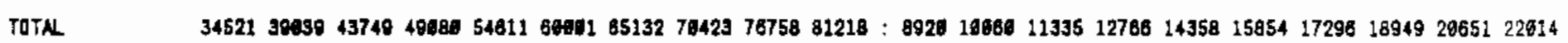


TABLE A.8. Upper Reference Case, Maximum At-Reactor Capacity Plus Transshipment--Projected Cumulative Storage Requi rements (contd)

\begin{tabular}{|c|c|c|c|c|c|c|c|c|c|c|}
\hline \multirow[b]{2}{*}{ POOL } & \multicolumn{5}{|c|}{ ASSE':IES } & \multicolumn{5}{|c|}{ जTIH } \\
\hline & 2018 & $\underline{2017}$ & $\underline{2018}$ & $\underline{2010}$ & $\underline{2120}$ & $\underline{2016}$ & $\underline{2017}$ & $\underline{2018}$ & 2019 & 2620 \\
\hline PALISADES & 238 & 238 & $\overline{238}$ & 238 & 238 & 93 & 93 & $\overline{93}$ & 93 & 93 \\
\hline MILSTINE 1 & 2232 & 2232 & 2232 & 2232 & 2232 & 397 & 397 & 397 & 397 & 397 \\
\hline PILCRIV 1 & 1461 & 1461 & 1461 & 1461 & $i 461$ & 258 & 258 & 258 & 258 & 258 \\
\hline CALYER CF IL2 & 1851 & 1923 & 1923 & 1923 & 1923 & 807 & 724 & 724 & 724 & 724 \\
\hline OYSTER CRK 1 & 1213 & 1213 & 1213 & 1213 & 1213 & 214 & 214 & 214 & 214 & 214 \\
\hline PRAIRIE ISL $1 \perp 2$ & 1280 & 1289 & I289 & 1289 & 1289 & 457 & 457 & 457 & 457 & 457 \\
\hline NORTH ANM IL & 1495 & 1591 & 1747 & 1700 & 1837 & 891 & 735 & 897 & 825 & 849 \\
\hline SLART 142 & 1173 & 1173 & $117 \mathrm{~s}$ & 1173 & 1173 & 539 & 538 & 538 & 538 & 538 \\
\hline IHDTAN PT 2 & 808 & 898 & 809 & 688 & 508 & $\pi 74$ & 274 & $\pi 4$ & 274 & 274 \\
\hline COOPER STN & 1874 & 1874 & 1674 & 1874 & 1674 & 395 & 305 & 305 & 385 & 305 \\
\hline LIMERICX I & 2380 & 2549 & 2549 & 2689 & 2881 & 422 & 452 & 452 & 477 & 507 \\
\hline PEACHBQTTOU 2 & 2173 & 2173 & 2173 & 2173 & 2173 & 385 & 385 & 395 & 385 & 385 \\
\hline PEACHEATTON 3 & 2334 & 2330 & 2330 & 2339 & 2330 & 413 & 413 & 413 & 413 & 413 \\
\hline LIHERICK 2 & 2678 & 2876 & 2878 & 3055 & 3276 & 478 & 513 & 513 & 545 & 584 \\
\hline POIMT DEACH IL2 & 293 & 933 & 933 & 233 & 933 & 331 & $33:$ & 331 & 331 & 331 \\
\hline Bauss ICK 1 & 1150 & 1150 & 1158 & 1159 & 1159 & 217 & 217 & 237 & 217 & 217 \\
\hline BRALSTICX 2 & 1728 & 1728 & 1728 & 1728 & 1728 & 323 & 323 & 323 & 323 & 323 \\
\hline ZION 112 & 1473 & 1473 & 1473 & 1473 & 1473 & 673 & 873 & 673 & 873 & 673 \\
\hline EXRON 112 & 1588 & 1712 & 1783 & 1827 & 1953 & 672 & 724 & 754 & $\pi 3$ & 828 \\
\hline genIOT000 112 & 1805 & 1895 & 1929 & 2418 & 2016 & 784 & 784 & 818 & 863 & 853 \\
\hline BIO ROCK 1 & 115 & 115 & 115 & 115 & 145 & 15 & 15 & 15 & 15 & 15 \\
\hline WINE YANKEE & 536 & 530 & 530 & 530 & 530 & 242 & 202 & 262 & 202 & 262 \\
\hline FORT CNHOUN & 381 & 391 & 381 & 381 & 391 & 137 & 137 & 137 & 137 & 137 \\
\hline DAYIS-BESSE 1 & 812 & 650 & 716 & 710 & 710 & 286 & 388 & 332 & 332 & 332 \\
\hline LACROSSE & 100 & 186 & 106 & 168 & 119 & 17 & 17 & 17 & 17 & 17 \\
\hline ENRICO FERII-2 & 2444 & 2673 & 2673 & 2863 & 2803 & 445 & 488 & 486 & 521 & 521 \\
\hline SAN ONOFRE $1,2,13$ & 1764 & 1902 & 1902 & 2617 & $2 \varsigma 17$ & 739 & 790 & 796 & 848 & 840 \\
\hline LNSWE CTY IL & 4145 & 4318 & 4584 & 4787 & 4981 & 754 & 788 & 826 & 871 & 903 \\
\hline ORESOEN 2 & 943 & 943 & 943 & 943 & 943 & 158 & 158 & 158 & 158 & 158 \\
\hline DRESOEN 3 & 989 & 989 & 989 & 989 & 989 & 108 & 160 & 1BB & 186 & 186 \\
\hline QUD CITIES $1 \$ 2$ & 3213 & 3213 & 3213 & 3213 & 3213 & 586 & 586 & 589 & 568 & 588 \\
\hline HACON MECX & $2 n$ & 27 & $2 \pi$ & 277 & $2 \pi$ & 114 & 114 & 114 & 114 & 114 \\
\hline ST LUCIE 2 & 639 & 695 & 895 & 741 & 798 & 240 & 270 & 276 & 288 & 310 \\
\hline TURKEY PT 112 & 845 & 845 & 845 & 845 & 845 & 388 & 388 & 388 & 388 & 386 \\
\hline ST LUCIE 1 & 645 & 645 & 645 & 645 & 845 & 248 & 248 & 248 & 246 & 248 \\
\hline COOK 112 & 1624 & 1593 & 1887 & 1687 & 1887 & 882 & 894 & 729 & 729 & 729 \\
\hline FITZPATRICX & 1539 & 1539 & 1539 & 1539 & 1539 & 273 & 273 & 273 & 273 & 273 \\
\hline DUANE ARHDLD & 972 & 972 & 972 & 972 & 972 & 173 & 173 & 173 & 173 & 173 \\
\hline WASH MUCLEAR 2 & 2158 & 2284 & 2419 & 2522 & 2650 & 389 & 402 & $42 B$ & 444 & 487 \\
\hline ARK MUCEAR 2 & 448 & 504 & 581 & 581 & 316 & 188 & 289 & 233 & 239 & 258 \\
\hline ARK MUCLEAR 1 & 355 & 355 & 355 & 355 & 355 & 165 & 185 & 185 & 185 & 165 \\
\hline PERAY 112 & 3878 & 4272 & 4458 & 4881 & 5070 & 705 & 777 & 811 & 846 & 922 \\
\hline KEAUNEE & 404 & 484 & 494 & 464 & 404 & 153 & 153 & 153 & 153 & 153 \\
\hline SNEI ! & 841 & 707 & 707 & 707 & 707 & 294 & 325 & 325 & 325 & 325 \\
\hline SALE UNIT 2 & 893 & 893 & 784 & 819 & 819 & 320 & 326 & 352 & 378 & 378 \\
\hline GINTON 1 & 1358 & 1358 & 1517 & 1853 & 1653 & 248 & 248 & 277 & 392 & 362 \\
\hline GINNA & 207 & 297 & 207 & 297 & 267 & 88 & 68 & 88 & 68 & $B B$ \\
\hline RANCHO SECD-I & 399 & 399 & 399 & 399 & 399 & 184 & 184 & 184 & 184 & 184 \\
\hline
\end{tabular}


TABLE A.8. Upper Reference Case, Maximum At-Reactor Capacity Plus Transshipment--Projected Cumulative Storage Requi rements (contd)

\begin{tabular}{|c|c|c|c|c|c|c|c|c|c|c|}
\hline \multirow[b]{2}{*}{ PODL. } & \multicolumn{5}{|c|}{ ASSERIES } & \multicolumn{5}{|c|}{ YTIH } \\
\hline & 2016 & 2117 & 2918 & 2019 & $\underline{2021}$ & $\underline{2618}$ & 2017 & $2: 18$ & 2019 & 2.12 \\
\hline VT YANEEE I & $\mathbf{\infty 1}$ & 81 & 891 & 681 & 681 & 125 & 125 & 125 & 125 & 125 \\
\hline maTCH 112 & 2714 & 2859 & 2858 & 2979 & 2979 & BA & 551 & 631 & 553 & 553 \\
\hline SUSAENNNA 142 & 2706 & 3150 & 3940 & 3488 & 3847 & 493 & 544 & 577 & 662 & 885 \\
\hline B YNEY 2 & $\mathbf{3 3 7}$ & 388 & 388 & 428 & 478 & 155 & 179 & 170 & 108 & 224 \\
\hline 9 VแEY 1 & 460 & 489 & 481 & 480 & 460 & 212 & 212 & 212 & 212 & 212 \\
\hline BRonis FERTIL2 & 2841 & 2841 & 2841 & 2841 & 2841 & 510 & 516 & 516 & 518 & 518 \\
\hline BRDTS FERRT3 & 1258 & 1268 & 1258 & 1268 & 1250 & 228 & 228 & 228 & 228 & 228 \\
\hline PNO VEDE 2 & 420 & 478 & 513 & 554 & 590 & 187 & $2 \mathrm{CB}$ & 224 & 242 & 201 \\
\hline PAL VEOE 1 & 451 & 542 & 658 & 597 & 248 & 197 & 219 & 243 & 260 & 283 \\
\hline PNL VERDE 3 & 446 & 485 & 533 & 588 & 813 & 192 & 212 & 293 & 248 & 267 \\
\hline CRISTA RYR 3 & 231 & 285 & 285 & 205 & 285 & 107 & 132 & 132 & 132 & 132 \\
\hline VOGTLE IL2 & 657 & 792 & 782 & 871 & 988 & 303 & 301 & 361 & 482 & 458 \\
\hline TRUJNY & 334 & 334 & 334 & 334 & 334 & 154 & 154 & 154 & 164 & 154 \\
\hline IMDIAN PT 3 & 27 & 27 & 277 & 277 & 277 & 128 & 128 & 128 & 228 & 128 \\
\hline SERUOY새 $1 / 2$ & 572 & 634 & 768 & 760 & 894 & 263 & 292 & 353 & 353 & 411 \\
\hline WATTS RAR 142 & 648 & 655 & $7 \$ 1$ & 803 & 649 & 286 & 302 & 323 & 371 & 392 \\
\hline BELFONTE 1 & 373 & 373 & 445 & 568 & 390 & 170 & 170 & 203 & 232 & 232 \\
\hline BבLEONTE 2 & 367 & 367 & 425 & 497 & 407 & 183 & 163 & 194 & 222 & 222 \\
\hline CNUANAY 1 & 402 & 471 & 471 & 531 & $6: 12$ & 170 & 180 & 190 & 225 & 255 \\
\hline MWRIS 1 & 186 & 222 & 252 & 262 & 289 & 80 & 183 & 117 & 117 & 134 \\
\hline ROEIISON 2 & 92 & 92 & 92 & 92 & 92 & 39 & 39 & 39 & 30 & 39 \\
\hline ANR GOD 1 & 783 & 928 & 1104 & 1184 & 1271 & 141 & 171 & 204 & 204 & 235 \\
\hline VATEFFORO 3 & 405 & 415 & 491 & 550 & 558 & 168 & 168 & 204 & 229 & 229 \\
\hline 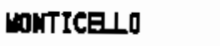 & 136 & 135 & 136 & 135 & 135 & 23 & 23 & 23 & 23 & 23 \\
\hline FAREY 2 & 248 & 392 & $3: 12$ & 340 & 413 & 114 & 139 & 139 & 101 & 188 \\
\hline FAREY 1 & 218 & 218 & 218 & 218 & 218 & 191 & 181 & 181 & 161 & 191 \\
\hline MILSTONE 2 & 184 & 184 & 184 & 184 & 184 & 75 & 75 & 75 & 75 & 75 \\
\hline MนSTUNE 3 & 275 & 337 & 397 & 384 & 438 & 127 & 155 & 165 & 175 & 262 \\
\hline DINBO CArYON-1 & 103 & 244 & 244 & 315 & 315 & 75 & 112 & 112 & 145 & 145 \\
\hline DIAQD CAMYON-2 & 238 & 236 & 325 & 325 & 412 & 100 & 108 & 149 & 149 & 189 \\
\hline NINE NILE PT2 & 471 & 471 & 670 & 879 & 872 & 86 & B9 & 218 & 118 & 149 \\
\hline MCEIPE 1 & 188 & 158 & 211 & 252 & 252 & 46 & 67 & 89 & 107 & 107 \\
\hline oconze 142 & 41 & 41 & 41 & 41 & 41 & 19 & 19 & 19 & 19 & 19 \\
\hline OCONEE 3 & 177 & 17 & 177 & 17 & 177 & 82 & 82 & 82 & 82 & 82 \\
\hline LEEIRE 2 & 134 & 184 & 164 & 225 & 276 & 57 & 78 & 78 & 95 & 117 \\
\hline CATATEA 1 & 197 & 197 & 192 & 293 & 284 & 58 & 58 & 81 & 99 & 120 \\
\hline CATANBA 2 & 119 & 187 & 187 & 201 & 246 & 39 & 71 & 71 & 85 & 104 \\
\hline HOPE CREA & 438 & 819 & 619 & 758 & 945 & 79 & 113 & 113 & 138 & 172 \\
\hline WOLF CREEX 1 & 76 & 168 & 148 & 170 & 217 & 32 & 50 & 89 & 83 & 101 \\
\hline SUMG : & 79 & 132 & 108 & 188 & 242 & 37 & 61 & 87 & 87 & 112 \\
\hline COUAKCHE PK 122 & 293 & 298 & 387 & 463 & 568 & 82 & 128 & 158 & 187 & 224 \\
\hline SHOREMA & 82 & 168 & 382 & $3 \oplus 2$ & 494 & 11 & 31 & 55 & 55 & 79 \\
\hline TNP-1 & 78 & 78 & 163 & 243 & 243 & 38 & 38 & 74 & 111 & 111 \\
\hline WNP-3 & 59 & 119 & 17 & 231 & 300 & 28 & 52 & 77 & 181 & 131 \\
\hline SEABOOKK 2 & 6 & 0 & g & a & 17 & 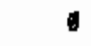 & 6 & 0 & 1 & B \\
\hline
\end{tabular}


TABLE A.8. Upper Reference Case, Maximum At-Reactor Capacity Plus Transshipment--Projected Cumulative Storage Requirements (contd)

POQL

NS5ERIES

ITHH

2016 2017 2018 2619 2920 2016 2017 2018 2019 2026

PIR TOTAL

$3195133859355813790538594: 1387114517152581503318594$

BRT TOTN

$5344355286506515838069444: 96949097181631044816831$

TOTAL

8499499144922229539599138 : 23175 24414264112638127425 
APPENDIX B

MAP OF FEDERAL ENERGY REGIONS 


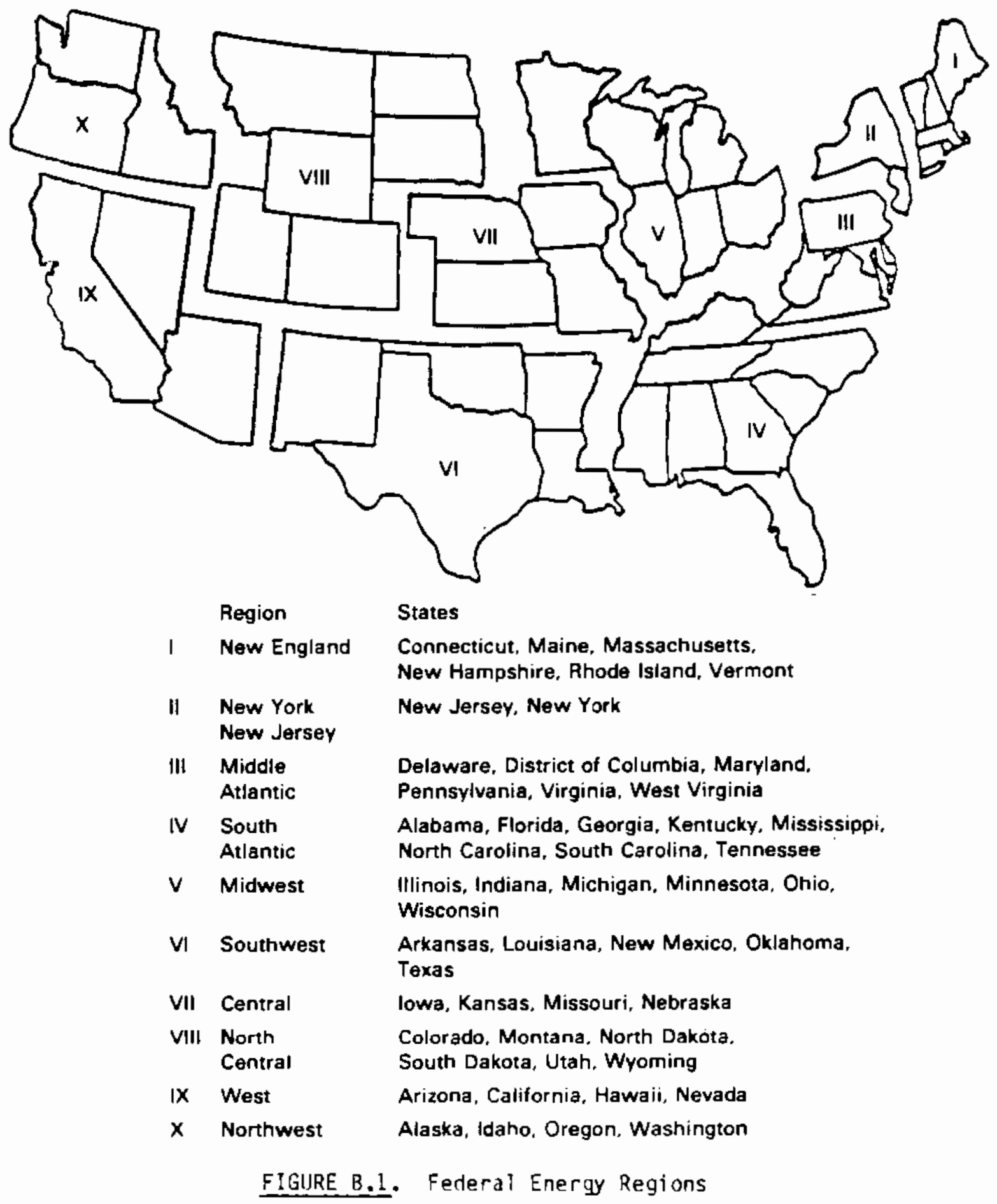


. 


\section{DISTRIBUTION}

No. of

Copies

OFFSITE

L. H. Barrett

$\mathrm{RW}-33$

Office of Civilian Radiaactive Waste Management

U.S. Department of Energy

Washington, DC 20585

J. H. Carlson

$\mathrm{RW}=32$

Office of Civilian Radioactive Waste Management

U.S. Department of Energy

Washington, DC 20585

R. G. Clark

Director of Nuclear and Alternate Fuels (EI-53)

U.S. Department of Energy

Washington, DC 20585

C. W. Conner

$\mathrm{RW}-12$

Office of Civilian Radioactive Waste Management

U.S. Department of Energy

Washington, DC 20585

\section{J. Disbrow}

EI-53]

Energy Information

Administration

U.S. Department of Energy

Washington, DC 20585

J. Epstein

$\mathrm{RW}-32$

Office of Civilian Radioactive Waste Management

U.S. Department of Energy

Washington, DC 20585
No. of

Copies

F. P. Falci

$0 P-121$

U.S. Department of Energy

Washington, DC 20545

J. S. Finucane

EI-531

Energy Information

Administration

U.S. Department of Energy

Washington, DC 20585

W. A. Frankhauser

DP-122.1

U.S. Department of Energy

Washington, DC 20545

H. J. Hale

RW- 33

Office of Civilian Radioactive Waste Management

$U$. S. Department of Energy

Washington, DC 20585

U. R. Hilley

$R W-30$

Office of Civilian Radioactive Waste Management

U.S. Department of Energy

Washington, DC 20585

T. H. Isaacs

RW-20

Office of Civilian Radioactive Waste Management

U.S. Department of Energy

Washington, DC 20585

K. A. Klein

$\mathrm{RW}-32$

Office of Civilian Radioactive Waste Management

U.S. Department of Energy

Washington, DC 20585 
No. of

Copies

E. F. Mastel

NE -72

U.S. Department of Energy

Washington, OC 20545

20

R. A. Milner

RW-12

Office of Civilian Radioactive Waste Management

U.S. Department of Energy

Washington, OC 20585

T. D. Nguyen

RW-33

Office of Civilian Radioactive Waste Management

U.S. Department of Energy

Washington, DC 20585

M. L. Payton

RW-12

Office of Civilian Radioactive Waste Management

U.S. Oepartment of Energy

Washington, DC 20585

R. Philpott

$R W-33$

Office of Civilian Radioactive Waste Management

U.S. Department of Energy

Washington, DC 20585

D. E. Shelor

RW-32

Office of Civilian Radioactive Waste Management

U.S. Department of Energy

Washington, DC 20585

H. Steinberg

RW-33

Office of Civilian Radioactive Waste Management Information U.S. Department of Energy Washington, OC 20585
No. of

Copies

\author{
W. M. Sprecher \\ RW-42 \\ Office of Civilian Radioactive \\ Waste Management \\ U.S. Department of Energy \\ Washingt on, DC 20585 \\ E. L. Wilmot \\ RW-33 \\ Office of Civilian Radioactive \\ Waste Management \\ U.S. Department of Energy \\ Washington, DC 20585 \\ R. C. Ashline \\ Oak Ridge National Laboratory \\ P.0. Box $X$ \\ Oak Ridge, TN 37930 \\ J.H. Saling \\ Oak Ridge National Laboratory \\ P.0. Box $X$ \\ 0ak Ridge, TN 37830 \\ J. A. Klein \\ Oak Ridge National Laboratory \\ P.O. Box $X$ \\ Oak Ridge, TN 37830 \\ D. S. Joy \\ Oak Ridge National Laboratory \\ P.0. Box $X$ \\ Oak Ridge, iN 37830 \\ S. N. Storch \\ Oak Ridge National Laboratory \\ P.0. Box $X$ \\ Oak Ridge, TN 37830 \\ X. Goltiher \\ DOE Albuquerque Operations \\ Office \\ P.0. $80 \times 5400$ \\ Albuquerque, NM 87115
}


No. of

Copies

G. C. Allen

Sandia National Laboratories P.0. Box 5800

Albuquerque, NM 87185

J. W. Cashwell

Sandia National Laboratories

P.0. Box 5800

Albuquerque, NM 87185

2 E. R. Johnson

JAI

11702 Bowman Green

Reston, VA 22090

R. Piscitella

EG\&G Idaho

P.0. Box 1625

Idaho Falls, ID 83415

R. W. Lambert

Electric Power Research Institute

3412 Hillview Avenue

Palo Alto, CA 94303

R. F. Williams

Electric Power Research Institute

3412 Hillview Avenue

Palo Alto, CA 94303

8. Agarwal

FW Energy Applications

20 North Clark St.

Chicago, IL 606

R. P. Morissette

Science Applications Inc.

Valley Bank Center

101 Convention Center Orive

Suite 407

Las Vegas, Nevada 89109

B. F. Judson

General Electric Company

175 Cortner Avenue

San Jose, CA 95630
No. of

Copies

Young Park

NUS Corporation

910 Clopper Road

Gaithersburg, MD 20878

B. R. Teer

Transnuclear, Inc.

One North Broadway

White Plains, NY 10601

Edward Kuhn

Stone Webster Engineering Corp.

One Penn Plaza

250 West 34th Street

New York, NY 10119

M. Conroy

Roy $F$. Weston, Inc.

955 L'Enfant Plaza S.W.

Eighth Floor

washington, DC 20585

J. Lilly

Roy $F$. Weston, Inc.

955 L'Enfant P1aza S.W.

Eighth Floor

Washington, DC 20585

A. Leiter

Roy F. Weston, Inc.

955 L'Enfant Plaza \$.W.

Eighth Floor

Washington, OC 20585

F. Haines

Roy $F$. Weston, Inc.

955 L'Enfant Plaza S.W.

Eighth Floor

Washington, DC 20585 
No, of

Copies

M. Rahimi

Roy $F$. Weston, Inc.

955 L'Enfant PTaza S.W.

Eighth Floor

Washington, DC 20585

R. R. McDonald

Roy $F$. Weston, Inc.

1 Weston Way

Westchester, PA 19380

30 DOE Technical Information

Center

ONSITE

4 DOE Richland Operations Office

D. E. Crouter

J. M. Peterson (2)

0 . C. Langstaff

70 Pacific Northwest Laboratory

M. K. Altenhofen

W. J. Bailey

G. H. Beeman

A. J. Boegel

J. L. Braitman

L. L. Clark

B. M. Cole

C. A. Counts

J. M. Creer

P. M. Daling
No. of

Copies

L. R. Dodd

A. L. Doherty

K. A. Exblaw

R. L. Engel

J. F. Fletcher

E. R. Gilbert

C. M. Heeb (10)

G. M. Holter

A. B. Johnson

G. J. Konzek

R. A. Libby

W. W. Little

A. T. Luksic

C. A. Mackay (5)

J. L. McEl roy

R. W. McKee

G. W. McNair

D. F. Newman

W. L. Purcell

R. G. Rau

F. P. Roberts

A. F. Rosenfeld

N. L. Sevigny

M. R. Shay

R. I. Smith

L. A. Strope

M. B. Triplett

R. C. Walling (10)

M. K. White

$R$. S. Wegeng

T. W. Wood

Publishing Coordination MH (2)

Technical Information (5) 